\title{
The Tumor Microenvironment in Preclinical Models of Brain Metastasis with a Focus on Tumor-associated Macrophages and Microglia and Effects of Whole-Brain Radiotherapy
}

\author{
Dissertation \\ zur Erlangung des Doktorgrades \\ der Naturwissenschaften
}

\begin{abstract}
vorgelegt beim
Fachbereich Biowissenschaften (15)

der Johann Wolfgang Goethe-Universität

in Frankfurt am Main
\end{abstract}

\author{
von \\ Michael Schulz \\ aus Dresden
}

Frankfurt 2021

D30 
vom Fachbereich für Biowissenschaften (FB 15) der

Johann Wolfgang Goethe - Universität als Dissertation angenommen.

\section{Dekan}

Prof. Dr. Sven Klimpel

\section{Gutachter}

Prof. Dr. Amparo Acker-Palmer (Hauptgutachterin)

PD Dr. Patrick N. Harter (Zweitgutachter)

\section{Datum der Disputation}

09.09.2021 
This doctoral thesis was performed and prepared in the group of Dr. Lisa Sevenich at the Georg-Speyer-Haus (Frankfurt) from November 2015 until March 2021.

Parts of this thesis have been published (p. 148). 
"The good thing about science is that it's true whether or not you believe in it"

Neil deGrasse Tyson 


\section{Table of contents}

$\begin{array}{lr}\text { I Abbreviations } & \text { p. } 10\end{array}$

$\begin{array}{lr}\text { Figures and Tables } & \text { p. } 12\end{array}$

II Zusammenfassung/Summary p. 14

Graphical overview............................................... p. 14

A) Zusammenfassung - deutsche Version......................... p. 15

B) Summary - english version .............................. p. 21

$\begin{array}{lr}\text { III Introduction and state of the art } & \text { p. } 27\end{array}$

A) Brain metastasis.......................................... p. 27

B) Induction of brain metastasis.............................. p. 27

C) Brain metastasis microenvironment - brain-resident cells .... p. 28

D) Brain metastasis microenvironment - recruited cells........... p. 31

E) Brain metastasis: diagnosis and treatment................. p. 34

$\begin{array}{lr}\text { IV Aims and questions } & \text { p. } 38\end{array}$

V Material and Methods p. 39

Simplified scheme - Overview of thesis methodology.................. p. 39

Resource identifier - material and devices........................... 40

A) Cell lines and primary cells............................... p. 43

1. Tumor cell lines....................................p. 43

a) Maintenance and seeding for generation

of supernatant....................................... 43

b) Viral transduction................................. p. 44

2. Microglia cell line.................................... 45

3. Primary cells....................................... p. 45

a) Bone marrow-derived macrophages (BMDM)........p. 45

b) Transient labeling ....................................... 45

4. Stimulation of BMDMs and MG.................... p. 46

B) Mouse models and in vivo methods.........................p. 46

1. Mouse strains......................................... 46

2. BrM modelling ........................................ 47

3 Tracking of tumor progression........................ p. 47

a) Bioluminescence imaging ......................... p. 47

b) MRT measurement.................................. 47

4. Whole brain irradiation of mice...................... p. 48

5. Final isolation of brain/BrM tissue

and peripheral blood................................... 49 
C) Brain Slice (BrSI) Assay and live cell imaging.................. p. 49

1. Slice generation......................................... p. 49

2. Multi-cellular culture..................................... 50

3. Imaging and analysis..................................... 50

D) Histology on thin and thick mouse brain sections................p. 51

1. HE-staining and immune-histochemistry.................. 51

2. Immunofluorescence of thin brain sections...............p. 51

3. Immunofluorescence of thick brain sections............. p. 52

a) Slice generation and tissue clearing.................p. 52

b) Blocking and staining.................................. 52

c) Confocal imaging ....................................... 52

E) Flow cytometry and FACS...................................... p. 53

1. Preparation of peripheral blood......................... p. 53

2. Preparation of brain tissue................................ 54

3. Flow cytometric analysis................................ 55

4. FACS of immune cells for IF, qPCR and RNASeq...... p. 57

F) RNA isolation, cDNA synthesis and qPCR ................... p. 57

1. RNA isolation.............................................. 57

2. CDNA synthesis............................................ 58

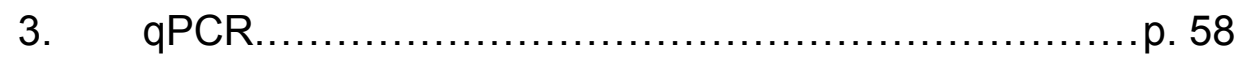

G) RNA-Sequencing ................................................. 59

1. Sample preparation and sequencing....................... 59

2. Downstream data processing.......................... p. 59

3. RNA-Seq data analysis................................ p. 60

a) DESeq2: standard method for DEG generation........p. 60

b) Further downstream analysis (pathway analysis, Venn and Euler plotting)..............p. 60

4. Single Cell RNA-Seq.................................... p. 61

H) Data, analysis and software.................................... 6.62

1. Data sets generated/used in this thesis................. p. 62

2. Analysis, software and packages....................... p. 62

3. Example script for RNA-Seq data analysis............... p. 63-69 
A) Brain metastasis induce changes within the spatial organization of brain-resident cell types and induce activation of glial cells.

B) Immune cell infiltration into BrM p. 73

1. TAMs represent a major stromal compartment of BrM.p. 73

2. Brain Slice Assay and live cell imaging mimics a dynamic BrM TME.

3. Lymphoid cells in breast-to-brain metastasis

p. 78

C) Whole brain radiotherapy (WBRT) affects the BrM TME in a dynamic manner.

1. The primary tumor type drives changes in BrM-associated myeloid cell populations in response to WBRT.

2. Fractionated WBRT slows the recruitment of distinct myeloid cell populations in syngeneic breast-to-brain metastasis

3. WBRT changes the ratio of $\mathrm{CD}^{+}$and $\mathrm{CD} 8^{+}$ T cells in 99LN BrM p. 86

D) RNA-Sequencing reveals molecular responses in BrM-associated immune cells

1. TILs in 99LN-BrM show an activated/exhausted phenotype

2. 99LN-BrM infiltrating myeloid cells upregulate marker to interact with TILs.

3. The myeloid-rich H2030-BrM TME allows exploration of myeloid cell states under different conditions.

4. WBRT slightly changes the transcriptomes of different BrM-associated myeloid cell populations....

5. Single cell RNA-Sequencing reveals TAM heterogeneity before and after WBRT

6. Cross-model comparative analyses of RNA-Seq data reveals BrM-specific transcriptomic profiles and a core BrM gene set of TAM-MG and TAM-MDM. p. 107

7. TAM-MG and TAM-MDM represent two distinct cell types in BrM

E) The complement system in BrM

1. The complement system as a central mediator of BrM-associated inflammation

2. The complement system in human BrM-associated microglia and macrophages 
A) Different types of brain metastasis vary in their immune cell infiltration pattern.

B) Transcriptomic profiles of the tumor microenvironment in brain metastasis

1. Lymphocytes become exhausted within the BrM TME.p. 124

2. Microglia and macrophages represent two distinct populations in BrM.

C) The complement system as central player in instigating inflammation in brain metastasis?...

D) WBRT influences the dynamic TME and only moderately changes TAM transcriptomes.

E) Outlook

VIII References

p. 136

IX List of Publications and Contributions

p. 148

Acknowledgements

p. 151

Declaration / Eidesstattliche Erklärung

p. 154

CV

p. 155 


\section{Abbreviations}

APC antigen-presenting cell

B2B breast-to-brain (metastasis)

BAM border-associated macrophages

BBB blood-brain barrier

BMDM bone marrow-derived macrophage

BLI bio-luminescence imaging / image

BrM brain metastasis

$\mathrm{BrSl} \quad$ brain slice

cDNA complementary DNA

CNS central nervous system

CT computer tomography

CTLA4 cytotoxic T-lymphocyte antigen-4

DC dendritic cells

( $\mathrm{pDC}=$ plasmacytoid $/ \mathrm{cDC}=$ conventional $)$

DEG differently expressed gene

EAE experimental autoimmune-encephalitis

FACS fluorescent-activated cell sorting

FCM flow cytometry

Granu granulocyte

ICB immune-checkpoint blockade

IF immuno-fluorescence

IR ionizing radiation

IT immunotherapy

KPS Karnofsky performance status

L2B lung-to-brain (metastasis)

LM leptomeningeal metastasis

MAC membrane attack complex

MDM monocyte-derived macrophage

MG microglia

MIP maximum intensity projection

Mono monocyte

MRI magnet resonance imaging 
MS multiple sclerosis

NSCLC non-small cell lung cancer

NVU neuro-vascular unit

o/n over night

PCA principle component analysis

RNA ribonucleic acid

RNA-Seq RNA sequencing

RT room temperature

RTH radiotherapy

SARRP small animal radiation research platform

scRNA-Seq single cell RNA sequencing

SN supernatant

SRS stereotactic radiosurgery

TA tumor-associated

TAM tumor-associated macrophages/microglia

TIL tumor-infiltrating lymphocytes

TME tumor microenvironment

tSNE t-distributed stochastic neighbor embedding

WBRT whole brain radiotherapy

WT wild type 


\section{Figures and Tables}

\section{Figures}

Figure 1: Simplified overview of the microenvironment in the brain under homeostatic (I) and BrM (II) conditions.

p. 31

Figure 2: Overview of the main methods applied within this thesis.

p. 39

Figure 3: Whole brain radiotherapy of mice with the SARRP.

p. 48

Figure 4: Overview of the gating strategies for FCM and FACS panels.

p. 56

Figure 5: Single cell RNA-Seq of H2030-associated TAMs experimental approach.

p. 61

Figure 6: Distribution of brain-resident cell types in BrM.

p. 71

Figure 7: Immuno-stainings of microglia/macrophages in BrM.

p. 72

Figure 8: The dynamic myeloid immune cell compartment within the TME of different BrM models.

p. 75

Figure 9: Triple culture brain slice live imaging reveals a highly dynamic microenvironment in the presence of tumor cells.

Figure 10: The lymphoid TME in 99LN breast-to-brain metastasis.

p. 79

Figure 11: The dynamic BrM-associated myeloid immune cell compartment in response to WBRT.

p. 82

Figure 12: Morphologic changes in the TAM population in response to WBRT.

Figure 13: The breast cancer BrM-associated myeloid immune cell compartment in response to WBRT.

p. 85

Figure 14: The breast cancer BrM-associated lymphoid immune cell compartment in response to WBRT.

Figure 15: 99LN-BrM-associated lymphoid cells upregulate markers of activation.

p. $90 / 91$

Figure 16: 99LN-BrM-associated myeloid cells contribute to an immuno-suppressive TME.

p. 94

Figure 17: The $\mathrm{H} 2030$ lung cancer BrM model.

p. 97

Figure 18: Molecular consequences of WBRT-treated H2030-BrM.

p. 100 
Figure 19: Transcriptional changes of irradiated TAMs in H2030-BrM.

p. 102

Figure 20: Single cell RNA-Seq of TAMs in H2030-BrM with and without WBRT.

Figure 21: Multimodal comparison of TAM RNA-Seq data.

p. 109

Figure 22: Functional annotation of commonly regulated genes in TAMs

p. 110

Figure 23: TAM-MGs and TAM-MDMs represent two distinct macrophage populations in BrM.

p. 112

Figure 24: M1 and M2 polarization marker across both TAM populations in different BrM models.

p. 114

Figure 25: The complement system in murine BrM-associated immune cells.

p. 117

Figure 26: The complement system in human BrM-associated microglia and macrophages.

p. 120

\section{Tables}

Table 1: Resource identifier table.

p. 40

Table 2: Overview of tumor cell lines.

p. 44

Table 3: Overview of microglia cell line.

p. 45

Table 4: Components of brain slice medium.

p. 50

Table 5: Primary and secondary antibodies for histology.

p. 53

Table 6: Overview of FCM/FACS antibodies.

p. 55

Table 7: TaqMan probes for qPCR.

p. 59 


\section{Zusammenfassung / Summary}

\section{Graphical Overview}

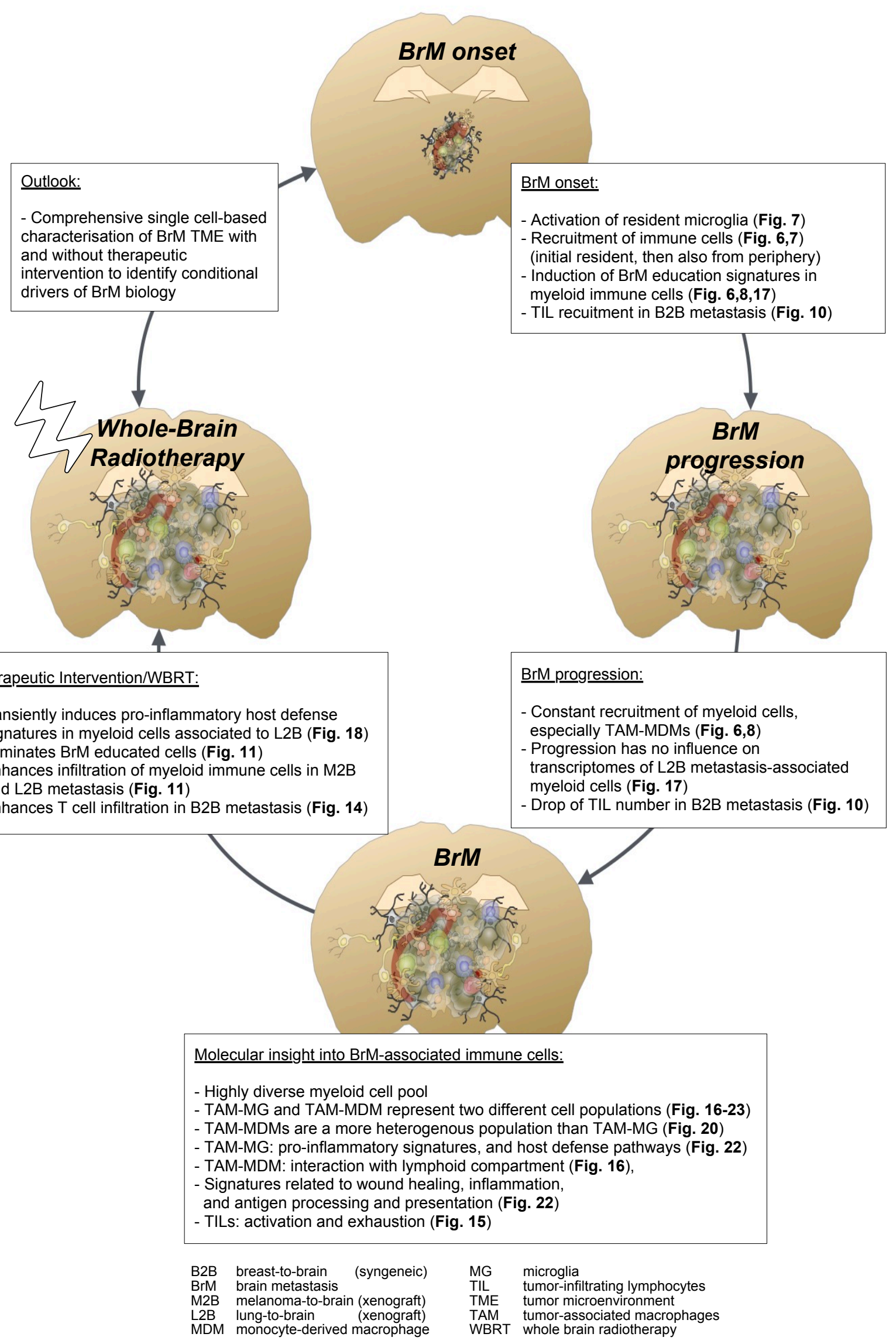




\section{A) Zusammenfassung - deutsche Version}

Krebserkrankungen stellen trotz stetiger Fortschritte in Wissenschaft und Medizin, noch immer eine der häufigsten Todesursachen dar, wobei Tumore des zentralen Nervensystems (ZNS) häufig mit besonders kritischen Prognosen einhergehen. Auch wenn bislang ca. 100 unterschiedliche molekulare Subtypen von primären Hirntumoren klassifiziert wurden, stellen Absiedlungen von Tumoren aus der Peripherie (= Hirnmetastasen, brain metastasis, BrM) den überwiegenden Teil aller Hirntumore dar. BrMs können dabei prinzipiell von jeder Tumorentität hervorgehen und ca. 20 - 40 \% aller Krebspatienten entwickeln im Laufe ihrer Erkrankung BrMs. Allerdings zählen Lungenkrebs, schwarzer Hautkrebs (Melanom), sowie Brustkrebs zu den Entitäten, welche besonders häufig ins Hirn metastasieren. Die Behandlungsoptionen für Patienten mit BrM bewegen sich in einem relativ engen Rahmen. Therapieansätze beinhalten meist neuro- oder radiochirurgische Interventionen und/oder Radiochemotherapie. Als Standardtherapie wird meist auch Ganzhirnbestrahlung (whole-brain radiotherapy, WBRT) angewendet. Mittlerweile kommen jedoch auch zielgerichtete und Immuntherapeutika zum Einsatz. Das Gehirn weist bezüglich seiner Biologie spezifische Eigenschaften auf, wobei zum Beispiel die Blut-Hirn-Schranke dazu dient, einen streng regulierten Stoff- und Zellaustausch zu gewährleisten. Außerdem befinden sich im Hirnparenchym unter normalen, gesunden Bedingungen keinerlei Immunzellen des systemischen Immunsystems. Funktionen der Immunabwehr im Parenchym werden durch hirnresidente Makrophagen, den sogenannten Mikroglia, ausgeübt. Einige Studien der letzten Jahre haben jedoch auch gezeigt, dass sämtliche Arten von myeloiden und lymphoiden Immunzellen in der Peripherie des ZNS, wie zum Beispiel in den Hirnhäuten (Meningen), ansässig sind. Diese Ergebnisse haben entscheidend dazu beigetragen, dass das ZNS nach heutigem Stand nicht mehr als „immun-privilegiert“ angesehen wird.

Dieser Fakt ist auch schon länger bekannt, jedoch nur im Zusammenhang mit einigen neurologischen Erkrankungen, welche zum Einwandern verschiedener Immunzellen führen. Darunter fallen beispielsweise Multiple Sklerose, aber auch Hirntumore.

Da es in vielen Tumoren außerhalb des ZNS verschiedene Ansätze gibt das Immunsystem mit einzubeziehen um langfristige anti-tumorale Effekte zu erzielen, 
besteht ein großes Interesse daran, auch in Hirntumoren die Mikroumgebung, also das Tumor-Stroma, für sich nutzbar zu machen. Interessanterweise entstammt ein Großteil des Wissens über die Mikroumgebung aus präklinischen und klinischen Daten zu primären Hirntumoren. Allerdings ist auch bekannt, dass BrM im Vergleich zu primären Hirntumoren deutliche Unterschiede im Hinblick auf die zelluläre Zusammensetzung aufweisen. So induzieren sie beispielsweise eine stärkere Infiltration von Lymphozyten, weshalb primäre Hirntumore noch schlechter auf immuntherapeutische Ansätze ansprechen. Auch sind einzelne Aspekte, wie die Unterstützung des Tumorwachstums durch hirn-residente Zellen (z. B. Astrozyten oder Mikroglia), bekannt. Insgesamt gab es jedoch bis vor Kurzem noch recht wenig Wissen darüber, wie sich die Mikroumgebung in BrM zusammensetzt oder beispielsweise während der Tumorprogression verändert.

Im Rahmen der vorliegenden Arbeit wurde die Zusammensetzung des Tumorstromas in unterschiedlichen BrM-Modellen deskriptiv untersucht. Hierzu wurden zwei xenotransplantierte und ein syngenes BrM-Mausmodell verwendet. Die Xenotransplantat-Modelle wurden in immun-supprimierten Nacktmäusen (BalbC nude) induziert und umfassten das Melanom-zu-Hirn (M2B) Model H1_DL2, sowie das Lunge-zu-Hirn Model H2030. Beide humane Zelllinien weisen einen hohen Hirntropismus auf. Die murine Brust-zu-Hirn Zelllinie 99LN-BrM wurde in immunkompetenten, wild-typischen BL6 Mäusen intrakardial induziert.

Nach Injektion dauerte es ca. 3 (beide Xenotransplantat-Modelle) bis 5 (99LN) Wochen, bis die ersten Mäuse sicht- und nachweisbare Metastasen ausbildeten. Das Tumorwachstum der Tiere wurde mittels 2 verschiedener Techniken mindestens einmal pro Woche untersucht: der Nachweis und die Wachstumsanalysen der xenotransplantierten Modelle erfolgte über Biolumineszenz-messungen, wohingegen das syngene Model mittels Magnetresonanz-Tomographie (MRT) verfolgt wurde. Anhand der Daten beider Methoden wurden die Tiere schließlich in Gruppen mit kleinen oder großen Tumoren für die weiteren zellulären Untersuchungen, basierend auf histologischen und zytometrischen Methoden, unterteilt.

Immunhistochemie und Immunfluoreszenzfärbungen bestätigten frühere Ergebnisse und zeigten, dass beispielsweise Astrozyten und Mikroglia in Gegenwart von Hirnmetastasen eine aktivierte Morphologie aufweisen, wohingegen Neurone eher unauffällig am Übergang zwischen wachsenden Metastasen und gesundem Parenchym lokalisiert waren. Auffällig war, dass mit zunehmender Tumorgröße eine 
verstärkte Akkumulation einer der Immunzellpopulation zu beobachten war. Bei dieser handelte es sich um die Tumor-assoziierten Makrophagen (TAMs), welche immunhistochemisch durch den pan-Makrophagen Marker IBA1 detektiert wurden. Jedoch war von früheren Arbeiten bekannt, dass diese Population aus mindestens zwei Subpopulationen besteht: den resident-infiltrierenden Mikroglia (MG, TAM-MG), sowie den aus der Peripherie kommenden, Monozyten-entstammenden Makrophagen (TAM-MDM). Durch ihre ähnliche Morphologie und das Fehlen geeigneter Oberflächenmarker taten sich frühere Studien jedoch schwer beide Populationen voneinander zu unterscheiden. Anhand einer Arbeit von vor wenigen Jahren konnte jedoch auf Grundlage von „lineage tracing“ Experimenten der Marker CD49d als spezifisch für MDMs im Kontext von Hirntumor-infiltrierenden myeloiden Immunzellen identifiziert werden, wodurch dessen Verwendung eine zuverlässige Unterteilung beider TAM Populationen erlaubt.

Folglich wurden in dieser Arbeit BrMs aller drei Modelle (H1_DL2, H2030, 99LN) in unterschiedlichen Stadien ihrer Entwicklung mittels Fluoreszenz-zytometrie (=fluorescence cytometry, FCM) untersucht. Durch die Verwendung einer 5-MarkerKombination (CD45/CD11b/Ly6C/Ly6G/CD49d) wurden vier der myeloiden Zellpopulationen genauer charakterisiert: Granulozyten, inflammatorische (infl.) Monozyten, MDM und Mikroglia. Auch wenn das M2B Modell generell nur sehr wenig (weniger als $5 \%$ ) myeloide Zellen aus der Peripherie rekrutierte, zeigten die relativen FCM Daten einen signifikanten Zusammenhang innerhalb von H2030 und 99LN BrMs: während der Tumorprogression steigt der Anteil von Granulozyten und MDMs in beiden BrM Modellen, wohingegen keine verstärkte Infiltration von infl. Monozyten beobachtet wurde.

Um auch transkriptionelle Veränderungen durch und während der Tumorprogression zu charakterisieren, wurden myeloide Zellen aus H2030 und 99LN mittels FCM sortiert und RNA-Sequenzierung durchgeführt. Die Daten zeigen die folgenden, grob zusammengefassten Ergebnisse: BrM-assoziierte infl. Monozyten, MDM und MG weisen im Vergleich zu den gesunden Kontrollen (Blutmonozyten und MG aus tumorfreien Tieren) distinkte, transkriptionelle Veränderungen auf. Untereinander sind sich infl. Monozyten und MDMs ähnlicher, als zu MG. Eines der wichtigsten Ergebnisse war, dass beide TAM-Populationen grundsätzlich unterschiedliche Transkriptome aufwiesen und folglich ihr jeweiliger ontogenetischer Ursprung zu populationsspezifischen, transkriptionellen und damit vermutlich auch funktionell 
unterschiedlichen Veränderungen im Kontext von BrM führt. Dies wurde davor in keiner Arbeit bezüglich BrM untersucht, war jedoch von primären Hirntumoren bekannt. Die genannten Ergebnisse konnten in beiden Modellen (H2030 und 99LN) gezeigt werden und wurden in zwei Manuskripten der Arbeitsgruppe publiziert. Kurz nach der Veröffentlichung der H2030-Daten wurden grundlegende Ergebnisse in humanen Patientenmaterial zweier anderer Forschungsgruppen bestätigt. Im H2030 Model konnte weiterhin gezeigt werden, dass sich die transkriptionellen Muster in den BrM-assoziierten myeloiden Immunzellen während des Tumorwachstums nicht bzw. nur sehr geringfügig änderten. Dies bedeutet, dass die tumor-vermittelte Erziehung einen viel stärkeren Einfluss auf die Gensignaturen der Immunzellen ausübt, als das Tumorwachstum. Um die Gensignaturen beider TAM-Populationen (TAM-MG und TAM-MDM) innerhalb verschiedener Modelle zu vergleichen, wurde in silico ein dritter Datensatz aus dem MDA-Model (xenotransplantiert, Brust-zu-Hirn, Klemm et al., in Revision) hinzugezogen. Diese vergleichenden Analysen zeigten, dass 1.) TAM-MG und TAM-MDM grundsätzlich immer verschiedene Populationen darstellen, 2.) jede Tumorentität spezifische Gensignaturen in TAMs hervorruft und 3.) sich diese aber teilweise überlappen und somit ein BrM-spezifisches Panel an gleich regulierten Genen ergeben. Signalweg-Analysen und funktionelle Annotation dieser BrM-spezifischen, gleich regulierten Gene ergab schließlich auch auf funktioneller Ebene deutliche Unterschiede: während TAM-MG eher proinflammatorische und proliferative Signalwege hochregulieren, wirken TAM-MDM eher durch Wundheilungsprozesse, Umorganisation der extrazellulären Matrix, oder aber auch bei der Antigen-prozession und -präsentation. Außerdem kam es in beiden Populationen zur vermehrten Expression einiger Komplement-Komponenten, ein Signalweg dessen funktionelle Bedeutung für BrM in weiterführenden Studien untersucht werden muss.

Immunfluoreszenzfärbungen in 99LN Gewebe legten außerdem nahe, dass es vor allem MDMs sind, die mit T-Lymphozyten interagieren. Durch die Verwendung eines zweiten FCM-Panels (CD45/CD3/B220/CD4/CD8) konnte schließlich gezeigt werden, dass im lymphoiden Kompartment von 99LN die relative Anzahl an T-Lymphozyten mit voranschreitendem Tumorwachstum abnahm, wohingegen die Anzahl an BLymphozyten leicht anstieg. Die negative Korrelation zwischen Tumorprogression und Menge an T-Zellen wurde bereits in humanen BrM beschrieben und hängt höchst-wahrscheinlich mit dem stetigen Einwandern von immun-supprimierenden 
MDMs zusammen. Interessanterweise jedoch änderte sich die relative Zusammensetzung von $\mathrm{CD}^{+}$und $\mathrm{CD}^{+}$T-Lymphozyten während der Tumorprogression nicht.

Auf molekularer Ebene konnte mittels RNA-Sequenzierung gezeigt werden, dass auch die Haupt-Lymphozyten-Populationen (CD4 ${ }^{+}$und $\mathrm{CD}^{+}$T-Zellen und B-Zellen) in 99LN-BrM deutlich unterschiedliche Gensignaturen im Vergleich zu den Kontrollproben (Blut-Lymphozyten) aufwiesen und CD4 ${ }^{+}$und CD8 ${ }^{+}$T-Zellen typische Aktivierungsmarker wie z. B. Ctla4, Ifng, Lag3, PD1, oder Tcf1 hochregulierten. Die fehlenden anti-tumor Effekte sowie funktionelle Annotation der regulierten Gene legten jedoch nahe, dass die immun-supprimierende Mikroumgebung außerdem zu einem gewissen Überaktivierungs- oder Erschöpfungszustand der T-Zellen führt. Auch wenn sich die relativen Verhältnisse von $C D 4^{+}$und $C D 8^{+}$T-Zellen während der Tumorprogression nicht änderten, konnte mittels FCM gezeigt werden, dass es bereits wenige Tage nach fraktionierter WBRT eine Umkehrung des CD4:CD8Verhältnisses in 99LN-BrM gab. Sowohl drei als auch fünf Tage nach WBRT mit 5 x 2 Gy waren deutlich mehr CD8 ${ }^{+}$als $\mathrm{CD}^{+}{ }^{+}$T-Zellen vorhanden. Die Untersuchung der zellulären und molekularen Auswirkungen von WBRT war ein weiterer wesentlicher Teil dieser Arbeit. Interessanterweise wurden durch fraktionierte WBRT (5 x 2 Gy) nur geringe Veränderungen der zellulären Zusammensetzung innerhalb des myeloiden Kompartments im 99LN-BrM Model hervorgerufen. Dies ließ sich vermutlich darauf zurückführen, dass sowohl hypo- (1 x $10 \mathrm{~Gy})$ als auch klassisch fraktionierte WBRT in diesem Model zu einer transienten Tumor-Stasis führten. Quantitativ waren sowohl die Monozyten, als auch MDM Populationen im Vergleich zu unbehandelten BrM sehr ähnlich. Interessanterweise sah dies in den beiden Xenotransplantat-Modellen anders aus: sowohl in H1_DL2 als auch H2030-BrM bewirkte die fraktionierte WBRT eine stetige Zunahme der MDM Population über die Zeitspanne bis zu 10 Tagen nach der letzten Dosis. Dies war jedoch im M2B-Model relativ gesehen nur gering ausgeprägt und der Anteil an MDM innerhalb der TAMPopulation lag an Tag 10 lediglich bei ca. $10 \%$. Insgesamt veränderte sich die Rekrutierung von myeloiden Zellen durch die fraktionierte WBRT nur mäßig und der Großteil aller BrM-assozierten Zellen bestand aus TAM-MG. Im Gegensatz dazu kam es im H2030 Model nach 5 x 2 Gy WBRT zu einem stetigen und starken Einstrom von vor allem MDM, welche an Tag 10 ca. 40 \% der TAM-Population ausmachten. Auch Monozyten und Granulozyten wurden verstärkt rekrutiert. Sehr interessant war 
im Vergleich hierzu hypo-fraktionierte WBRT, welche in beiden Modellen wieder ähnliche Effekte zeigte: an Tag 3 nach 1 × 10 Gy blieb der Anteil von Granulozyten und Monozyten relativ ähnlich, jedoch wurde vor allem die MDM Population in beiden Modellen stark reduziert, was vermutlich auf eine höhere Strahlungssensitivität zurückzuführen ist. An Tag 5 nach diesem Bestrahlungsschema kam es jedoch wieder zu einem massiven Einstrom von MDM.

Zusammen zeigen diese Daten, dass sowohl die klassische, als auch hypofraktionierte Bestrahlung in den Mausmodellen einen Einfluss auf die Rekrutierung bzw. den Populations-Turnover von TAMs hat.

Auf molekularer Ebene konnte durch RNA-Sequenzierung ein Einblick in die verschiedenen myeloiden Zelltypen im H2030 Modell nach Bestrahlung gewonnen werden. Im Vergleich mit Proben aus unbestrahlten BrMs zeigte keiner der Zeitpunkte nach Bestrahlung einen Einfluss auf die zelluläre Identität der vier Zelltypen. Verglichen mit nicht-behandelten Proben zeigten sich nur geringfügige Änderungen der Gensignaturen von bestrahlten Proben, wobei der Tag 3 nach $1 \mathrm{x}$ 10 Gy Zeitpunkt in allen Zellpopulationen die stärksten Effekte, gemessen an der Anzahl der unterschiedlich regulierten Gene (= differently expressed genes, DEG), zeigte. Interessanterweise zeigte diese Kondition aber gerade in der MDM Population am wenigsten (weniger als 25) DEGs. Insgesamt jedoch hatte jede Kondition ihr eigenes Set an DEGs in den vier myeloiden Zelltypen und funktionelle Annotation der DEGs aller Zelltypen suggerierte, dass hypofraktionierte Bestrahlung zusätzlich zu stress-induzierten Signalwegen wie in der klassischen Fraktionierung, auch verschiedene Signalwege der körpereigenen Immunabwehr hochregulierte. Dies wiederrum impliziert die Rekrutierung von tumor-und behandlungsnaiven Zellen. Da WBRT in diesem Model zu einem verstärkten Einstrom von nicht-behandelten MDMs führte, entstand die Hypothese, dass vor allem der TAM-MDM Pool verglichen mit TAM-MGs eine größere Heterogenität aufweist. Folglich wurde Einzelzell-RNASequenzierung von unbehandelten und behandelten (Tag 3 nach $5 \times 2$ Gy) TAMs durchgeführt. Die Ergebnisse dieses Experimentes bestätigten schließlich zwei Aspekte: 1.) TAM-MDM sind sowohl vor als auch nach fraktionierter WBRT heterogener als TAM-MG und 2.) beide TAM Populationen stellen zwei grundsätzlich verschiedene Zelltypen in Hirnmetastasen dar. 


\section{B) Summary - english version}

Despite constant progress in basic and translational research, cancer is still one of the leading cause of death. In particular, tumors of the central nervous system (CNS) are usually associated with dismal prognosis. Although about 100 distinct subtypes of primary CNS tumors have been classified molecularly, metastases derived from primaries outside the CNS (= brain metastases, BrM) are more frequently observed across brain tumor patients. It is estimated that approximately $20-40 \%$ of all cancer patients will develop BrM during their course of disease, and basically every tumor type is able to metastasize to the brain. Nevertheless, BrM are most frequently derived from primaries of the lung, breast, and skin (melanoma). Treatment options for patients with BrM are very limited, and standard of care therapies include surgery, ionizing radiation (e.g. whole brain radio-therapy, WBRT), and some systemic and immuno-therapeutic approaches.

The brain represents a unique organ, which in part is due to the presence of the blood-brain barrier, a unit of the neuro-vascular interface ensuring tightly regulated exchange of nutrients, molecules, and cells. Furthermore, apart from microglia the brain parenchyma does not harbor other immune cells. Those cells however can be found at the borders of the CNS residing in the meninges, for instance. Based on recent insight on the immune landscape in the CNS, a paradigm shift occurred after which the brain is no longer regarded as immune-privileged but rather immune distinct. The phenomenon of immune cell infiltration has been described before in the context of neurological disorders including Multiple Sclerosis, as well as in brain tumors.

Since the development of immune-therapeutic approaches for tumors outside the CNS that aim to evoke sustainable anti-tumor effects, it became increasingly interesting to understand and harness the immune landscape (= tumor microenvironment, TME) of brain tumors, as well. Interestingly, most of the knowledge about the TME is based on studies of primary brain tumors. However, it is known that BrM compared to primary brain tumors induce a different TME like e.g. the recruitment of much more lymphocytes, which is one of the reasons primary brain tumors are considered immunologically "cold" and poorly respond to immunotherapies. Previous insight into the functional contribution of tumor-associated cells in BrM progression revealed for example that brain-resident cell types (e.g. astrocytes 
or microglia) promote BrM development and outgrowth. However, until recently a comprehensive view on the cellular composition and functional role of the brain metastases-associated TME was missing and little was known how it changes during tumor progression or standard therapy.

Hence, within this thesis it was sought to describe novel aspects of the TME of preclinical BrM models, which include two xenograft and one syngeneic mouse model. BrM was induced via intra-cardiac injection of tumor cells with a high brain tropism. Both xenograft models were based on immuno-compromised nude mice (Balb/c nude) and included the melanoma-to-brain (M2B) model H1_DL2, and the lung-to-brain (L2B) model H2030. In addition the breast-to-brain model 99LN-BrM was used in wild-type mice (BL6), and therefore represented an immuno-competent, syngeneic model. First BrMs could be detected in the xenograft models at 3 weeks after injection, whereas first 99LN BrMs were detected at 5 weeks. BrM development and progression were monitored by bioluminescence imaging once per week in the xenograft models. Tumor progression in the 99LN model was examined by magnetic resonance imaging. Based on the measurement methods, and for further histologic and cytometric experiments, mice were stratified into groups with small or large BrMs, respectively. Some initial immuno-stainings confirmed previous findings, showing that brain-resident cells like astrocytes and microglia become activated in the presence of tumor cells, whereas neurons for example rather give the impression of passive bystanders. Importantly, an accumulation of $\mathrm{IBA} 1^{+}$cells was observed during BrM progression. IBA1 is a pan-macrophage marker that stains all tumorassociated macrophages (TAMs). However previous work suggested that the TAM population consists of at least two main subpopulations in BrM as well: the residentinfiltrating microglia (MG, TAM-MG), as well as the peripheral and monocytic-derived macrophages (TAM-MDM). Since both cell types within the tumor share morphological traits, and due to the lack of markers to distinguish them, an exact discrimination of both cell types was complicated in the past. Recently, an integrative lineage-tracing-based study identified the integrin CD49d as MDM-specific in the context of brain tumor-associated myeloid cells, hence enabling a reliable dissection of both TAM populations in e.g. flow cytometric experiments.

One of the main aims of this thesis was to dissect the myeloid TME in the three different BrM models during tumor progression. Using a 5-marker flow cytometry (FCM) (CD45/CD11b/Ly6C/Ly6G/CD49d) approach, the following cell populations 
were examined in more detail: granulocytes, inflammatory monocytes, MDM, and MG. The M2B model (H1_DL2) induced only weak recruitment of myeloid cells from the periphery. In contrast, analyses of the $\mathrm{H} 2030$ and 99LN BrM model revealed constant recruitment of granulocytes and MDM during tumor progression, whereas the amount of infl. monocytes remained stable throughout BrM progression. In both, $\mathrm{H} 2030$ and 99LN, the stage of BrM development positively correlated with the amount of infiltrating MDM from the periphery.

To further elucidate molecular changes associated to the changing TME in BrM, myeloid cells of $\mathrm{H} 2030$ and 99LN were sorted, and RNA-Sequencing was performed. In summary the data showed: BrM-associated cells, mainly infl. monocytes, TAMMDM, and TAM-MG showed very distinct gene signatures and an altered transcriptome compared to their normal cellular counterparts (blood monocytes, or microglia from tumor-free mice). Monocytes and TAM-MDMs are more similar to each other than to TAM-MG. One of the most important and interesting findings was that both TAM populations showed rather opposing transcriptomes, which most likely results from their different ontological origins. For TAMs of BrM these differences have not been described before, yet were only reported in TAMs of primary brain tumors and probably also lead to fundamental different functional traits. Interestingly, the main findings described here could be revealed in both models (H2O30 and 99LN), and have been published within two studies from our lab. Shortly after the publication of the $\mathrm{H} 2030$ data, two comprehensive studies from other labs validated these dichotomous signatures across the TAM populations in patient samples. Within the H2030 model, results obtained within this thesis revealed that BrM-educated transcriptomes of associated immune cells did not change during tumor progression. This indicates that education upon tumor establishment itself is the driving force leading to transcriptomic changes, whereas tumor progression does not significantly influence the molecular signatures.

To further examine the transcriptional profiles across both TAM populations within different models, data from another set were included in silico. These data were derived from TAM-MG and TAM-MDM obtained from the xenograft B2B model MDABrM. Comprehensive bioinformatic analyses revealed that: 1.) TAM-MG and TAMMDM always represent two distinct TAM populations, 2.) each entity induces tumor type-specific TAM signatures, however 3.) there is also an overlap between these signatures, suggestive of a BrM-specific molecular profile of TAMs. Pathway 
analyses and functional annotation of these BrM-specific genes further suggested fundamental differences. While TAM-MG exert rather pro-inflammatory functions, and upregulated markers related to mitosis, TAM-MDM showed increased expression levels of genes related to wound healing, organization of the extra-cellular matrix, but also antigen processing and presentation. Furthermore, these analyses revealed the upregulation of certain members of the complement pathway across models. However, further examination is required to elucidate functional consequences of complement activation in BrM-associated immune cells.

Interestingly, immuno-fluorescence stainings in 99LN sections further prompted towards MDMs for the interaction with tumor-infiltrating lymphocytes (TILs). In order to gain insight into the lymphoid compartment in BrM, a second FCM panel covering the major lymphoid subsets (CD45/CD3/B220/CD4/CD8) was used and 99LN lesions were examined at different stages during tumor progression. Interestingly, FCM data suggested a decrease of $\mathrm{CD}^{+}$TILs during tumor progression, while the number of B-lymphocytes slightly increased. This negative correlation between tumor progression and $\mathrm{CD}^{+}{ }^{+}$TIL numbers has been shown in patient samples as well, and is most likely due to the steady recruitment of immuno-suppressive MDMs as suggested in the related manuscript. Remarkably however, the relative ratio of CD4 ${ }^{+}$ and $\mathrm{CD}^{+}$TILs did not change during tumor progression. RNA-Seq data of the three major lymphocyte populations (i.e. $\mathrm{CD}^{+}$and $\mathrm{CD} 8^{+}$TILs, B-TILs) revealed that TILs possessed distinct transcriptomes compared to their normal cellular counterpart (i.e. blood lymphocytes). Both CD3 ${ }^{+}$TIL subsets upregulated typical activation marker of T-lymphocytes, for example Ctla4, Ifng, Lag3, PD1, or Tcf1. Annotating differently expressed genes (DEGs) of TILs showed the upregulation of several typical pathways related to activation but also exhaustion. Together with the immunesuppressing TME, this most likely accounts for the lack of anti-tumor effects within 99LN-BrM. Although the ratio of $\mathrm{CD}^{+}$and $\mathrm{CD}^{+}{ }^{+}$TILs associated to either small or large lesions was stable, there was a clear reversion of the relative frequencies of both cell types following whole-brain radiotherapy (WBRT), and already at 3 but also 5 days upon fractionated ( $5 \times 2$ Gy) WBRT, the T-cell population consisted of more $\mathrm{CD}^{+}$than $\mathrm{CD}^{+}$cells.

Another major aim during this thesis was to examine the effects of standard therapy, i.e. WBRT, on the cellular and molecular changes within the TME. In contrast to the lymphoid compartment, WBRT exerted only slight changes within the myeloid 
compartment in 99LN, which most likely was due to the anti-tumor effects of WBRT itself, leading to a transient tumor stasis of 99LN-BrM, and hence prevented more influx of peripheral cells. The number of both, inflammatory monocytes as well as MDMs was very similar to samples from non-irradiated, small lesions at early timepoints following fractionated WBRT. Interestingly, FCM data derived from irradiated xenograft models revealed a different trend within the myeloid compartment. Fractionated WBRT resulted in a continuous increase of the MDM populations until 10 days after the last dosis in both, H1_DL2 and H2030. However this effect was very sparsely in the M2B model, wherein TAM-MDMs represented not more than $10 \%$ of the total TAM population. This revealed that WBRT only moderately affected the recruitment of cells from the periphery in $M 2 B$, and that the majority of all myeloid cells consisted of TAM-MGs.

Contrary, WBRT showed more pronounced effects within BrMs of the H2030 model, and 10 days following $5 \times 2 \mathrm{~Gy}$, about $40 \%$ of all TAMs consisted of MDMs. In addition the recruitment of monocytes and granulocytes was enhanced as well. Very interestingly was the comparison with another treatment scheme, in which one single dose of 10 Gy (= hypo-fractionated WBRT) was applied on BrM-bearing mice. This resulted in a similar effect in both xenograft models, and BrMs examined at d3 upon $1 \times 10$ Gy showed a drastic reduction of the overall myeloid compartment derived from the periphery, most likely due to a higher radio-toxicity. The reduction of the myeloid compartment was only evident at d3 after WBRT. Massive infiltration of MDM resulted in a reversion of this effect already two days later (d5). The observed effects occurred to different extents in each model and were least pronounced in the M2B model. Together, these data show that both, classical fractionated, as well as hypo-fractionated WBRT has the potential to interfere with the recruitment of cells from the periphery and furthermore regulates population turnover.

To unravel the effects of WBRT on the molecular level, RNA-Seq of myeloid cells from H2030-bearing mice was performed. By comparing samples from irradiated lesions with non-irradiated lesions, RNA-Seq data revealed no changes of cellular identity due to the application of WBRT. However, every condition compared to the non-irradiated samples showed a distinct set of differently expressed genes (DEG), which however was below 150 in almost every condition from each cell type.

Interestingly, the d3 time point upon $1 \times 10$ Gy showed the strongest effects in TAMMG, monocytes and granulocytes, based on the number of DEGs. TAM-MDM 
samples from this condition however showed the lowest number of DEGs (below 25). Functional annotation and pathway analyses from all DEGs of all cell types suggested, that despite upregulating stress-induced pathways, a single high dose of WBRT further resulted in higher expression of genes related to host defense mechanisms, compared to $5 \times 2 \mathrm{~Gy}$. Consequently, this proposes the recruitment of a certain amount of tumor-and treatment-naïve cells.

Since WBRT of H2030-BrM-bearing mice enhanced the recruitment of MDMs, this population most likely showed a high amount of heterogeneity, which probably is even higher compared to TAM-MG. To prove this hypothesis, a single cell RNA-Seq approach of non-irradiated and irradiated (d3 upon $5 \times 2$ Gy) TAMs was applied. Finally, these data confirmed two major findings of this thesis: 1.) both TAM populations basically represent two distinct cell types in brain metastases, and 2.) TAM-MDMs from both, treatment-naïve and treated BrMs are a more heterogenous population compared to TAM-MG.

In summary, data of this thesis reveal the dynamics and complexity of the TME in $\mathrm{BrM}$. On the cellular level, one key event in all models was the constant recruitment of monocyte-derived macrophages from the periphery. The MDM population however represents a distinct cell population with most likely non-redundant functions compared to the brain-resident microglia (TAM-MG), although both TAM populations influence the immune-suppressive TME. Education by tumor cells seemed to be a sufficient and long-lasting inducer of transcriptional changes in BrM-associated immune cells across models, while transcriptomes of BrM-associated myeloid cells did not change during tumor progression. Usage of WBRT as therapeutic approach revealed its applicability as immune-modulator in the TME by depleting BrMassociated immune cells, and enhancing the recruitment of naïve cells, e.g. MDMs and $\mathrm{CD}^{+}$lymphocytes. Interestingly, on the molecular level WBRT did not induce massive changes of myeloid cells transcriptomes.

In silico comparison of TAM RNA-Seq data derived from 3 different mouse models furthermore underlined the importance of NGS strategies to unravel BrM-associated transcriptional changes, which highlighted a huge set of unique but also overlapping differently expressed genes. Among the upregulated genes were members of the complement system. The biological importance of complement upregulation in BrMassociated immune cells (mainly in myeloid cells) and if this represents a central player in instigating inflammatory responses in BrM requires further examination. 


\section{Introduction and state of the art}

A) Brain metastasis

Brain metastasis (BrM) represents a serious and challenging clinical issue, and in recent years numbers of BrM patients are even rising. This is due to increasing incidence of different tumor diseases and improved control of primary tumor leading to prolonged survival of patients and consequently longer time windows in which metastasis can develop. In Europe, about $25 \%$ of all death can be attributed to cancer (Hofmarcher et al., 2019). Survival rates of BrM patients are very poor and median overall survival is only a few months. The spreading of malignant cells as one hallmark of cancer (Hanahan and Weinberg, 2000) impairs initial therapy success and is actually one of the most dangerous aspects, accounting for the majority of cancer-related death (Sporn, 1996). BrM occurs in approximately 20 - $40 \%$ of all cancer patients (Fokas et al., 2013, Suh et al., 2020), and the highest incidences can be found in lung cancer, breast cancer and melanoma, whereas basically every tumor type can spread to the brain (Gavrilovic and Posner, 2005, Nayak et al., 2012). Moreover different primaries induce a variable number of lesions within the brain, which is considered to be a disease-specific prognostic factor (Lin and DeAngelis, 2015). Together, BrM represents the most common brain tumor in adults, which exceeds numbers of primary brain cancer patients.

\section{B) Induction of brain metastasis}

Metastatic seeding to the brain represents an inefficient and complex, multi-stage, continuous process with only few cells that disseminate from the primary site successfully performing each step of the metastatic cascade (Gavrilovic and Posner, 2005, Massague and Obenauf, 2016). It further expects cancer cells to enter the brain, to survive and adapt, and (re-) become metabolically active in a very specialized secondary organ. Notably, the biology of several steps of BrM formation could be revealed, which for instance include cancer cell intrinsic (e.g. genomic alterations) (Bos et al., 2009, Brastianos et al., 2015, Shih et al., 2020), or extrinsic (e.g. interaction within the new environment) (Sevenich et al., 2014, Valiente et al., 2014, Priego et al., 2018) traits. Upon extravasation most of the cells die (Kienast et al., 2010), and the remaining cells that establish new lesions encounter a unique microenvironment (figure 1). However, for successful outgrowth tumor cells need to 
achieve sufficient supply of nutrients and oxygen through interaction with the vasculature. The vasculature in the brain consists of a highly complex network of micro- and macro-vessels, all together in close interaction with a variety of different cell types forming the blood-brain barrier (BBB) at the neuro-vascular unit (NVU). This barrier represents the tight control element between the brain and the periphery, and consists of endothelial cells, pericytes, and astrocytes, overall regulating brain homeostasis (Abbott, 2013). While a variety of CNS intrinisic and extrinsic factors has been shown to modulate BBB integrity (Segarra et al., 2021), the BBB in brain tumors is compromised and disrupted (comprehensively reviewed in Arvanitis et al., 2019). Its misuse and misformation in malignancies is due to two basic mechanisms: vessel co-option or (neo-) angiogenesis (Kienast et al., 2010, Plate et al., 2012, Valiente et al., 2014, Arvanitis et al., 2019, Seano and Jain, 2020). In BrM it was shown that different primary tumor cell types grow either in a co-optive manner or induce angiogenesis. Lung cancer cells massively instigate angiogenesis, whereas melanoma cells grow in a co-optive manner always close to pre-existing vasculature (Kienast et al., 2010). Apart from interacting with vessels in order to ensure the tumors own further existence, tumor cells encounter a variety of brain-resident cell types upon extravasation and colonization of the brain parenchyma.

\section{C) Brain metastasis microenvironment - brain-resident cells}

Certainly the cell type one most likely thinks of when talking about the brain is neurons. Neurons represent a heterogeneous cell population that colonize each region of the CNS and mediate all conscious and unconscious types of behavior and action. However, in the BrM situation neurons have long been described as bystanders without active contribution to BrM biology. Seano et al. described neuronal loss and impaired neuronal function in primary and metastatic brain tumors, as consequences of solid stress (Seano et al., 2019). Surprisingly, another recent finding additionally indicates a critical contribution of neurons and especially their synapses to the outgrowth and progression of breast-to-brain metastasis (Zeng et al., 2019) by interfering with glutamate signaling, hence shifting the view of neuronal contribution to BrM biology. In addition, breast cancer cells themselves within the brain have been shown to adapt to their novel environment by changing the expression of e.g. members of GABA metabolism, which enables them to integrate into the neural niche (Neman et al., 2014). This interaction of tumor cells with 
neuronal networks seems to be conserved within primary and secondary brain tumors (Venkataramani et al., 2019, Venkatesh et al., 2019).

To date, the role of oligodendrocytes, a cell type implicated in ensuring neuronal function, has not been investigated in BrM and it remains to be elucidated whether oligodendrocytes actively or passively influence BrM development and/or progression. This is in contrast to previous findings in primary brain tumors, where oligodendrocytes or their precursors have been found tumor-promoting (Huang et al., 2014, Hide et al., 2018). Nevertheless, their contribution to different other brain diseases is becoming increasingly recognized, for instance in Multiple Sclerosis (MS) or its preclinical mouse model, experimental autoimmune-encephalitis (EAE) (Jäkel et al., 2019), thus maybe influencing BrM as well.

The second glial cell type within the brain is astrocytes (figure 1). Since astrocytes represent the most abundant, non-neuronal cell type within the CNS parenchyma, tumor cells encounter their presence and interaction very early following extravasation. Their roles and multiple functions in BrM induction, progression, and therapy resistance has been extensively described (see. reviews Sofroniew and Vinters, 2010, Wasilewski et al., 2017). Doron et al. (2019) reported that astrocytederived CXCL10 attracted melanoma cells into the brain, thus making astrocytes key players in tumor cell recruitment to the CNS (Doron et al., 2019). On the other hand, upon extravasation tumor cells for example initially undergo astrocyte-triggered cell death, a mechanism they circumvent by activating inhibitors against apoptosis (Valiente et al., 2014). Generally, astrocytes react towards all kind of insults in the brain, collectively referred to as astrogliosis, which includes distinct cellular and molecular changes (Sofroniew and Vinters, 2010, Liddelow and Barres, 2017). Following extravasation, brain-tropic tumor cells come into direct cellular contact with astrocytes, which was shown to enhance BrM in various ways via connexindependent pro-tumorigenic functions (Stoletov et al. 2013, Chen et al., 2016). Moreover protection from chemotherapy was further described as a result of this cellcell interaction (Lin et al., 2010, Kim et al., 2011). In BrM, astrogliosis is induced early and delineates malignant cells from healthy parenchyma, which in turn is attributed to neuro-protection (Wasilewski et al., 2017, Schulz et al., 2019). Similar as in homeostatic conditions, astrocytes also interact with a variety of cell types in their environment (Khakh and Sofroniew, 2015), where each of them can release mediators of astrogliosis (Sofroniew 2009), whose outcome is regulated in a time- 
and context-specific manner (Colombo and Farina, 2016). For BrM the probably most interesting interaction is with microglia (MG). While $M G$ represent the sole immune cell type within the parenchyma, basically every other immune cell type can be found in the border-associated regions, e.g. the meninges (Korin et al., 2017, Mrdjen et al., 2018) (figure $1 \mathrm{I}$ ).

Microglia as a special type of phagocyte are yolk sac-derived and populate the brain parenchyma together with macrophages associated to the CNS border (BAMs), early in embryonic development (Ginhoux et al., 2010, Utz et al., 2020) (figure 1 I). During neuro-inflammatory conditions, MGs become reactive and induce a distinct phenotype of astrocytes, thereby together probably influencing the outcome of a variety of diseases (Liddelow et al., 2017). It was shown that a small subpopulation of BrM-associated astrocytes is partially responsible for the induction of an immunosuppressive environment influencing innate (e.g. MG) and acquired (e.g. CD8 ${ }^{+}$ tumor-infiltrating lymphocytes, TILs) immunity (Priego et al., 2018), thereby fostering tumor growth.

Microglia are evolutionary conserved (Geirsdottir et al., 2019), and collectively induce a core set of genes in response to different CNS insults (Friedman et al., 2018). This, among other reasons, results from their tremendous sensing and scavenging abilities throughout the parenchyma, since they are equipped with a specific sensome consisting of in total 100 genes (Hickman et al., 2013). Several of those genes (e.g. Cx3cr1, C1qa, Fcrls, Hexb, P2ry12, Tmem119, Tgfbr1, Trem2) belong to a highly specific MG consensus profile, which delineates MG from other brain-resident cells or peripheral monocytes (Hickman et al., 2013; Butovsky et al., 2014). Their plasticity during disease was further revealed under various neuro-degenerative, and neuroinflammatory conditions, in sum highlighting "disease-associated microglia" (DAM) pattern, or the proliferation under inflammatory conditions (e.g. in MS) (Butovsky et al., 2014, Keren-Shaul et al., 2017, Ajami et al., 2018, Jordao et al., 2019, Zhou et al., 2020). Nevertheless, brain tumor-associated MGs are known to crucially influence primary brain tumor biology (Sevenich, 2018, Gutmann and Kettenmann, 2019), while the knowledge about their contribution to BrM is rather scarce. Preclinical studies suggest that MG quickly respond to invading tumor cells by fulfilling host defense functions, which are exploited afterwards to support tumor cell invasion (Lorger et al., 2010, Chuang et al., 2013, Qiao et al., 2019). Consequently, initial protection mechanism are circumvented and subsequently exploited by tumor 
cells themselves to integrate into their novel niche (figure 1), which is further shaped via immune cell recruitment. The infiltration rates of peripheral immune cells to BrM are much higher compared to primary brain tumors, which its majority of immune cells consists of MG (Klemm et al., 2020).

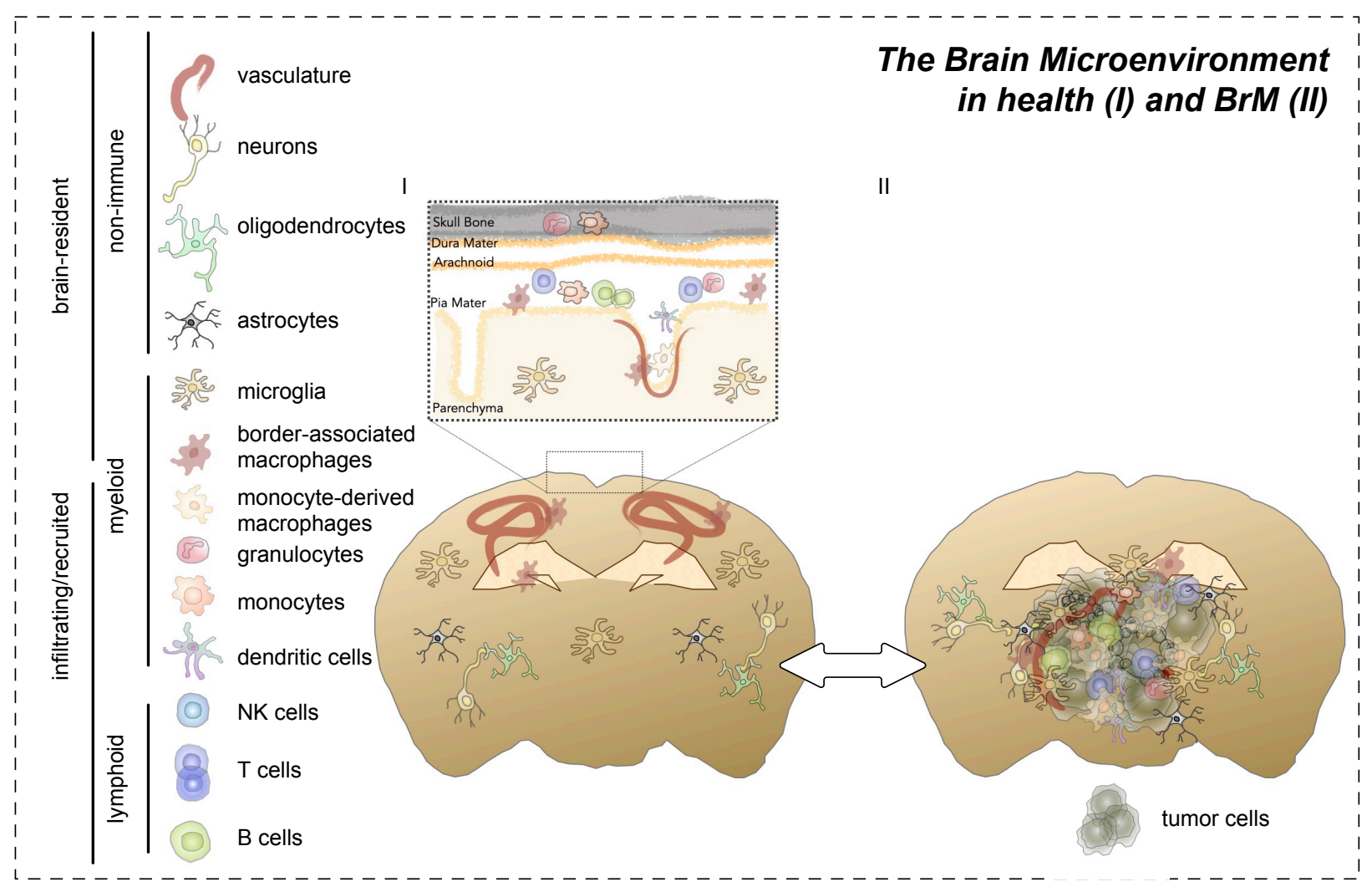

Figure 1: Simplified overview of the microenvironment in the brain under homeostatic (I) and BrM (II) conditions. The BrM-TME consists of brain-resident, and recruited, non-immune, and immune cells.

D) Brain metastasis microenvironment - recruited cells

Brain tumors in general induce massive infiltration of basically every systemic immune cell type from the periphery (Quail and Joyce, 2017), resulting in fundamental changes of the brain's cellular landscape (figure 1II). The extent of recruitment of immune cells seems to be higher in brain tumors compared to neurodegenerative diseases (Sevenich, 2018). In cancer research a major focus has been directed towards tumor-associated macrophages (TAMs), since they are known to critically influence tumor progression and therapeutic outcome (Noy and Pollard, 2014, Mantovani et al., 2017). A scheme that is frequently used classifies TAMs into M1 (pro-inflammatory, anti-tumoral) and M2 (anti-inflammatory, wound-healing, protumoral) phenotypic functions, based on various activation states and cytokine 
secretion profiles. However, this classification is rather simplified since their in vivo states are far more plastic and rather within the continuum of the above-mentioned states (extensively examined and reviewed here e.g. Murray, 2017). Importantly, they critically contribute to intra-cranial tumor biology (Sevenich, 2018, Gutmann and Kettenmann, 2019), and their depletion resulted in prevention of BrM outgrowth (Qiao et al., 2019). Similar to other neuro-degenerative and -inflammatory diseases (Ajami et al., 2018, Mrdjen et al., 2018, Jordao et al., 2019), brain tumors recruit a considerable amount of myeloid cells, mostly of the mononuclear phagocytic system. While MG fuel the TAM pool (TAM-MG) initially, monocyte-derived macrophages (TAM-MDMs) further enrich the developing BrM-TME. Together, both TAM populations represent the most abundant stromal cells within brain tumors and comprise up to $30-50 \%$ of the tumor mass in primary (Pyonteck et al., 2013), or secondary (Sevenich et al., 2014) brain tumors. While the differences (e.g. on the transcriptomic level) between parenchymal $M G$ and those infiltrating from the periphery have been extensively described under various CNS-diseased conditions (comprehensively reviewed in: Böttcher and Priller, 2016, Sevenich, 2018), little was known for the brain tumor situation. Several recent studies investigated the composition of the TAM population within primary brain tumor models (Pyonteck et al., 2013, Muller et al., 2015, Bowman et al., 2016, Chen et al., 2017). Despite differences in the amount of recruited macrophages, it increasingly became clear that both macrophage populations represent dichotomous cell types with probably opposing functions in primary brain tumors (Bowman et al., 2016, Sevenich, 2018). Notably, but only shown for primary brain tumors, there seems to be a consensus about alternating transcriptomes, wherein MG for instance downregulate homeostatic marker (e.g. Cx3cr1, P2ry12, Tmem119), and show rather pro-inflammatory profiles, whereas TAM-MDMs partially upregulate those markers, but exert functions related to immune-suppression, and wound healing thereby fostering tumor growth (Bowman et al., 2016, Sevenich, 2018, Sankowski et al., 2019). Noteworthy, in both, mice and human different localization of the two TAM types has been described (Chen et al., 2017, Darmanis et al., 2017). This underlines the importance of a careful characterization of TAM populations within brain tumors, in order to fully understand functional consequences on tumor progression. In addition, another recent study revealed BAMs as contributing to the local TAM pool, as well (Guldner et al., 2020). However until recently, it was not known if those findings from primary brain tumors 
hold true in the BrM situation. Having carefully characterized the transcriptomic differences between both major macrophage populations in a mouse model of lungto-brain metastasis (Schulz et al., 2020), other studies confirmed these findings in patient samples and preclinical models on the transcriptional and protein level (Friebel et al., 2020, Guldner et al., 2020, Klemm et al., 2020). The studies from Friebel et al. (2020) and Klemm et al. (2020) comprehensively revealed a detailed cellular and molecular immune landscape of primary and secondary brain malignancies. Especially the BrM TME possesses a transcriptionally and phenotypically heterogeneous mononuclear phagocyte population (TAM-MG/TAMMDM/and TA-Monocytes). The composition of TAM-MDM subtypes further exhibited considerable differences within BrM derived from distinct primary tumor entities. Together the authors also demonstrated that the myeloid compartment in BrM furthermore consists of granulocytes, as well as at least three different dendritic cell (DC) populations (pDC, cDC1, cDC2) (figure 1 II) (Friebel et al., 2020, Klemm et al., 2020). While the specific pro- or anti-tumoral functions and contributions of DCs to $\mathrm{BrM}$ remains to be elucidated, it is tempting to speculate that their recruitment serves to fulfill functions related to antigen presentation thereby mediating adaptive immune responses. DCs increasingly gain attention in research of various types of cancer, since they harbor great potential to enhance immunotherapy (Wculek et al., 2019). Noteworthy, they might infiltrate rapidly since they reside in the CNS periphery (e. g. meninges) during homeostasis (Korin et al., 2017, Mrdjen et al., 2018). This is further supported by a study from Jordao et al. showing that DCs rapidly infiltrate under neuro-inflammatory conditions, and execute a more efficient interaction with $T$ cells than MG or BAMs, which however are also capable of doing so (Jordao et al., 2019). $T$ cells as part of the adaptive immunity are present within the periphery of the homeostatic CNS (Korin et al., 2017, Mrdjen et al., 2018), and have been found among B lymphocytes and NK cells to infiltrate various types of brain tumors (Stevens et al., 1988). The TME of all types of BrM harbors TILs (e.g. CD3 ${ }^{+}$cells), although with variable pattern of infiltration and more frequently in BrM derived from melanoma compared to other primary tumor types (Berghoff et al., 2014, Harter et al., 2015, Berghoff et al., 2016). T cell numbers negatively correlate with the size of BrM (Harter et al., 2015), implicating BrM or BrM-TME-intrinsic mechanisms in regulating $T$ cell numbers, thereby escaping immune responses. However, their mode of infiltration is still under debate. Recent studies looking into neuro- 
inflammatory conditions suggest the entry via perivascular routes (Jordao et al., 2019). However, multiple routes seem possible depending on the side of CNS insult. Another recent study in primary brain tumor focused on the meningeal lymphatic vasculature (Song et al., 2020), which was re-described not long ago (Aspelund et al., 2015, Louveau et al., 2015), and was even shown pivotal during neuroinflammation in MS (Louveau et al., 2018). In comparison to primary brain tumors, BrM overall induce the infiltration of higher numbers of lymphocytes, which however mainly consist of T cells and fewer B and NK cells (Friebel et al., 2020, Klemm et al., 2020).

In summary, there has been huge progress within the last years in order to understand the microenvironment of brain tumors and in particular metastasis. Although there has been great effort in further characterizing the BrM TME, treatment options for patients remain very limited, which for instance is due to failure of successful immuno-therapeutic approaches. In this regard, novel strategies which combine standard of care with more targeted therapies are emerging, yet there is still a huge gap of knowledge on how to best combine such treatment modalities.

\section{E) Brain metastasis: diagnosis and treatment}

The invention and development of diagnostic procedures like magnet resonance imaging (MRI) has significantly improved the clinical parameters determining the current situation of patients. For diagnosis and follow-up, MRI together with computer tomography (CT) are the approaches of choice in BrM patients, which allow the detection of clearly delineated BrM lesions, having a sharp border to normal surrounding brain tissue (Lin and DeAngelis, 2015, Soffietti et al., 2017). In addition, neuropathological examination of biopsied tissue might be of further advantage for diagnostic procedure, if available. Nevertheless, due to their complexity BrMs are no longer considered a single entity across patients, but a special site of metastatic disease, assessed and managed in the context of the patients overall treatment options and the origin of the primary tumor. In addition, the most appropriate definitive therapy is selected based on the number, size, and location of BrM (Lin and DeAngelis, 2015). The treatment of patients with BrM includes directed strategies against the tumor and supportive treatment to help reduce symptoms (Lin and DeAngelis, 2015). Stratifying patients is further complicated by different therapeutic options and its combinations, however distinct meta-analyses and a systematic 
review of literature proposes four major factors influencing treatment decisions and patients survival: age, extracranial metastases, number of BrM, and the Karnofsky performance status (KPS) (Sperduto et al., 2020), which is a staging system and describes the symptom-related functional status of a patient.

Harnessing the full power of modern medicine, current treatment approaches include the following five aspects: surgery, chemotherapy, molecularly targeted therapeutics, immunotherapy, and ionizing radiation in different ways of application (Sperduto et al., 2020, Suh et al., 2020). Neuro-surgery as classical method is usually considered for patients with a few or only one BrM and a good prognosis, and immediately improves the overall patient situation (Lin and DeAngelis, 2015). Other traditional approaches like systemic chemotherapies have been found little effective due to the presence of the BBB, which however has changed over the last years, in which multiple strategies have been developed/optimized to overcome BBB-mediated restrictions (Arvanitis et al., 2019, Suh et al., 2020). Drivers of these approaches are studies investigating the genomic alterations in cancer cells metastasized to the brain, which showed considerable molecular alterations compared to the tumor they originate from. On the other hand, the percentage of BrM patients that are susceptible for targeted therapies is very low (Brastianos et al., 2015, Valiente et al., 2018). Immunotherapies (IT) or immune checkpoint blockade (ICB) approaches make use of harnessing different cells of the immune system to induce long lasting responses in cancer patients (Sharma et al., 2017). Although TILs are recruited to and commonly found across BrM (Berghoff et al., 2014), the prognostic value of dense TIL infiltrates in BrM is disputed, since one study found a more favorable outcome in patient survival (Berghoff et al., 2016), while Harter et al. (2015) did not find a correlation within a large, mixed BrM cohort they analyzed.

One example and one of the first studies specifically focusing on the application of ICB for patients with BrM was conducted in melanoma BrM patients by means of ipilimumab - a monoclonal antibody against CTLA4 (Margolin et al., 2012). CTL4, or cytotoxic T-lymphocyte antigen-4, acts as a molecular brake that dampens $\mathrm{T}$ cellmediated effects. Hence blockade with ipilimumab reactivates effector cell functions. The study by Margolin et al. showed, that administration of this ICB was effective in approximately $25 \%$ of patients with asymptomatic BrM, and in about $10 \%$ of those with symptomatic disease (Margolin et al., 2012). Sadly however the application of ICB within tumors usually goes along with a plethora of tumor cell extrinsic and 
intrinsic mechanisms responsible for resistance (Sharma et al., 2017), and in th brain has to be performed within an established immuno-suppressive TME (Schulz et al., 2019). Hence, further treatment options or combinations are in urgent need in order to increase therapeutic efficacy via lifting immune suppression, or sensitizing for further immune modulation, for instance (Schulz et al., 2019, Sevenich, 2019, Lehrer et al., 2020). There are several clinical data supporting the combinatorial treatment approach (Lehrer et al., 2020). One example would be the combination with radiotherapy $(\mathrm{RTH})$, which alone has been the gold standard of treating brain tumor patients. RTH can be sub-divided into two major forms of application: whole brain radio-therapy (WBRT), and stereotactic/focal radio-surgery (SRS). Regardless of the exact IT which was applied, one recent retrospective meta-analysis showed a clear survival benefit for melanoma BrM patients, which received IT in combination with WBRT or SRS, compared to either RTH modality alone (Moyers et al., 2021). SRS represents an interesting approach, because it combines advantages from surgery with irradiation, and it involves the delivery of one high dose to a prespecified area while sparing the surrounding, normal tissue (Lehrer et al., 2020). Despite SRS might be more advantageous for distinct clinical settings (e.g. only few lesions, good overall performance), and in order to avoid long-term cognitive deficits (McTyre et al., 2013), WBRT is the treatment of choice and remains an important component in managing patients with BrM, especially with widely disseminated metastases (McTyre et al., 2013, Lehrer et al., 2020). Interestingly, one meta-analysis however found the combination of WBRT plus SRS was not improving overall survival (Tsao et al., 2012a). Typically, WBRT is delivered in a fractionated manner, e.g. a total of $30 \mathrm{~Gy}$ within 10 fractions or 20 Gy within 5 fractions (Lin and DeAngelis, 2015, Soffietti et al., 2017, Suh et al., 2020). A comprehensive meta-analysis examining different treatment schemes suggests other variants of treatment delivery were not beneficial for overall patient survival (Tsao et al., 2012b). Hence treatment options for patients considering its possible combinations seem rather diverse, and partially contradictory. Ongoing clinical trials (www.clinicaltrials.gov; NCT04277403) for instance examine the efficacy of SRS vs. HA-WBRT in patients with multiple (4 - 15) BrM. HA-WBRT is a special form of WBRT where radiation of the hippocampus $(H)$ is avoided $(A)$, since it is harboring neural progenitor cells responsible for renewal of certain neuronal and glia cell populations. 
While the outcome of treatments are usually measured based on criteria including patients performance, survival, or local control of tumor burden, it seems interesting that there is not that much known about how standard therapies actually influence not only the tumor itself, but rather its entire ecosystem. Since several studies including brain tumors, suggest that radiation harbors great potential to modulate the whole TME (Sevenich, 2019), this in turn would increase our understanding of how to boost anti-tumor immunity by application of standard of care. In mouse models of primary brain tumors for example, targeting the myeloid compartment of the TME has been shown to prolong survival of mice in combination with irradiation by inhibiting tumor-promoting functions (Stafford et al., 2016). 


\section{Aims and questions}

Given the scarce knowledge of the TME in BrM until recently and the above mentioned complexity of patient's treatment modalities, this thesis aims to unravel alterations in the cellular and molecular composition of the complex BrM TME and its dynamic changes during tumor progression and following whole-brain radiotherapy (WBRT) as standard of care. Since tumor-associated macrophages (TAM) have been shown to critically influence tumor development, progression, and therapeutic resistance, a major focus of this work was the examination of the different molecular aspects of the mononuclear phagocytes associated to BrM under distinct conditions, i.e. during BrM progression and in response to WBRT.

Specific questions to address include:

1.) How does the brain microenvironment changes upon BrM onset?

2.) How does the BrM TME changes during BrM progression on the cellular and molecular level with respect to the myeloid and lymphoid compartment?

3.) What are the cellular and molecular consequences of WBRT for the BrM TME?

4.) What are the quantitative contributions of the major TAM populations (TAM-MG and TAM-MDM) in different BrM models?

5.) How do they change during BrM progression and in response to WBRT?

6.) Do both TAM populations also transcriptionally represent distinct cell types within $\mathrm{BrM}$, and what are their putative functions within the TME?

7.) Is RNA-Seq of BrM-associated immune cells yielding transcriptional targets, which might be relevant for inflammation? 


\section{Material and Methods}

In order to gain deeper insight into the changing microenvironment within the brain upon BrM, three main methodological approaches were used during this work, including: histology, Flow Cytometry (FCM) and RNA-Sequencing (figure 2). The following part contains detailed description of all materials (table 1 - table 7 ) and methods used during this work.

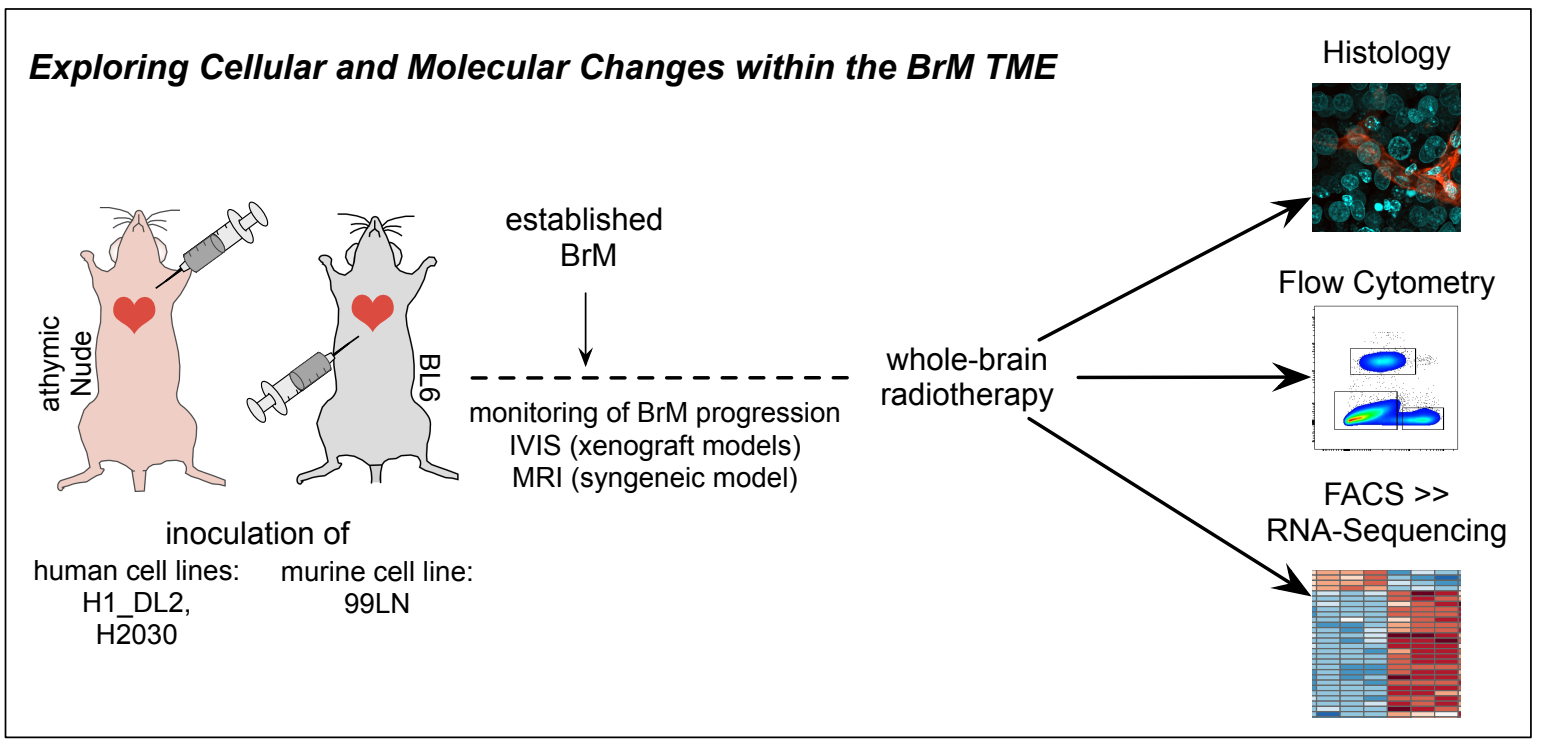

Figure 2: Overview of the main methods applied within this thesis in order to comprehensively dissect the molecular and cellular changes of the BrM TME in various mouse models. 
Table 1.: Resource identifier table

\begin{tabular}{|c|c|c|}
\hline Reagent or Resource & Source & Identifier \\
\hline \multicolumn{3}{|c|}{ Experimental models: mice strains } \\
\hline C57BI/6J & $\begin{array}{l}\text { Charles River Laboratories } \\
(\mathrm{CRL})\end{array}$ & 000664 \\
\hline CX3CR1-GFP & $\begin{array}{l}\text { Jung et al., } 2000 \\
\text { (bred in-house) }\end{array}$ & e.g. JAX \#005582 \\
\hline $\begin{array}{l}\text { CAnN.Cg-Foxn1nu/Crl } \\
\text { (BalbC-nude) }\end{array}$ & $\begin{array}{l}\text { CRL or bred in-house } \\
\text { (het female } \mathrm{x} \text { hom male) }\end{array}$ & CRL \#490 \\
\hline GFAP-Cre & Garcia et al., 2004 & $\begin{array}{l}\text { obtained as GFAP-CrexAxIFL/FL } \\
\text { from C. Rothlin, Yale, US }\end{array}$ \\
\hline $\begin{array}{l}\text { Gt(ROSA)26Sor }{ }^{\text {tm14(CAG- }} \\
\text { tdTomato)Hzw }\end{array}$ & Madisen et al., 2010 & JAX \# 007914 \\
\hline \multicolumn{3}{|c|}{ Experimental models: cell lines } \\
\hline 99LN-BrM2 & Bowman et al., 2016 & $\begin{array}{l}\text { obtained from J. Joyce, University } \\
\text { of Lausanne, Switzerland }\end{array}$ \\
\hline EOC2 (microglia) & ATCC, US & CRL-2467 \\
\hline H1_DL2 & Sundstrom et al., 2013 & $\begin{array}{l}\text { obtained from F. Thorsen, } \\
\text { University of Bergen, Norway }\end{array}$ \\
\hline $\mathrm{H} 2030$ & Nguyen et al., 2009 & $\begin{array}{l}\text { obtained from J. Massague, } \\
\text { MSKCC, NY, USA }\end{array}$ \\
\hline \multicolumn{3}{|l|}{ Bacterial and virus strains } \\
\hline pHR'-SBW lentiviral vector & Brendel et al., 2014 & in-house \\
\hline \multicolumn{3}{|c|}{ pHR- lentiviral vector containing BFP sequence under SFFV promoter } \\
\hline \multicolumn{3}{|l|}{ Assays and kits } \\
\hline Brain Tumor Dissociation Kit & Miltenyi, Germany & $130-095-939$ \\
\hline cDNA synthesis Kit & Invitrogen, US & 4368814 \\
\hline Cell tracker Reagent & Life Technologies, US & \\
\hline Red & & C34552 \\
\hline Blue & & $\mathrm{C} 2110$ \\
\hline Green & & C7025 \\
\hline Myelin Removal Beads & Miltenyi, Germany & $130-096-433$ \\
\hline TaqMan Mastermix & Applied Biosystems, US & 4364338 \\
\hline TaqMan probes $\rightarrow$ Table 7 & Life Technologies, US & \\
\hline \multicolumn{3}{|l|}{ Chemicals } \\
\hline Chloroform & Sigma-Aldrich, Germany & 288306 \\
\hline D-luciferin & BioCAT, Germany & 7903-5-BV \\
\hline DAKO mounting medium & Agilent Technologies, US & S302380-2 \\
\hline DAPI & Sigma-Aldrich, Germany & D9542 \\
\hline DEPC-water & Fisher Scientific, US & AM9916 \\
\hline EDTA $(0.5 \mathrm{M})$ & AppliChem, Germany & A4892 \\
\hline Glycoblue co-precipitate & Thermo Fisher Scientific, US & AM9516 \\
\hline Histofix (contains $4 \%$ PFA) & Carl Roth, Germany & P087.3 \\
\hline Isoflurane & Piramal Critical Care, Germany & 09714675 \\
\hline Isopropanol & Carl Roth, Germany & 9781.2 \\
\hline Ketamin (Ketavet) & Pfizer, US & 3151811 \\
\hline L-glutamine (200 mM) & Life Technologies, US & 25030081 \\
\hline O.C.T. Compound & bought via VWR, US & 4583 \\
\hline Penicilin/streptomycin & Life Technologies, US & 15140130 \\
\hline Plasmocin & InvivoGen, US & ant-mpp, ant-mpt \\
\hline Triton $\mathrm{X}-100$ & Merck, Germany & T8787 \\
\hline TRIzol & Thermo Fisher Scientific, US & 15596018 \\
\hline TRIzol LS & Thermo Fisher Scientific, US & 10296028 \\
\hline Trypsin-EDTA (0.05 \%) & Life Technologies, US & 25300054 \\
\hline X-Clarity mounting medium & Biozym Scientific, Germany & 874302 \\
\hline
\end{tabular}




\begin{tabular}{|c|c|c|}
\hline Xylazin/Rompun (2 \%) & Bayer, Germany & 1320422 \\
\hline \multicolumn{3}{|l|}{ Media and buffer } \\
\hline Advanced DMEM & Life Technologies, US & 12491023 \\
\hline DMEM & Life Technologies, US & 21969-035 \\
\hline HBSS & Life Technologies, US & 14175129 \\
\hline PBS (1x)/(10x) & Life Technologies, US & $14190136 / 14200067$ \\
\hline $\begin{array}{l}\text { Red Blood Cell Lysis Buffer } \\
\text { (RBC), } 10 x\end{array}$ & BD Bioscience, US & 555899 \\
\hline RPMI & Life Technologies, US & 12633012 \\
\hline \multicolumn{3}{|l|}{ Proteins and antibodies } \\
\hline \multicolumn{3}{|c|}{$\begin{array}{l}\text { primary and secondary antibodies for histology see Table 5, } \\
\text { directly labeled primary antibodies for FCM/FACSort see Table } 6\end{array}$} \\
\hline BSA (bovine serum albumin) & JacksonImmunoResearch, US & $001-000-162$ \\
\hline FBS (fetal bovine serum) & PAN Biotech, Germany & $3302-0100509$ \\
\hline Fc block & BD Bioscience, USA & 553141 \\
\hline Non-essential amino acids & Gibco, US & 11140035 \\
\hline recomb. mouse (rm) M-CSF1 & R\&D Systems, US & 416-ML-010 \\
\hline recomb. mouse (rm) IL34 & R\&D Systems, US & 5195-ML-010 \\
\hline recomb. mouse (rm) TGF-ß & R\&D Systems, US & 7666-MB-005 \\
\hline \multicolumn{3}{|l|}{ Plastic consumables } \\
\hline Cell scraper $(25 \mathrm{~cm})$ & Sarstedt, Germany & 831.830 \\
\hline cell strainer $(40 \mu \mathrm{m})$ & Greiner Bio-One, Austria & $5420-40$ \\
\hline cell strainer $(70 \mu \mathrm{m})$ & Greiner Bio-One, Austria & $5420-70$ \\
\hline FACS tubes & Sarstedt, Germany & 551.579 \\
\hline Microscope Cover Slips & Karl Hecht, Germany & $1001 / 20$ \\
\hline Microtubes (1.5 mL) & Sarstedt, Germany & 72.706 \\
\hline Microtubes (2 mL) & Sarstedt, Germany & 72.691 \\
\hline Pasteur pipettes (150 mm) & Roth, Germany & 4518 \\
\hline Pasteur pipettes $(230 \mathrm{~mm})$ & Roth, Germany & 4522 \\
\hline Pipettes (5 mL) & Greiner Bio-One, Austria & 606180 \\
\hline Pipettes (10 mL) & Greiner Bio-One, Austria & 607180 \\
\hline Pipettes (25 mL) & Greiner Bio-One, Austria & 760180 \\
\hline $\begin{array}{l}\text { Pipet tips }(0.1-2.5 \mu \mathrm{l}, 0.5- \\
10 \mu \mathrm{l}, 2-20 \mu \mathrm{l}, 10-100 \mu \mathrm{l} \\
20-200 \mu \mathrm{l}, 100-1000 \mu \mathrm{l})\end{array}$ & Eppendorf, Germany & epT.I.P.S., reloads \\
\hline $\begin{array}{l}\text { SuperFrost Plus } \\
\text { microscope slides }\end{array}$ & Thermo Scientific, US & J1800AMNZ \\
\hline T25 cell culture flasks & Greiner Bio-One, Austria & 958175 \\
\hline T75 cell culture flasks & Greiner Bio-One, Austria & 960175 \\
\hline T175 cell culture flasks & Greiner Bio-One, Austria & 660175 \\
\hline $0.45 \mu \mathrm{m}$ filter & Sarstedt, Germany & 83.1826 \\
\hline $1 \mathrm{~mL} 26 \mathrm{G}$ syringes & BD Biosciences, US & 303176 \\
\hline $10 \mathrm{~cm}$ petri dish & Greiner Bio-One, Austria & 664160 \\
\hline $15 \mathrm{ml}$ Falcon tubes & Greiner Bio-One, Austria & 188271 \\
\hline $50 \mathrm{ml}$ Falcon tubes & Greiner Bio-One, Austria & 227261 \\
\hline 23G-butterfly & medi-tec, Germany & 4056353 \\
\hline 6-well plates & Corning, US & 3516 \\
\hline 96-well plates & Corning, US & 3594 \\
\hline 96-well plates & VWR, US & $732-2879$ \\
\hline 384-well plate & VWR, US & $732-3237$ \\
\hline \multicolumn{3}{|l|}{ Special consumables } \\
\hline $\begin{array}{l}\text { 384-well plates, ready-to- } \\
\text { use, prepared with barcoded } \\
\text { primers, and lysis solution }\end{array}$ & $\begin{array}{l}\text { Single Cell Discoveries, } \\
\text { Netherlands, } \\
\text { Muraro et al., } 2016\end{array}$ & $\mathrm{~N} / \mathrm{A}$ \\
\hline C-Tubes & Miltenyi, Germany & $130-093-237$ \\
\hline
\end{tabular}




\begin{tabular}{|c|c|c|}
\hline CryoMold & $\begin{array}{l}\text { Sakura, Tissue-Tek, } \\
\text { bought from VWR }\end{array}$ & 4557 \\
\hline EDTA tubes (Microvette) & Sarstedt, Germany & 20.1288 \\
\hline $\begin{array}{l}\text { FACS tube with } \\
\text { cell strainer cap }\end{array}$ & Corning, US & 352235 \\
\hline Filter bottle for FACS buffer & VWR, US & $10040-438$ \\
\hline Insert for six-well plate & VWR, US & $734-0032$ \\
\hline LS Columns & \begin{tabular}{|l|} 
Miltenyi, Germany \\
\end{tabular} & $130-042-401$ \\
\hline $\begin{array}{l}\text { Neubauer Counting } \\
\text { chamber }\end{array}$ & $\begin{array}{l}\text { KOVA Glasstic, Kova } \\
\text { International, US }\end{array}$ & 87144E \\
\hline Razor blades for vibratome & $\begin{array}{l}\text { commercial } \\
\text { (e.g. Wilkinson) }\end{array}$ & $\mathrm{N} / \mathrm{A}$ \\
\hline Pap pen for Immunostaining & Sigma Aldrich, US & Z672548-1EA \\
\hline Teflon bags for BMDMs & OriGen Biomedical, US & PL30-2G \\
\hline Reagent or resource & Source & Identifier \\
\hline \multicolumn{3}{|l|}{ Software } \\
\hline Aperio eSlide Manager & Leica, Germany & v.12.4.0.5043 \\
\hline CQ1 Software & Yokogawa, Japan & N/A \\
\hline FACS DIVA & BD Biosciences, US & v. 8 \\
\hline FlowJo & FlowJo LLC., US & v.10.4.2 \\
\hline GraphPad Prism & GraphPad Software & v.8.3.0 \\
\hline ITK-Snap & Yushkevich et al., 2019 & v.3.8.0 \\
\hline Microsoft Office for MAC & Microsoft, US & N/A \\
\hline Muriplan & X-Strahl Ltd., UK & N/A \\
\hline Murislice & X-Strahl Ltd., UK & N/A \\
\hline Paravision & Bruker, Germany & v.6.0.1 \\
\hline $\mathrm{R}$ & R Core Team, 2014 & v.3.4.3 \\
\hline RStudio & RStudio, US & v.1.1.453 \\
\hline \multicolumn{3}{|l|}{ Packages for R/RStudio } \\
\hline biomaRt & Durinck et al., 2009 & v.2.40.5 \\
\hline clusterprofiler & Yu et al., 2012 & v.3.12.0 \\
\hline DESeq2 & Love et al., 2014 & v.1.18.1 \\
\hline dplyr & Wickham et al., 2017 & v.1.0.2 \\
\hline enrichplot & Yu, 2020 & v.1.4.0. \\
\hline eulerR & Larsson, 2020 & v.6.1.0 \\
\hline ggfortify & Tang et al., 2016 & v.0.4.11 \\
\hline ggplot2 & Wickham, 2016 & v.3.3.2 \\
\hline ggrepel & Slowikowski, 2020 & v.0.8.2 \\
\hline gplots & Warnes et al., 2020 & v.3.1.0 \\
\hline pheatmap & Kolde, 2019 & v.1.0.12 \\
\hline RColorBrewer & Neuwirth, 2014 & v.1.1-2 \\
\hline reshape2 & Wickham, 2007 & v.1.4.4 \\
\hline $\mathrm{rgl}$ & Adler et al., 2020 & v. 0.100 .54 \\
\hline stats & R Core Team, 2019 & v.3.6.1 \\
\hline$x \mid s x$ & Dragulescu and Arendt, 2020 & v.0.6.4.2 \\
\hline \multicolumn{3}{|l|}{ Webpages } \\
\hline $\begin{array}{l}\text { EvalRSeq } \\
\text { HUSAR access via intranet }\end{array}$ & DKFZ & $\begin{array}{l}\text { https://www.dkfz.de/gpcf/root/men } \\
\text { u-left/analysis-pipelines/evalrseq } \\
\text { https://www.dkfz.de/gpcf/husar/ } \\
\text { access }\end{array}$ \\
\hline GEO NCBI & NCBI & https://www.ncbi.nlm.nih.gov/geo/ \\
\hline Heatmapper & Babicki et al., 2016 & http://www.heatmapper.ca \\
\hline $\begin{array}{l}\text { Human BrM } \\
\text { RNA-Seq data }\end{array}$ & Klemm et al., 2020 & $\begin{array}{l}\text { https://joycelab.shinyapps.io/ } \\
\text { braintime/ }\end{array}$ \\
\hline Metascape & Zhou et al., 2019 & www.metascape.org \\
\hline Venny 2.1 & Oliveros 2007-2015 & $\begin{array}{l}\text { http://bioinfogp.cnb.csic.es/ } \\
\text { tools/venny/index.html }\end{array}$ \\
\hline
\end{tabular}




\begin{tabular}{|l|l|l|}
\hline Devices & Leica, Germany & N/A \\
\hline Aperio Scan Scope & BD Biosciences, US & N/A \\
\hline BD FACS Aria Fusion & BD Biosciences, US & 647800 \\
\hline BD LSR Fortessa & Vacuubrand, Germany & 727000 \\
\hline BVC pumps & Yokogawa, Japan & N/A \\
\hline CQ1 microscope & Thermo Fisher Scientific, US & 75003060 \\
\hline Fresco 21 multicentrifuge & Miltenyi, Germany & $130-095-937$ \\
\hline $\begin{array}{l}\text { gentleMACS } \\
\text { Octo Dissociator }\end{array}$ & Thermo Fisher Scientific, US & 51026333 \\
\hline HERAcell 240i (incubator) & Heraeus, Germany & $3600-100-19$ \\
\hline Herasafe HS12 (flow) & ibidi, Germany & 11922 \\
\hline ibidi gas mixer & Caliper Lifescience, US & 123302 \\
\hline Ivis Lumina II & Thermo Fisher Scientific, US & $75002425 /-4503$ \\
\hline Megafuge (1.0R and 40R) & Kent Scientific, US & $13-005-204$ \\
\hline Mouse Surgical Tool Kit & Thermo Fisher Scientific & NanoDrop-1000 \\
\hline NanoDrop & Eppendorf, Germany & \\
\hline Pipettes (diff. sizes) & VWR, US & $612-0926$ \\
\hline PipettBoy & Miltenyi, Germany & $130-091-051$ \\
\hline QuadroMacs Magnet & PharmaScan, Bruker & N/A \\
\hline Small Animal MR Scanner & X-Strahl Ltd., UK & N/A \\
\hline $\begin{array}{l}\text { Small Animal Radiation } \\
\text { Research Platform (SARRP) }\end{array}$ & Thermo Fisher Scientific, US & ProFlex System, 4484073 \\
\hline Thermal Cycler & Leica, Germany & VT1200S \\
\hline Vibratome & Applied Biosystems & 4453537 \\
\hline ViiA7 qPCR system & Scientific Industry, US & SI-0256 \\
\hline Vortex-Genie 2 & Logos Biosystem, & C30001 \\
\hline $\begin{array}{l}\text { X-Clarity tissue clearing } \\
\text { system II }\end{array}$ & South Korea & \\
\hline
\end{tabular}

A) Cell lines and primary cells

All cells were maintained under humidified standard conditions at $37{ }^{\circ} \mathrm{C}$, and $5 \%$ $\mathrm{CO}_{2}$. Cells were regularly tested for mycoplasma. Passaging was performed according to standard protocols, and quantification for assays or injection was performed with manual counting chambers (Neubauer Counting).

\section{Tumor cell lines}

a) Maintenance and seeding for generation of supernatant

For this thesis, a murine and two human tumor cell lines have been used (see resource-table 1 , cell line overview table 2 ). Passaging was performed with trypsin upon PBS $(1 \mathrm{x})$ wash, and a standard centrifugation procedure with 5 min at 1500 rpm (= $491 \mathrm{~g})$. For long-term maintenance, cells from early passages were frozen within their respective standard media plus $10 \%$ DMSO, and kept in isopropanolcontainers $\mathrm{o} / \mathrm{n}$ with successive transfer into $-80{ }^{\circ} \mathrm{C}$, and further storage within liquid nitrogen tanks. 
Table 2.: Overview of tumor cell lines

\begin{tabular}{|c|c|c|c|}
\hline species & cell lines & cultivation in & media components \\
\hline murine & $\begin{array}{l}\text { 99LN } \\
\text { (different BrM variants) } \\
\text { (breast) }\end{array}$ & $\begin{array}{l}\text { complete DMEM } \\
\text { (advanced } \\
\text { DMEM upon } \\
\text { thawing) }\end{array}$ & $\begin{array}{l}\text { DMEM/Advanced DMEM } \\
+10 \% \text { FBS } \\
+1 \% \text { L-glutamine } \\
+1 \% \text { penicillin/streptomycin }\end{array}$ \\
\hline \multirow[t]{2}{*}{ human } & $\begin{array}{l}\text { H1_DL2 (melanoma) } \\
\text { (labeled with triple-imaging } \\
\text { vector: } \\
\text { TK-GFP-luc, TGL) }\end{array}$ & ALT DMEM & $\begin{array}{ll}\text { DMEM } & (450 \mathrm{ml}) \\
+10 \% \text { FBS } & (50 \mathrm{ml}) \\
+2 \% \text { L-glutamine } & (10 \mathrm{ml}) \\
+2 \% \text { penicillin/streptomycin } & (10 \mathrm{ml}) \\
\text { non-essential amino acids } & (16 \mathrm{ml}) \\
\text { plasmocin }(25 \mathrm{mg} / \mathrm{ml}) & (100 \mu \mathrm{l})\end{array}$ \\
\hline & $\begin{array}{l}\text { H2030 } \\
\text { (lung) } \\
\text { (labeled with triple-imaging } \\
\text { vector: } \\
\text { TK-GFP-luc, TGL) }\end{array}$ & complete RPMI & $\begin{array}{l}\text { RPMI } \\
+10 \% \text { FBS } \\
+1 \% \text { L-glutamine } \\
+1 \% \text { penicillin/streptomycin }\end{array}$ \\
\hline
\end{tabular}

For the generation of tumor cell supernatant (SN), $5 \times 10^{6}$ tumor cells were seeded into a $10 \mathrm{~cm}$ petri dish. Following $\mathrm{o} / \mathrm{n}$ incubation, the media was exchanged with FBS-free media. This conditioned medium was harvested $24 \mathrm{~h}$ later and centrifuged for $5 \mathrm{~min}$ at $1500 \mathrm{rpm}$. The tumor cell SN was used for stimulation of BMDMs or microglia.

b) Viral transduction

In order to stably transduce tumor cells with fluorescent reporter constructs, spinfection was performed. Therefore, $1 \times 10^{5}$ tumor cells were seeded into multiple wells of a 24-well plate, and incubated o/n. Supernatant containing the lentiviral vector (pHR'-SBW) was titrated in different concentrations within $500 \mu \mathrm{l}$ medium/per well. Consecutively, plates were centrifuged for $1 \mathrm{~h}$ at $32{ }^{\circ} \mathrm{C}$ and $872 \mathrm{~g}$. Spinfected cell-containing plates were incubated again for $37^{\circ} \mathrm{C}$, and medium was changed after $7 \mathrm{~h}$. Once confluent again, cells were harvested and transferred into standard cell culture flasks, passaged for 2-3 more times, and sorted via FACS (BD Aria Fusion) for high BFP-positive cells under sterile conditions. This stably transduced cells (99LN-BFP) were expanded, and subsequently used for ex vivo brain slice experiments, to ensure long-term fluorescent signals of tumor cells. 
2. Microglia cell line

For some stimulation experiments, a murine microglia cell line was used (table 3), which was kept under standard conditions (see above). However, media + cytokines were changed every 2 - 3 days, and EOC2 cells were passaged via manually scratching with a cell scraper.

Table 3.: Overview of microglia cell line

\begin{tabular}{|l|l|l|l|}
\hline species & cell line & cultivation in & medium components \\
\hline murine & EOC2 & complete DMEM + & DMEM/ \\
& & cytokines & $+10 \% \mathrm{FBS}$ \\
& & $(2 \mathrm{ng} / \mathrm{ml} \mathrm{rmIL-34,}$ & $+2 \%$ L-glutamine \\
& & $0.2 \mathrm{ng} / \mathrm{ml} \mathrm{rmTGF}-ß)$ & $+1 \%$ penicillin/streptomycin \\
\hline
\end{tabular}

\section{Primary cells}

a) Bone marrow-derived macrophages (BMDM)

Primary cell isolation was performed from WT BL/6 mice as previously described (Quail et al., 2016). Female mice were anesthetized (see below), and tibia and femur of both hind limbs dissected under sterile conditions and transferred into cold HBSS. The remaining tissue attached to bones was carefully removed, and bone marrow flushed out with a fine cannula (approx. 28 - 30G) and 5 - $10 \mathrm{ml}$ complete DMEM into a $50 \mathrm{ml}$ tube, topped with a $70 \mu \mathrm{m}$ cell strainer. Flush-outs of one mouse were pooled and upon centrifugation $(491 \mathrm{~g}, 5 \mathrm{~min}$ ) cell pellets were resuspended in $30 \mathrm{ml}$ complete DMEM, supplemented with $30 \mu \mathrm{l}$ M-CSF1 (final concentration of $1 \mathrm{ng} / \mathrm{ml}$, diluted in PBS + $0.1 \%$ BSA). Differentiation of bone marrow progenitor cells was performed in special Teflon bags under standard conditions for seven days. Importantly, every other day cells were harvested, centrifuged, and resuspended in fresh media plus M-CSF1 (new media plus cytokine at: d0, d2, d4, d6).

b) Transient labeling

For fluorescent visualization of BMDMs in ex vivo brain slice settings, cells were harvested on day 6 of differentiation, centrifuged, and BMDM cell pellets resuspended in FBS-free media supplemented with fluorescent cell tracker diluted in sterile DMSO, according to manufacturer's instructions. Cells were incubated in 15 $\mathrm{ml}$ conical tubes at $4{ }^{\circ} \mathrm{C}$, before another round of centrifugation. Finally, cell tracker- 
labelled BMDMs were transferred back into Teflon bags and incubated $\mathrm{o} / \mathrm{n}$ in complete media + cytokine until further usage.

\section{Stimulation of BMDMs and MG}

To generate activation and phenotypic profiles, BMDM or MG were stimulated with SN from tumor cells. Cells were seeded into six-well plates (BMDMs: $2 \times 10^{6}$ cells/well, EOC: $1 \times 10^{6}$ cells/well) and incubated o/n. For the MG samples, all conditions contained cytokines. Adhered cells were washed and the media exchanged with tumor cell SN, followed by further incubation for $24 \mathrm{~h}$. As control, FBS-free medium was used. Afterwards, cells were rinsed with PBS, any remaining liquids were removed and cells lysed and resuspended in $1 \mathrm{ml}$ TRIzol. Cell lysates within TRIzol were transferred into Eppendorf tubes, and stored at $-80{ }^{\circ} \mathrm{C}$ until further downstream analyses.

B) Mouse models and in vivo methods

\section{Mouse strains}

For BrM experiments, several mouse strains were used. All animals were kept under standard, SPF-free conditions, with sexes separated and a maximum of 5 individuals per cage (individually ventilated). Food and water was provided ad libitum. Sometimes in-house bred Balb/c nude mice (both sexes) were used for experiments involving $\mathrm{H} 2030$. For that, mice were obtained by breeding heterozygous females (with fur) with homozygous males (nude), and genotypes determined based on their phenotype (nude vs. with fur).

For tissue harvest (BMDM generation, control brains), BL6 mice were used. For ex vivo brain slice experiments, brains of transgenic in-house bred CX3CR1-GFP/wt mice were used. Furthermore, tissue was harvested from in-house breeding of GFAP-Cre x Rosa26_tdTomato mice (see table 1).

Genotyping was performed from ear biopsies with Transnetyx (US).

All animal experiments were approved by the government committee (Regierungspräsidium Darmstadt, Germany) and were in accordance with the requirements of the German Animal Welfare Act. 


\section{BrM modelling}

To model BrM in vivo, mice were anesthetized with $100 \mu \mathrm{l} / 10 \mathrm{~g}$ body weight ketaminxylazin solution (250 mg/kg ketamine and $25 \mathrm{mg} / \mathrm{kg}$ xylazin), and intra-cardially

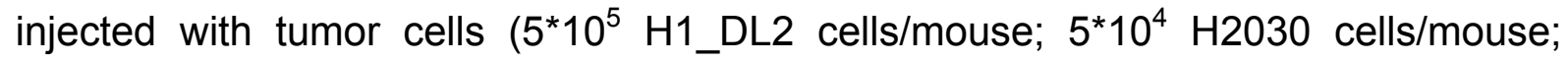
$1 * 10^{5}$ 99LN-BrM2 cells/mouse) in $200 \mu$ sterile PBS. Specifically, xenograft BrM models were induced within Balb/c nude mice (cell lines: H1_DL2 and H2030) injected with 6-8 weeks of age, whereas the syngeneic breast-to-brain metastasis model was induced with female WT BI6 mice (see table 1), injected starting at 10 - 12 weeks of age. The respective official protocol number is F123/1016. Experimental mice were closely monitored, and weighted once per week until final experiments.

\section{Tracking of tumor progression}

The following methods were applied to closely examine tumor progression, starting at 3 (H1_DL2 and H2030), or at 5 weeks following injection (syngeneic model, 99LNBrM).

\section{a) Bioluminescent imaging}

Xenograft models were imaged via bioluminescent imaging (BLI, via IVIS), following subcutaneous injection of $100 \mu \mathrm{l}$ D-luciferin $\left(15 \mathrm{mg} / \mathrm{ml}\right.$ in $\left.\mathrm{dH}_{2} \mathrm{O}\right)$. During measurements, mice were constantly held anesthetized with $2 \%$ isoflurane for a total duration of about 10 - 15 min. Radiance values were used to determine BrM induction and progression, as defined by photons $/ \mathrm{sec} / \mathrm{cm}^{2} / \mathrm{sr}$. These values were further used to stratify mice (e.g. small vs. large BrM, mice for WBRT).

\section{b) MRT measurement}

Tumor progression and final imaging of syngeneic BrM models was performed via magnet resonance imaging (MRI), as previously described (Chae et al., 2019). Tijna Alekseeva, Woony Chae, and/or Marco Lolies performed MR measurements of mice used in this thesis. Briefly, mice were injected intra-peritoneally (i.p.) with $100 \mu \mathrm{l}$ contrast agent (Gadubutrol), and MRI measurements were performed on anesthetized (2\% isoflurane) mice with a 7 Tesla Small Animal MR Scanner. Data acquisition was performed with the Paravision software of mice in coronal orientation, and T1- and T2-weighted sequences obtained. Volumetric analysis of BrM lesions was performed with DICOM files using the segmentation tool in ITK-Snap software. 


\section{Whole brain irradiation of mice}

In order to mimic standard of care therapy of BrM, we developed a protocol to treat mice with (hypo-) fractionated whole-brain ionizing radiation/radiotherapy (WBRT), as previously described (Chae et al., 2019, Schulz et al., 2020).

CT imaging and WBRT were performed with the Small Animal Radiation Research Platform (SARRP) in collaboration with the group of Prof. Dr. Franz Rödel (KGU). BrM-bearing or control mice were anesthetized ( $2 \%$ isoflurane), stabilized in a prone position and CT imaging was performed in Murislice, with a cone beam CT (CBCT) operating at $60 \mathrm{kV}$ and $0.8 \mathrm{~mA}$, which is integrated within the SARRP. CT images were loaded into the treatment planning software Muriplan, and based on highly precise images, contouring, dose calculation, isocenter defining and treatments were performed (figure 3). Finally, WBRT was applied fractionated for five consecutive days with $2 \mathrm{~Gy}$, or as a single dose of $10 \mathrm{~Gy}$ (= hypo-fractionated) using a $10 \times 10$ $\mathrm{mm}$ collimator in an Arc, operating with $220 \mathrm{kV}$ and $13 \mathrm{~mA}$, yielding $5.2 \mathrm{cGy} / \mathrm{sec}$.

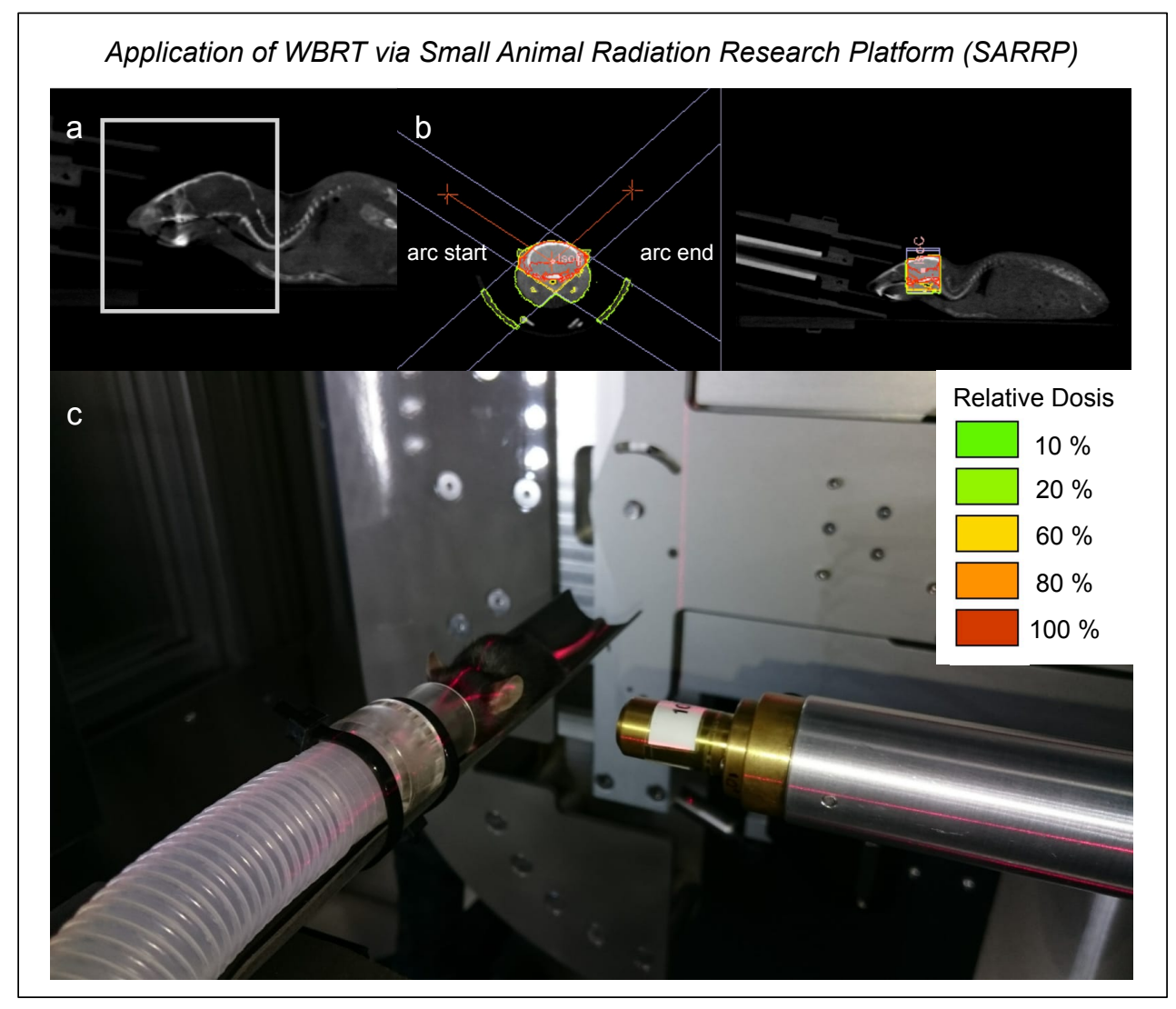

Figure 3: Whole brain radiotherapy of mice with the SARRP. a) Head region of interest as seen in CT image. b) Definition and contouring of treatment regimen. c) Representative WBRT set-up, here with anesthetized BI6 mouse. 


\section{Final isolation of brain/BrM tissue and peripheral blood}

For further downstream analyses at defined or experimental endpoints, mice were anesthetized (ketamin/xylazin), and dissected under sterile conditions. Peripheral blood of control mice for FACS was obtained by cardiac puncture, and blood transferred and stored in EDTA-coated tubes (Microvette) on a shaker at RT until further processing.

Control or (irradiated) BrM-bearing brains were isolated from anesthetized mice. For all histologic samples, full brains were isolated upon trans-cardial perfusion of mice with PBS (plus PFA in some cases), and brains were stored in PFA (Histofix).

All flow cytometry (FCM) and FACS, samples were obtained from PBS-perfused mice. As controls for FCM and FACS, as well as for brain slice experiments, full brains were isolated, as it was done for FCM of all 99LN-samples. Every other FCM and FACS analyses were performed on macro-dissected tissue. This was done by manually macro-dissecting the brain tissue with the help of a binocular stereomicroscope. H1_DL2 and H2030 BrMs were macro-dissected based on BLI signal, upon final imaging of mice. BrMs from 99LN mice were dissected based on final MR images. All tissues or full brains were stored in cold HBSS until further processing.

C) Brain slice (BrSI) assay and live cell imaging

Since methods to study cellular interaction in an "in vivo like" setting are rather rare, an advanced multicellular ex vivo brain slice assay was developed during this thesis (manuscript in preparation), which allows the observation and determination of cellular behavior via live cell imaging. The basic protocol was derived from organotypic slice assays performed similar as described in Valiente et al. (2014).

\section{Slice generation}

Brain slices were generated with a vibratome at $0.5 \mathrm{~mm} / \mathrm{s}$, by gently cutting 200 $350 \mu \mathrm{m}$ thick slices in transversal or coronal orientation within cold HBSS. For fixed sections, tissue was cut within normal PBS. The (living) slices were carefully transferred into sterile tissue culture inserts within 6-well plates and about $2-3 \mathrm{ml}$ brain slice medium (table 4) applied. BrSI were incubated for several $\mathrm{h}$ till o/n at 37 ${ }^{\circ} \mathrm{C}$ and $5 \% \mathrm{CO}_{2}$. Importantly, medium was changed once per day. 
Table 4: Components of brain slice medium

\begin{tabular}{|l|l|}
\hline reagent & amount (for 500 $\mathbf{~ m l})$ \\
\hline MEM medium & $250 \mathrm{ml}(50 \%)$ \\
\hline HBSS & $125 \mathrm{ml}(25 \%)$ \\
\hline Normal Horse Serum & $125 \mathrm{ml}(25 \%)$ \\
\hline L-glutamine & $0.5 \mathrm{ml}(0.2 \mathrm{mM})$ \\
\hline penicillin-streptomycin & $0.5 \mathrm{ml}(100 \mathrm{U} / \mathrm{ml}-100 \mu \mathrm{gg} / \mathrm{ml})$ \\
\hline D-glucose & $2.25 \mathrm{~g}(4.5 \mathrm{mg} / \mathrm{ml})$ \\
\hline
\end{tabular}

\section{Multi-cellular culture}

For the set-up of multi-cellular BrSI culture, a sequential protocol was developed. Upon incubation of freshly cut BrSI, various amounts of tumor cells (99LN-BFP) were applied within $1 \mu \mathrm{l}$ medium as a small drop on top of the $\mathrm{BrSl}$ inside the insert, which however were not covered with medium. Upon trying different quantities, an amount of $10^{5}$ tumor cells was used. Importantly, upon application of cells, it was strictly avoided to touch/move plates again, since every vibration resulted in loss of tumor cells on top of the BrSl. Following an incubation for about 2 - $3 \mathrm{~h}$, another cell type (here: BMDMs) was applied at approximal the same location as tumor cells were added. Here, about $5 \times 10^{3}$ cell tracker-labeled BMDMs were applied, and the whole set-up incubated for another 2 - $3 \mathrm{~h}$ at RT, before continuing imaging/fixation. Besides "immediate" usage for imaging, multi-cellular cultures were further incubated for several days to allow growth (of e.g. tumor cells) and interaction between different cell types. Media was changed once per day, and slices finally used for imaging or fixation.

\section{Imaging and analysis}

For immediate imaging upon multi-cellular culture set-up, 6-well plates were transferred into the confocal microscope CQ1. This microscope was equipped with a gas mixer (ibidi), which delivered $5 \% \mathrm{CO}_{2}$ via constant air pressure into the humidified sample chamber of the CQ1 at $37^{\circ} \mathrm{C}$, therefore allowing incubation under standard conditions.

Following the set-up of samples and definition of acquisition field, confocal live cell imaging was performed, typically in a range of $10-50 \mu \mathrm{m}$ ( $\mathrm{z}$ area), every $10-15$ min, and in total for $24 \mathrm{~h}$. For live cell image acquisition, a $10 \times$ or $20 \times$ objective was used. The analysis and movie generation was done with the CQ1 software, and snapshots of certain time points used for visualization in here. 
Note that this approach offers high flexibility regarding the number of samples, time points, etc. BrSI before and upon imaging furthermore can be/were fixed for subsequent immuno-fluorescence staining.

D) Histology on thin and thick mouse brain sections

All primary and secondary antibodies used for immuno-fluorescence or immunohistochemistry can be found in table 5 .

\section{HE-staining and immuno-histochemistry}

Brains for HE-staining and/or immuno-histochemistry were stored in Histofix for several days, followed by embedding in paraffin. HE-stainings were performed with the automated staining device Leica Autostainer XL according to standard protocols. Immuno-histochemistry was performed on a semi-automated platform (Leica Bond Max), including deparaffinization, rehydration, citrate buffer-based antigen retrieval, and blocking of unspecific binding of proteins and peroxidase, which was followed by primary antibody incubation. Thereafter, HRP-labeled secondary antibody incubation was applied, followed by DAB conversion. Notably, all of the above-mentioned steps were performed at the Histology Core Facility of the Georg-Speyer-Haus.

\section{Immunofluorescence of thin brain sections}

In order to perform immunofluorescence staining, thin brain sections were prepared from full brains as well. PFA-fixed brains were transferred into $30 \%$ sucrose until full equilibration (several days). Brains were cut manually and embedded into cryo-molds with O.C.T. compound. Samples were equilibrated at RT and successively transferred to $-80^{\circ} \mathrm{C}$, and stored until further usage. Cutting was performed at the Histology Core Facility of the Georg-Speyer-Haus, and $5-10 \mu \mathrm{m}$ slides generated on super-frost glass slides, which were stored at $-80^{\circ} \mathrm{C}$.

For staining, slides were dried at RT for several $h$, before a pap pen was used to encircle sections, followed by rehydration with PBS $(3 \times 10 \mathrm{~min})$. Slides were blocked and permeabilized with $3 \%$ BSA in PBS plus $0.1 \%$ TritonX-100 for $1 \mathrm{~h}$ at RT in a humidified chamber, followed by incubation with primary antibodies in $1.5 \%$ BSA in PBS for $2 \mathrm{~h}$ at $\mathrm{RT}$, or o/n at $4{ }^{\circ} \mathrm{C}$. Upon PBS washing $(3 \times 10 \mathrm{~min})$, samples were incubated with secondary antibodies in $1.5 \%$ BSA in PBS (all diluted 1:500) for 45 min at RT, followed by rinsing in PBS. Finally, samples were counterstained with 
DAPI (1:2500 in PBS) for 5 min at RT, before mounting with DAKO and coverslips. Slides were dried at RT, and stored at $4{ }^{\circ} \mathrm{C}$ until imaging.

\section{Immunofluorescence of thick brain sections}

a) Slice generation and tissue clearing

Thick tissue sections of PFA-stored brains were derived similarly as described for $\mathrm{BrSl}$ (see C.1, BrSI generation), and sections were cut with a vibratome (200 - 350 $\mu \mathrm{m})$. Slices were stored in PBS at $4{ }^{\circ} \mathrm{C}$, and cleared before staining. Sections were processed in a tissue clearing system (X-Clarity), and infused with a SDS-containing solution, temperature-controlled electrophoresis for 2 - $3 \mathrm{~h}$ at $0.6 \mathrm{~A}$. For IF staining, single slices were transferred into 24-well plates and every subsequent steps were performed in it, in order to minimize required volume.

\section{b) Blocking and staining}

Following standard procedures, slices were washed again in PBS ( $2 \times 10 \mathrm{~min})$, and incubated in $3 \%$ BSA in PBS $+0.1 \%$ Triton X-100 for blocking of unspecific antibody binding and permeabilization $3-5 \mathrm{~h}$ at RT. Afterwards, slices were incubated with primary antibodies at RT for 12 - $24 \mathrm{~h}$. Upon brief washing ( $3 \times 10 \mathrm{~min}$ ) in PBS, samples were now incubated with secondary antibodies (all 1:500) for $3-5 \mathrm{~h}$, and both primaries and secondaries were diluted in $1.5 \%$ BSA in PBS. Following a brief PBS rinse, samples (if applicable) were counterstained with DAPI (see above) for 20 - 60 min at RT, before slices were washed again three times with PBS for 10 min each. Finally, slices were transferred on glass slides and mounted with X-Clarity mounting medium and cover slips. Samples were dried at RT, and stored at $4{ }^{\circ} \mathrm{C}$ until imaging.

\section{c) Confocal imaging}

To generate high quality confocal images from IF-stained brain slices, the CQ1 was used (see C.3, imaging and analysis). Images were taken from a z-stack typically between 20 - $50 \mu \mathrm{m}$, and with a 10x, 20x, and 60x objective. Processing of images was performed with the CQ1 software, and MIP (maximum intensity projection) pictures taken for representation. 
Table 5: Primary and secondary antibodies for histology

\begin{tabular}{|c|c|c|c|}
\hline antigen & $\begin{array}{l}\text { host and clonality } \\
\text { monoclonal (monocl.), } \\
\text { polyclonal (polycl.) }\end{array}$ & dilution & source and identifier \\
\hline \multicolumn{4}{|c|}{$\begin{array}{l}\text { primary } \\
\text { (anti-mouse/cross-reactive) }\end{array}$} \\
\hline B220 & $\begin{array}{l}\text { rat, monocl., } \\
\text { RA3-6B2 }\end{array}$ & $1: 2000$ & $\begin{array}{l}\text { Novus Biologicals, US } \\
\text { NB100-77420SS }\end{array}$ \\
\hline $\mathrm{C} 1 \mathrm{Q}$ & rat, monocl., $7 \mathrm{H} 8$ & $1: 100$ & Abcam, UK, ab11861 \\
\hline C3/C3a & chicken, polycl. & $1: 200$ & Abcam, UK, ab48581 \\
\hline C3AR1 & rat, monocl., 14D4 & $1: 1000$ & $\begin{array}{l}\text { HyCult Biotech, } \\
\text { Netherlands, HM1123 }\end{array}$ \\
\hline CD3 & rabbit, polycl. & undiluted & Agilent, US, IS503 \\
\hline CD19 & rat, monocl. & $1: 1000$ & $\begin{array}{l}\text { eBioscience, US } \\
14-0193-85\end{array}$ \\
\hline CD206 /MMR & goat, polycl. & $1: 1000$ & $\begin{array}{l}\text { R\&D Systems, US } \\
\text { AF2535 }\end{array}$ \\
\hline GFAP & goat, polycl. & $1: 1000$ & Abcam, UK, ab53554 \\
\hline GFP & chicken, polycl. & $1: 1000$ & Abcam, Uk, ab13970 \\
\hline IBA1 & rabbit, polycl. & $1: 1000$ & $\begin{array}{l}\text { Wako Chemicals, Japan } \\
019-19741\end{array}$ \\
\hline NEUN & $\begin{array}{l}\text { rabbit, monocl., } \\
\text { EPR12763 }\end{array}$ & $1: 10.000$ & Abcam, UK, ab177487 \\
\hline TMEM119 & guinea pig, polycl. & $1: 1000$ & SySy, Germany, 400004 \\
\hline \multicolumn{4}{|c|}{ secondary (+fluorochrome) } \\
\hline anti- & polyclonal & \multirow{17}{*}{$1: 500$} & $\begin{array}{l}\text { Jackson } \\
\text { ImmunoResearch, US }\end{array}$ \\
\hline chicken (Alexa488) & donkey & & $703-545-155$ \\
\hline chicken (Alexa594) & donkey & & $703-585-155$ \\
\hline chicken (Alexa647) & donkey & & $703-606-155$ \\
\hline goat (Alexa488) & donkey & & $705-546-147$ \\
\hline goat (Alexa594) & donkey & & $705-586-147$ \\
\hline goat (Alexa647) & bovine & & $805-605-180$ \\
\hline guinea pig (Alexa488) & donkey & & $706-545-148$ \\
\hline guinea pig (Alexa594) & donkey & & $706-585-148$ \\
\hline guinea pig (Alexa647) & donkey & & $706-605-148$ \\
\hline rabbit (DyLight 405) & donkey & & $111-475-003$ \\
\hline rabbit (Alexa488) & donkey & & $705-546-147$ \\
\hline rabbit (Alexa594) & donkey & & $711-586-152$ \\
\hline rabbit (Alexa647) & donkey & & $711-605-152$ \\
\hline rat (Alexa488) & donkey & & $712-546-150$ \\
\hline rat (Alexa594) & donkey & & $712-586-150$ \\
\hline rat (Alexa647) & donkey & & $712-605-150$ \\
\hline
\end{tabular}

E) Flow cytometry and FACS

\section{Preparation of peripheral blood}

Peripheral blood was transferred from EDTA tubes into FCM round-bottom tubes with $1 \mathrm{ml}$ of lysis buffer (red blood cell lysis, $1 \mathrm{x}$ ), following addition of another $1 \mathrm{ml}$ of lysis buffer. Samples were incubated for 10 - 15 min at RT, followed by a washing step with $2 \mathrm{ml}$ degassed FACS buffer (1 x PBS with $2 \%$ FBS) and centrifugation for 5 min 
at $2000 \mathrm{~g}$. Supernatant was discarded and the pellet resuspended in fresh $1 \mathrm{ml}$ lysis buffer, and the whole lysis-wash procedure repeated 3 - 4 times. Upon lysis of most of the erythrocytes (samples become almost transparent) cell pellets were resuspended in Fc block (1:1000 in FACS buffer) following the last wash, and incubated for $10-15 \min$ at $4{ }^{\circ} \mathrm{C}$. Subsequently, samples were stained with antibody mix (table 6) in $100 \mu$ FACS buffer for $15-30$ min at $4{ }^{\circ} \mathrm{C}$, before another washing round. Finally, samples were resuspended in FACS buffer $+2.5 \mathrm{mM}$ EDTA and filtered through a cell strainer cap of a new FACS tube. Samples were kept on ice until FCM or FACS.

\section{Preparation of brain tissue}

Macro-dissected brain/BrM or full brain tissue was transferred into C-tubes containing digestion mix (per sample: $3890 \mu$ l buffer $X+40 \mu$ l buffer $Y+20 \mu$ l enzyme $A+50 \mu l$ enzyme $U$ ) and tissue was enzymatically digested into a single cell suspension using the Brain Tumor Dissociation Kit according to manufacturer's instructions and the GentleMacs Octo Dissociator (program running approx. $20 \mathrm{~min}$ ). Note however, since it was observed that the presence of certain antigens (especially of T cells) seemed to be influenced via the recommended enzyme mixture, 99LN-BrM tissue for FACSort was digested with only half the amount of enzyme $A$ and $U$. Upon finishing the digestion, single cell suspensions were filtered through a $70 \mu \mathrm{m}$ cell strainer, washed with $10 \mathrm{ml}$ HBSS and centrifuged (10 mins, $300 \mathrm{~g}, 4{ }^{\circ} \mathrm{C}$ ). The $\mathrm{SN}$ was discarded, the cell pellet was resuspended in $2 \mathrm{ml}$ of Myelin Removal Beads mixture (diluted 1:10 in degassed FACS buffer) and incubated for 15 mins at $4{ }^{\circ} \mathrm{C}$. The incubation was stopped by adding $18 \mathrm{ml}$ FACS buffer, followed by centrifugation (s. above). The bead-coupled suspension was resuspended in $1 \mathrm{ml}$ of FACS-buffer and applied to pre-primed LS columns within the QuadroMacs Magnet. Columns were washed once with $2 \mathrm{ml}$ of FACS buffer, the flow-through was collected in $15 \mathrm{ml}$ conical tubes, and the suspension centrifuged (s. above). Based on the degree of remaining blood, samples were incubated once with RBC lysis buffer (s. above) in order to remove remaining erythrocytes, if necessary. This was mainly performed for $\mathrm{H} 2030-\mathrm{BrMs}$, since this tissue usually contained a high blood content. Centrifugation was performed as before and cell pellets were washed once with $5 \mathrm{ml}$ of FACS buffer. Following another centrifugation, samples were splitted into FACS tubes with about $1-5 * 10^{6}$ cells $/ \mathrm{ml}$, and finally blocked with Fc-Block (1:1000 in $100 \mu \mathrm{l}$ of 
FACS-buffer) for 10 to 30 mins at $4{ }^{\circ} \mathrm{C}$. Right afterwards, samples were stained with antibody-mix containing eFluor-780 for live/dead-discrimination in another $100 \mu \mathrm{l}$ of FACS-buffer (table 6). Finally, samples were washed once with FACS-buffer + EDTA, centrifuged and the sample pellet resuspended in FACS-buffer + EDTA and filtered into another FCM tube. Samples were kept on ice until FCM/FACS.

\section{Flow cytometric analysis}

For overall analyses of various BrM samples, the following panels for FCM and FACS were applied, focusing on the myeloid and lymphoid compartment (table 6).

Table 6: Overview of FCM/FACS antibodies

\begin{tabular}{|c|c|c|c|c|}
\hline panel & $\begin{array}{l}\text { target/antigen } \\
\text { (+ fluorochrome) }\end{array}$ & host & $\begin{array}{l}\text { dilution for } 1 x \text { in } \\
100 \mu \text { I FACS buffer }\end{array}$ & source and identifier \\
\hline \multicolumn{5}{|c|}{ myeloid } \\
\hline & CD45 (A700) & rat & $1: 500$ & $\begin{array}{l}\text { Biolegend, US, } \\
\text { clone 30-F11, } 103128\end{array}$ \\
\hline & CD11b (BV605) & rat & $1: 1000$ & BD, US, clone M1-70, 563015 \\
\hline & Ly6C (PerCP-Cy5.5) & rat & $1: 250$ & $\begin{array}{l}\text { Biolegend, US, } \\
\text { clone HK1.4, } 128012\end{array}$ \\
\hline & Ly6G (BV421) & rat & $1: 500$ & $\mathrm{BD}, \mathrm{US}$, clone 1A8, 562737 \\
\hline & CD49d (Pe-Cy7) & rat & $1: 500$ & $\begin{array}{l}\text { Biolegend, US, } \\
\text { clone R1-2, } 103618\end{array}$ \\
\hline & $\begin{array}{l}\text { eFluor-780 } \\
\text { (diluted 1:10 in PBS) }\end{array}$ & N/A & $2 \mu \mathrm{l} / 100 \mu \mathrm{l}$ & $\begin{array}{l}\text { Thermo Fisher Scientific, US, } \\
65-0865-14\end{array}$ \\
\hline \multicolumn{5}{|c|}{ lymphoid } \\
\hline & CD45 (BV605) & rat & $1: 500$ & BD, US, clone 30-F11, 563053 \\
\hline & CD3 (PE) & $\begin{array}{l}\text { cell } \\
\text { line }\end{array}$ & $1: 500$ & $\begin{array}{l}\text { Miltenyi, Germany, } \\
\text { clone REA641, 130-120-826 }\end{array}$ \\
\hline & B220 (BV421) & rat & $1: 300$ & BD, US, clone RA3-6B2, 562922 \\
\hline & CD4 (PE-Vio770) & rat & $1: 500$ & $\begin{array}{l}\text { Miltenyi, Germany, } \\
\text { clone GK1.5, 130-124-712 }\end{array}$ \\
\hline & CD8 (PerCP-Cy5.5) & rat & $1: 500$ & BD, US, clone 53-6.7, 561109 \\
\hline & $\begin{array}{l}\text { eFluor-780 } \\
\text { (diluted 1:10 in PBS) }\end{array}$ & $\mathrm{N} / \mathrm{A}$ & $2 \mu \mathrm{l} / 100 \mu \mathrm{l}$ & $\begin{array}{l}\text { Thermo Fisher Scientific, US, } \\
65-0865-14\end{array}$ \\
\hline
\end{tabular}

In more detail, panels were designed and pre-tested according to good FCM practice. To determine populations of interests, FMO (final minus one) controls were used. Both panels (exemplified in figure 4) were similarly set up at BD LSR Fortessa (for FCM) and BD FACS Aria Fusion (for FCM and FACS), and devices were operated via BD Diva Software. FACS was exclusively performed with the Fusion, whereas the following data were acquired on both instruments. FCM data of $\mathrm{H} 2030$, and 99LN models were obtained with Fortessa, whereby the latter one was derived form full brain samples. Since relative cellular quantities of control brains (of Balb/c 
nude mice) were comparable between Fortessa and Fusion device, FCM data of H1_DL2 were derived via acquisition at the Fusion instrument, in order to compare between H1_DL2 and H2030 data.

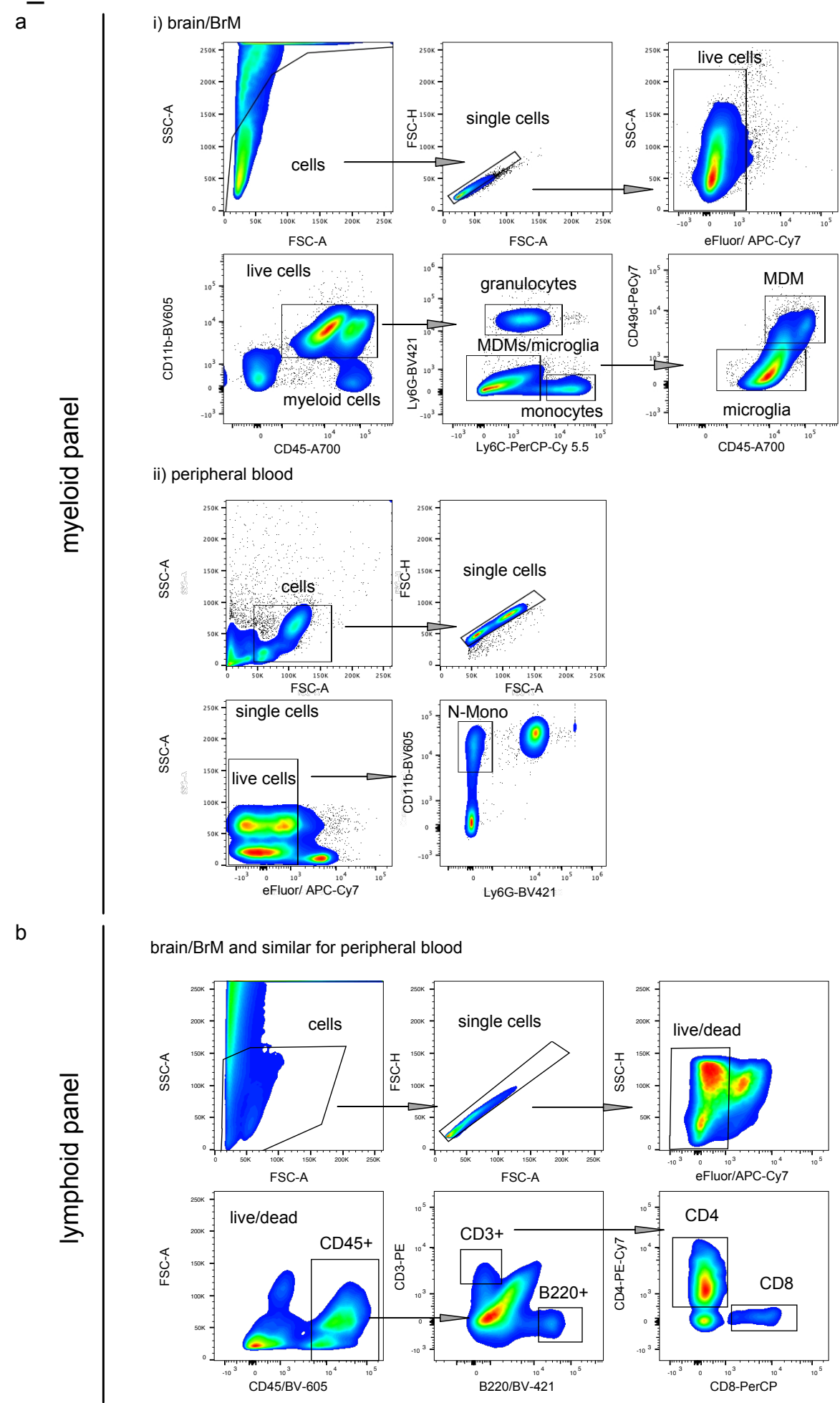

Figure 4: Overview of the gating strategies for FCM and FACS panels. The upper two panels correspond to the myeloid panel (a) used for FCM and FACS of brain/BrM and peripheral blood. Images are modified from Schulz et al., 2020. The lower compilation represents the lymphoid panel (b), where both, brain/BrM and peripheral blood cells were analyzed and sorted with the same gating strategy. 


\section{FACS of immune cells for IF, qPCR and RNA-Seq}

For molecular downstream analyses of the BrM-associated inflammatory microenvironment, distinct cell populations were sorted (BD FACS Aria Fusion). To confirm the lymphoid gating strategy, indicated cell types were sorted on BSA-coated ( $3 \%$ in PBS, incubated o/n at $37^{\circ} \mathrm{C}$ ) coverslips. Samples were fixed with PFA for 30 min at RT, followed by washing with PBS, and a consecutive staining similar as described above in the section IF on thin brain sections (see D.2, figure 15b).

For all bulk RNA-Seq experiments and qPCRs, cell populations of interest were sorted into cooled $1.5 \mathrm{ml}$ tubes, prepared with 50 - $100 \mu \mathrm{l}$ PBS. If the final sample volume exceeded $250 \mu \mathrm{l}$, tubes were briefly spun down $(5 \mathrm{~min}, 300 \mathrm{~g})$ and $\mathrm{SN}$ removed until $250 \mu \mathrm{l}$ remained, which was mostly the case with samples for qPCR, since those samples contained a higher amount of sorted cells. All samples were constantly cooled on ice, until $0.75 \mathrm{ml}$ TRIzol LS was added, and total samples were homogenized via pipetting. Samples were frozen on dry ice and/or immediately stored at $-80^{\circ} \mathrm{C}$.

For single cell RNA-Seq, single cells were sorted directly into ready-to-use 384 well cell capture plates (Single Cell Discoveries). Plates were immediately sealed again and stored at $-80^{\circ} \mathrm{C}$ until transfer and library preparation.

F) RNA isolation, cDNA synthesis and GPCR

\section{RNA isolation}

For qPCR experiments, RNA was isolated from sorted primary cells out of TRIzol LS, or stimulated MGs/BMDMs out of TRIzol according to manufacturer's instructions. Upon incubation and complete dissociation of the samples at RT (ca. 2 - 5 min), 0.2 $\mathrm{ml}$ chloroform was added per $1 \mathrm{ml}$ TRIzol/ $0.75 \mathrm{ml}$ TRIzol LS, samples were shaken for $15 \mathrm{sec}$, incubated at RT for 3 - $5 \mathrm{~min}$, and centrifuged for $15 \mathrm{~min}$ at $4{ }^{\circ} \mathrm{C}$ with $12.000 \mathrm{~g}$ to separate phases. The upper phase containing RNA was transferred into a new $1.5 \mathrm{ml}$ tube, and $0.5 \mathrm{ml} 100 \%$ isopropanol added, followed by addition of 1.5 $\mu \mathrm{l}$ glycoblue as co-precipitate. Samples were incubated for $10 \mathrm{~min}$ at RT, before centrifugation for $10 \mathrm{~min}$ at $4{ }^{\circ} \mathrm{C}$ with $12.000 \mathrm{~g}$. Now the supernatant was discarded carefully, the RNA pellet was washed with $1 \mathrm{ml} 75 \%$ ethanol (prepared with DEPCwater), samples were vortexed briefly and centrifuged for 5 min at $4{ }^{\circ} \mathrm{C}$ with $7.500 \mathrm{~g}$. Afterwards, the washing solution was discarded and RNA pellets air-dried for 
approximately $30 \mathrm{~min}$ at RT. Samples were resuspended in 20 - $50 \mu \mathrm{l}$ DEPC-water, and incubated at $60^{\circ} \mathrm{C}$ for $10-15 \mathrm{~min}$, before storing on ice, or long-term at $-80^{\circ} \mathrm{C}$. Before further downstream processing, sample quality and quantity were measured via NanoDrop spectrophotometer, and RNA concentrations equalized between conditions by addition of respective amount of DEPC-water, if necessary.

\section{2. cDNA synthesis}

RNA was reverse transcribed into cDNA using the High-Capacity cDNA reverse Transcription kit according to the manufacturer's instructions. Ten $\mu$ of RNA were mixed with $10 \mu \mathrm{l}$ of the $2 \mathrm{x}$ reverse transcription mix, containing: $2 \mu \mathrm{RT}$ buffer $10 \mathrm{x}$, $0.8 \mu \mathrm{l}$ dNTPs $25 \mathrm{x}, 1 \mu \mathrm{l}$ rev. transcriptase, and $4.2 \mu \mathrm{LEPC}-\mathrm{H} 2 \mathrm{O}$. The reaction was performed within a thermal cycler $\left(10 \mathrm{~min}: 25^{\circ} \mathrm{C}, 120 \mathrm{~min}: 37^{\circ} \mathrm{C}, 5 \mathrm{~min}: 85^{\circ} \mathrm{C}\right)$.

The cDNA was immediately used for qPCR or stored at $-20{ }^{\circ} \mathrm{C}$ until usage. Notably, high-concentrated samples (high RNA levels) were further diluted in DEPC-water up to $1: 10$, in order to guarantee enough starting material for subsequent qPCR reactions.

\section{3. qPCR}

qPCR was performed based on a TaqMan Assay platform. Every sample $(4 \mu \mathrm{l}$ of cDNA) was set up in triplicates with a mastermix (for 1 triplicate/gene), containing: 14 $\mu \mathrm{l}$ DEPC-water, $+2 \mu \mathrm{l}$ TaqMan probe (table 7), and $20 \mu \mathrm{l} 2 \mathrm{x}$ TaqMan master mix in a 96-well plate. Triplicates a $10 \mu \mathrm{l}$ were transferred into 384-well plates with a 8channel pipette, the plates were sealed and briefly vortexed, followed by quick centrifugation ( $<1 \mathrm{~min}, 1000 \mathrm{~g}$ ), before qPCR reactions were carried out within the ViiA7 system. Gene expression values were normalized to house-keeping genes $(U b c(+G a p d h))$ and calculated as relative expression levels based on the deltadeltaC $_{\mathrm{T}}$ method, wherein controls were set as 1. 
Table 7: TaqMan probes for qPCR

\begin{tabular}{|c|c|c|c|}
\hline gene & species & assay ID & resource \\
\hline Ccl17 & \multirow{9}{*}{$\begin{array}{c}\text { Mus } \\
\text { musculus }\end{array}$} & Mm01244826_g1 & \multirow{9}{*}{$\begin{array}{c}\text { Life } \\
\text { Technologies, } \\
\text { US }\end{array}$} \\
\hline $\mathrm{Ccl} 22$ & & Mm00436439_m1 & \\
\hline C3 & & Mm01232779_m1 & \\
\hline C3ar1 & & Mm02620006_s1 & \\
\hline Gapdh & & Mm99999915_g1 & \\
\hline Ifnb1 & & Mm00439552_s1 & \\
\hline$\| 12 b$ & & Mm01288989_m1 & \\
\hline Tnf & & Mm00443258_m1 & \\
\hline$U b c$ & & Mm02525934g1 & \\
\hline
\end{tabular}

G) RNA-Sequencing

\section{Sample preparation and sequencing}

Samples stored in TRIzol LS were transferred on dry ice, and further downstream processing was performed by Genewiz (New Jersey, US). In brief, RNA-Seq libraries were generated with the SMART-Seq preparation kit (CloneTech), and fragmented with the Nextera XT kit (Illumina). Libraries were pooled equimolar, and paired-end, 150 bp sequencing performed with an Illumina HiSeq2500 or 4000. Raw RNA-Seq data were delivered in FASTQ format.

\section{Downstream data processing}

Paired FASTQ data files per samples were used as input for the following steps conducted within the HUSAR platform provided by the DKFZ (Heidelberg, Germany). Data files were pre-processed with the EvalRSeq pipeline, and filtered for quality scores, poly-A trimming performance, $\mathrm{N}$ containing reads and artifacts were removed, and a clearing of rRNA contamination executed. Transcriptomes were mapped to the mouse genome (\# 38) using TopHat2 (v. 2.0.14). All of those steps are implemented in the EvalRSeq pipeline. Output BAM-files were used as input for the following pipeline, where the number of reads per gene were determined via genecode annotations (release VM14 (H2030), and VM23 (MDA, and 99LN)) with HTSeq-Count, based on HTSeq from Anders et al. (2015). HTSeq-Count files were used for downstream analysis with DESeq2. 


\section{RNA-Seq data analysis}

a) DESeq2: standard method for DEG generation

Every further analysis was performed within R, operated with RStudio. Most important step included the gene level analysis of RNA-Seq data, to compare expression levels between experimental conditions with DESeq2. High-throughput data files were annotated and merged to mouse gene names, ensemble IDs, and large data files were stored for further analyses, which also contained the statistical outputs (baseMean, log 2 fold change, IfcSE, stat, pvalue, and padj value) from a sample/condition to sample/condition comparison.

Importantly, adjusted $\mathrm{p}$ values (padj values) were used for statistics of data presented in here, which represent $p$ values corrected for multiple testing by using the Benjamini and Hochberg method by default as implemented within DESeq2.

MA and volcano plots were generated from raw data/normalized counts, which by default were set to be saved within this pipeline. Further downstream analyses were performed with VST data. These data were derived from variance-stabilized transformation (equals almost log2 transformation) of the normalized count data.

b) Further downstream analyses (pathway analysis, Venn and Euler plotting)

VST data were used for PCA clustering, heatmaps, and graphs representing relative expression values. Heatmaps were generated within the standard pipeline (see below) or with Heatmapper (Babicki et al., 2016), always using a Complete Linkage Clustering and Spearman Rank Correlation for distance measurement. Pathway analyses and gene annotations were performed on manually filtered top genes, always respecting a basemean greater than 20 (to filter for low/non-abundant genes), and a padj value less than 0.05 (= false discovery rate of $5 \%$ ).

Pathway analysis and gene annotation were performed with Metascape (Zhou et al., 2019), and/or clusterprofiler and enrichplot in RStudio. Venn diagrams were generated with Venny (Oliveros, 2007-2015), or/and Euler plots drawn via eulerR. TRRUST analysis was performed within Metascape, based on the original publication (Han et al., 2017). For gene list enrichments, all genes in the genome have been used as enrichment background. Terms with a $p$-value $<0.01$, a minimum count of 3 , and an enrichment factor $>1.5$ were collected and grouped into clusters based on 
their membership similarities (Metascape). All RNA-Seq data shown consist of at least 3 - 5 biological replicates per condition.

\section{Single Cell RNA-Seq}

For single cell RNA-Seq, samples were processed as described under E. The experiment was performed with TAM-MGs and TAM-MDMs out of 2 experimental conditions: treatment-naïve $\mathrm{H} 2030-\mathrm{BrM}$, and at d3 following 5 × 2 Gy WBRT (figure 5 ), since the TAM-MDM population at this time point already was found to constantly increase. TAMs were directly sorted into the plates with the Fusion, and plates were sealed again and stored on dry ice. Cell capture plates were further processed and data were analyzed by Single Cell Discoveries (SCD, Utrecht, Netherlands). Data were generated based on a SORT-Seq protocol as previously described (Muraro et al., 2016), and were analyzed with RacelD (Grün et al., 2016). Any downstream analyzes were performed on Poisson-, and UMI-corrected raw mapped reads, containing an UMI (unique molecular identifier) $>800$.

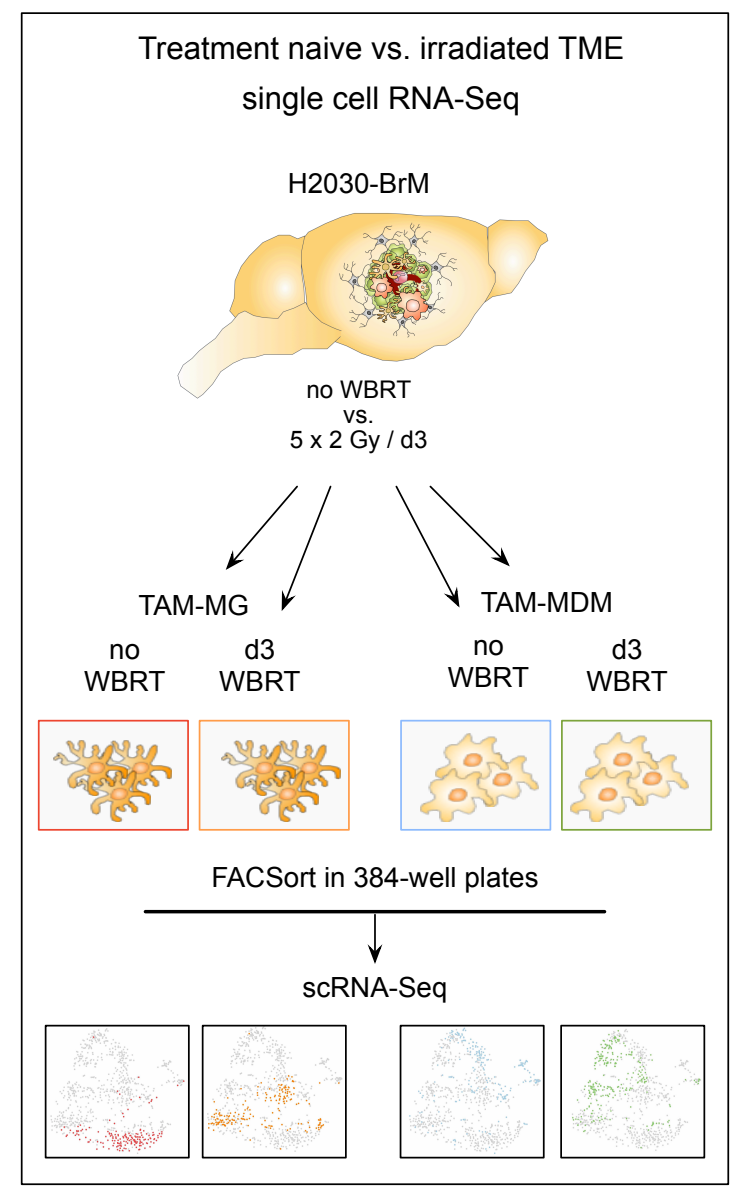

Figure 5: Single cell RNA-Seq of H2030-associated TAMs - experimental approach (modified from Schulz et al., 2020). 
H) Data, analysis and software

\section{Data sets generated/used in this thesis}

This part includes information solely related to RNA sequencing data, generated and utilized within this thesis.

All RNA-Seq data generated during this work and represented, including bulk and single cell data, have been stored in the common and publicly available repository GEO (Gene Expression Omnibus) of NCBI.

Data generated within this thesis include RNA-Seq data related to the H2030-BrM model, published in Schulz et al., 2020 available with the super-series accession number GSE137797.

Data derived from the 99LN-BrM model generated within this thesis are associated to Niesel et al., 2021, and can be found with GSE164049.

The data set containing RNA-Seq data of the MDA-BrM model is associated to Klemm et al. (in revision), and will be available with GSE133887.

RNA-Seq data of human BrM patients, which partially complement findings shown in this thesis have been published in Klemm et al. (2020), and can be accessed via https://joycelab.shinyapps.io/braintime/.

Note that some of the comparative multi-model graphs and data which consist of data points from all three sets will be part of the review: "TAMs in Brain Metastasis: Molecular signatures in mouse and man", of which the abstract has been accepted for the Research Topic: "The Role of the Immune Response in Brain Metastasis" in Frontiers in Oncology.

\section{Analysis, software and packages}

All data shown have been derived from and have been edited with various types of different software, briefly summarized within the following section.

Histological images have been derived from an Aperio ScanScope slide scanner and the network-based visualization tool Aperio eSlide Manager.

CQ1 image acquisition and processing was performed with the in-built CQ1 software. FCM and FACS data were acquired with BD FACS Diva software, and data have been transferred to and analyzed within FlowJo. qPCRs were performed with the ViiA7 system, and data were exported and further analyzed within Excel. Data are represented as relative quantity values $(R Q)$, based on normalization to the control 
(= 1). Both, flow and $\mathrm{QPCR}$ data were furthermore plotted and analyzed within GraphPad Prism. All RNA-Seq data have been analyzed as described above (see G.3, script below), and significance is derived from padj values. If not indicated otherwise, data shown here contain either normalized counts ("raw data") or differentially expressed genes (DEGs) extracted from cell-cell comparisons with a basemean $(B M)>20$ and adjusted $p$ value (padj.) $<0.05$. Correlation of flow data with BrM stage was performed in Prism, using Pearson correlation coefficient and two-sided test. Significance was always assumed with $p$ at least $<0.05$.

All software and packages used in here are described in their corresponding methods sections, and are summarized in the resource identifier table (table 1).

\section{Example script for RNA-Seq data analysis}

The following script is a summarized compilation of all single pipelines used for the generation of indicated data and data plots. Every package used in here can be found in the resource identifier table (table 1). 
DESEQ2, PCA plots, Heatmaps, VST

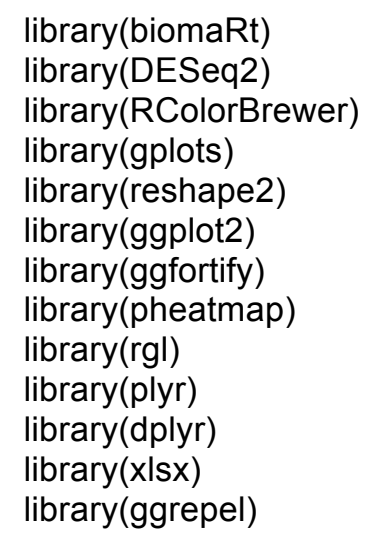

\#\#\# read annotation file \#\#\#

Annot<- read.csv2("annotation file.csv") nrow(Annot)

Annot\$ensemblgene_ID[Annot\$ensemblgene_ID=="'] $]<-N A$

Annot <- Annot[!is.na(Annot\$ensemblgene_ID), ]

Annot $<-$ Annot\% $>\%$ distinct(ensemblgene_ID, .keep_all = TRUE)

\#\#\#alternative annotation\#\#\#\#

ForAnnotation<- read.csv2("annotation file.csv", header = FALSE, sep=";") head(ForAnnotation)

row.names(ForAnnotation)<- ForAnnotation $\$ V 1$

head(ForAnnotation)

is.data.frame(ForAnnotation)

nrow(ForAnnotation)

names(ForAnnotation)<-c("ensembl_gene_id", "ensembIID")

head(ForAnnotation)

ensembl = useMart(biomart="ensembl", dataset="mmusculus_gene_ensembl")

mart_mouse<-useDataset("mmusculus_gene_ensembl", useMart("ensembl"))

MouseAnnotations<- getBM(filters= "ensembl_gene_id",

attributes=c("ensembl_gene_id",

"external_gene_name"),

values=row.names(ForAnnotation), mart= mart_mouse)

HumanOrthologAnnotations<- getBM(filters= "ensembl_gene_id", attributes=

c("ensembl_gene_id","hsapiens_homolog_ensembl_gene"),\#"ensembl_gene_id",

head(HumanOrthologAnnotations) values=row.names(ForAnnotation), mart= mart_mouse)

nrow(HumanOrthologAnnotations)

HumanOrthologAnnotations\$hsapiens_homolog_ensembl_gene[HumanOrthologAnnotations\$hsapien s_homolog_ensembl_gene=="'"]<- NA

HumanOrthologAnnotations <-

HumanOrthologAnnotations[!is.na(HumanOrthologAnnotations\$hsapiens_homolog_ensembl_gene),]

HumanOrthologAnnotations<-

HumanOrthologAnnotations\%>\%distinct(hsapiens_homolog_ensembl_gene,

keep_all = TRUE)

row.names(HumanOrthologAnnotations)<-

HumanOrthologAnnotations\$hsapiens_homolog_ensembl_gene

head(HumanOrthologAnnotations)

mart_human<- useDataset("hsapiens_gene_ensembl", useMart("ensembl"))

ensembl = useMart(biomart="ensembl", dataset="hsapiens_gene_ensembl")

genenames<-row.names(HumanOrthologAnnotations) 
HumanAnnotations<- getBM(filters= "ensembl_gene_id", attributes= c("ensembl_gene_id", "hgnc_symbol"), values=genenames, mart= mart_human)

MouseAnnotations[1:10,]

HumanAnnotations[1:10,]

names(HumanAnnotations)<-c("hsapiens_homolog_ensembl_gene", "human_hgnc_symbol") head(HumanAnnotations)

nrow(HumanOrthologAnnotations)

nrow(HumanAnnotations)

HumanAnnotations\$hsapiens_homolog_ensembl_gene[HumanAnnotations\$hsapiens_homolog_ense mbl_gene=="']<- NA

HumanAnnotations <-

HumanAnnotations[!is.na(HumanAnnotations\$hsapiens_homolog_ensembl_gene),]

HumanAnnotations<- HumanAnnotations \% $\%$ distinct(hsapiens_homolog_ensembl_gene, .keep_all = TRUE)

HumanAnnot<- join(HumanOrthologAnnotations, HumanAnnotations, type= "left")

nrow(HumanAnnot)

head(HumanAnnot)

HumanAnnot\$ensembl_gene_id[HumanAnnot\$ensembl_gene_id=="']<- NA

HumanAnnot <- HumanAnnot[!is.na(HumanAnnot\$ensembl_gene_id),]

HumanAnnot<- HumanAnnot\%>\%distinct(ensembl_gene_id, .keep_all = TRUE)

nrow(HumanAnnot)

nrow(MouseAnnotations)

Annot_H_M<- join(MouseAnnotations, HumanAnnot, type = "left")

$\operatorname{nrow}($ Annot_H_M)

nrow(ForAnnotation)

Annot<- join(ForAnnotation, Annot_H_M, type = "full")

$\operatorname{nrow}($ Annot)

head(Annot)

\#\#\#removing duplicates\#\#\#

nrow(MouseAnnotations)

MouseAnnotations\$ensembl_gene_id[MouseAnnotations\$ensembl_gene_id=="']<- NA

MouseAnnotations <- MouseAnnotations[!is.na(MouseAnnotations\$ensembl_gene_id),]

MouseAnnotations<- MouseAnnotations \% $\% \%$ distinct(ensembl_gene_id, .keep_all = TRUE)

\#\#\#Read TXT file with data to compare (one of them should be called Control)\#\#\#

samples <- read.table('HT_Seq_Count_Files/samples_XYZ/Experiment1_SamplesAvsB.txt', header=TRUE, sep='It')

samples\$group <- relevel(samples\$group, ref = "Control")

head(samples)

\#\#\# DESeq Run \#\#\#

ddsHTSeq <- DESeqDataSetFromHTSeqCount(sampleTable=samples, design= $\sim$ group)

dds $<-$ DESeq(ddsHTSeq, betaPrior $=$ TRUE)

head(counts(dds))

res<-results(dds)

\section{\#\#\#NORMALIZED COUNTS\#\#\#\#}

foo $<$ - counts(dds, normalized $=$ TRUE)

write.csv(foo, file $=($ paste 0 (format(Sys.Date) ),

"\%Y\%m\%d"),"_NORMcounts_Experiment1_SamplesAvsB.csv")))

\#\#\#Combine Data in Data Frame\#\#\#

dataframe_res<-as.data.frame(res)

dataframe_res <- cbind("ensemblgene_ID" = rownames(dataframe_res), dataframe_res)

rownames(dataframe_res) <- NULL 
\#\#\# Annotation of Readcounts \#\#\#

$\mathrm{df}<-$ counts(ddsHTSeq)

$\mathrm{df}<-$ as.data.frame $(\mathrm{df})$

df <- cbind("ensemblgene_ID" = rownames(df), df)

rownames(df) <- NULL

names(Annot)<- c("ensembl_gene_id", "ensemblgene_ID", "Gene_name")

Annotations_HTSeq_counts<- merge(Annot, df, by= "ensemblgene_ID")

head(Annotations_HTSeq_counts)

AllResults<- join(Annotations_HTSeq_counts,dataframe_res, type="full")

\#\#\#Safe Data Frame\#\#\#

write.csv2(AllResults, row.names = FALSE, file=( paste0(format(Sys.Date(), "\%Y\%m\%d"),"_

Experiment1_SamplesAvsB.csv")))

\#\#\#Plotting\#\#\#

AllResults<- read.csv2("Date_Experiment1_SamplesAvsB.csv", header = TRUE)

\#\#\#MA Plots\#\#\#

pdf( paste0(format(Sys.Date(), "\%Y\%m\%d"),"_MA_Experiment1_SamplesAvsB.pdf"))

$\operatorname{par}(\mathrm{mai}=\mathrm{c}(1,1,1,0.5))$

plotMA(res, alpha= 0.05, colNonSig="Color", colSig= " Color ", colLine=NULL, cex.lab = 2,

cex.axis=2, ylab= "log2 Fold Change", ylim=c(-15,15), xaxt="n", yaxt="n",

main= "NAME in PLOT")

abline $(\mathrm{h}=0$, col= " Color ")

axis $(2$, at= c(--15,-14,-13,-12,-11,-10,-9,-8,-7,-6,-5,-4,-3,-2,-1,0,1,2,3,4,5,6,7,8,9,10,11,12,13,14,15),

las $=1$, cex.axis=2), ticks <- seq $(-2,5$, by=1), labels <- sapply(ticks, function(i)

as.expression(bquote $\left(10^{\wedge}\right.$.(i))))

axis $(1$, at $=c(0.01,0.1,1,10,100,1000,10000,100000)$, labels=labels, cex.axis=2)

legend('topright', Iwd=c(2.5,2.5), c ("padj > 0.05", "padj < 0.05"), col = c("Color ", " Color "),

dev.off()

bty $=" n "$, Ity $=0, p c h=16$, cex=2)

\section{\#\#\# Volcano Plots\#\#\#}

pdf( paste0(format(Sys.Date(), "\%Y\%m\%d"),"_Volcano_Experiment1_SamplesAvsB.pdf"))

$\operatorname{par}(\mathrm{mai}=\mathrm{c}(1,1,1,0.5))$

plot(AlIResults\$log2FoldChange, -log10(AlIResults $\$ p$ value), $p c h=20, x \lim =c(-10,10)$,

ylim $=c(0,50), x l a b=$ "log2 Fold Change", ylab= "-log10 $(p$ value)", las $=1$, cex.lab=1, cex.axis=1, main = "NAME in PLOT")

with(subset(AllResults, padj<0.05 ), points(log2FoldChange, -log10(pvalue), pch=20, col="gray60"))

legend('topleft', Iwd=c(2.5,2.5),

c ("p adj > 0.05", "p adj < 0.05"), col = c("black", "gray60"), bty="n", Ity= 0, pch = 16) dev.off()

\#\#\#ST generation and storage of VST data file\#\#\#

vst $=$ varianceStabilizing Transformation(dds, blind=TRUE )

vst_transf_Werte <- assay(vst)

vst_transf_Werte <- as.data.frame(vst_transf_Werte)

head(vst_transf_Werte)

names(vst_transf_Werte) <- c("sample1_vst", "sample2_vst", "sampleN_vst")

vst_transf_Werte\$ensemblgene_ID <- row.names(vst_transf_Werte)

vst_transf_Werte_AllRes <- join(AllResults, vst_transf_Werte, type = "left")

head(vst_transf_Werte_AllRes)

write.csv2(vst_transf_Werte_AllRes, row.names $=$ TRUE, file= $($ paste $0($ format $($ Sys.Date () ,

"\%Y\%m\%d"),"_VST_Experiment1_SamplesAvsB.csv"))) 


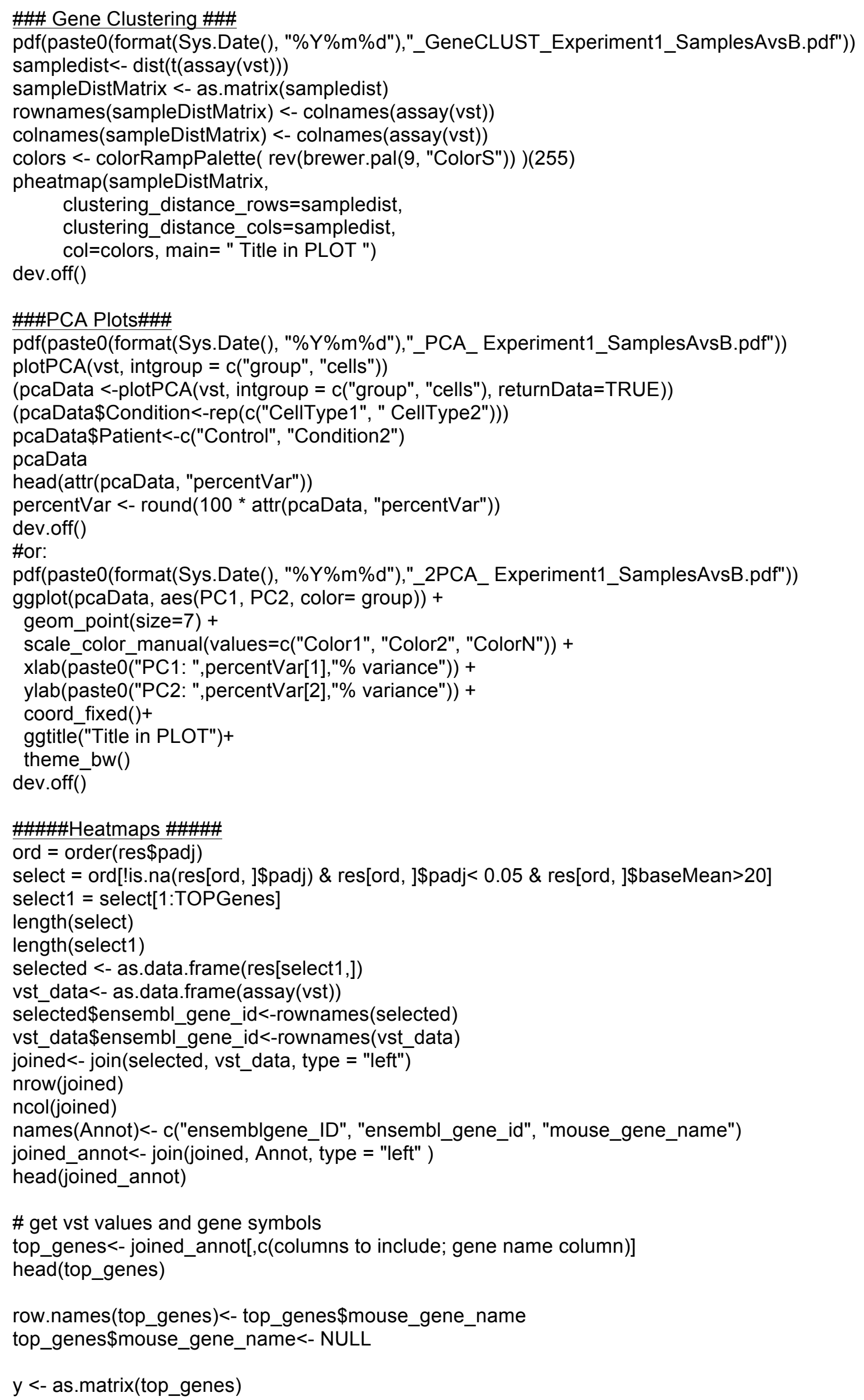


hr <- hclust(as.dist(1-cor(t(y), method="spearman")), method="complete")

hc $<-$ hclust(as.dist(1-cor(y, method="spearman")), method="complete")

$\mathrm{hr}<-$

my_palette <- colorRampPalette("ColorsXYZ"))(n = 299)

pdf(paste0(format(Sys.Date(), "\%Y\%m\%d"),"_Heatmap_TopGenesPADJ=0.05

Experiment1_SamplesAvsB.pdf"))

heatmap.2(y,

Rowv $=$ as.dendrogram $(\mathrm{hr})$,

Colv $=$ as.dendrogram $(\mathrm{hc})$,

col= my_palette,

scale="row", trace="none", margin=c $(6.8,8)$,

$\mathrm{cexCol}=0.8$,

srtCol $=45$,labRow $=$ row.names $(y)$,

dev.off()

main = "Title in PLOT")

\#my_palette <- colorRampPalette(c("\#2668A8", "\#ffffbf", "\#CB0017"))(n = 299)
\#my_palette <- rev(brewer.pal(11, "PiYG"))
\#my_palette <- color = colorRampPalette(c("navy", "white", "firebrick3"))(50)

\#or:

pdf(paste0(format(Sys.Date(), "\%Y\%m\%d"),"_2Heatmap_Experiment1_SamplesAvsB.pdf"))

pheatmap $(y$,

cluster_rows $=\mathrm{T}$, cluster_cols $=\mathrm{T}$,

Rowv $=$ as.dendrogram $(\overline{h r})$,

Colv $=$ as.dendrogram(hc),

\#col= colorRampPalette(c("ColorsXYZ"))(50),

\#col = my_palette

scale $="$ row", trace $="$ none", $\operatorname{margin}=c(5,20)$,

fontsize_row $=6$,

cexCol $=0.3$,

border_color $=$ "Color",

srtCol=45,labRow= row $\cdot$ names $(y)$,

\#cutree_rows $=2$,

\#cutree_cols $=2$,

dev.off()

main $=$ " Title in PLOT ")

\section{EULERPLOTS}

library(eulerr)

\section{\#\#\#2 parts\#\#\#\#}

fit <- euler(c("A" = 1234, "B" = 321, "A\&B" = 123))

or

\#\#\#3 parts\#\#\#\#

fit <- euler(c("A" = 123, "B" = 234, "C" = 345, "A\&B" = 12, "A\&C" = 23, "B\&C" = 34, "A\&B\&C" = 45))

\# Customize colors, remove borders, bump alpha, color labels white

pdf( paste0(format(Sys.Date(), "\%Y\%m\%d"),"_EULER_nameXYZ.pdf"))

plot(fit, quantities = TRUE, fills = list(fill = c("color ", " color ", $(+$ color 3$))$, alpha $=0.3)$,

labels $=$ list $(\mathrm{col}=$ " color ", font $=8$, fontsize $=5)$ )

dev.off() 


\section{CLUSTERING and ENRICHMENT}

library(clusterProfiler)

\#\#\#Change organism of interest --> Mm or Hs \#\#\#\#

organism = "org.Mm.eg.db"

library (organism, character.only $=$ TRUE)

library(enrichplot)

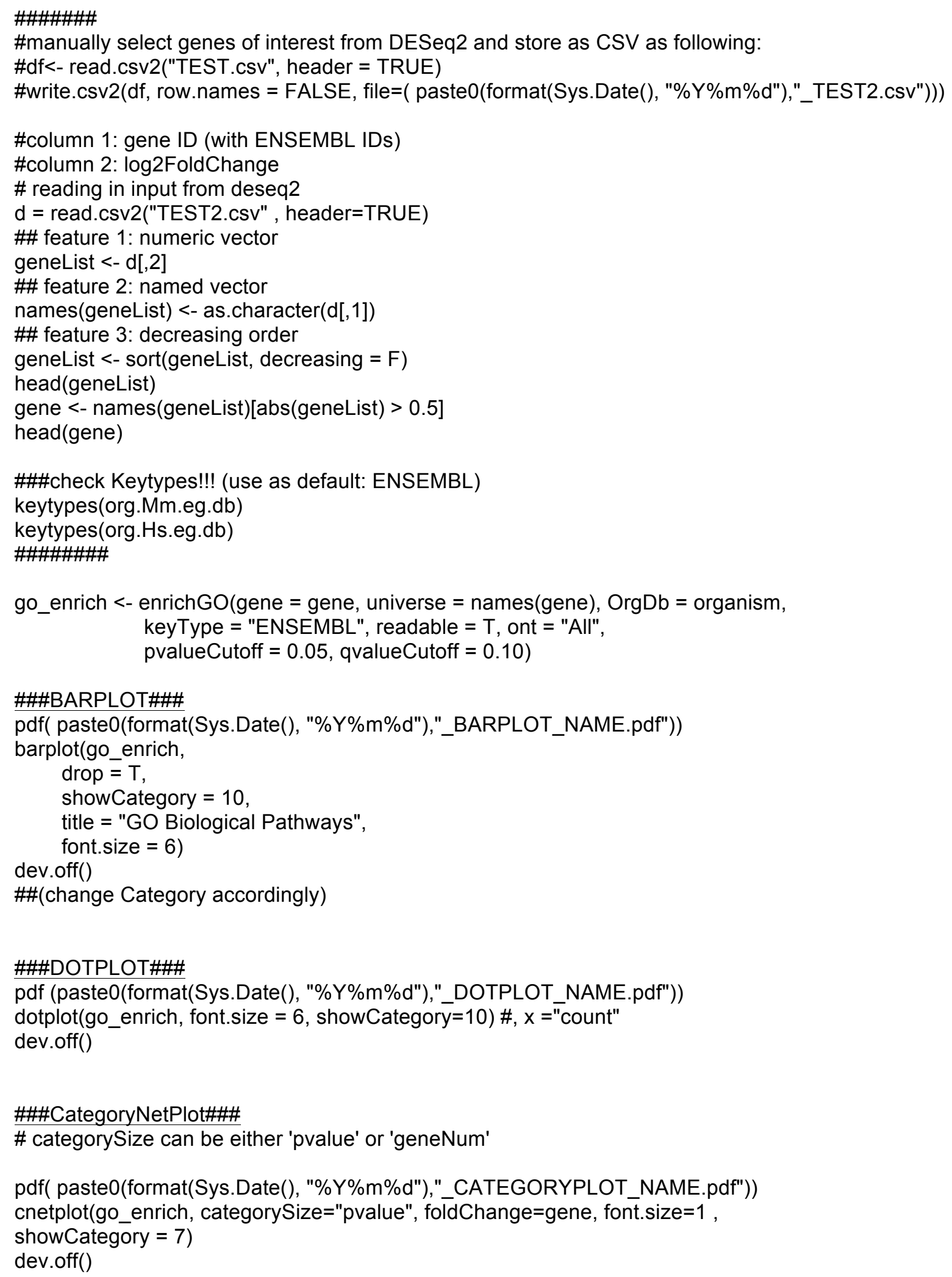




\section{Results}

A) Brain metastasis induce changes within the spatial organization of brain-resident cell types and induce activation of glial cells

While the brain represents a unique organ with highly specialized cell types, BrM induce profound changes within its cellular organization. In order to investigate these effects, different cell types of the BrM TME at different stages of tumor progression were assessed by immuno-histochemistry in a syngeneic mouse model (99LN-BrM) (figure 6), particularly focusing on brain-resident cell types. While neurons showed basically no activation or infiltrating pattern towards small BrM of only a few hundred cells (figure 6a), astrocytes showed an apparent upregulation of the astrocyte activation marker GFAP (figure 6a), and delineation of BrMs. Staining for TAMs with the pan-macrophage marker IBA1 showed that those cells surrounded small BrM as well, but also infiltrated tumor cell cluster. While tumor progression continued to result in neuronal cell body displacement and astrocytic delineation, IBA1 staining in larger 99LN-BrM revealed an accumulation of IBA $1^{+}$cells (TAMs) (figure 6a) (Chae et al., 2019). Similarly, cellular changes of non-infiltrating neurons and astrocytes can be found via immuno-fluorescence stainings in a melanoma BrM model (H1_DL2), and lung cancer BrM model (H2030) (figure 6b). Together the histological examinations revealed that brain-resident cell types show two pattern in BrM: nonimmune, resident cells (here $\mathrm{NEUN}^{+}$neurons and $\mathrm{GFAP}^{+}$astrocytes) were not infiltrative, whereas IBA $1^{+}$TAMs showed clear activation pattern as seen in a typical change of morphology (Olah et al., 2011) from a ramified into an amoeboid phenotype (figure 7a).

While the TAM population seems to increase during tumor progression, double immuno-fluorescence staining of IBA1 and TMEM119 across sections from all three models (H1_DL2, H2030, 99LN) further revealed the existence of two major subpopulations within the TAM population (figure $7 \mathrm{~b}$ ). While IBA $1^{+} / \mathrm{TMEM} 19^{+}$cells surround BrM, IBA $1^{+} / \mathrm{TMEM} 119^{-/ l o w}$ cells infiltrate BrMs more apparent (figure $7 \mathrm{~b}$ ), indicating the recruitment of peripheral macrophages. 


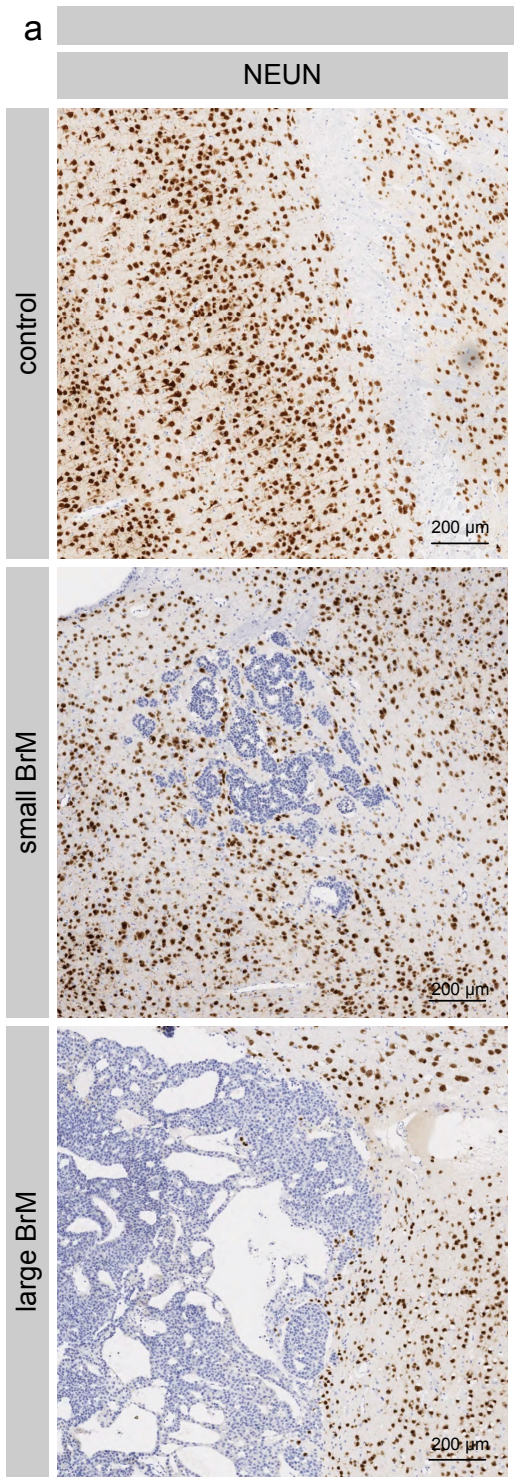

99LN (breast-to-brain metastasis)
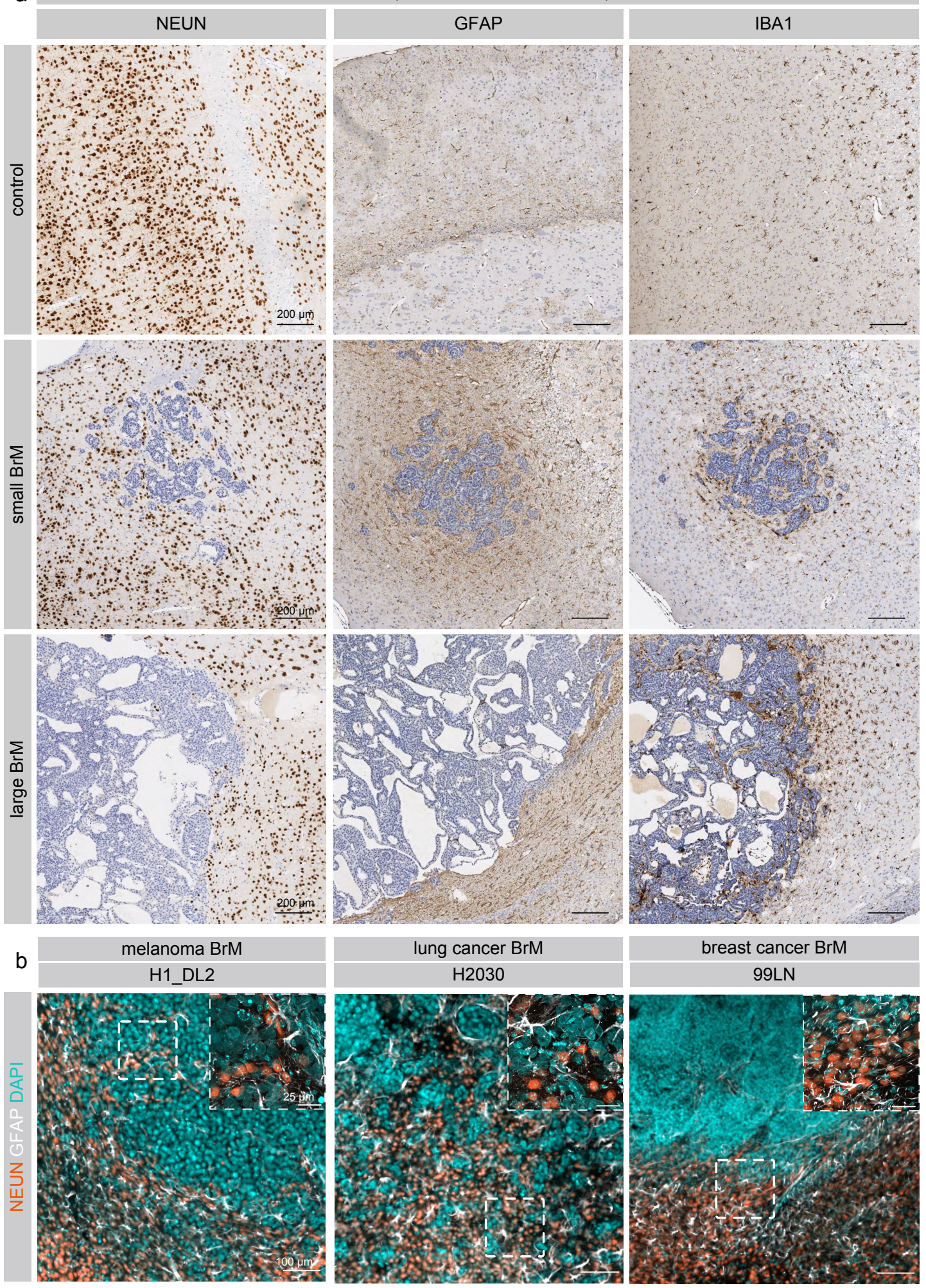

Figure 6: Distribution of brain-resident cell types in BrM. a) IHC stainings for neurons (NEUN), astrocytes (GFAP), and microglia/macrophages (IBA1) in control slides, and slides with small (middle), or large (bottom) BrM. Scale bar $=200 \mu \mathrm{m}$. b) Representative immunofluorescence double-stainings for NEUN and GFAP in the indicated BrM models. Scale bar $=100 \mu \mathrm{m}$, inlay $=25 \mu \mathrm{m}$. 
a

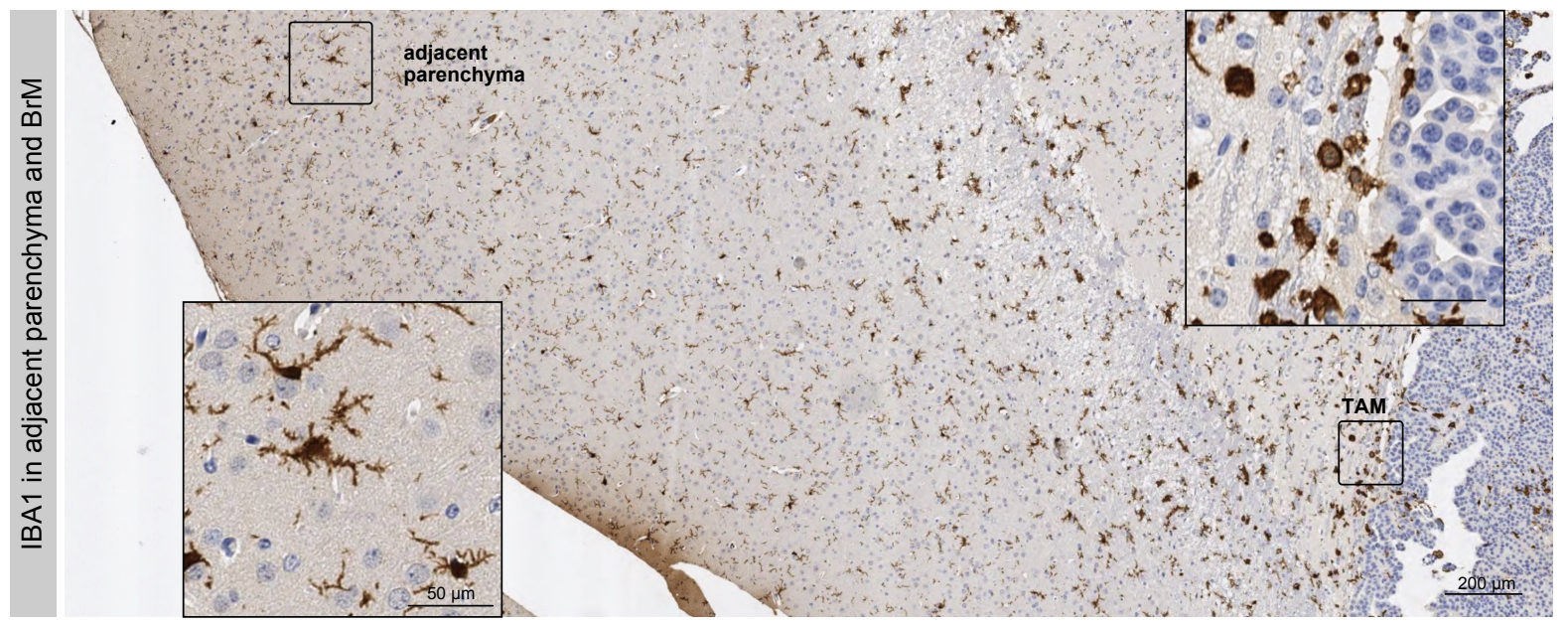

b
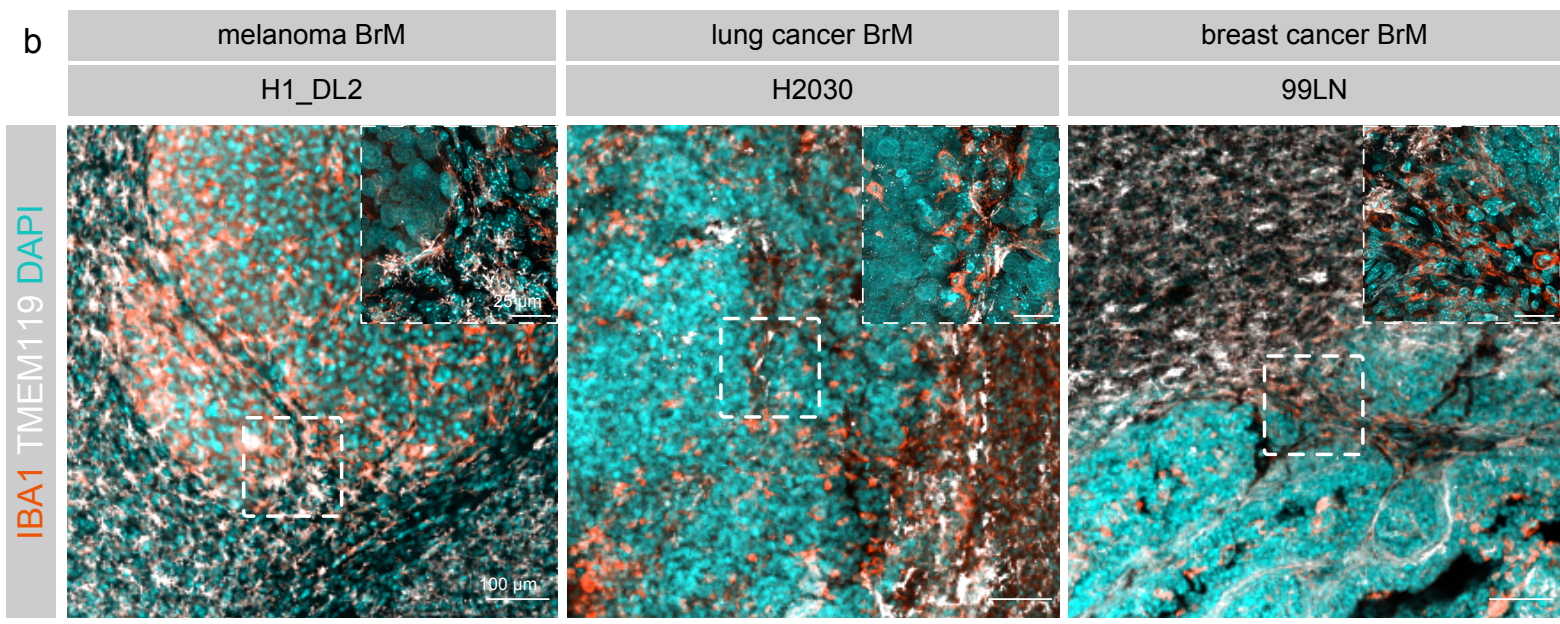

Figure 7: Immuno-stainings of microglia/macrophages in BrM. a) IHC staining for IBA1 (microglia/macrophages) on a 99LN BrM-containing slide; with a close-up of different activation states, ramified microglia in adjacent brain parenchyma (left), and amoeboid/activated in close association with BrM (right). Scale bar $=200 \mu \mathrm{m}$, inlay $=$ $50 \mu \mathrm{m}$. b) Representative immunofluorescence double-stainings for IBA1 (red) and TMEM119 (white) in the indicated BrM models. Scale bar $=100 \mu \mathrm{m}$, inlay $=25 \mu \mathrm{m}$. 
B) Immune cell infiltration into BrM

\section{TAMs represent a major stromal compartment of BrM}

In order to further understand the dynamic changes within the myeloid immune cell compartment with its different TAM populations during BrM progression, flow cytometry (FCM) was performed. The panel included markers to examine the four major myeloid immune cell types TAM-MG, TAM-MDM, inflammatory (infl.) monocytes, and granulocytes in the BrM-TME derived from the entities most frequently metastasizing to the brain: melanoma (H1_DL2), lung cancer (H2030), and breast cancer (99LN). BrM progression was monitored by imaging, and mice were stratified according to their BLI signal (H1_DL2, H2030), or MRI image-based BrM volume (99LN) (figure 8a). Noteworthy, the H2030 model was not appropriate to image via MRI due to the huge amount of bleeding and hemorrhage within those BrMs (see figure 17a,b).

The total myeloid cell population was defined as positive for CD45/CD11b, from which three major sub-groups were separated based on their Ly6C/Ly6G abundance:

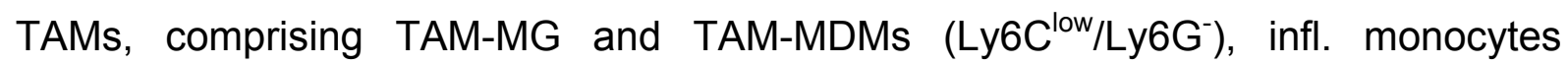

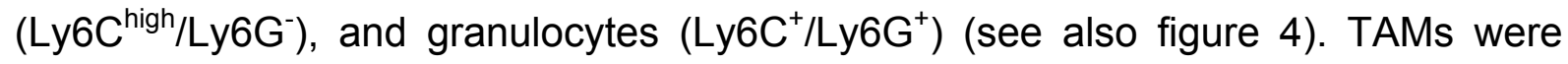
further divided into TAM-MGs and TAM-MDMs based on the integration of CD49d (Bowman et al., 2016). Encoding an integrin, this marker has been identified and validated as specific for peripheral-derived macrophages in the myeloid cell compartment of brain tumors, based on the evaluation of lineage tracing modelbased RNA-Seq data of preclinical brain tumor models (Bowman et al., 2016).

The melanoma BrM model H1_DL2 showed very low to almost no infiltration of granulocytes (mean across all replicates $0.2 \%$ of CD45/CD11b cells), or infl. monocytes (mean $=0.31 \%$ of CD45/CD11b cells) within lesions of different sizes (figure $8 b$ ). Similarly, TAM-MDMs were less abundant during BrM progression from small to large BrM, with an approximate mean of $2 \%$ to $5.3 \%$ of CD45/CD11b/Ly6C low/Ly6G $^{-}$cells, respectively. Technically however H1_DL2 BrMs were macro-dissected, and since every mouse harbored several dozens of micrometastases (as represented in figure 8a), the examined BrM tissues did not fully correlate with the BLI values derived from whole brain. Thus, data shown here might miss a massive influx in very late BrM stages, which however could not be achieved since mice also developed extra-cranial metastases. In contrast, FCM data of the 
other two BrM models displayed a strong tendency: BrM progression induced massive recruitment of granulocytes in H2030 (Schulz et al., 2020), and 99LN (figure $8 \mathrm{~b}, 8 \mathrm{c})$. Although the relative quantities of cell populations were very similar and not significantly different in $\mathrm{H} 2030$ (Schulz et al., 2020), the granulocyte population increased from approximately $8 \%$ (mean) of $C D 45^{+} / C D 11 b^{+}$cells in small lesions to approximately $18 \%$. Infiltration of granulocytes in breast cancer BrM (99LN) was less pronounced but increased from $1.4 \%$ (mean in small stages) to $6.1 \%$ (mean in large stages) during tumor progression, as well. The number of infl. monocytes remained almost stable during tumor progression in $\mathrm{H} 2030$ (from $6.3 \%$ to $8.7 \%$ ), and 99LN (from $3 \%$ to $2.7 \%$ ) (figure $8 \mathrm{~b}$ ). Most apparent within the myeloid compartment was the tendency towards continuous influx of TAM-MDMs across all models, concurrently with a relative decrease of the TAM-MG population (figure $8 \mathrm{~b}$ ). While H1_DL2 recruited only few amounts of TAM-MDMs (figure 8b), H2030 and 99LN showed a strong infiltration of TAM-MDMs during BrM progression. The percentage of MDMs within the whole TAM population was positively correlated $(p<0.05)$ with both, increasing BLI values in $\mathrm{H} 2030$ ( $r=0.9015$, p: 0.0141), and with final BrM volumes in 99LN ( $r=0.9104, p: 0.0117$ ) (figure 8c). In addition to an increasing TAM population during BrM progression, different spatial localization of IBA1 $1^{+} / T M E M 119^{-}$, and IBA $1^{+} / \mathrm{TMEM} 19^{+}$cells was appreciated via IF in 99LN-BrMs (figure 8d). Interestingly, large 99LN-BrM showed an apparent trend towards more infiltrating IBA $1^{+} / \mathrm{TMEM} 119^{+}$cells.

Together the data show that while the majority of myeloid cells in non-BrM control mice consist of MGs, the myeloid compartment in $\mathrm{BrM}$ is constantly changing during tumor progression across different models. This was most apparent within a constant increase of the TAM-MDM population (figure 8b,c). 


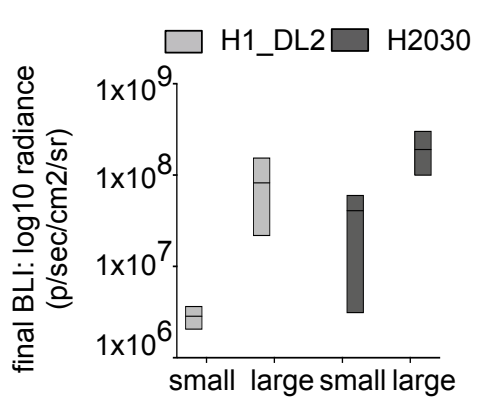

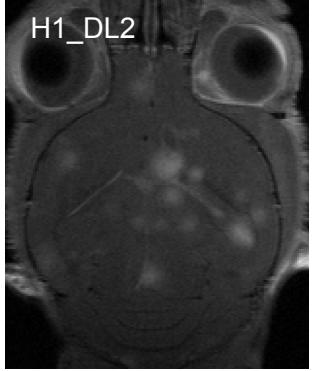

H2030
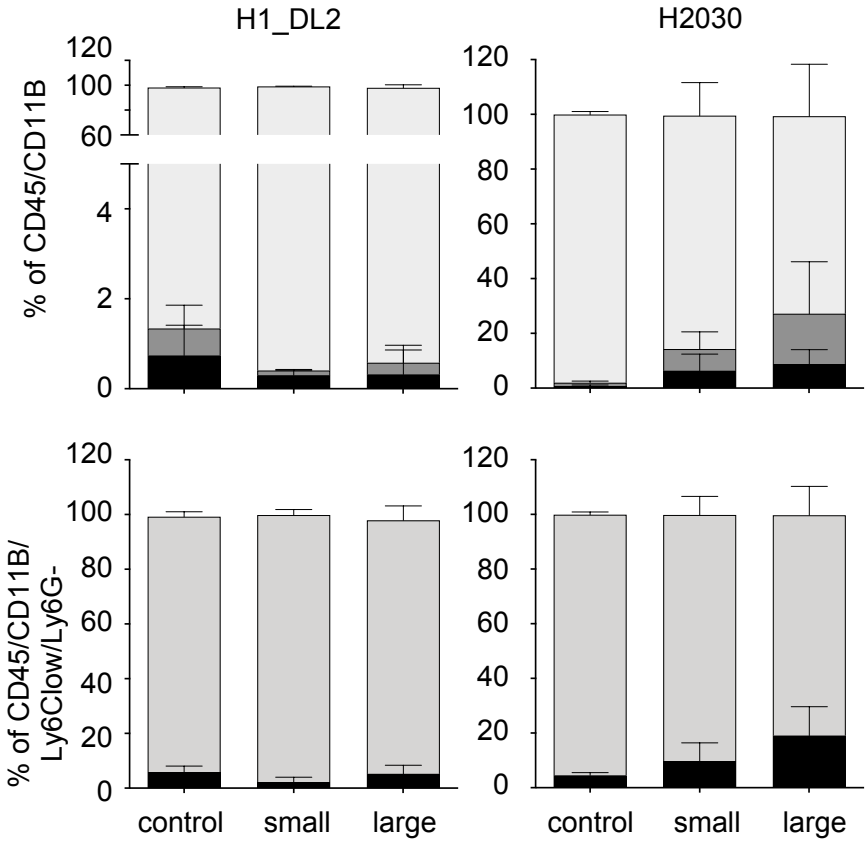

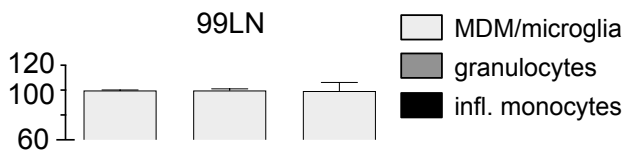

C

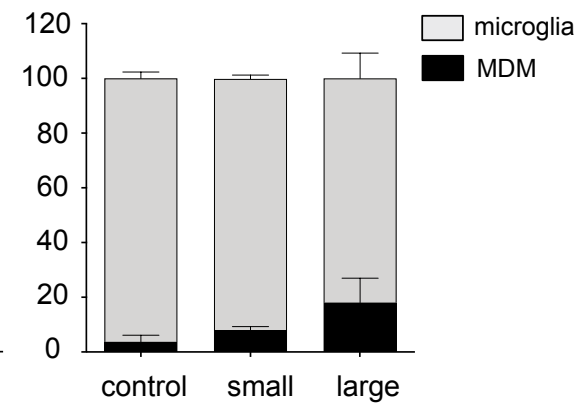

H2030
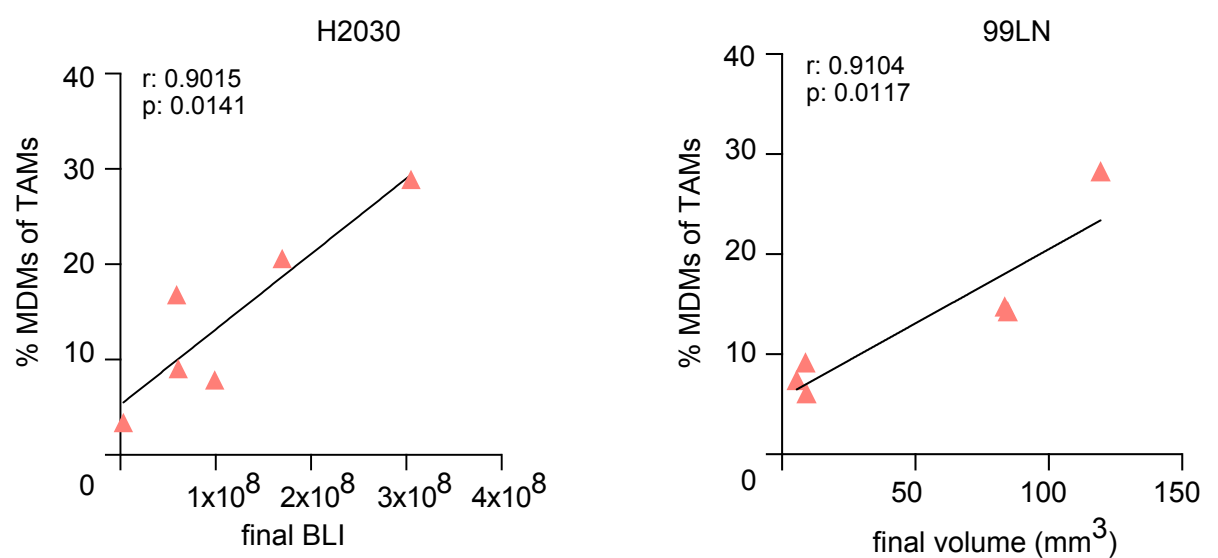

d
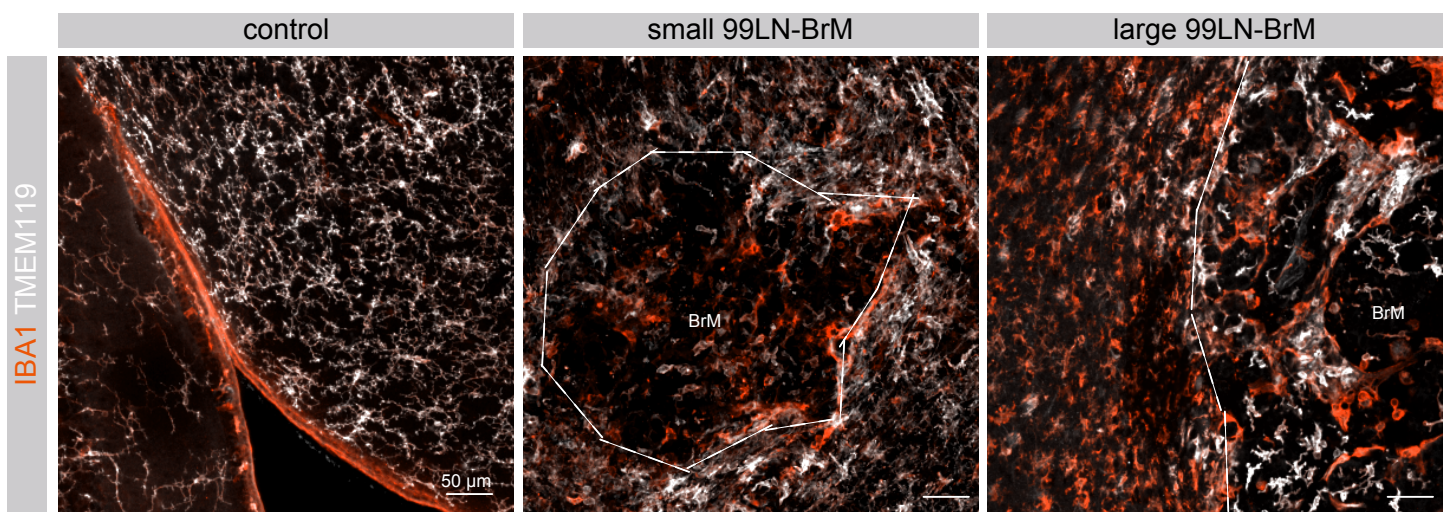
Figure 8: The dynamic myeloid immune cell compartment within the TME of different BrM models. a) Final BrM values of mice for FCM; H1_DL2 and H2030 xenograft BrM models were stratified based on BLI values (as radiance), whereas 99LNbearing mice were stratified based on final MR image volumina. Additionally, representative MR images of H1_DL2 and 99LN are shown. b) Relative quantification of myeloid cell populations in normal brain (control), and in three different BrM models of small and large stages by flow cytometry ( $n=3$ for every condition in every model, apart from $\mathrm{n}=2$ in small H1_DL2). All H1_DL2 and H2030 BrM samples were macro-dissected, or full brains of $99 \mathrm{LN}-\mathrm{BrM}$ used for FCM. c) Percentage of MDMs among TAMs correlated to final $\mathrm{BLI}$ values (for $\mathrm{H} 2030$ ), or final MRI-based BrM volumina (99LN). d) Representative immunofluorescence doublestaining for IBA1 (red) and TMEM119 (white) in control brain slice, and small or large 99LN-BrM, respectively. Scale bar $=50 \mu \mathrm{m}$. Note that data derived from H2030 have been partially or completely published in Schulz et al., 2020.

\section{Brain slice assay and live cell imaging mimics a dynamic BrM TME}

In order to gain insight into the in vivo-like dynamics of TAMs within the BrM-setting, a brain slice-based protocol was modified and a multi-cellular culture system developed during this thesis (manuscript in preparation). To allow constant tracking of tumor cells during longer live cell imaging experiments, a stable blue fluorescent protein (BFP)-expressing tumor cell line was generated (99LN-BFP). These cells together with in vitro differentiated, Cell Tracker-labelled macrophages (BMDMs) were used on brain slices of different transgenic mice backgrounds in order to examine the cellular behavior of those cell types.

Live cell imaging of a particular slice region of CX3CR1-GFP mice, which stain for MGs in the normal brain, revealed dynamic interactions between all cell types of interest as seen by a high cell motility. Both, tumor cell-BMDM (figure 9a, star) as well as MG-BMDM crosstalk (figure 9a, arrow) was observed during the imaging period of $24 \mathrm{~h}$ (figure 9a). Especially BMDMs appeared very motile in the presence of 99LN tumor cells (figure 9b). In contrast to TAMs, astrocytes (here GFAPtdTomato) showed very few obvious cellular movements within another live cell experiment. Surprisingly however, one field of view revealed the presence of two GFAP $^{+}$cells, which seemed to be attracted by 99LN-BFP tumor cells (figure 9c), as seen by a directed movement of red cell bodies towards the blue tumor cells within an imaging period of $24 \mathrm{~h}$. 
Together this data underline the dynamics of the TME, which in part can be attributed to high cell motility of different cell types. Hence, this approach might allow to more precisely determine molecular changes associated to distinct behavior or infiltration pattern, or vice versa, in the future.
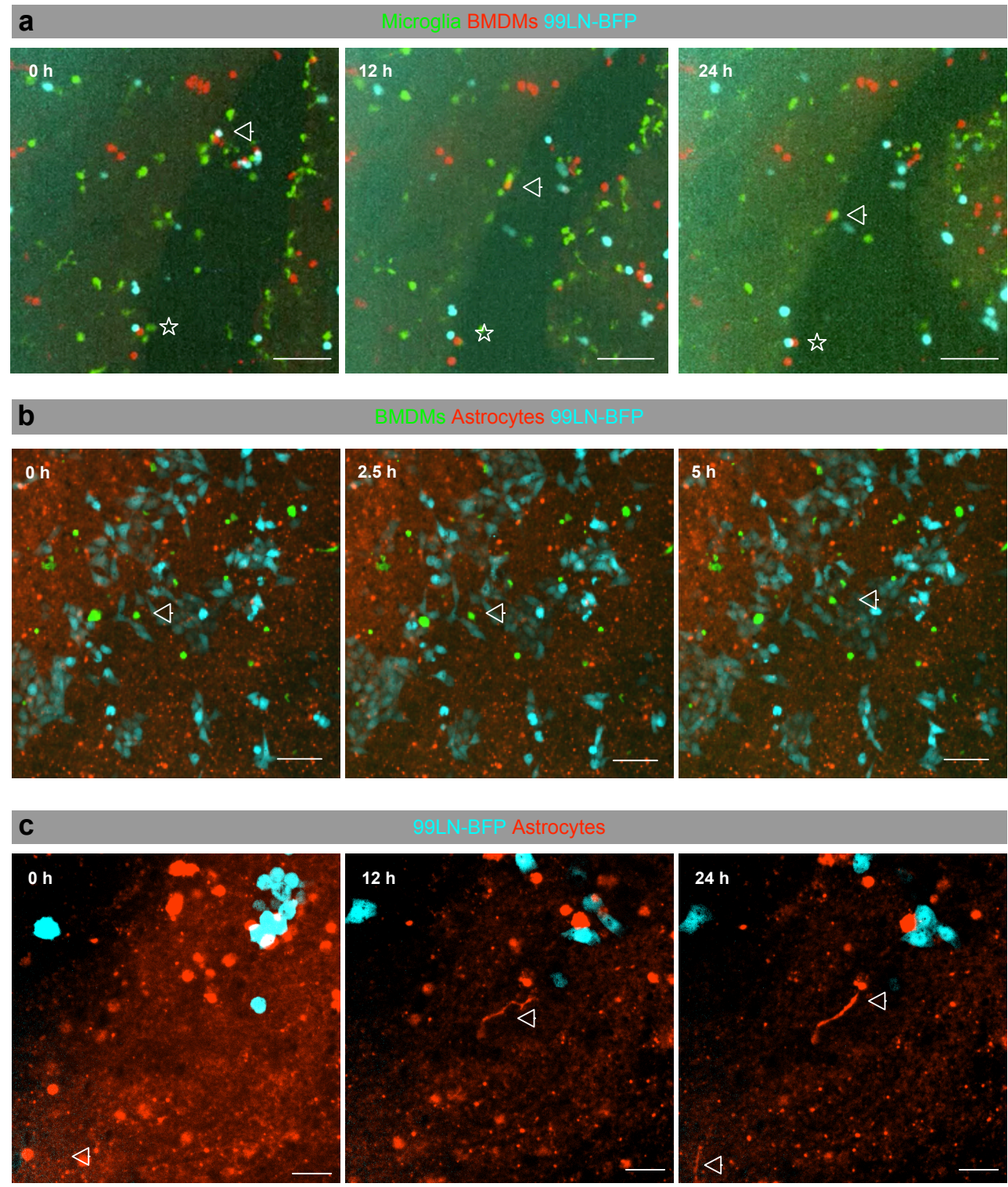

Figure 9: Triple culture brain slice live imaging reveals a highly dynamic microenvironment in the presence of tumor cells. a) Initial triple-culture experiment where 99LN-BFP tumor cells (blue) have been applied, and shortly thereafter cell tracker red-labelled BMDMs on top of brain slices from healthy CX3CR1-GFP/WT mice $($ microglia $=$ green). Live-imaging was started about $3 \mathrm{~h}$ following application of the cells, and was performed every $15 \mathrm{~min}$ for in total $24 \mathrm{~h}$. Scale bar $=100 \mu \mathrm{m}$. b) Similar set-up as in a, but on another background, with red-labelled astrocytes (GFAP-Cre x R26_tdTomato), green labelled BMDMs, and imaging was performed for only $5 \mathrm{~h}$. Scale $\overline{b a r}=100 \mu \mathrm{m}$. c) Live cell imaging of 99LN-BFP tumor cells (blue) on a brain slice of an astrocyte reporter line (GFAP-Cre x R26_tdTomato). Live imaging was started about 45 min upon tumor cell application for in total $24 \mathrm{~h}$. Scale bar $=25 \mu \mathrm{m}$. 


\section{Lymphoid cells in breast to brain metastasis}

Since lymphocytes have been shown to infiltrate BrMs, the lymphoid compartment was analyzed during 99LN-BrM progression as well (figure 10). While the healthy brain parenchyma does not harbor any lymphoid cell types and $\mathrm{CD} 3^{+}$cells can only be attributed to border-associated areas (e.g. figure 14c), 99LN-BrM induces infiltration of $\mathrm{CD}^{+}$and $\mathrm{B} 220^{+}$cells, i.e. T and B cells, respectively (figure 10a, d). In accordance to the human situation (Harter et al., 2015), $\mathrm{CD}^{+}{ }^{+} \mathrm{TIL}$ infiltration was highest in smaller BrM, with a mean of approximately $5 \%$ of $\mathrm{CD} 45^{+}$cells, compared to larger BrM comprising approximately $2.4 \%$ (figure 10a,c). Contrary, the B cell population slightly increased during $\mathrm{BrM}$ progression, from an average of approximately $3 \%$ (small) to $4 \%$ (large) (figure 10a). Although not significant for both cell types, $\mathrm{CD}^{+}{ }^{+}$TILs trended to negatively correlate with BrM size, while the $\mathrm{B}$ cell population revealed a slight positive correlation with BrM growth (figure 10c). Analyzing the main $\mathrm{T}$ cell subsets in more detail revealed that both small and large $\mathrm{BrM}$ contained a similar relative $\mathrm{CD} 4^{+}: \mathrm{CD}^{+} \mathrm{T}$ cell ratio, which in addition was similar to the one from peripheral blood with a slightly higher percentage of $\mathrm{CD}^{+} \mathrm{T}$ cells (figure 10b). A very recent study showed similar results in human patient samples of carcinoma BrM (Friebel et al., 2020, Klemm et al., 2020). IHC staining confirmed the accumulation of $\mathrm{CD}^{+}$TILs within 99LN BrM (figure 10d).

Together, the data suggest a strong recruitment of especially $T$ lymphocytes at early stages of BrM establishment. In contrast to B lymphocytes, their number decreases during tumor progression. Alternatively the $\mathrm{CD}^{+}$lymphocyte population becomes thinned out during massive tumor growth. 
a

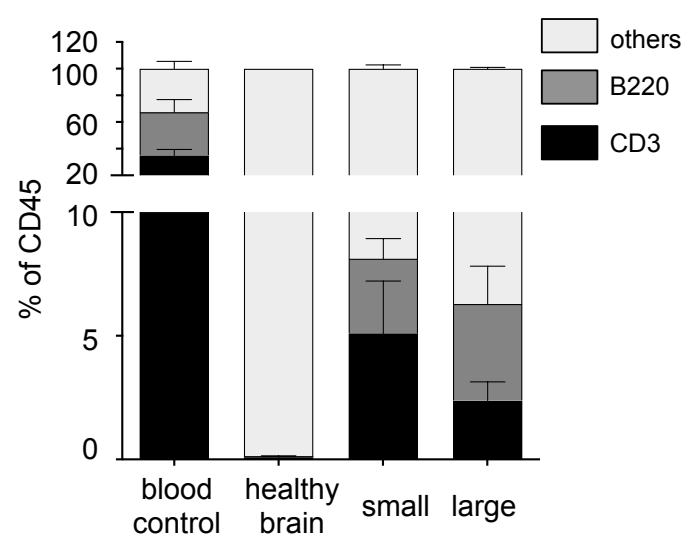

C

d

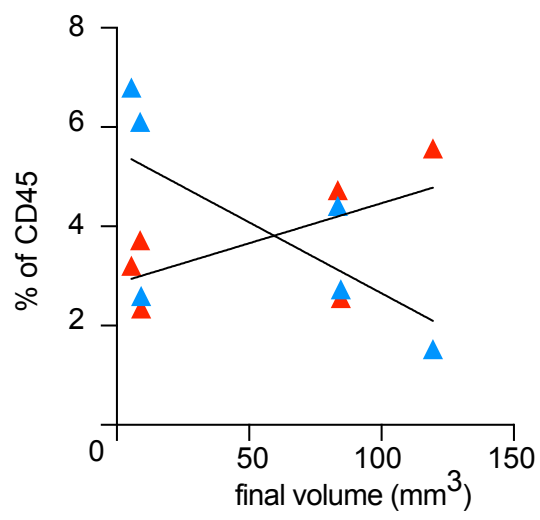

b

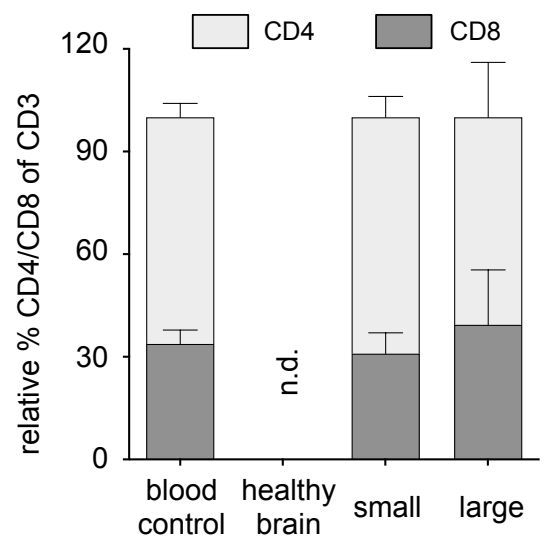

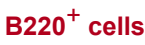

r: 0.64

p: 0.1711

$\mathrm{CD}^{+}$cells

r: -0.6787

p: 0.1383

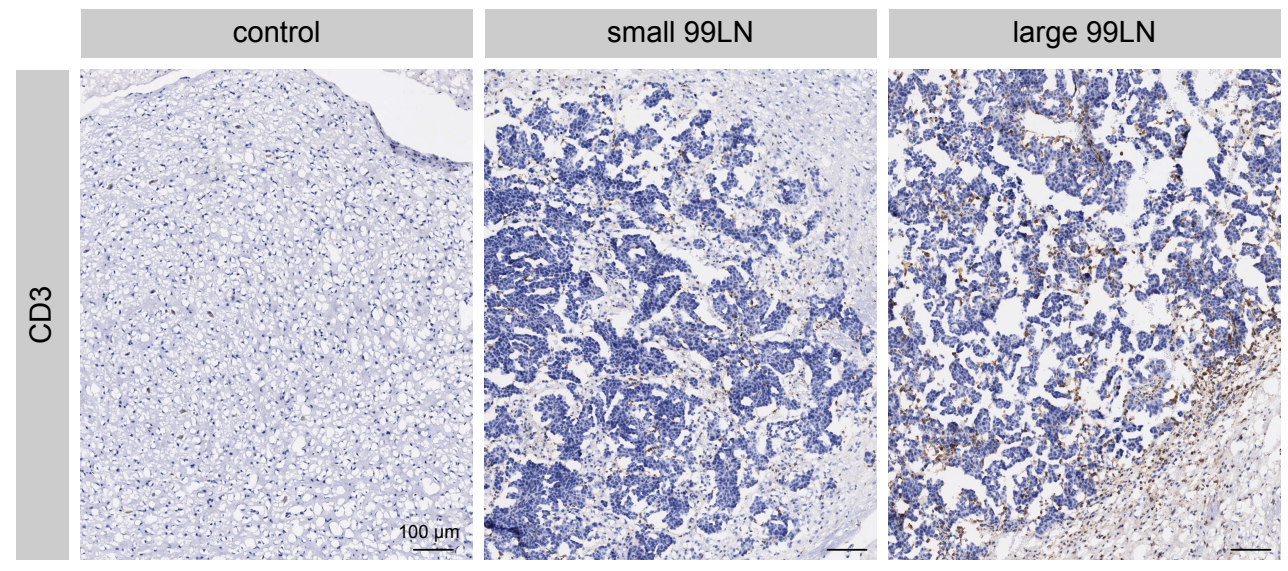

Figure 10: The lymphoid TME in 99LN breast-to-brain metastasis. a) Relative quantification of lymphoid cell populations in peripheral control blood, normal brain (control), and from small and large $99 \mathrm{LN}-\mathrm{BrMs}$, as determined by flow cytometry $(\mathrm{n}=$ 3 for every condition). For controls and BrM FCM data, full brains were used. b) Relative CD4-CD8 ratio as determined from subpopulations (as CD4 + CD8 = 100\%) of $\mathrm{CD}^{+}$cells in FCM. n.d. $=$not detected/ insufficient number. c) Percentage of $\mathrm{CD}^{+}$ (blue) or $\mathrm{B}^{2} 20^{+}$(red) cells among $\mathrm{CD} 45^{+}$cells correlated to final MRI-based BrM volumes. d) IHC staining for CD3 on a control brain slide, or from small and large 99LN BrM-containing mice. Scale bar $=100 \mu \mathrm{m}$. 
C) Whole brain radiotherapy (WBRT) affects the BrM TME in a dynamic manner

1. The primary tumor type drives changes in BrM-associated myeloid cell populations in response to WBRT

In order to understand the effects of standard of care therapy (WBRT) on the immune cell composition in different BrM models, tumor-bearing mice or control mice were irradiated with fractionated (5 × 2 Gy) or hypo-fractionated (1 $\times 10 \mathrm{~Gy})$ WBRT. Subsequently the TME was analyzed at different time points upon irradiation via FCM.

While the brains of non-tumor-bearing control Balb/c nude mice showed a small increase in their infl. monocyte (from mean of about $0.4 \%$ to about $1.3 \%$ ) and MDM (mean from $9.6 \%$ to $12.2 \%$ ) populations $3 \mathrm{~d}$ after the last dose of $5 \times 2 \mathrm{~Gy}$, this effect was transient and declined at d5 (inf. mono: 0.4\%, MDM: $11.3 \%$ ) (figure 11b). The granulocytic population lacked significant changes in response to WBRT and represented about $0.6 \%$ of $\mathrm{CD} 45^{+} / \mathrm{CD} 11 \mathrm{~b}^{+}$cells across all conditions (mean of mean) (figure 11b). Noteworthy, non-microglial cells detected within the controls are most likely not derived from the parenchyma, but rather from associated regions such as meninges that have not been removed during tissue harvest. For FCM of BrM, H1_DL2 or H2030 tissue with different final BLI values were macro-dissected at certain days upon WBRT (figure 11c). However, all mice from both models showed rather high radiance values. Within the melanoma model fractionated WBRT $(5 \times 2$ Gy) resulted in minor changes in the myeloid composition, but clearly induced the recruitment of more infl. monocytes and MDMs during the time course of $10 \mathrm{~d}$ following irradiation, compared to treatment-naïve BrM samples (figure 11d). However, relative MDM percentage increased from large untreated lesions (mean = $5.3 \%$ ) to irradiated lesions $10 \mathrm{~d}$ after $5 \times 2 \mathrm{~Gy}$ (mean $=9.2 \%$ ) (figure 11d). Contrary there was a continous recruitment of e.g. granulocytes and MDMs into H2030-BrM, which was even higher in response to fractionated WBRT (Schulz et al., 2020). Infiltration of granulocytes and infl. monocytes peaked at d5 after fractionated WBRT with a relative decrease of the granulocyte and infl. monocyte content at d10 after WBRT, due to an expansion of the total TAM population between d3 and d10 after WBRT (figure 11d) (Schulz et al., 2020).

Interestingly, the application of one single dose of $10 \mathrm{~Gy}$ WBRT resulted in diminution of the infl. monocyte and TAM-MDM populations in H1_DL2 at d3, and 
only affected TAM-MDMs in H2030, whose other BrM-associated myeloid populations revealed less prominent effects compared to d10 following $5 \times 2$ Gy at that time point (figure 11d) (Schulz et al., 2020). While the granulocyte and infl. monocyte populations remained similar at $\mathrm{d} 5 \mathrm{in} \mathrm{H} 2030$, especially infl. monocytes were repopulating H1_DL2 BrMs at $5 \mathrm{~d}$ upon $1 \times 10 \mathrm{~Gy}$. Similarly in both models however was that the TAM-MDM populations were initially reduced in response to $1 \mathrm{x}$ $10 \mathrm{~Gy}$, but rapidly recurred at d5. However, the extent of TAM-MDM repopulation differed between both models (figure 11d).

Together, the data revealed that fractionated WBRT regulates myeloid cell infiltration within xenograft BrM models in a tumor-specific manner and has the potential to enhance infiltration of cells upon initial depletion. Besides regulating cell population sizes, different WBRT regimens affect the morphology of BrM-associated TAMs (figure 12a), hence most likely they in addition induced the different expression of certain sets of genes in response to WBRT. BrM-infiltrated IBA $1^{+}$cells at d 5 upon $5 \mathrm{x}$ $2 \mathrm{~Gy}$ and at $\mathrm{d} 3$ upon $1 \times 10 \mathrm{~Gy}$ showed a more ramified phenotype than other conditions. Other brain-resident cell types (e.g. astrocytes, neurons) did not show any apparent morphological changes in response to fractionated or hypofractionated WBRT (figure 12b). 

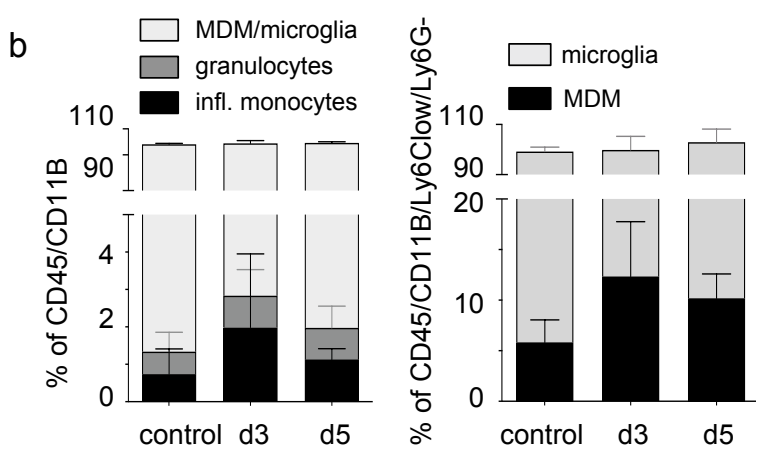

C
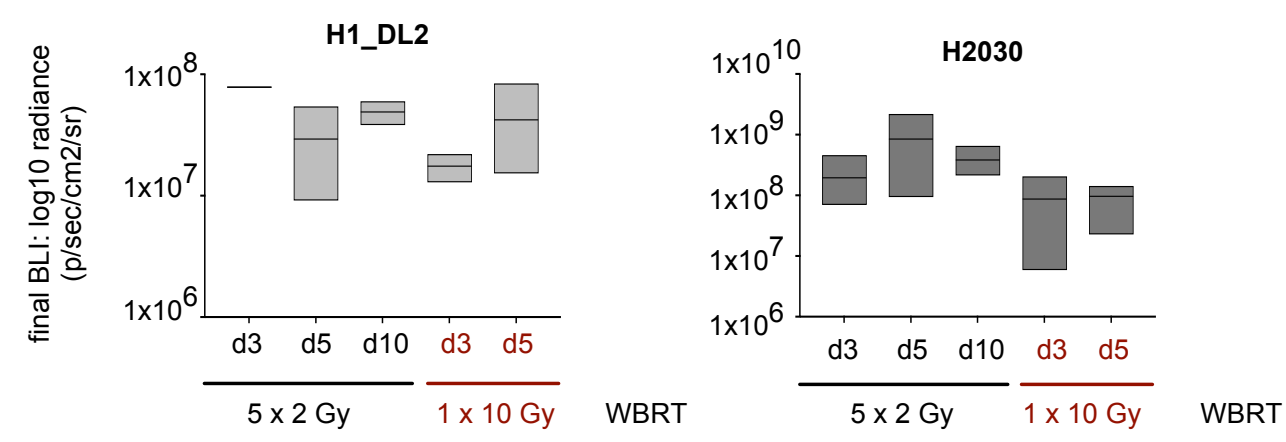

d
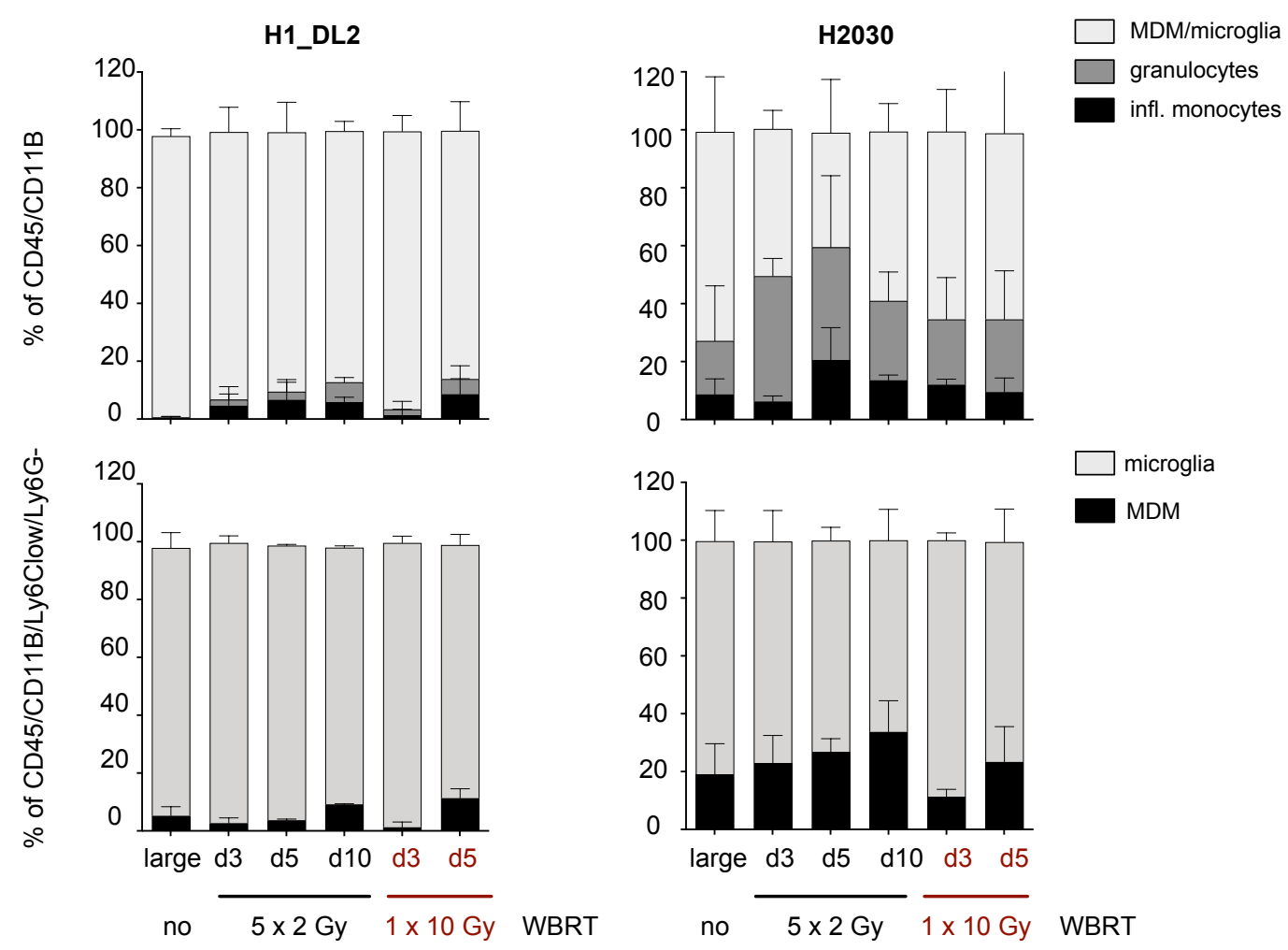

Figure 11: The dynamic BrM-associated myeloid immune cell compartment in response to WBRT. a) Schematic overview b) Relative quantification of myeloid cell populations in normal brain (control), and at 3 or $5 \mathrm{~d}$ upon WBRT from non-BrM bearing control mice. ( $n=3$ for every condition). Full brains were used for FCM. c) Final BrM values of WBRT-treated BrM-bearing mice used for FCM, which were stratified based on those values (as radiance). d) Relative quantification of myeloid cell populations in treatment-naive BrM (as control), and analyzed by FCM at several time points upon WBRT as indicated. ( $\mathrm{n}=3$ for every condition in every model, apart from $n=2$ in H1_DL2: d3 and d10 (5 x 2 Gy), and d3 (1 x 10 Gy)). Note that data derived from $\mathrm{H} 2030$ have been partially or completely published in Schulz et al., 2020. 


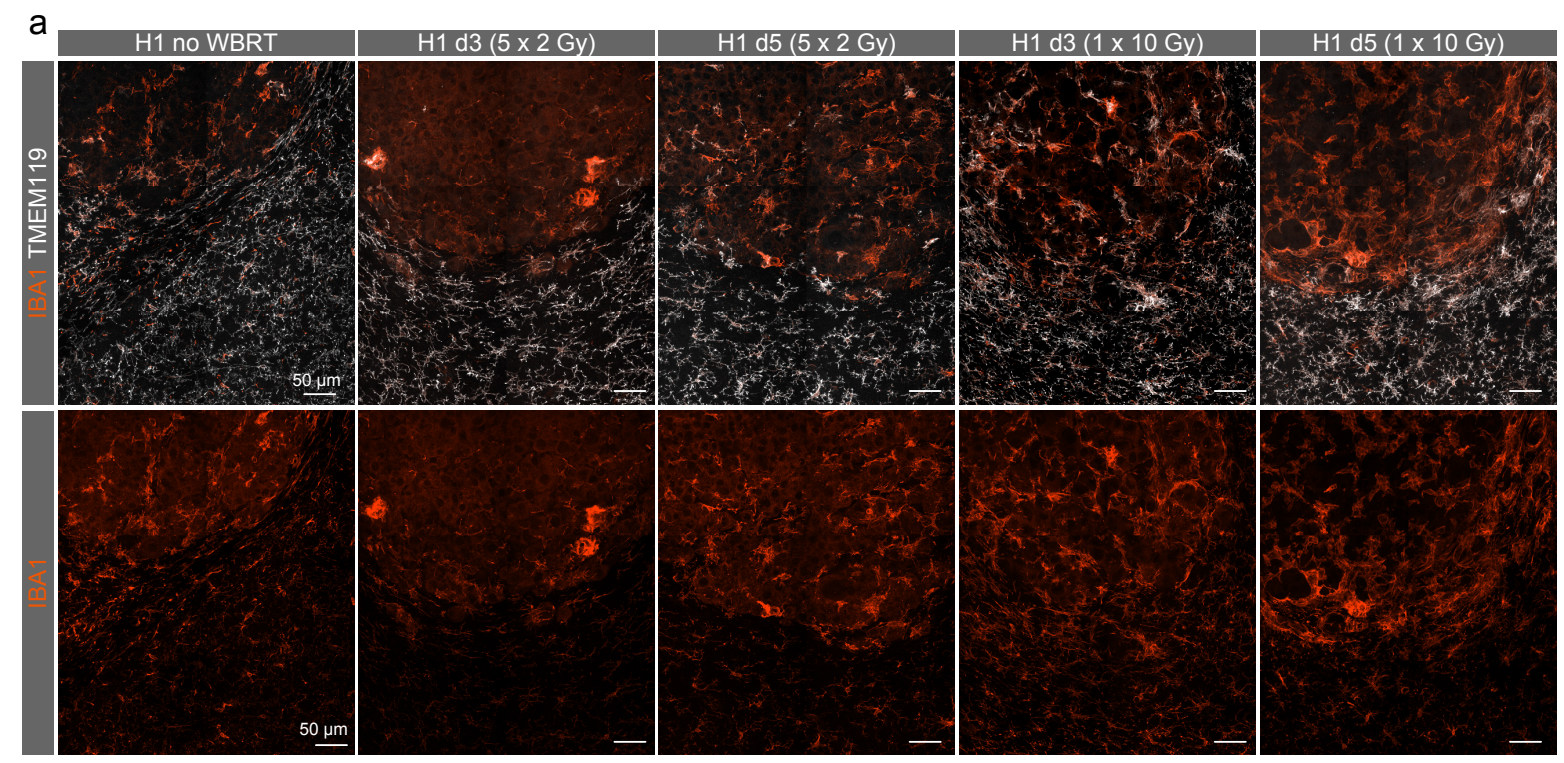

b

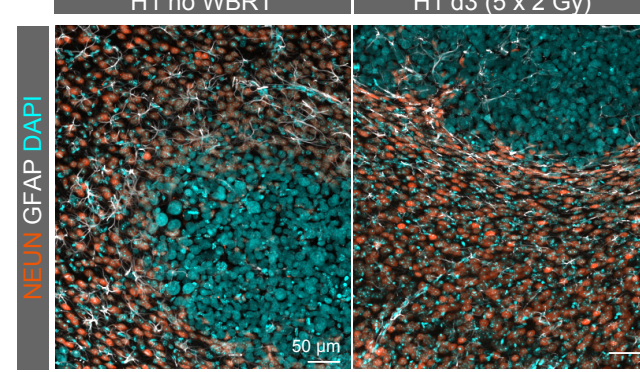

H1 d5 (5 x 2 Gy)

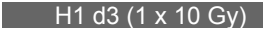

H1 d5 (1 x $10 \mathrm{~Gy})$
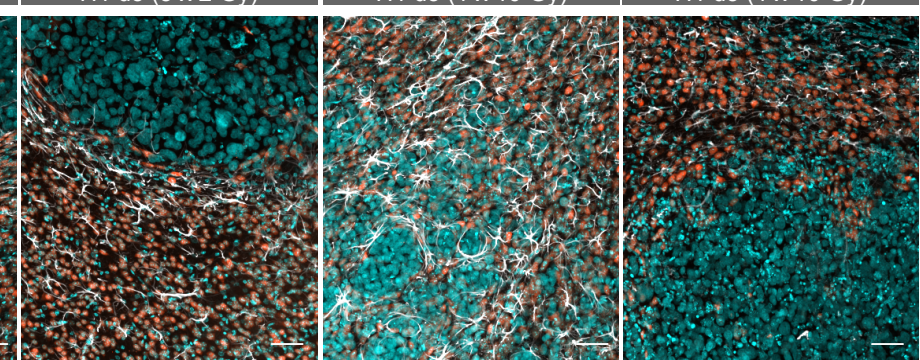

Figure 12: Morphologic changes in the TAM population in response to WBRT. a) Representative immunofluorescence double-stainings for IBA $1^{+}$(red) and TMEM $119^{+}$ (white) (upper panel), or IBA $1^{+}$only (lower panel) cells in treatment-naïve, or WBRTtreated H1_DL2 BrM. Scale bar $=50 \mu \mathrm{m}$. b) Representative immunofluorescence double-stainings for $\mathrm{NEUN}^{+}$(neurons) and $\mathrm{GFAP}^{+}$(astrocytes) associated to treatment-naïve or treated H1_DL2 BrM. DAPI (blue) was used as nuclear counterstain. Scale bar $=50 \mu \mathrm{m}$. 
2. Fractionated WBRT slows the recruitment of distinct myeloid cell populations in syngeneic breast-to-brain metastasis

To broaden the knowledge gained from FCM experiments in xenograft lung- and melanoma-to-brain metastasis models, 99LN-BrM-bearing (breast-to-brain) mice were analyzed upon receiving WBRT as well (figure 13a). Since the final BrM volume of WBRT-treated mice was rather low (figure 13b), untreated, small BrM samples were included in the comparison as treatment-naïve controls (figure 13c). While fractionated WBRT in non-BrM control mice reduced infl. monocyte and macrophage populations (most likely non-parenchymal) at $\mathrm{d} 3$ and $\mathrm{d} 5$ upon $5 \times 2 \mathrm{~Gy}$, two factors might influence the myeloid TME of 99LN BrM-bearing mice. First, WBRT disrupted an enhanced myeloid cell infiltration. The relative numbers of infl. monocytes and TAM-MDMs were very similar in WBRT-treated mice compared to treatment-naïve small 99LN samples. The infl. monocyte population in small lesions comprised approximately $3 \%$ of myeloid cells and remained similar $(2.72 \%)$ when analyzed at d5 following WBRT. Analogous, TAM-MDM numbers from small 99LN to WBRTtreated $99 \mathrm{LN}$ changed very slightly from $7.88 \%$ to $8.02 \%$ five days upon treatment (figure 13c). Furthermore, at d5 after WBRT the number of granulocytes slightly increased, and the overall trend across all cell types continued until at least $10 \mathrm{~d}$ upon fractionated WBRT (not shown). Secondly, and in addition it was identified that within that model hypo-fractionated (Chae et al., 2019), and even fractionated (Niesel et al., 2021) WBRT significantly slowed down BrM progression early after treatment, which most likely accompanies the lack of enhanced recruitment of WBRT-treated 99LN-BrM. Finally, to further visualize TAMs in treatment-naïve and treated BrM, IF of cleared $250 \mu \mathrm{m}$ thick brain sections was performed. While CD206 (or MRC1 mannose receptor c-type 1) under homeostatic conditions is associated to BAMs of the CNS (Goldmann et al., 2016) (figure 13d), neuro-inflammatory conditions recruit $\mathrm{CD}_{206^{+}} \mathrm{MDMs} / \mathrm{macrophages}$ (Jordao et al., 2019). Hence, in addition to the combinatory marker set of IBA1 and TMEM119, CD206 was included in subsequent IF staining. While control slices only showed IBA $1^{+} / \mathrm{CD} 206^{+}$cells at the border areas of the parenchyma, non-irradiated and irradiated 99LN BrM (figure 13d) induced the recruitment of IBA $1^{+} / \mathrm{CD} 206^{+}$cells (figure $13 \mathrm{~d}$ ). While IBA $1^{+} / \mathrm{TMEM} 119^{+}$cells could be localized in the periphery of $99 \mathrm{LN}$ BrM, a mixed population of IBA $1^{+} / T M E M 119^{\text {low }}$ and $\mathrm{IBA} 1^{+} / \mathrm{CD} 206^{+}$cells was found in a more infiltrative pattern (figure $13 \mathrm{~d}$ ), again 
suggesting that TAM-MGs align in the BrM periphery, while TAM-MDMs further infiltrate metastatic lesions.

a

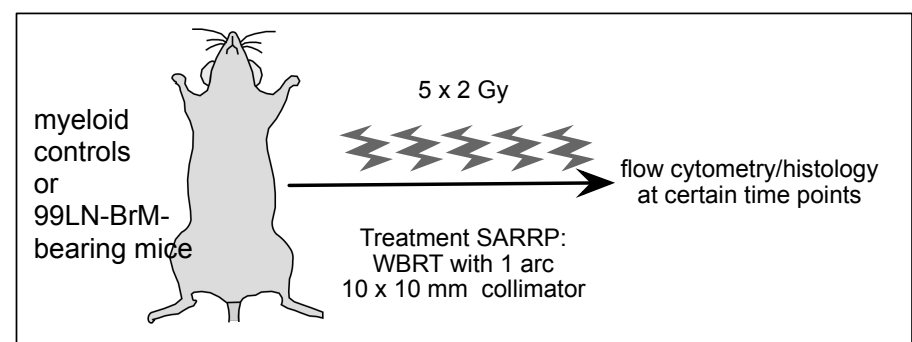

b

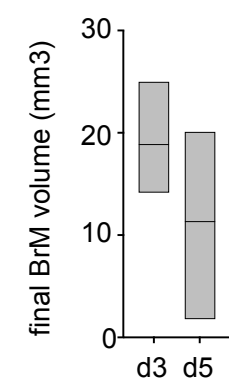

C
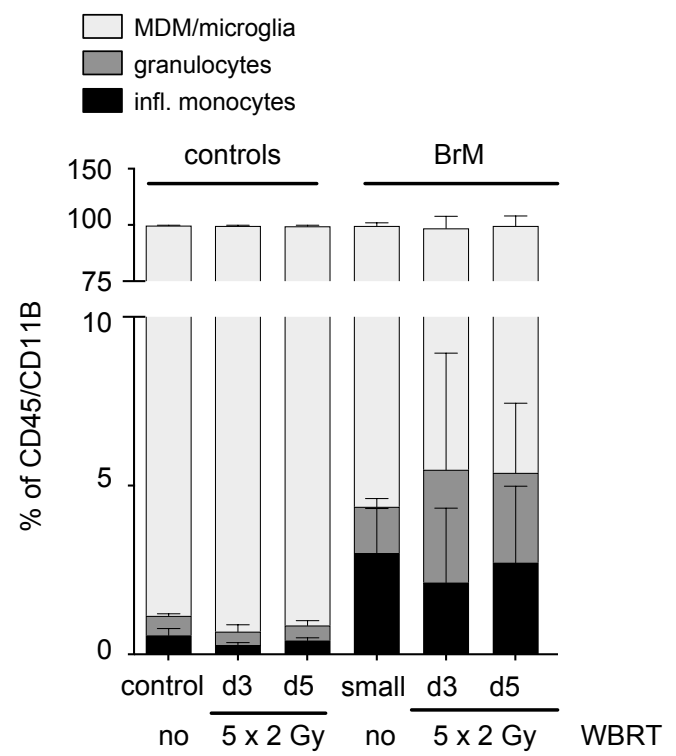

$\square$ microglia

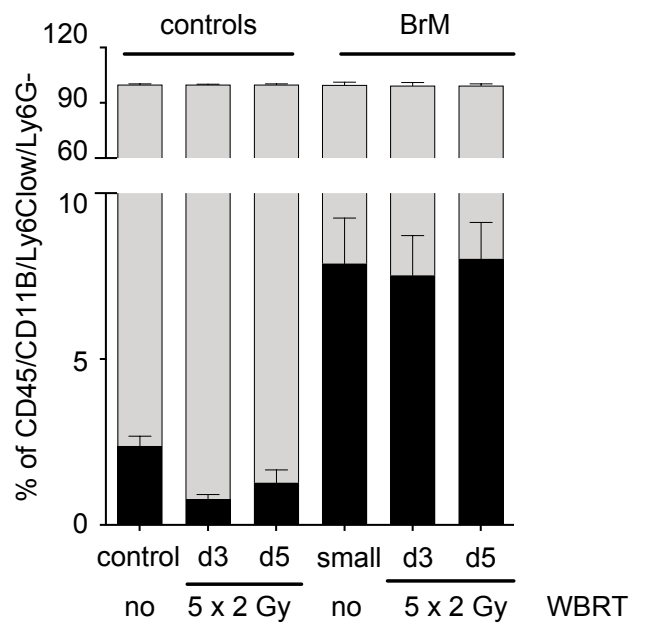

d

control

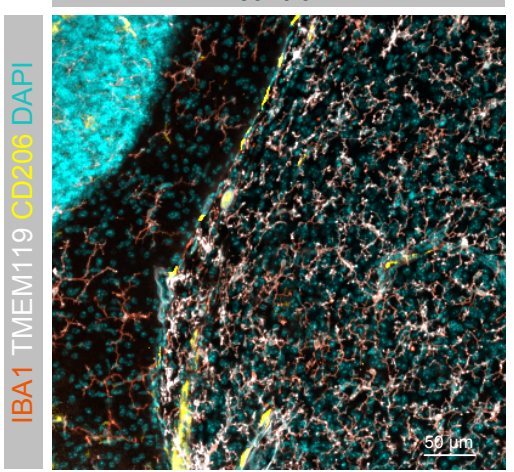

$\mathrm{BrM} / \mathrm{no}$ IR

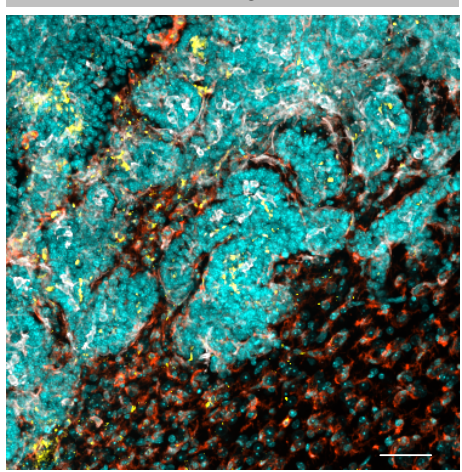

d5 / $5 \times 2$ Gy

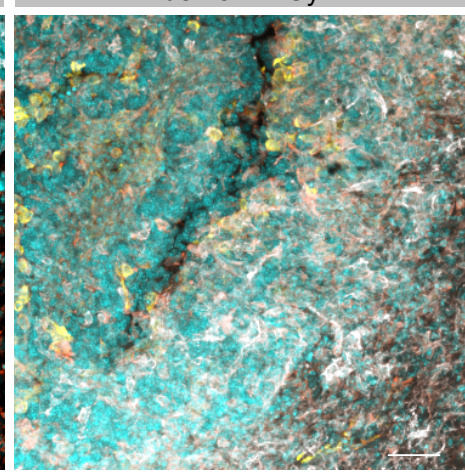

Figure 13: The breast cancer BrM-associated myeloid immune cell compartment in response to WBRT. a) Schematic overview b) Final BrM volumina based on MR image quantification, $\mathrm{d} 3$ and $\mathrm{d} 5: \mathrm{n}=3$. c) Relative quantification of myeloid cell populations in control brain (no BrM-no IR), and at d3 or d5 upon WBRT from nonBrM bearing control mice. ( $n=2$ for control, $n=3$ for IR controls). Full brains were used for FCM. The right part of each graph represents relative FCM data obtained from BrM samples either with or without WBRT, as indicated. $\mathrm{N}=3$ for samples related to BrM small, d3, and d5. d) Representative immunofluorescence triplestainings for IBA1 (red), TMEM119 (white), and CD206 (yellow) in control brain slice, 99LN-BrM without WBRT (middle), and at $5 d$ upon $5 \times 2$ Gy WBRT (right). DAPI (blue) was used as nuclear counterstain. Scale bar $=50 \mu \mathrm{m}$. 
3. WBRT changes the ratio of $\mathrm{CD} 4^{+}$and $\mathrm{CD} 8^{+} \mathrm{T}$ cells in 99LN-BrM

While recruitment of diverse myeloid cell populations seem to be influenced by WBRT, the effects of irradiation on the major lymphoid populations in BrM were examined within the 99LN BrM model (figure $14 \mathrm{a}$ ). The healthy brain parenchyma does not harbor lymphocytes (figure $10 \mathrm{a}, \mathrm{d}$ ), and the few associated lymphoid cells reside in border regions (figure $14 \mathrm{c}$ ). In contrast, BrM induces the recruitment of $B$ and $T$ cells. Apparently the influx of $T$ cells tended to negatively correlate with the size of 99LN-BrM. However, treatment applied as fractionated WBRT enhanced the influx of especially $\mathrm{CD}^{+}{ }^{+}$TILs into the parenchyma (figure 14b). Compared to small $\mathrm{BrM}$ (mean $=5.1 \%$ ), 99LN BrM at d5 upon $5 \times 2$ Gy contained approximately $8.43 \%$ $\mathrm{T}$ cells out of all $\mathrm{CD} 45^{+}$cells. Contrary, B lymphocyte numbers slightly decreased (from $3.02 \%$ to $2.45 \%$ ) in response to WBRT (figure 14b). Furthermore, both major lineages were detected in the metastatic tissue of thick brain sections via IF (figure $14 \mathrm{c}$ ). Very interestingly was the apparent change of relative $\mathrm{CD} 4^{+}: \mathrm{CD} 8^{+}$ratio within the $\mathrm{CD}^{+}$population (figure 14b). While the majority of $\mathrm{T}$ cells in treatment naïve $\mathrm{BrM}$ consisted of $\mathrm{CD}^{+}{ }^{+}$cells, WBRT reversed this ratio and already $3 \mathrm{~d}$ after $5 \times 2$ Gy the $T$ cell population consisted of more CD8 ${ }^{+}$TILs (figure 14b). Since WBRT in nontumor brains induced a certain amount of $T$ and $B$ cell recruitment, and the $T$ cell population consisted of relatively more $\mathrm{CD}^{+} \mathrm{T}$ cells, this effect seen in BrM might be driven by irradiation itself rather than the presence of $\mathrm{BrM}$.

Together the data revealed that fractionated WBRT enhanced $\mathrm{CD} 3^{+} \mathrm{TIL}$ infiltration in breast-to-brain metastasis (99LN) at early time points following WBRT. However little is known about the effects of tumor cells on the transcriptional landscape of TILs. 
a

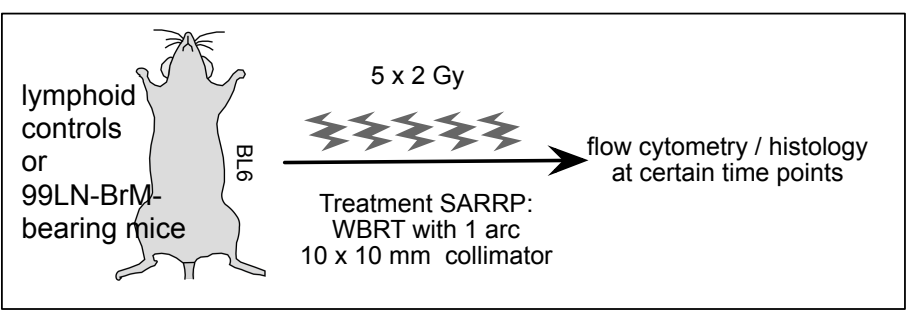

b
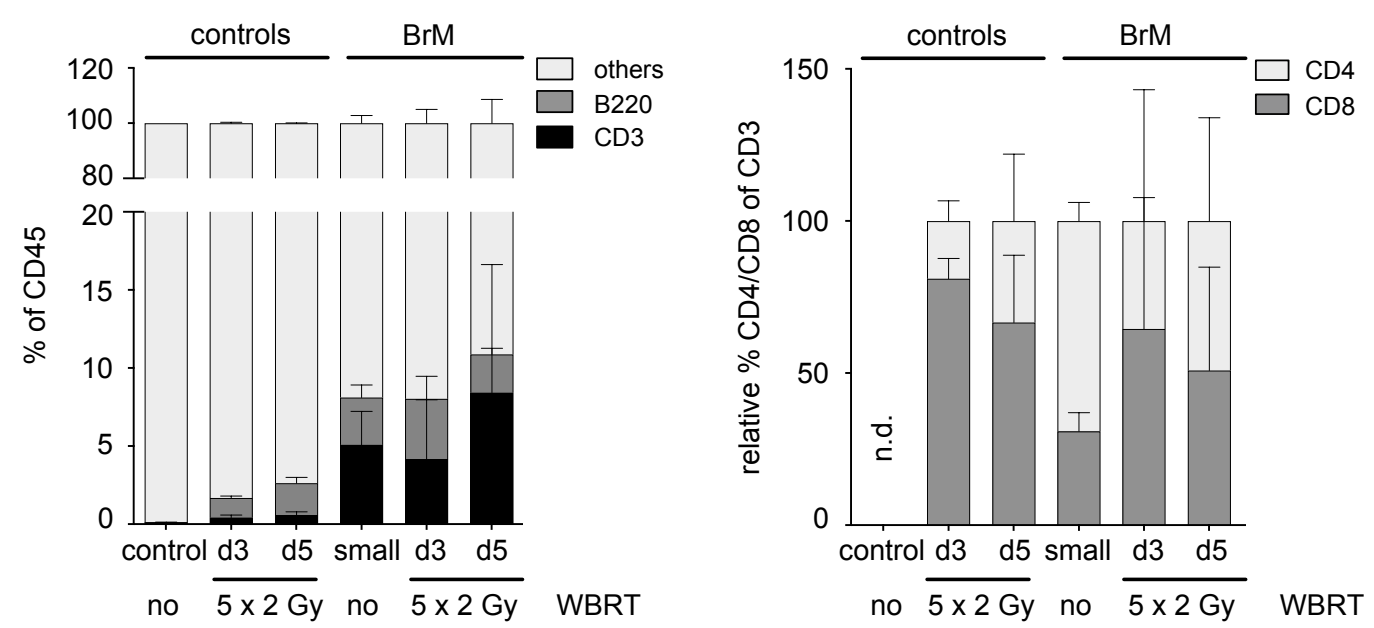

C
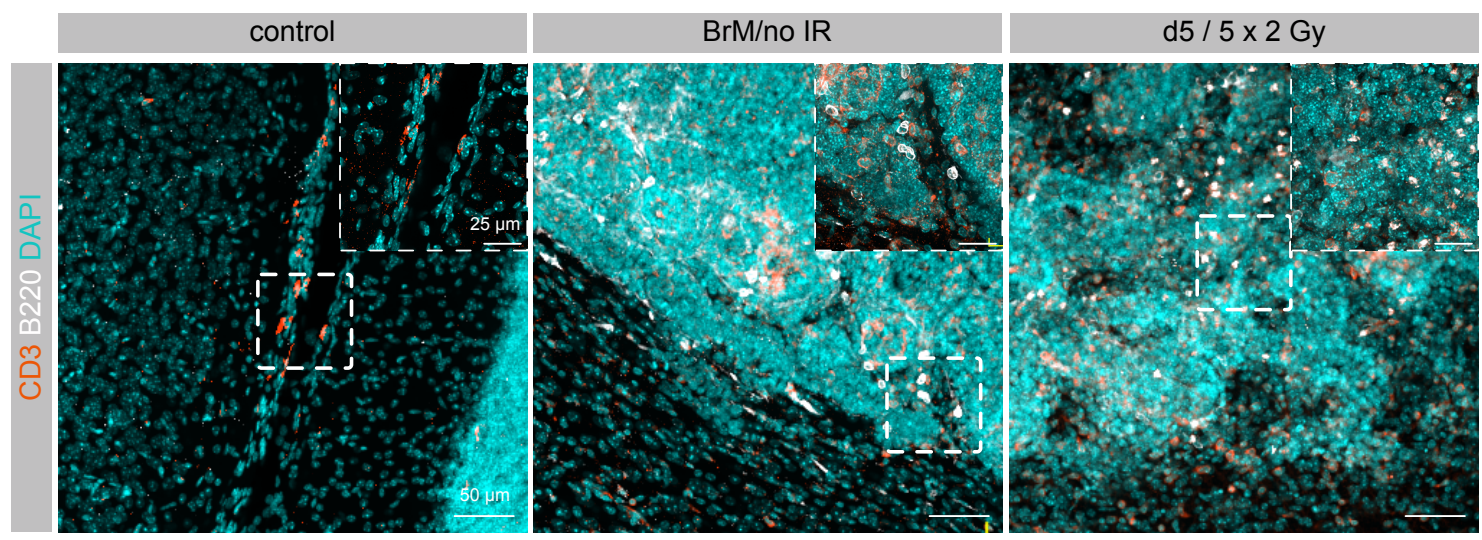

Figure 14: The breast cancer BrM-associated lymphoid immune cell compartment in response to WBRT. a) Schematic overview b) Relative quantification of lymphoid cell populations in control brain (no BrM-no IR), and at d3 or d5 upon WBRT from nonBrM bearing control mice. ( $n=2$ for control, $n=3$ for IR controls). Full brains were used for FCM. The right part of each graph represents relative FCM data obtained from BrM samples either with or without WBRT, as indicated. The right graph represents the relative distribution of $\mathrm{CD}^{+}$or $\mathrm{CD}^{+}$cells among the $\mathrm{CD}^{+}$cell population. For plotting, the amount of $\mathrm{CD}^{+}$plus $\mathrm{CD}^{+}$cells was set as $100 \%$, and the relative ratio calculated. $\mathrm{N}=3$ for samples related to small, $\mathrm{d} 3$, and $\mathrm{d} 5$. $\mathbf{c}$ ) Representative immunofluorescence double-stainings for CD3 (red) and B220 (white) in control brain slice, 99LN-BrM without WBRT (middle), and at $5 \mathrm{~d}$ upon $5 \times 2$ Gy WBRT (right). DAPI (blue) was used as nuclear counterstain. Scale bar $=50 \mu \mathrm{m}$. 
D) RNA-Sequencing reveals molecular responses in BrM-associated immune cells

Upon investigation of cellular changes following BrM induction, progression, and in response to WBRT, molecular changes associated to BrM-induced inflammatory responses were examined via RNA-Sequencing (RNA-Seq) of sorted immune cells across different models.

\section{TILs in 99LN-BrM show an activated/exhausted phenotype}

To elucidate transcriptional traits of 99LN-associated TILs, RNA-Seq experiments of $\mathrm{CD}^{+}, \mathrm{CD}^{+}$, and $\mathrm{B} 220^{+}$cells purified from treatment-naïve 99LN-BrM (figure 15a,b) were performed. $\mathrm{CD} 4^{+}, \mathrm{CD}^{+}$, and $\mathrm{B}_{22} 20^{+}$cells from peripheral blood of WT mice were used as controls. The purity of sorted cells was assessed by IF prior to RNASeq (figure 15b), as well as post-Seq via lineage-specific marker detection (figure $15 \mathrm{~d})$. Representation of normalized counts yielded an expression pattern as expected, wherein the T cell subsets expressed major lineage markers CD4 or CD8 at higher levels than B cells. Similarly, B cell specific marker expression of CD19 and CD20 was highest to $B$ cells in both, the control and BrM situation (figure 15d). Overall, unsupervised principal component analysis (PCA) of control vs. TIL samples showed that the highest variance across all samples can be explained by cell state (control or BrM-associated), whereas the second highest variance was cell typederived ( $T$ cell or $B$ cell) (figure $15 \mathrm{c}$ ). Dissecting the transcriptional changes in more detail revealed a variety of differentially expressed genes (DEGs) across the three cell types (figure 15e), either up- or downregulated comparing control vs. BrMassociated cells. While the majority of these DEGs was cell type-specific, a certain overlap existed in particular between $\mathrm{CD}^{+}$and $\mathrm{CD}^{+}$cells (figure 15e). Additionally there were 106 DEGs commonly up-, and 116 DEGs commonly downregulated across all three cell types. Among the upregulated genes across all cell types were Ccr2, Ccr5, Ccr8, Cln8, Ctla4, Cxcr3, Fos, Gzmb, Gzmk, II2Ra, Irf8, Lag3, P2rx7, Pdgfb, Sema6d, Tcrg-C2, and Xcl1. Commonly downregulated genes included Aldh2, CD9, CD81, F5, Gp5, Gp6, Gp9, Itga6, Pecam1, Pf4, Snca, and Vwf (figure 15e).

Although $\mathrm{T}$ cells accumulate in $99 \mathrm{LN}$ BrM, there seems no sustainable anti-tumor effects. Hence, the expression of distinct activation/exhaustion marker of $\mathrm{CD}^{+}$and CD $8^{+}$TILs was examined (figure 15f). There was a significant upregulation of Ctla4, 
Gzmk, Helios, Ifng, Lag3, and Tnf in both lineages, as well as a significant upregulation of PD1 in CD8-TILs (figure 15f). Although not significant, the specific regulator of $\mathrm{T}$ cell activation Tox was slightly upregulated in $\mathrm{CD}^{+}$, but especially $\mathrm{CD}^{+}$TILs. To get insight into pathways and putative functions associated to all DEGs in $\mathrm{CD}^{+}$and CD8 ${ }^{+}$TILs, pathway analyses were performed (figure $15 \mathrm{~g}$ ). While CD4 ${ }^{+}$TILs upregulated genes belonging to "cytokine-cytokine receptor interaction", "positive regulation of cell migration", "inflammatory responses" or "leukocyte differentiation", CD8 ${ }^{+}$TILs showed higher levels of transcripts related to "cell cycle" and "mitosis" (figure 15g). Noteworthy, both cell types downregulated genes related to "coagulation", "clotting", and interaction with the local environment (e.g. "ECMreceptor interaction", "cell substrate adhesion"), suggesting profound molecular changes in TILs upon entry into the CNS parenchyma.

Very interesting was the B cell population associated to BrM (TIL-B cells), which upregulated certain genes usually related to T cells (figure 15h). Among them were $C D 8 a$ and $C D 8 b 1$, for instance. Furthermore, there was an upregulation of certain other cytokines (e.g. Cxcl10, Gzmb), or cytokine receptors (Ccr9, Tnfrsf9) within the Top 50 DEGs, which one usually would assume to find in T cells. Downregulated in TIL B cells were Cnn3, which encodes calponin 3, a protein implicated in mature B cells and BCR signaling (Flemming et al., 2015, Shi et al., 2015), or the complement regulating factor CD55. Functional annotation of TIL B cell-associated DEGs suggests an activated, yet impaired phenotype (figure 15i). Several DEGs related to $B$ cell receptor signaling were downregulated in TIL B cells.

Together, the data reveal that TILs in BrM show rather over-activated/exhaustive phenotypes in both lineages $\left(\mathrm{CD}^{+} / \mathrm{CD} 8^{+}\right)$, as frequently observed in solid tumors (Davoodzadeh Gholami et al., 2017). In addition, data of B cells associated to 99LN further suggest a weakened activity. Hence, it was tempting to examine the myeloid compartment in that model in more detail, since this one is among other cell types associated to immune-suppressive functions. 
a

b

bulk RNA Seq upon macro dissection

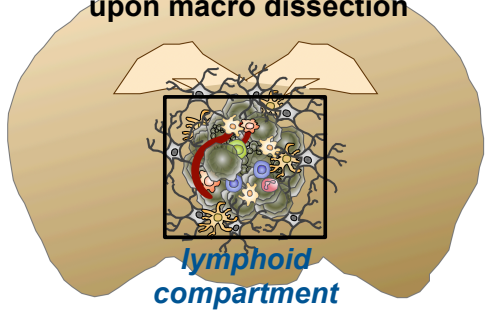

d

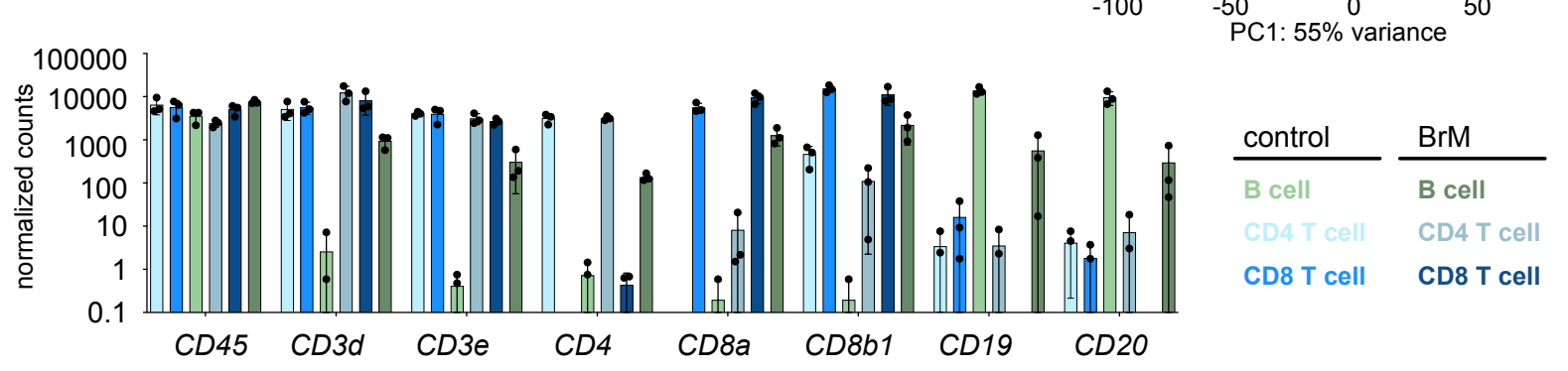

e

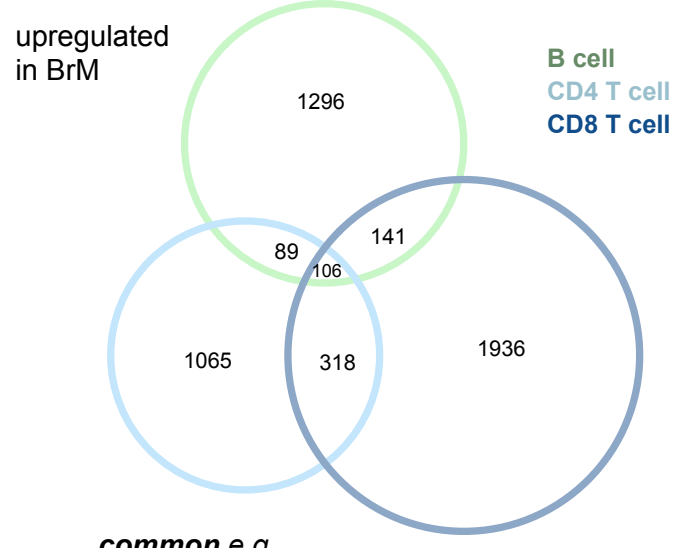

Ccr2, Ccr5, Ccr8, Cln8, Ctla4, Cxcr3,

Fos, Gzmb, Gzmk, II2Ra, Irf8, Lag3,

P2rx7, Pdgfb, Sema6d, Tcrg-C2, Xcl1

downregulated in BrM

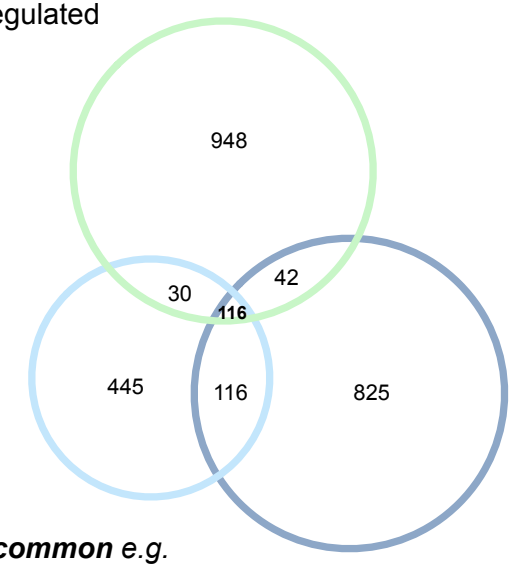

Aldh2, CD9, CD81, F5, Gp5, Gp6,

Gp9, Itga6, Pecam1, Pf4, Snca, Vwf
C

sorted from $99 \mathrm{LN}-\mathrm{BrM}$
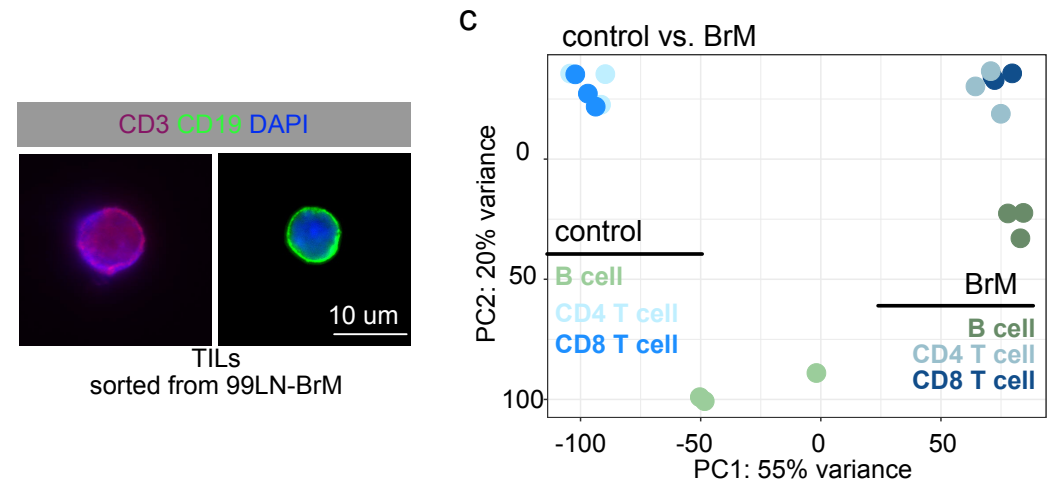

f

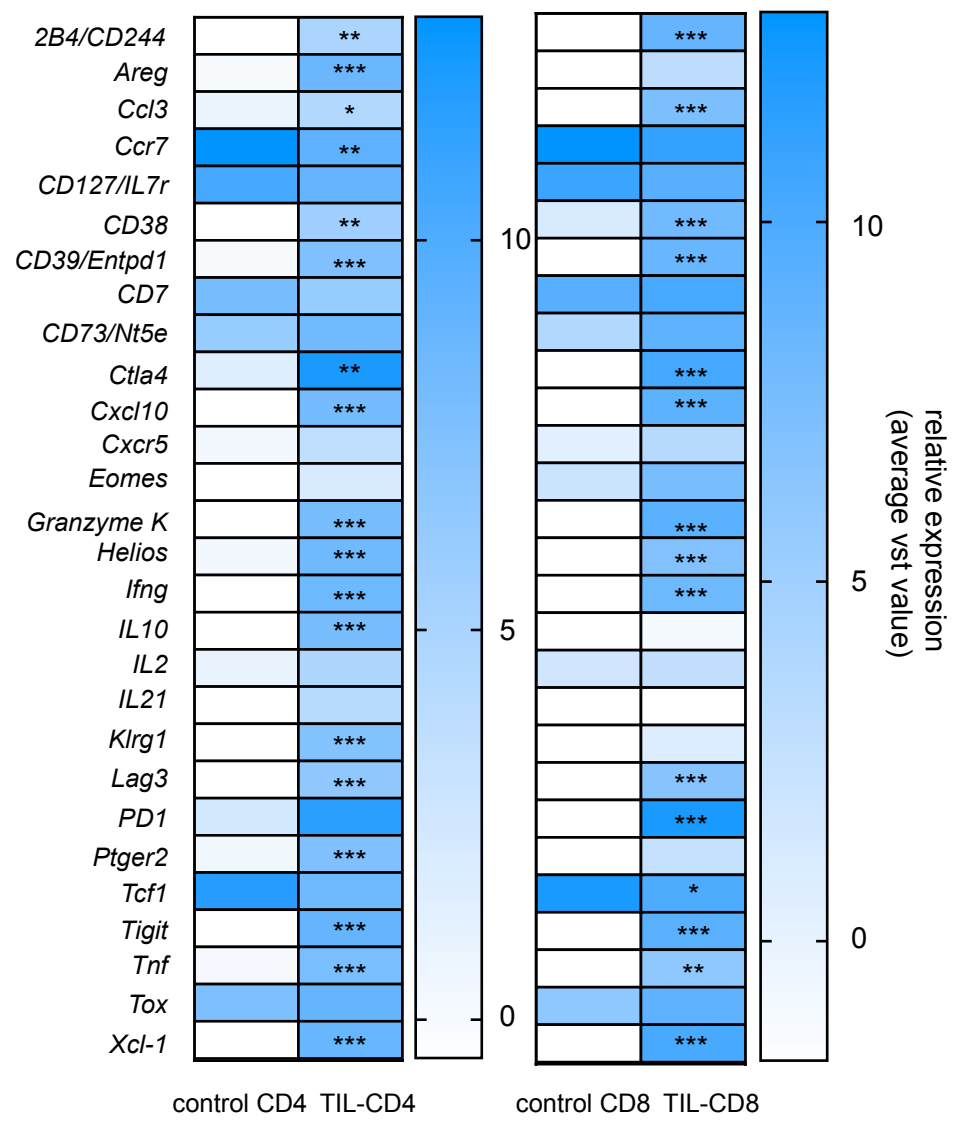



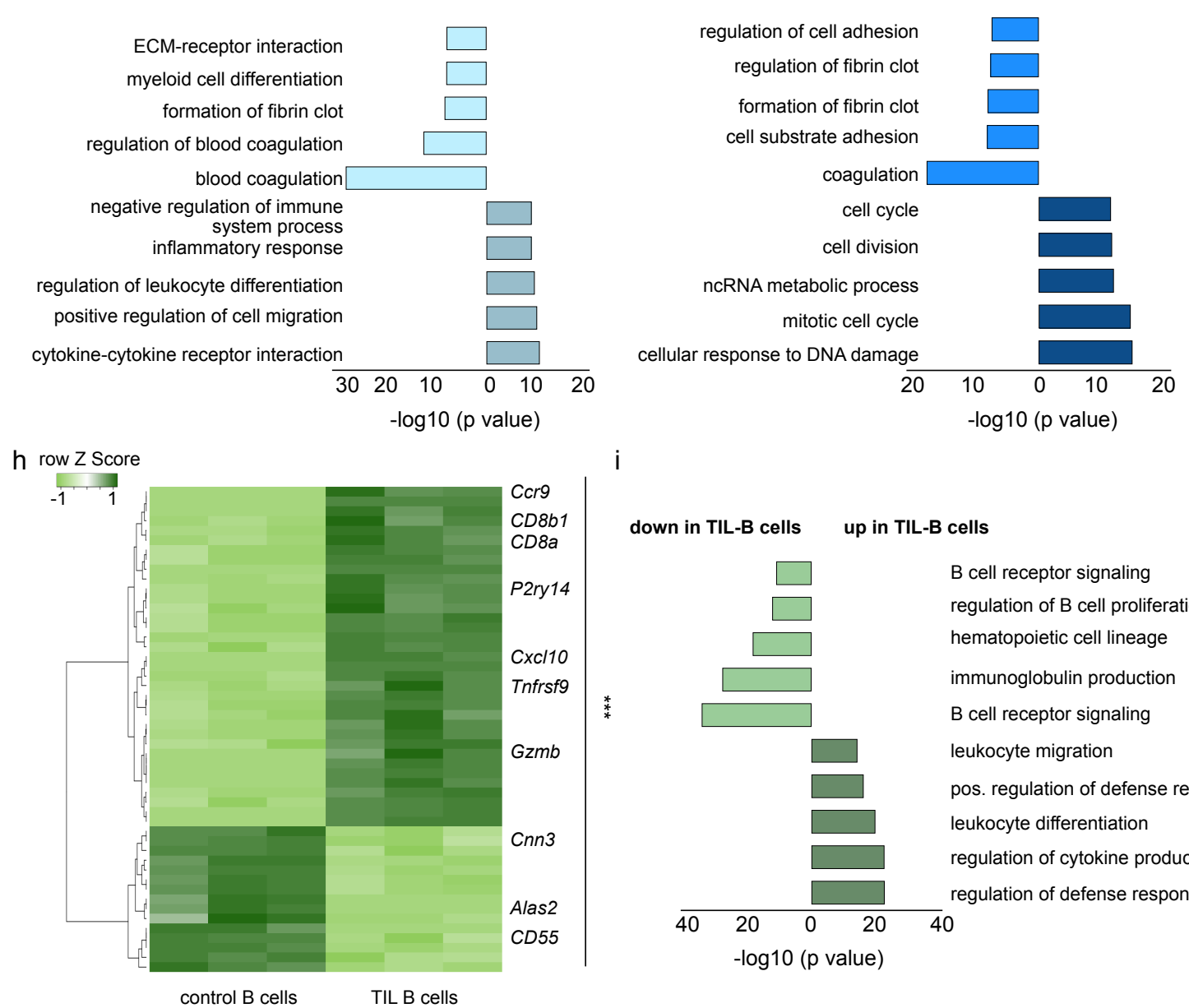

i

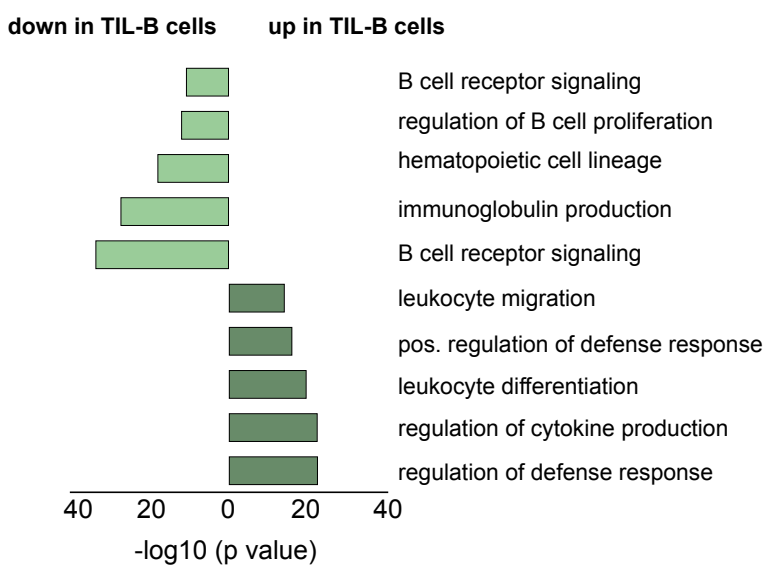

Figure 15: 99LN-BrM-associated lymphoid cells upregulate markers of activation. a) Schematic overview. b) Immuno-fluorescence staining of CD3 (red) and CD19 (green) lymphoid cells sorted out of 99LN-BrM. DAPI (blue) was used as nuclear counterstain. Scale bar $=10 \mu \mathrm{m}$. c) Principle component analysis (PCA) of control vs. BrM-associated lymphoid cell types. $n=3$ for each condition. d) Normalized Counts of selected genes related to major lymphoid lineages. Data are represented as bar graphs with individual values as dots. e) Euler Plots representing unique and overlapping DEGs for both, up (top) and down (bottom) regulated genes of the three major lymphoid populations of interest, which were selected based on basemean > 20 and padj. value $<0.05$. f) Heatmap of selected T cell-specific activation marker, data derived from single cell type comparisons (ctrls. vs. BrM) in $\mathrm{CD}^{+}$and $\mathrm{CD}^{+} \mathrm{T}^{\mathrm{T}}$ cells. Data are represented as average vst value/per group. Significance derived from padj. values with: ${ }^{*} p<0.05,{ }^{* *} p<0.01,{ }^{* * *} p<0.001$. g) Pathway analysis of the Top 5 up or downregulated pathways. Data derived from subjecting significant DEGs from ctrl. vs. BrM-associated cell type to Metascape analysis based on positive log2FC (upregulated) or negative log2FC (downregulated). h) Heatmap of Top 50 DEGs within the B cell compartment of 99LN-BrM, comparing TIL-B cells with their respective normal cellular counterpart (control). All up- and downregulated genes within each group of comparisons are highly significant (padj $<0.001,{ }^{* * *}$ ). Certain gene names are indicated. i) Pathway analysis of the Top 5 up or downregulated pathways from B cells. Data derived from subjecting significant DEGs from ctrl. vs. BrM-associated cell type to Metascape analysis based on positive log2FC (upregulated) or negative log2FC (downregulated). Data deposited at GSE164049 and associated to Niesel et al., 2021. 
2. 99LN-BrM infiltrating myeloid cells upregulate marker to interact with TILs In order to analyze the effects of the presence of TAMs and TILs within BrM, RNASeq of the major TAM populations (i.e. TAM-MGs, TAM-MDMs, and TA-Monos) associated to 99LN-BrM was performed (figure 16a) (published in Niesel et al., 2021). As controls MG from normal, healthy mice as well as monocytes from peripheral blood were used. The quality of the sorting strategy was initially determined by qPCR (not shown), and verified post-Seq based on normalized counts of lineage specific markers (figure 16b). These marker expression confirmed lineage identity of the sorted cell populations and showed higher expression levels of typical MG markers (e.g. Cx3cr1, P2ry12, and Tmem119) in MG, and markers related to peripheral macrophages (e.g. Ccr2, Itga4 (= CD49d), and Runx2) higher expressed in blood monocytes, TA-Monos and TAM-MDMs. Examining transcriptomic profiles more globally across all controls vs. all TAM populations revealed a strict clustering according to cell type (PC1) and condition (PC2, control vs. BrM) (figure 16c), similar as seen in the lymphoid compartment (figure 15c). Analyzing all significant DEGs revealed a high set of unique and overlapping up- and downregulated genes between the three cell types. Commonly upregulated was a set of 372 genes, and 269 genes were downregulated in all lineages (figure 16d). The upregulated DEGs included Apoe, Axl, B2m, C4b, C5ar1, Cc/2/4/7/8/12, CD72, CD83, Cdk1, Cish, Cldn1, Ctsb/c/d/h/l/z, Cx3cl1, Epcam, Gas6, Igf1, Il1a, II1b, Irf7, Kit, Ldha, Ltf, Mif, Mmp14, P2rx4, P2ry14, Serinc2, SIc2a1, Socs2, Socs3, Tgfbi, Timp2, TIr1, TIr2, Trf, Vcam1, Wfdc2, and Wfdc18. Significantly downregulated across all three cell types were Akna, Atm, Birc6, Celf2, Cop1, Dhx9, Dock8, Evi5, Foxp1, Git2, Herc1, Itch, Klf7, Lyst, Nfkb1, Ogt, Phc3, Pygm, Rock2, Smarca2, Son, Srsf5, Syk, Tia1, Xiap, Xist, and Zfp84 (figure 16d). While TA-Monos regulated about 800 DEGs in both directions, TAM-MDM gene regulation was much higher, with about 1.400 and 1.300 DEGs up-, or downregulated, respectively. This analysis revealed an apparent overlap between TA-Monos and TAM-MDM (figure 16d), similar to TAMs of the H2030-BrM model (figure 17, Schulz et al., 2020). Moreover, there were about 200 DEGs each, similarly regulated between both major TAM populations, while TAMMGs showed a tremendous regulation of approximately 2.100 DEGs in each direction (figure 16d). Semi-supervised hierarchical clustering of the Top 50 DEGs in both TAM-MG and TAM-MDM further revealed the unique and only partially overlapping character among the most highly differently expressed genes compared 
to their normal cellular counterparts (figure 16e). TAM-MG upregulated markers related to interferon signaling (IIf7, Zbp1), the DAM marker Apoe, or complement (C4b) for example (figure 16e). Downregulated in TAM-MG was for instance Cst3, a marker which is attributed to homeostatic MGs across species (Keren-Shaul et al., 2017, Geirsdottir et al., 2019). The other phagocyte population (TAM-MDM) showed an upregulation of several genes related to cytokine signaling (Cc/2, Cc/8, Ccr5), complement members (e.g. C3ar1), but also an upregulation of markers, which are associated to interaction with the adaptive immune compartment (e.g. CD72, CD83) (figure 16e). To also visualize a physical interaction of $\mathrm{CD}^{+}$cells and TAM-MDMs, immuno-fluorescence double staining for CD3 and CD206 was performed on thick sections. Staining confirmed a close proximity of both cell types in the 99LN-BrM TME (figure 16f). Although RNA-Seq data are derived from bulk populations, relative expression levels of distinct markers of the TAM compartment, which are relevant for the interaction between TAMs and TILs were further examined. Surprisingly, neither $P D-L 1$ (CD274) nor PD-L2 were significantly upregulated on either TAM population, and remained at rather lower expression levels (figure 16g). While CD80 and CD86 were downregulated on TAM-MG, CD80 remained unchanged, but CD86 showed significantly higher expression levels on TAM-MDM (figure 16g). Further marker (Icosl, B7-H3) critical for the interaction between antigen-presenting cells (APC, like macrophages) and $\mathrm{T}$ cells, showed strong (Icos/) or highly significant (B7-H3) upregulation on especially TAM-MDMs. Although expression levels remained relatively high, Vsir, a gene encoding for the negative immune checkpoint regulator VISTA, was downregulated in both cell types (figure 16g). Furthermore, there was an upregulation of two other markers related to $\mathrm{T}$ cell interaction (CD40, Tnfsf9), altogether suggesting that there is interaction between the myeloid and lymphoid compartment within 99LN-BrM. In addition, genes related to antigen-presentation type II (H2-Aa, H2-Ab1, H2-Oa) were highly significantly upregulated on TAM-MDMs (all), or TAM-MG (H2-Aa, H2-Ab1), but all of them at much higher levels in MDMs. Contrary, H2-D1, a gene associated to MHC I-mediated antigen-presentation was highly significantly upregulated in TAM-MGs (figure $16 \mathrm{~g}$ ).

In summary, transcriptomic data derived from the myeloid compartment associated to the breast-to-brain metastasis model 99LN suggest the establishment of a dynamic environment, which in conjunction with lymphoid RNA-Seq data point towards an immune-suppressive milieu. While interactions between MDMs and T cells are likely 
to occur, they probably also include antigen-presentation, in sum leading to TIL expansion indicative of activation, followed by TIL exhaustion as further described in Niesel et al. (2021).

a

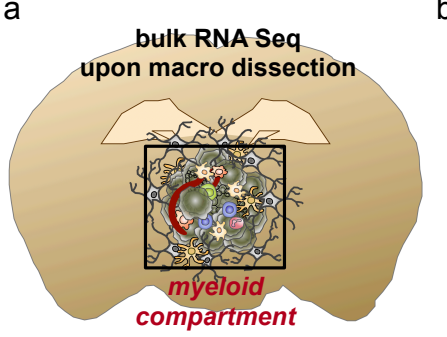

C

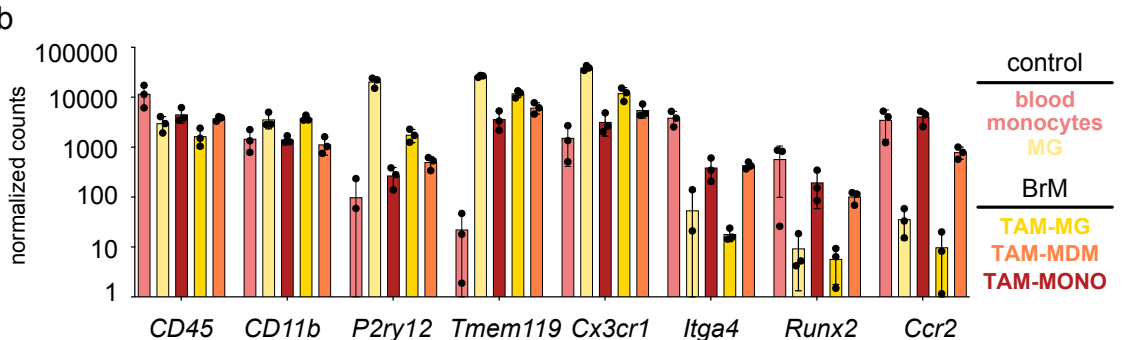

d

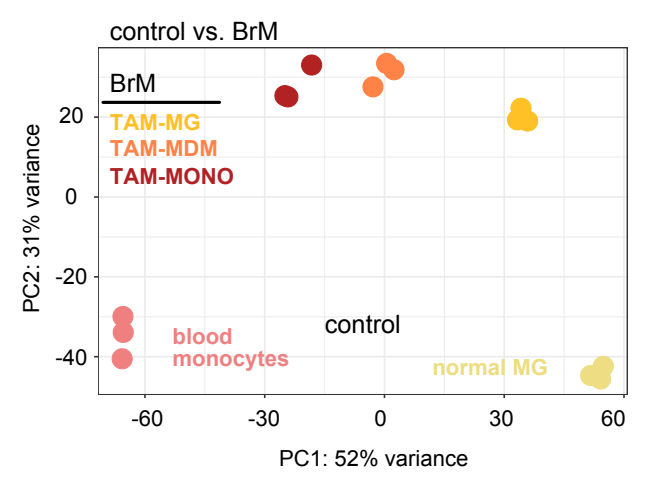

e g

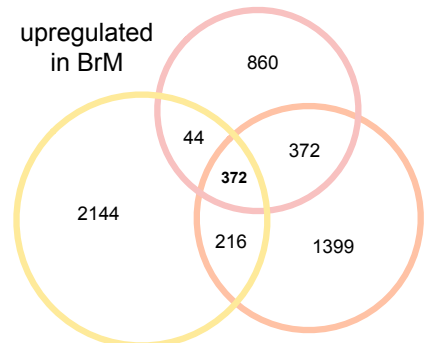

common e.g. Apoe, Axl, B2m, C4b, C5ar1

Ccl2/4/7/8/12, CD72, CD83, Cdk1, Cish, Cldn1, Ctsb/c/d/h/l/z, Cx3cl1, Epcam, Gas6, Igf1, I/1a, II1b, Irf7, Kit, Ldha, Ltf, Mif, Mmp14, P2rx4, P2ry14, Serinc2, Slc2a1, Socs2, Socs3, Tgfbi, Timp2, TIr1, TIr2, Trf, Vcam1, Wfdc2, Wfdc18

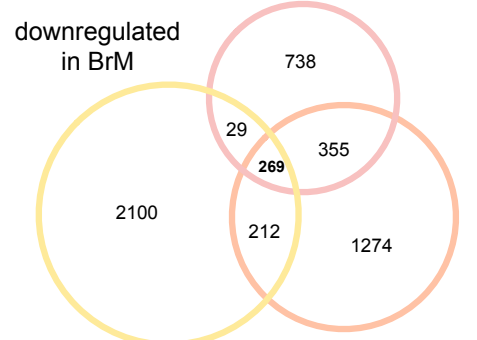

common e.g. Akna, Atm, Birc6, Celf2, Cop1, Dhx9, Dock8, Evi5, Foxp1, Git2, Herc1, Itch, KIf7, Lyst, Nfkb1, Ogt, Phc3, Pygm, Rock2, Smarca2, Son, Srsf5, Syk, Tia1, Xiap, Xist, Zfp84
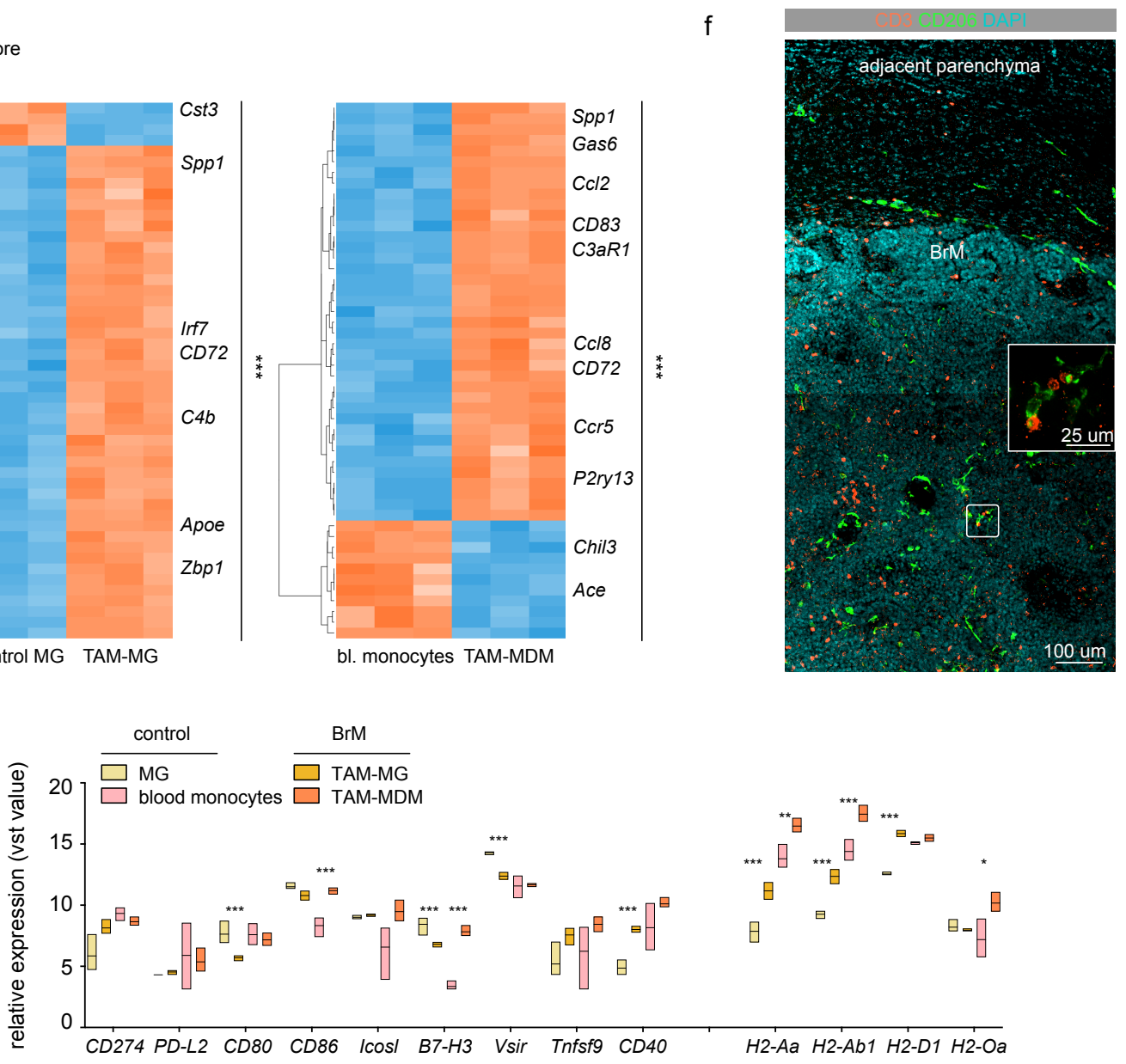
Figure 16: 99LN-BrM-associated myeloid cells contribute to an immuno-suppressive TME. a) Schematic overview. b) Normalized Counts of selected genes related to major myeloid lineages. Data are derived from comparing all controls vs. all TAMs, and are represented as bar graphs with individual values as dots. c) Principle component analysis (PCA) of control vs. BrM-associated myeloid cell types. $n=3$ for each condition. d) Euler Plots representing unique and overlapping DEGs for both, up (left) and down (right) regulated genes of the three major myeloid populations of interest, which were selected based on basemean $>20$ and padj. value $<0.05$. e) Heatmap of Top 50 DEGs within the MG or MDM compartment, comparing the TAMs with their respective normal cellular counterpart (control). All up- and downregulated genes within each group of comparisons are highly significant (padj $<0.001,{ }^{* * *}$ ). Certain gene names are indicated. f) Immuno-fluorescence staining of CD3 (red) and CD206 (green) on a 99LN-BrM thick brain section. DAPI (blue) was used as nuclear counterstain. Scale bar $=100 \mu \mathrm{m}$, inlay $=25 \mu \mathrm{m}$. g) Relative expression level (vst values) of certain indicated myeloid marker, important for the interaction between the myeloid and lymphoid compartment. Data derived from single comparisons of control vs. TAM (blood monocytes vs. TAM-MDM or normal MG vs. TAM-MG). Significance based on padj. values with $p<0.05\left(^{*}\right), p<0.01\left(^{* *}\right)$, and $p<0.001\left(^{(* *}\right) . N=3$ for each condition. Data deposited at GSE164049 and published in Niesel et al., 2021. 
3. The myeloid-rich H2030-BrM TME allows exploration of myeloid cell states under different conditions

The cellular and molecular characterization of TAMs and TILs in the TME of 99LNBrM further strengthened the importance of each. Since especially both major TAM populations are supposed to critically contribute to BrM progression, the following chapters will focus on the myeloid compartment. Therefore its alterations upon tumor induction and during BrM progression were examined within the myeloid-rich H2030BrM TME (figure 17) (Schulz et al., 2020).

RNA-Seq data of treatment-naïve BrM-associated myeloid cells were derived from BLI-based, macro-dissected BrMs of small or large stage tumors (figure 17a,b,c). Here, in total 3 - 5 samples of each cell type (TAM-MG, TAM-MDM, infl. monocytes (TA-Mono), granulocytes) from both, small (mean BLI value $4 \times 10^{7}$ ) and large (mean BLI 2.3 $\times 10^{8}$ ) BrMs were obtained (figure 17c). In accordance to results of the 99LNBrM model (Figure 16), PCA of all control vs. BrM-associated cell types revealed the highest variance coming from the cell type (PC1) and the cellular condition (PC2, control vs. BrM) (figure 17d). Moreover, TAM-MDMs and TA-Monos showed higher similarity of gene signatures compared to TAM-MG or granulocytes as evidenced by closer clustering (figure 17d,e). Since all BrM samples from every cell type clustered tightly, it was tempting to speculate that tumor progression lacked any influence on the transcriptome of myeloid cells. Hence, direct comparisons of small vs. large stage BrM-derived samples of TAMs and granulocytes (TA-Granu) (figure 17e) were performed. This analysis revealed the lack of differences between the transcriptomes of TAM-MGs, TAM-MDMs, TA-Monos, and TA-Granus during BrM progression (small vs. large) (figure 17e,f). Comparing the number of DEGs between different conditions clearly showed that BrM induction (control vs. small BrM-derived samples) most strongly affected the transcriptional signatures in TAM-MG (1119 DEGs), TAMMDMs (6225 DEGs), and TA-Monos (6596 DEGs). In contrast, BrM progression (small vs. large) resulted in only a few DEGs in every cell type (TAM-MG: 2; TAMMDM: 7; TA-Mono: 8), with most of them (21) belonging to the TA-Granu population (figure 17f). 
a

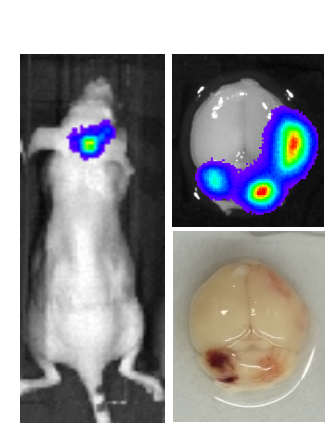

b

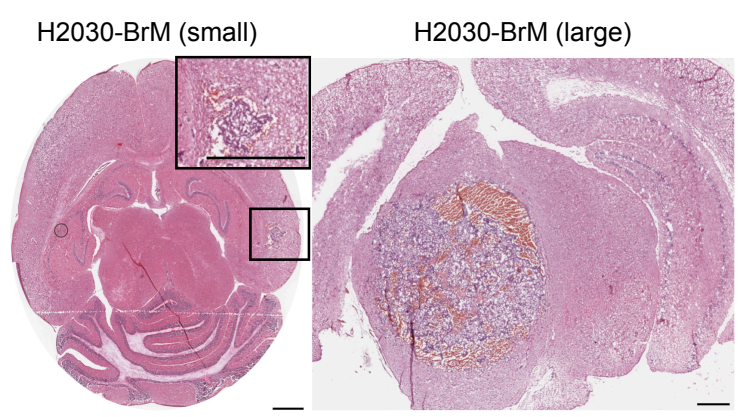

C

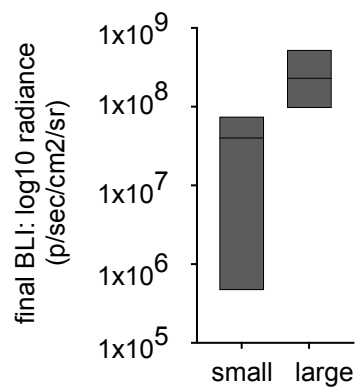

d

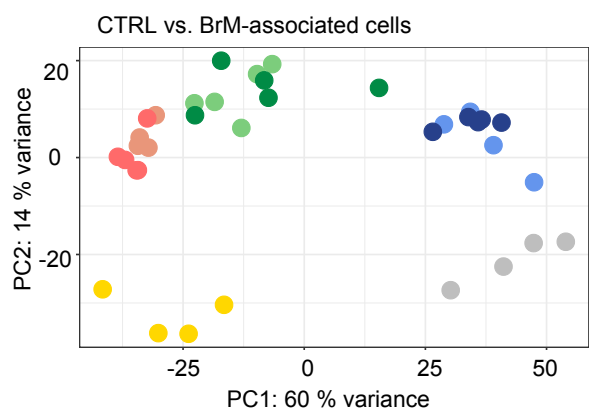

e
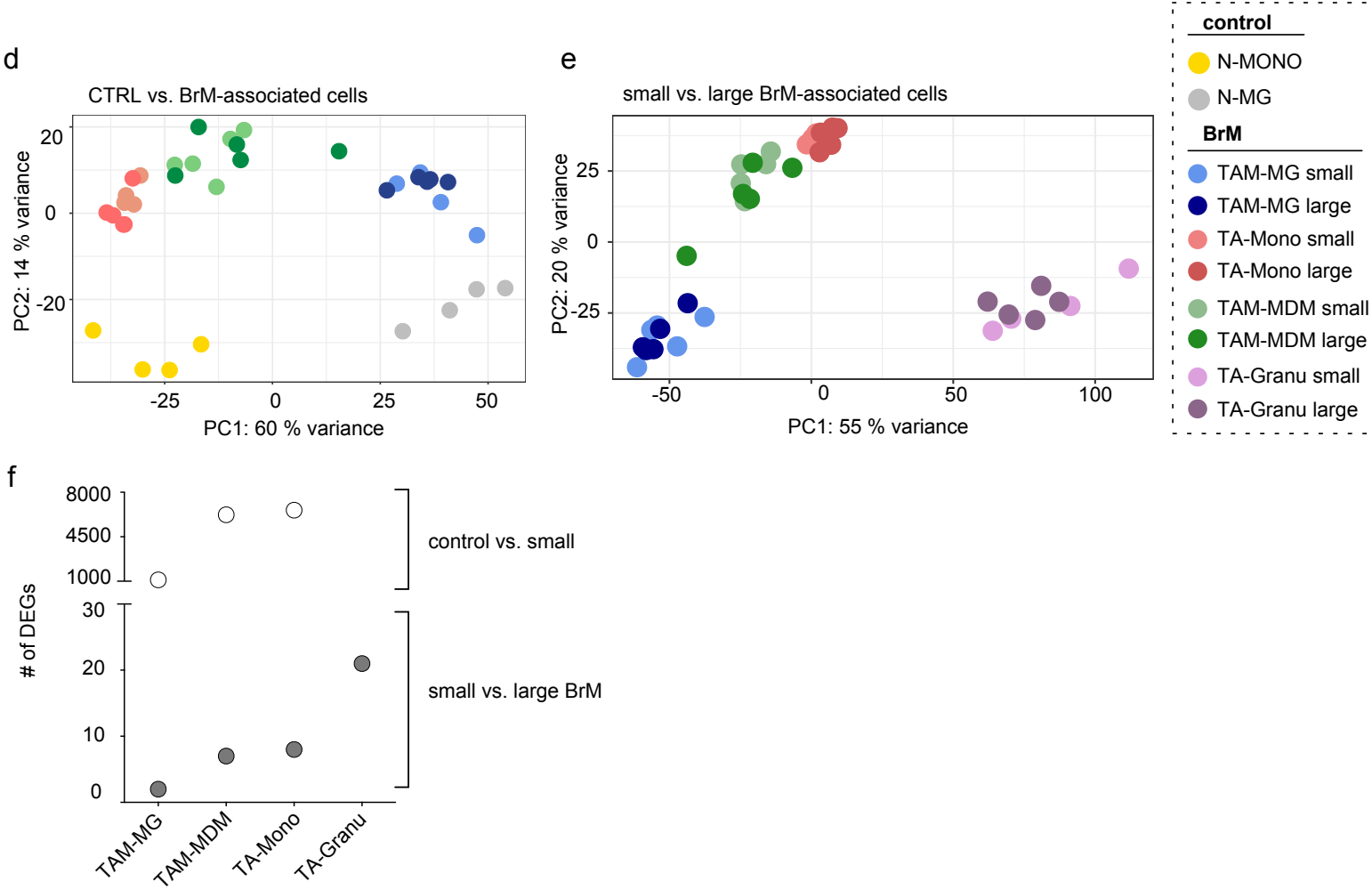

Figure 17: The $\mathrm{H} 2030$ lung cancer BrM model. a) Macroscopic images of the BLI signal of a representative $\mathrm{H} 2030-\mathrm{BrM}$ bearing mouse and a photograph of the corresponding brain. b) H\&E stainings of brain sections with small (left) or large (right) H2030-BrMs. Scale bar depicts $1 \mathrm{~mm}$. c) Final BLI values of treatment-naïve H2030-BrM bearing mice used for RNA-Seq. $n=5$ for each condition. d) PCA plot of control vs. all BrM-associated cells ( 3 TAM populations from two different stages). $\mathrm{N}$ = 3 - 5 per condition, as indicated in the color legend. e) PCA plot of small vs. large stage BrM-associated cell types, including TAM-MG, TAM-MDMs, TA-Monos, and TA-Granu. N = 3 - 5 per condition, as indicated in the color legend. f) Graph depicting the number of differently expressed genes (DEGs) within the indicated comparisons in the 4 major myeloid cell types from H2030-BrM. Data based on BM > 20 and padj. value $<0.05$. Note that all data have been published before, and plots are partially modified from Schulz et al., 2020.

In summary the data revealed that myeloid immune cell recruitment upon BrM induction most strongly affected the transcriptomes of TAM-MGs, TAM-MDMs, and TA-Monos in H2030-BrM. Contrary to that, transcriptomes examined during different stages of BrM were very similar, suggesting that tumor progression had no influence on molecular signatures, at least in H2030-BrM (Schulz et al., 2020). 
4. WBRT slightly changes the transcriptomes of different BrM-associated myeloid cell populations

Histology and FCM experiments showed that WBRT has the potential to affect the recruitment and infiltration of different lymphoid and myeloid immune cells (figures 11 - 14). To further investigate the molecular effects of WBRT on TAM transcriptomes, fractionated and hypo-fractionated WBRT was performed on H2030-BrM-bearing mice. Consecutively samples were sorted, and analyzed via RNA-Seq at different time points following IR (figure 18a) (Schulz et al., 2020). The comparison of all treatment-naïve vs. all treated samples revealed no changes in cellular identity upon application of treatment for each of the four cell populations. Treatment-naïve and treated samples of the different cell populations clustered close together in a PCA plot and did not reveal any distinction based on condition (figure 18b). To further evaluate the effects of WBRT on the population-wide transcriptomic signatures, systematic comparisons of untreated, large stage BrM samples vs. treated samples of every timepoint were performed within each cell type (figure 18c). As indicated, the numbers of DEGs for every comparison was relatively low (below 150) compared to the control vs. BrM situation as shown before (figure 17f). However, TAM-MG, TAMonos and TA-Granus showed highest amounts of DEGs in response to $1 \times 10$ Gy compared to $5 \times 2$ Gy at d3. Contrary, this effect was inversed in TAM-MDMs, which in every comparison revealed a number of DEGs below 100 (figure 18c). Together, this data point towards a high heterogeneity in distinct cell populations upon treatment, and that WBRT lacked strong effects on the molecular signatures of the myeloid bulk cell populations. This fact is illustrated in the comparison of RNA-Seq data comprising control microglia vs. TAM-MG at d3 upon $5 \times 2$ Gy. Within this comparison control, irradiated microglia as well as TAM-MG at d3 upon 1 x 10 Gy were included. An unsupervised clustering heatmap of these microglia clearly showed that the presence of BrM itself was the major driver of transcriptomic changes, at least among the Top 100 DEGs in TAM-MGs (figure 18d). Regardless of the WBRT scheme applied, TAM-MG upregulated the pro-inflammatory marker Tnf, the complement member $\mathrm{C}$, or genes related to ECM organization and cell-cell interaction ( $A x I, C D 72$, Ctsb, Mmp12). In contrast, normal control microglia, both with and without WBRT, showed higher gene expression of the myelin basic protein (Mbp), the connexin Cx43, or Tspan15, which as a protein is part of the metalloprotease ADAM10 (Koo et al., 2020). Interestingly, Sema4b was 
downregulated in response to $\mathrm{BrM}$ as well. The protein encoded by this gene is involved in maintenance of synapse integrity (Pasterkamp and Giger, 2009). To finally understand the effects of different WBRT schemata on the TME in BrM, and its different immuno-modulatory modes of action, functional annotation and pathway analyses were performed (Schulz et al., 2020). The combined DEGs from all H2030TAMs isolated at $d 3$ upon $1 \times 10$ Gy or $5 \times 2$ Gy were subjected to these analyses (figure 18e). While cells from both settings upregulated genes related to stress response pathways, the single application of $10 \mathrm{~Gy}$ induced further pathways related to host defense mechanism in the overall TME (figure 18e). Moreover the Top 5 annotations and pathways resulting from downregulated genes were different between both experimental settings, as well. While the classical fractionated WBRT scheme resulted in downregulation of genes related to brain homeostasis (e.g. "regulation of neuron projection development", "dendrite development"), WBRT applied as single high doses interfered with "leukocyte migration", and the "regulation of responses to external stimuli" (figure 18e). Although the TAM-MDM compartment was more efficiently depleted at $\mathrm{d} 3$ after $1 \times 10 \mathrm{~Gy}$ in H2030-BrM (figure 11d), the rapid re-infiltration and continuous influx together with RNA-Seq data suggests that distinct cell types in the TME show higher population heterogeneity upon irradiation. This effect most likely resulted from treatment-naïve and newly recruited cells, which together form the "post-IR" TME. 
a

Treatment naive vs. irradiated TME

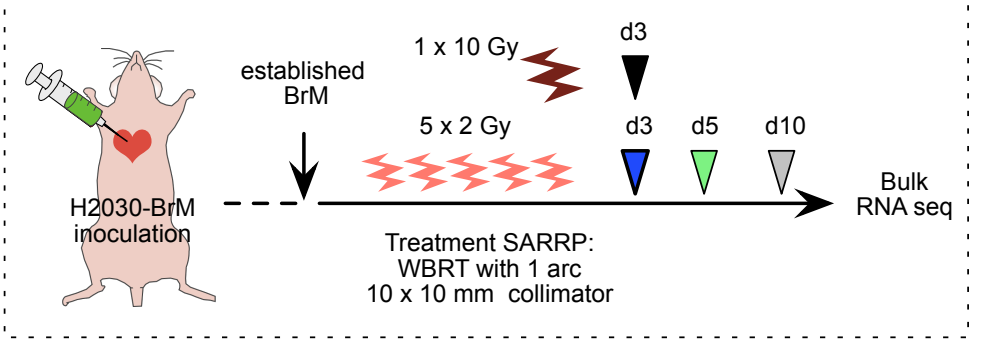

b

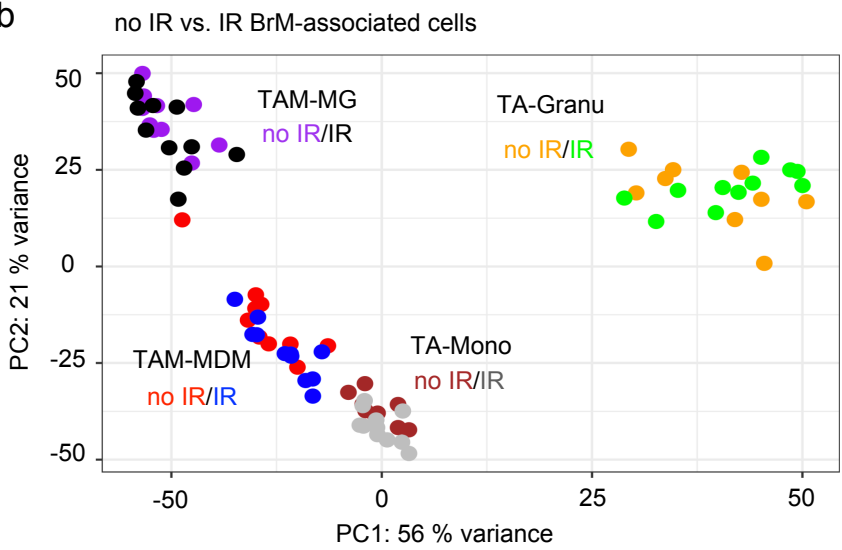

d

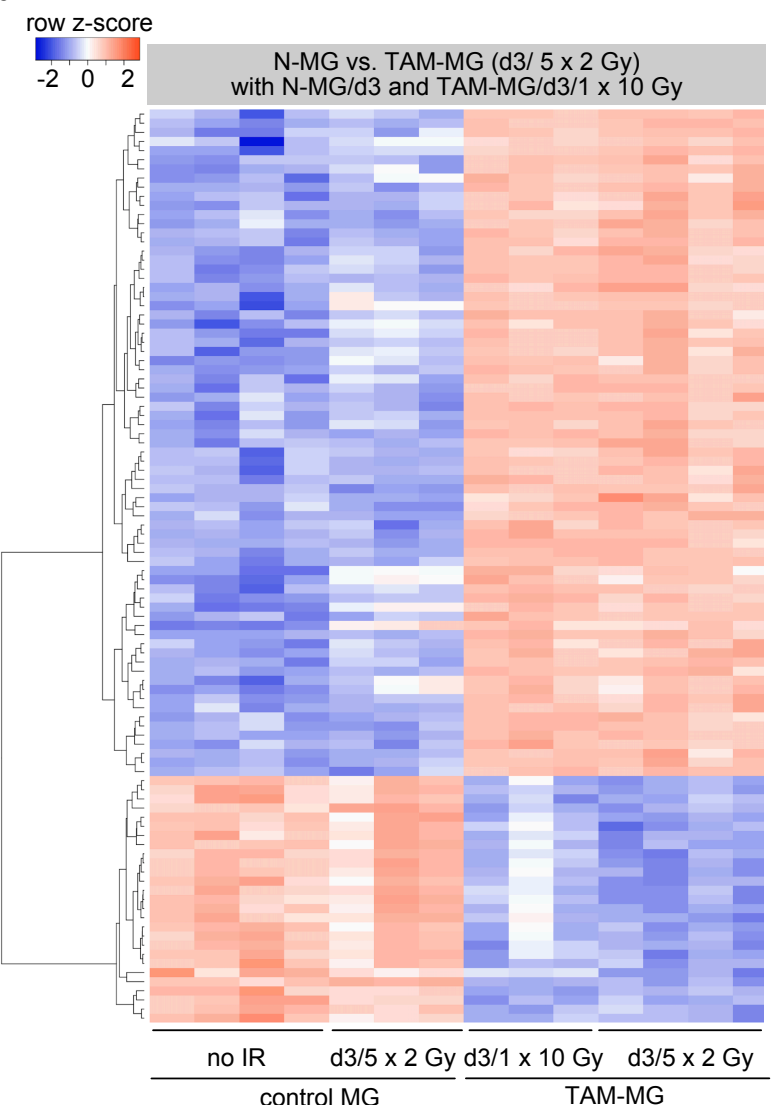

days after IR

C

d3 (5 x 2 Gy) d3 (1 x 10 Gy):

$\therefore$ d5 (5 x 2 Gy)

$\checkmark$ d10 (5 x 2 Gy)

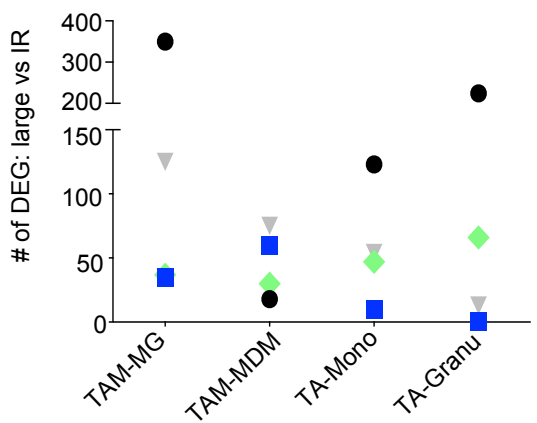

e

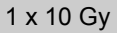

$5 \times 2 \mathrm{~Gy}$

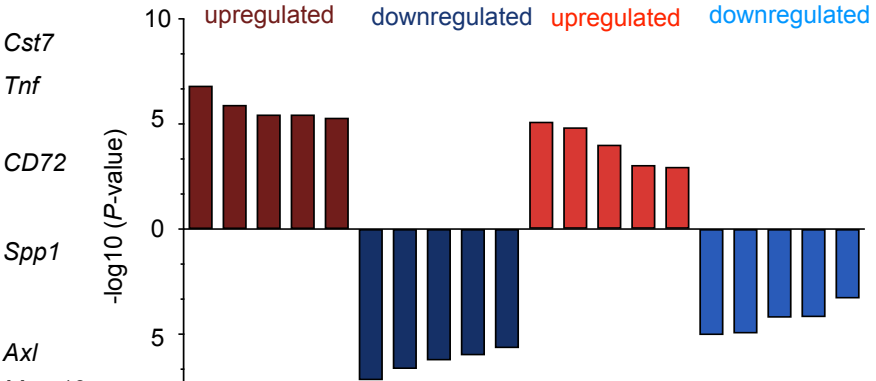

Mmp12

Ctsb

C4b

C3

Mbp

Cx43

Tspan15

Sema4b

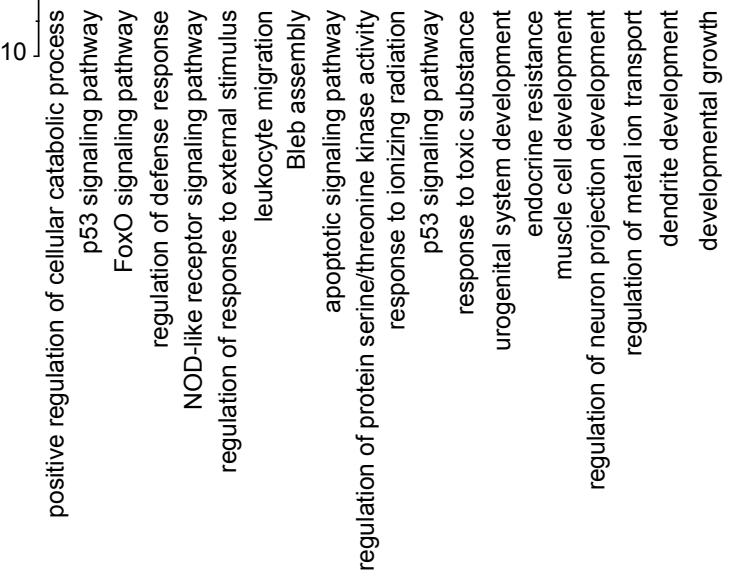


Figure 18: Molecular consequences of WBRT-treated H2030-BrM. a) Methodological overview. b) PCA plot of all $\mathrm{H} 2030-\mathrm{BrM}$ associated myeloid cells, comparing all no IR vs. all IR treated samples without further stratification. Each dot represents one bulk sample. c) Number of DEGs (cutoff: BM > 20, padj. value < 0.05 ) in different cell types by comparing RNA-seq data of large stage BrM-associated samples $(n=5)$ with its irradiated BrM-associated cellular counterparts at different days upon WBRT (5 x 2 Gy/d3 and d5 n = 4, $1 \times 10 \mathrm{~Gy} / \mathrm{d} 3$ and $5 \times 2 \mathrm{~Gy} / \mathrm{d} 10 \mathrm{n}=3)$. d) Unsupervised hierarchical clustering heatmap depicting the Top 100 DEGs in N-MG vs. TAM-MG including irradiated N-MG at d3 upon $5 \times 2$ Gy, as well as TAM-MG at d3 upon $1 \times 10$ Gy WBRT, cutoff: BM $>20$, padj. value $<0.05$, generated with heatmapper. e) Top 5 gene annotation of all DEGs from each cell type combined in the indicated conditions (cutoff: BM $>20$, adj. $p$ value $<0.05$; log2 fold change). Note that all data have been published before, and are partially modified from Schulz et al., 2020.

As previous analyses revealed that the TAM pool consisted of several distinct cell populations based on their different developmental origins, it now was interesting to understand specific treatment-induced molecular gene expression pattern in irradiated TAMs. Hence, a set of comparative population-wide analyses was performed with TAM samples derived from non-treated and treated H2030-BrM (figure 19a). Comparing all TAM-MG samples vs. all TAM-MDM samples resulted in clear segregation of cell populations in PCA plots under treatment-naïve (figure 19b), and treated (figure 19c) conditions and additionally revealed a slight wider dispersal of TAM-MDM samples within both different contexts. This further suggests a greater heterogeneity within the MDM pool under various conditions. Given the fact that both TAM populations critically contribute to BrM biology and macrophages in tumors are associated to therapy resistance (Ruffell and Coussens, 2015), specific treatmentinduced transcriptional programs within each population were examined. Therefore, DEGs upregulated in either TAM-MGs or TAM-MDMs derived from both comparisons (no IR and IR) were identified (figure 19d). This comparison of DEGs impressively demonstrated that although in both cell types the majority of TAM-specific DEGs is similar under treatment-naïve and treated conditions, there is a substantial set of DEGs unique to either or condition (figure 19d). The core TAM set included approximately 1.900 and 2.100 DEGs in TAM-MG, and TAM-MDM, respectively. The treatment-specific (IR) set of DEGs comprised about 1.500 DEGs in TAM-MG (figure 19d left), and about 1.000 DEGs in TAM-MDM (figure 19d right). 
a

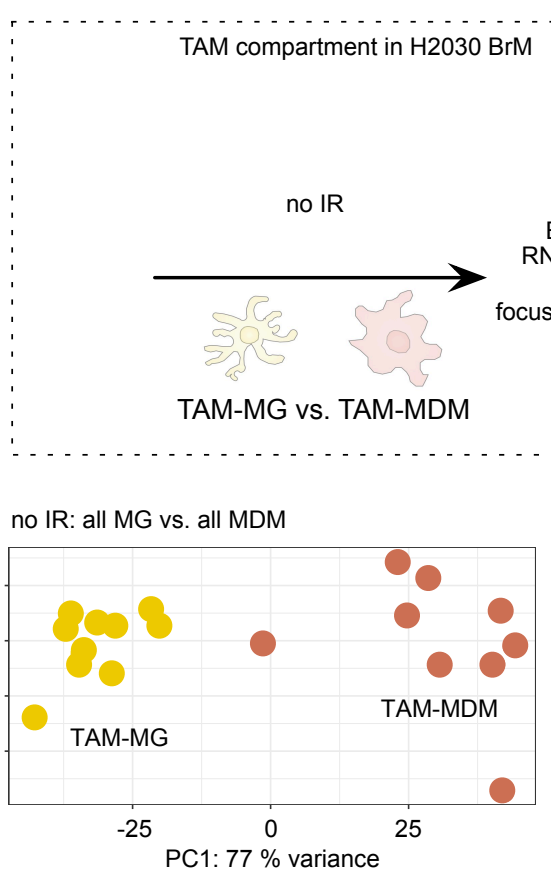

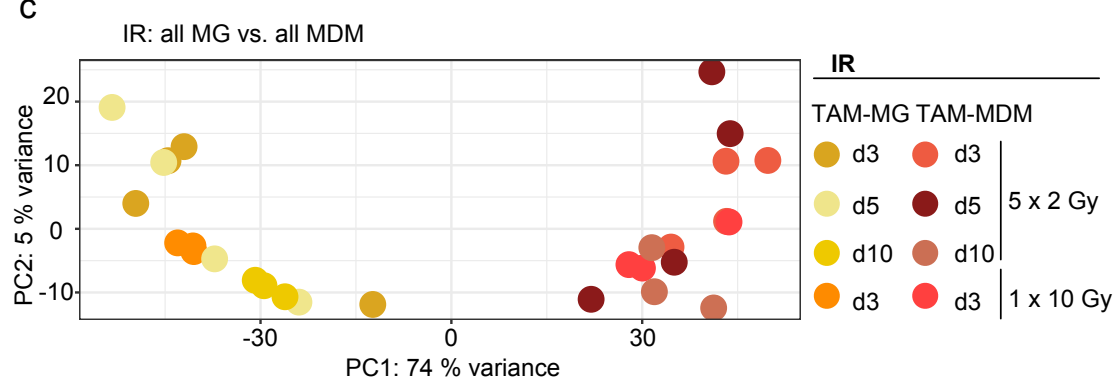

d

e

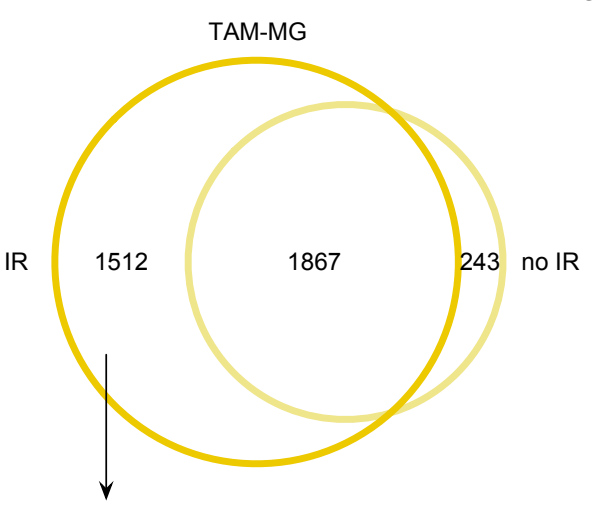

TAM-MG vs. TAM-MDM

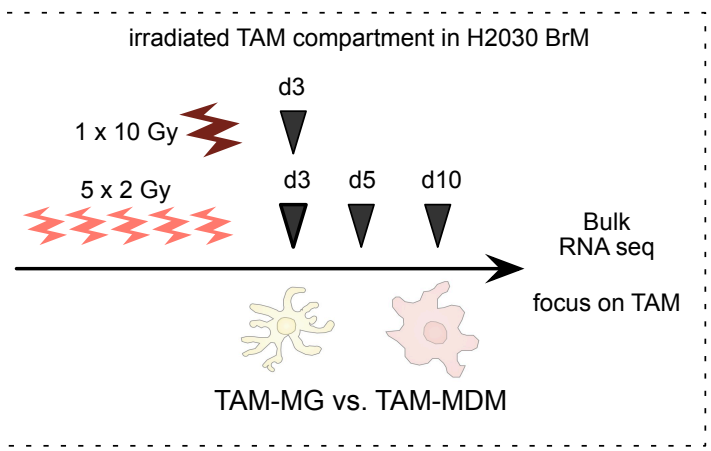

b

C

vs.

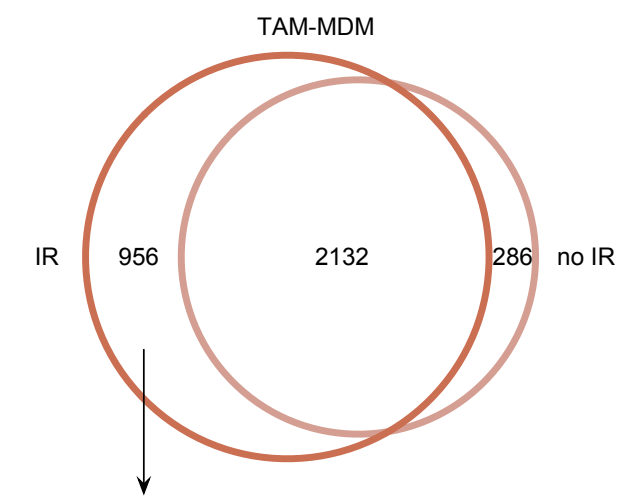

TRRUST analysis

(Transcriptional Regulatory Relationships Unraveled by Sentence-based Text mining)

\begin{tabular}{|c|c|c|c|c|c|}
\hline \multirow{2}{*}{ regulated by: } & \multicolumn{3}{|c|}{$-\log (p)$} & & \\
\hline & 0 & 24 & 6 & & \\
\hline $\operatorname{Trp53}$ & & & Atf3 & Cdkn1a & Dusp1 Fos Pias3 \\
\hline Hdac2 & & & Ccne1 & Cdkn1a & Nfkbia Smarca4 \\
\hline Snai2 & & & $B b c 3$ & Fzr1 & Nckap1 Ocln \\
\hline Tfdp1 & & & Cdkn1a & Myb/2 & $T c f 3$ \\
\hline Twist2 & & & Add1 & Cdkn1a & Srebf1 \\
\hline
\end{tabular}

\begin{tabular}{|c|c|c|c|c|c|}
\hline regulated by: & $-\log (p$ & & & & \\
\hline 0 & 2 & 4 & & & \\
\hline Jun & & Ctsk & $\operatorname{ltg} a 5$ & Mmp9 & Msr1 Tnfrsf1b \\
\hline Crebbp & & $B c / 2$ & Fth1 & Ldha & Nfyb \\
\hline IIf4 & & Prdm1 & $C x c / 9$ & Spi1 & \\
\hline Parp1 & & $F n 1$ & Mmp9 & Vcam1 & \\
\hline Creb1 & & $B c / 2$ & Noct & Esr1 & Ldha Prkce \\
\hline
\end{tabular}


Figure 19: Transcriptional changes of irradiated TAMs in H2030-BrM. a) Schematic overview. b) PCA plot of all TAMs from non-irradiated H2030-BrM. All TAM-MG vs. all TAM-MDM were compared. $\mathrm{N}=9$ (no IR), and 19 (IR). c) PCA Plot of all irradiated TAMs from H2030-BrM, derived by comparing all IR TAM-MG vs. all IR TAM-MDM including all IR conditions as indicated. For both cell types: $n=3$ (d10 (5 x 2 Gy), d3 $(1 \times 10 \mathrm{~Gy})), \mathrm{n}=4$ (d3 and d5 (5 x 2 Gy)). d) Euler plot indicating unique and overlapping DEGs specifically upregulated in TAM-MG (left) or TAM-MDM (right) compared to TAM-MDM or TAM-MG, respectively from both, no-IR and IR comparisons. e) TRRUST analysis was performed via Metascape of all IR-specific DEG sets from TAM-MG (left), or TAM-MDM (right). Note that data in this figure are associated to GSE137797 (Schulz et al., 2020).

Transcriptional regulatory relationship analysis (TRRUST) of these DEGs finally allowed the identification of treatment-induced regulatory programs within both TAM populations (figure 19e). Not surprisingly, TAM-MG showed an enrichment of genes related to response to radiation, mitosis, and DNA replication/repair mechanisms. Hence, the Top 5 networks were dominated by genes regulated by Trp53, Hdac2, Snai2, Tfdp1, and Twist2, all of them well described in the above-mentioned processes (figure 19e left). The TAM-MDM pool showed treatment-specific genes higher regulated compared to TAM-MGs, which belong to RNA metabolism, innate immune responses and cytokine signaling, all of them in networks regulated by Jun, Crebbp, Irf4, Parp1, and Creb1 (figure 19e right).

Collectively these results suggest that both major TAM populations not only show distinct molecular signatures based on their ontological origin, but also that these transcriptional networks can be influenced by WBRT in a cell type-specific manner. This is of particular importance for the development of targeted therapies as adjuvants to radiotherapy. 


\section{Single cell RNA-Sequencing reveals TAM heterogeneity before and after WBRT}

The data of FCM experiments in treatment-naïve vs. treated H2030-BrM suggested that WBRT enhances the infiltration of TAM-MDMs. Consequently, the TAM pool was constantly replenished with newly recruited cells form the periphery. Hence, it was hypothesized that the total TAM-MDM population might display a higher heterogeneity compared to the TAM-MG population. In order to prove this, single cell RNA-Seq of TAMs was performed (figures 5, 20) (Schulz et al., 2020). Derived from two different experimental settings (treatment-naïve and WBRT-treated), sequencing yielded an average gene number of 1558 in 170 untreated, and 1125 in 190 irradiated TAM-MG. The average gene number in TAM-MDM was slightly higher (1707 untreated, 2622 treated), and was derived from 237 untreated, or 269 irradiated TAM-MDM (figure 20a). Together, all single TAMs contributed to a total of 16 different cluster, which were determined by tSNE analysis including cells with an UMI > 800 (unique molecular identifier) (figure 20b, c, d). Most importantly was the number of cells contributing to each of the cluster across all conditions (figure 20c). Defining one cluster as group of at least 20 cells, the majority of TAM-MG from both experimental settings contributed to three cluster each, i.e. cluster 4, 7, 9 in nontreated samples, and cluster 10,11, and 14 in irradiated ones. TAM-MDM however gave rise to four and six cluster, in untreated (cluster 1, 6, 8, and 10) or treated samples (cluster 1, 2, 10,12, 14, and 15), respectively (figure 20c, d). Furthermore, both non-treated cell types only minimally overlapped across the cluster, whereas irradiated samples were found to contribute to more overlapping cluster (cluster 10,14, and 15) (figure 20c, d), suggestive of some shared radio responses. These data indicate that TAM-MG represent a more homogenous population compared to TAM-MDM, and direct comparison further revealed molecular differences between both cell types (figure 20e) (Schulz et al., 2020). Whereas pro-inflammatory genes (e.g. $\mathrm{Cc} / 3$ ) and other regulatory MG genes (e.g. Fth1, Hexb, Sparc) were predominantly upregulated in TAM-MGs, several genes related to antigen processing and presentation (e.g. CD74, H2-Aa, H2-Ab1, H2-Eb1) were enriched across cluster consisting mainly of TAM-MDMs (figure 20e). In addition to the bulk RNA-Seq data, also scRNA-Seq data pointed towards molecular dichotomy of both TAM populations. Furthermore, TAM-MDMs represent a more heterogeneous population both, with and without WBRT. 
VI Results

a

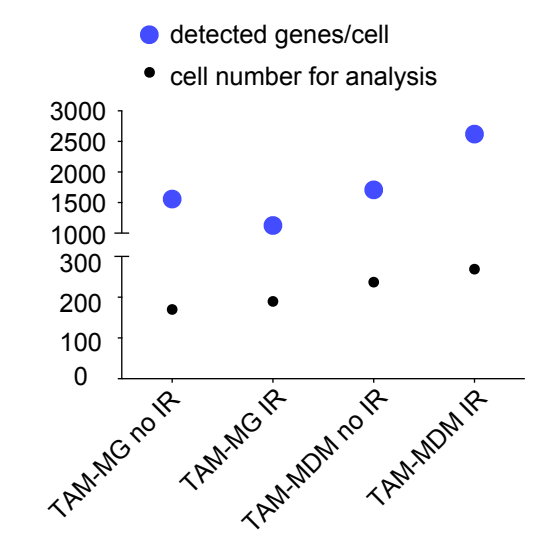

c

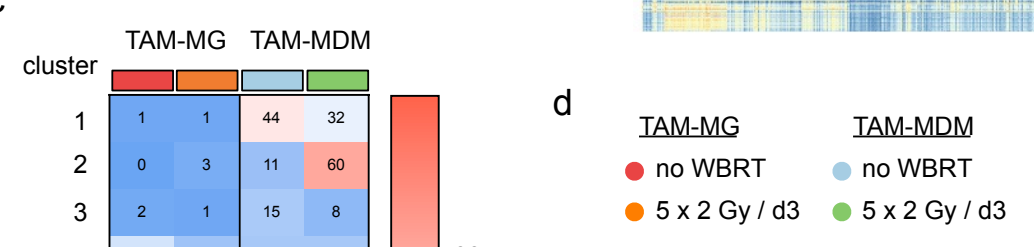

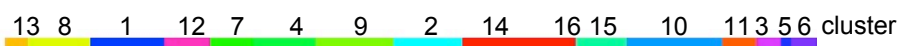
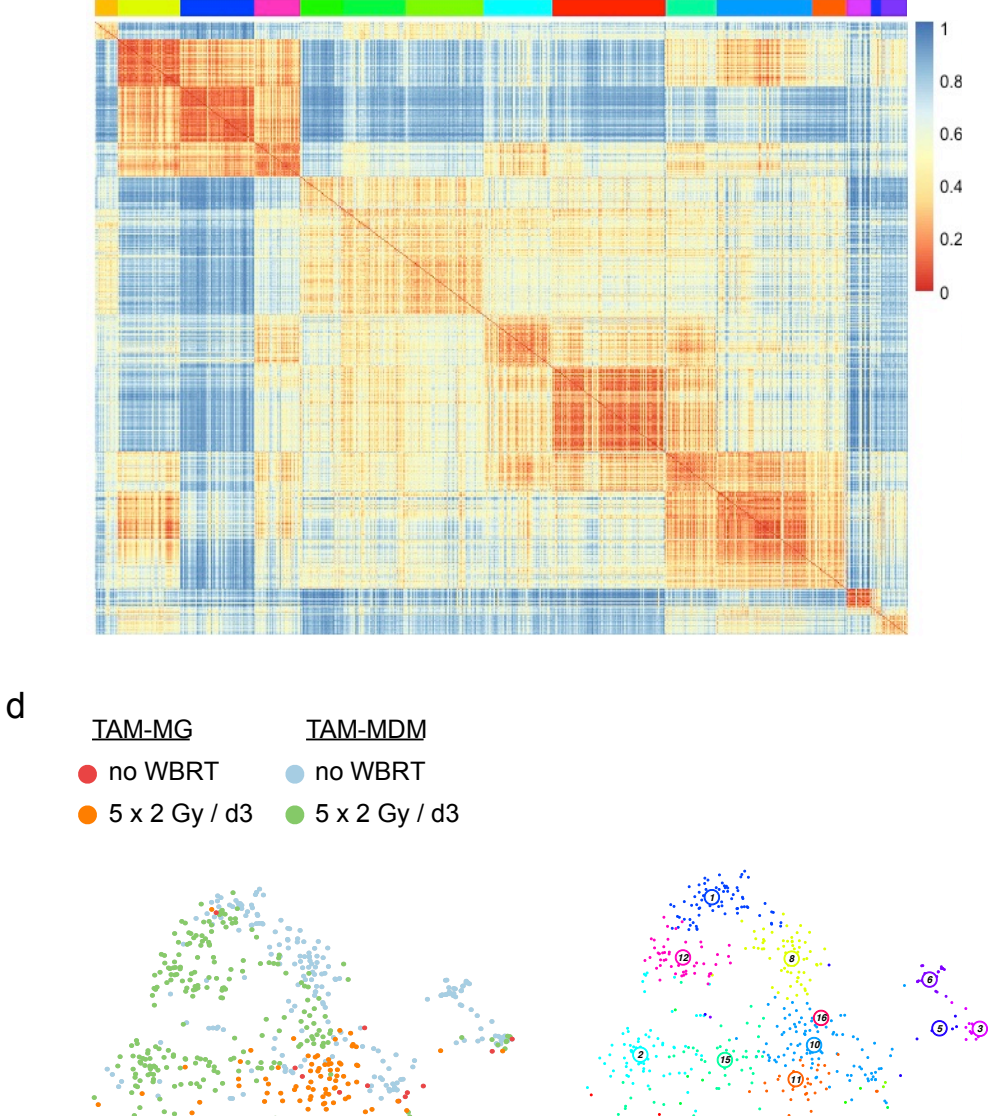

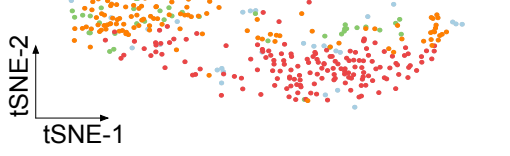
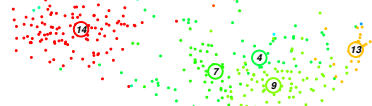

e
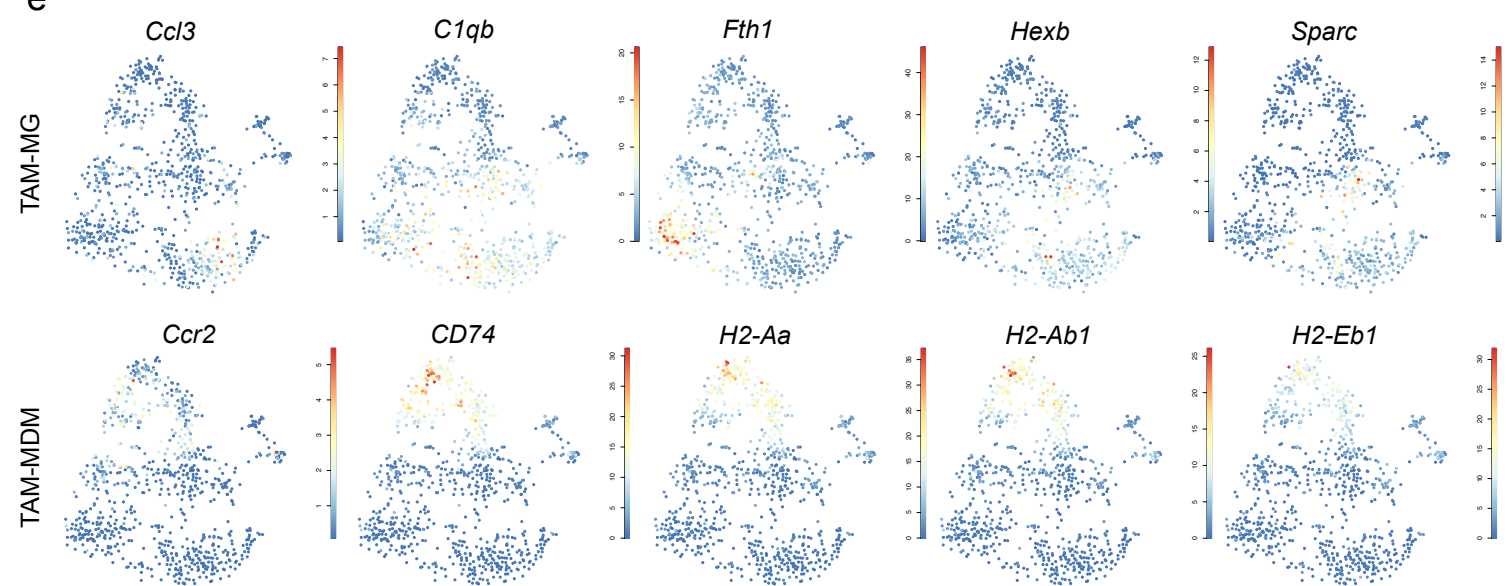
Figure 20: Single cell RNA-Seq of TAMs in H2030-BrM with and without WBRT. a) Average number of detected genes per cell and the cell number used for analysis across all experimental groups. b) Correlation plot depicting clustering of the analyzed cells of each experimental condition. c) Heatmap depicting the number of cells per condition contributing to the individual cluster. d) tSNE plots representing all individual cells from every condition of treatment-naïve and treated TAMs within their respective cluster (right). $\mathrm{N}=1 \mathrm{BrM}$ per condition, yielding a cell number as indicated in a. e) Individual tSNE plots of representative genes belonging to either TAM-MGs (upper row) or TAM-MDM (lower row). Note that all data have been published before, and are partially modified from Schulz et al., 2020. 
6. Cross-model comparative analyses of RNA-Seq data reveals BrM-specific transcriptomic profiles and a core BrM gene set of TAM-MG and TAM-MDM

Analyzing transcriptomic profiles of immune cells associated to one model resulted in deep insight into molecular changes in inflammatory cells occurring in response to BrM. While every cell type showed distinct signatures, especially TAM-MGs and TAM-MDMs from H2030 and 99LN showed also a considerable overlap and similarly regulated genes within the Top regulated ones. Hence, in order to identify common genes regulated in TAMs across different models, a comprehensive cross-model comparison was performed (figures 21 - 25). Samples consisted of all controls and all TAMs from H2030 (Schulz et al., 2020), and 99LN BrM (Niesel et al., 2021). In addition, a data set from a xenograft breast-to-brain metastases model (MDA) was included (Klemm et al., in revision) in silico.

Subjecting all samples stratified as control vs. TAM into analyses revealed the existence of basically four groups of cell cluster regardless of the underlying model: blood monocytes, normal microglia, TAM-MG, and TAM-MDM (figure 21b), similarly as seen before. The strongest variances herein resulted from either the cell type (PC1) or the condition (PC2, control vs. BrM), regardless of the underlying model (figure 21b). In order to identify similarities across the different models, single comparisons were performed for control vs. TAMs of each model, as partially shown before. Comparing up- or downregulated genes across the models, and compiling similarly regulated genes within Euler Plots revealed a comprehensive set of unique and overlapping DEGs within and between each model (figure 21c,d). Very important to note was the overlapping set of genes between similar backgrounds (i.e. MDA and $\mathrm{H} 2030$, both Balb/c), and similar tumor entities (i.e. MDA and 99LN, both B2B but from a different background). Consequently there were approximately 100 genes similarly upregulated in TAM-MG from Balb/c nude mice, whereas about 60 were jointly downregulated. TAM-MDMs of that background showed 370 upregulated, and about 60 downregulated DEGs (figure 21c,d). Of note, the similarly regulated genes from both B2B metastasis models were slightly higher in both cell types (figure 21c,d). About 220 and 240 DEGs were similarly upregulated in TAM-MG or TAMMDM, respectively. On the other hand, there were 110, and 170 DEGs analogously downregulated in TAM-MG, and TAM-MDMs of both B2B models. But most interesting was the set of commonly regulated genes across all three models. In TAM-MG about 260 DEGs were uniformly up-, and about 90 genes commonly 
downregulated. The genes similarly upregulated included members of cytokine signaling, interleukin signaling, and inflammatory responses (e.g. Cc/2, Cc/5, Cc/7, Ccl8, Ccl12, Csf1, Cxc/1, Cxc/2, Cxc/13, Il1b, I/15ra, Il2rg, I/4ra), complement components (C2, C3, C4b, C5ar1), genes related to TNF (Tnf) and interferon signaling (e.g. Ifit2, Ifitm2, Ifitm3, Irf7, Zbp1), as well as members related to cell cycle and mitosis (e.g. Aurka, Aurkb, Bub1) (figure 21c, figure 22). Functional annotation further yielded related pathways, which were commonly upregulated including "migration", "inflammatory responses", "cell cycle", or "activation of complement members" (figure 22). In contrast, common downregulated genes included e.g. Adamts16, Bsn, Cask, Col26a1, Cux2, Gp9, Lrba, Nav2, the MG homeostatic marker Sall1, the semaphorins Sema4b, and Sema6d, or TIr5. Not surprising was the functional annotation of these DEGs, in which several terms and pathways related to brain homeostatic functions (e.g. "neurotransmitter release cycle", "neuron differentiation") could be revealed across TAM-MGs from all models (figure 22). In depth characterization of uniformly upregulated genes in TAM-MDM revealed a certain overlap to TAM-MGs but also a different set of regulated genes of in total approximately 280 DEGs upregulated, including complement members (e.g. C1qb, C1qc, C4b, C5ar1), members of cytokine and interleukin signaling $(\mathrm{Cc} / 3, \mathrm{Cc} / 4, \mathrm{Ccl} /$, Ccl8, Ccl12, Csf2rb2, Cxcl10, Cxcl13, Cxcl16, I11a, II1b, I/4ra), cathepsins (Ctsa, Ctsb, Ctsc, Ctsd, Ctsf, Ctss, Ctsz), members of angiogenesis and cell-cell interaction (e.g. Axl, Gas6, Vegfb), purinergic receptors (P2ry6, P2ry10, P2ry13), and most importantly, again genes related to interaction with the lymphocyte compartment (e.g. $C D 72, C D 74, C D 83, C D 86, H 2-A a, H 2-E b 1$ ) (figure 21d). Very interestingly, Tmem119, the homeostatic microglia specific marker was upregulated in TAM-MDMs across all models as well, indicating the molecular complexity to differentiate between both TAM populations (figure 21d). This was also shown for the human BrM scenario (Klemm et al., 2020). Functional annotation resulted in common upregulated pathways related to for example "lysosome", "inflammatory responses", or "ECM organization" (figure 22). Among the 165 commonly downregulated genes were e.g. Ace, Bc/2, CD44, F5, Foxp1, II17ra, II31ra, the myelin basic protein Mbp, Notch2, Runx2, the cell adhesion molecule Spn, Tgfbr3, or Trem3. Annotated downregulated pathways of these genes were related to e.g. "homeostasis", "extravasation", and "cell differentiation" (figure 22), further suggesting profound molecular adaptation of peripheral cells upon entry into the brain. 
Together, this comparative analysis uncovered the transcriptional dichotomy of both TAM populations within a multi-modal approach and further point towards their nonoverlapping function within the BrM TME.

a

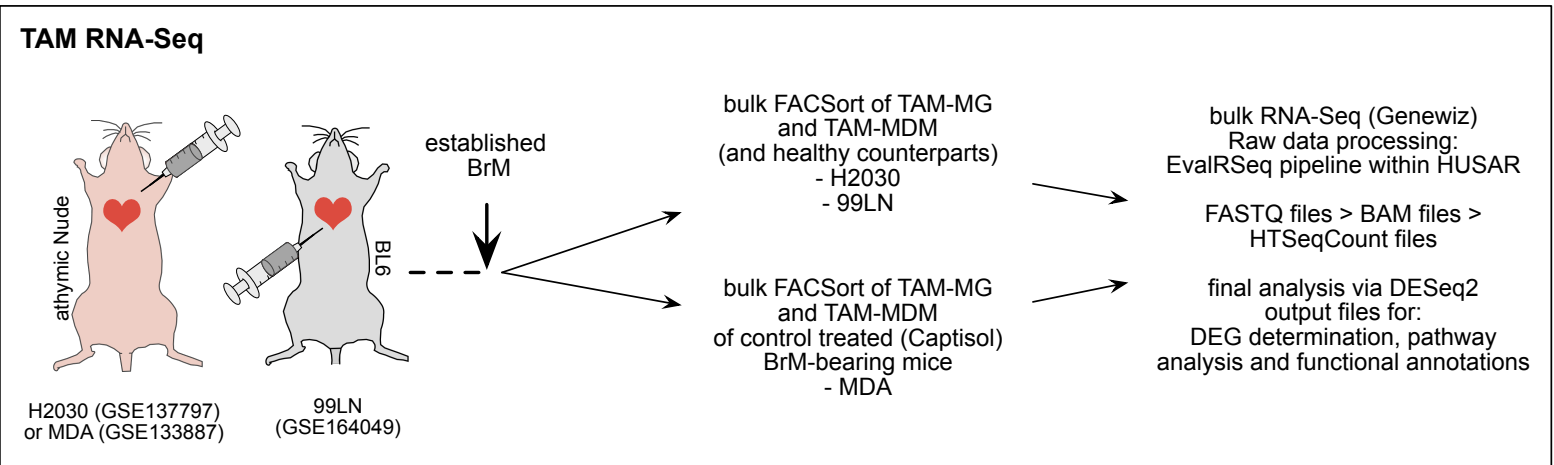

b

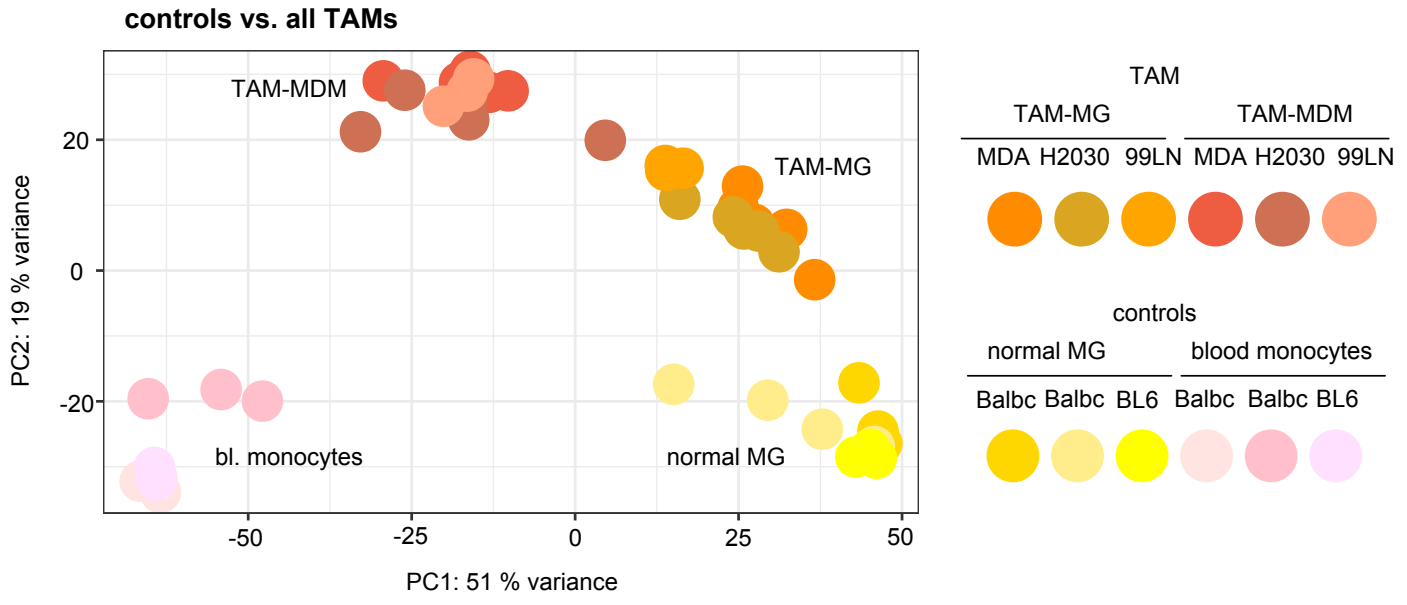

C

TAM-MG
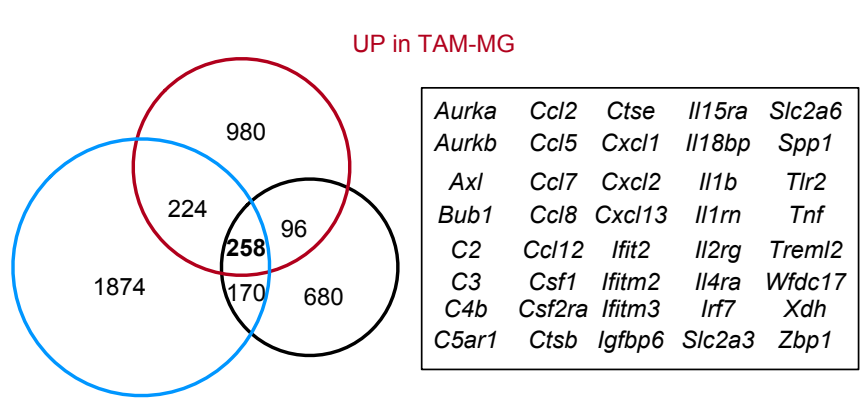

DOWN in TAM-MG

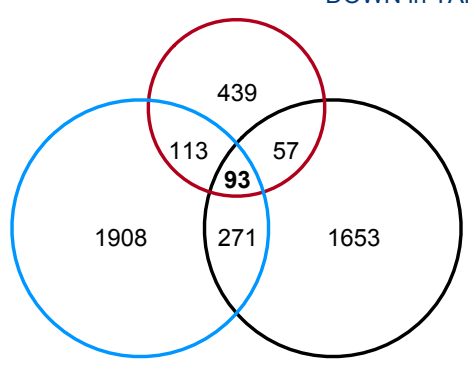

Adamts16 Dbp Nhs/2 Tjp1 Apba1 Fhit Ptn TIr5 Arvcf Gp9 Sall1 Tnfrsf17 Bank1 Hrh2 Sema4b Ttll3

Bsn Lrba Sema6d Zfp37 Cask Mtss1 Slc1a2 Zfp69 Col27a1 Myl2 Slc2a5 Zfp691 Cux2 Nav2 Smpd5 Zfp827 d

TAM-MDM
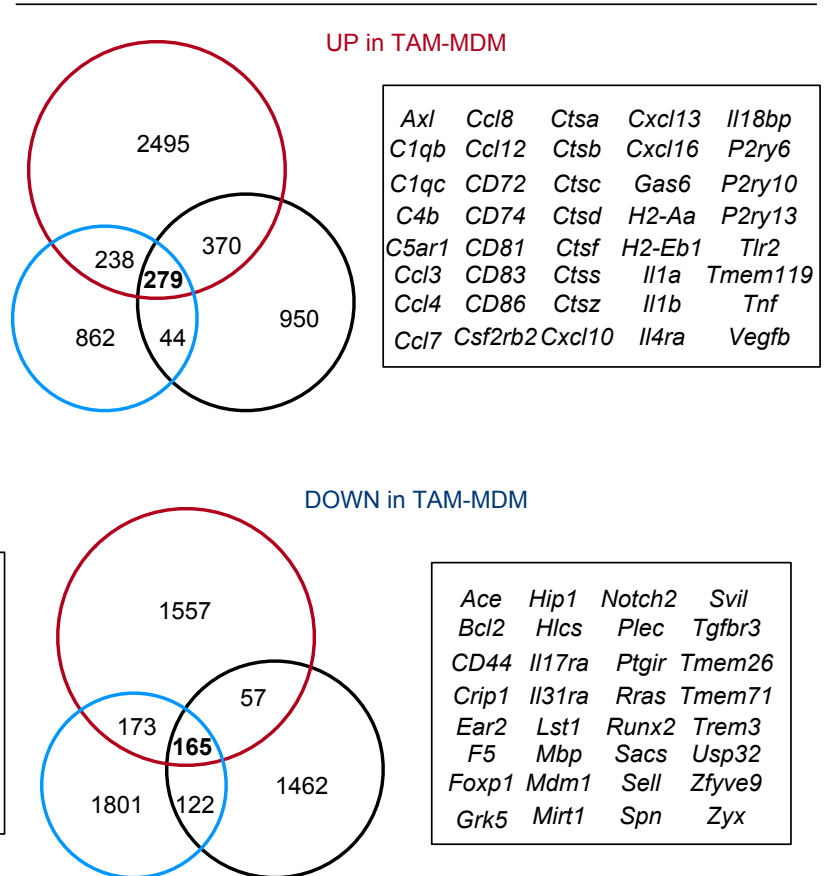
Figure 21: Multimodal comparison of TAM RNA-Seq data. a) Methodological overview. RNA-Seq data of TAM-MG and TAM-MDM of the following three different models were used for the figures 20 - 24: H2030 (GSE137797), MDA (GSE133887), and 99LN (GSE164049). b) Principle component analysis (PCA) of all controls vs. all TAMs from the three different models. $\mathrm{N}=3-5$ per condition and cell type. c) Euler Plots representing the number of DEGs per individual comparison from both TAM cell types within each model. Data derived from standard cutoffs: BM > 20 and padj. value $<0.05$. Up- or down-regulated was defined based on positive (up) or negative (down) log2 fold changes. d) Overlapping commonly upregulated example genes from TAM-MGs (left), and TAM-MDMs (right). Note that data are partially published and were derived from H2030-BrM (Schulz et al., 2020), MDA-BrM (Klemm et al., in revision), and 99LN (Niesel et al., 2021).

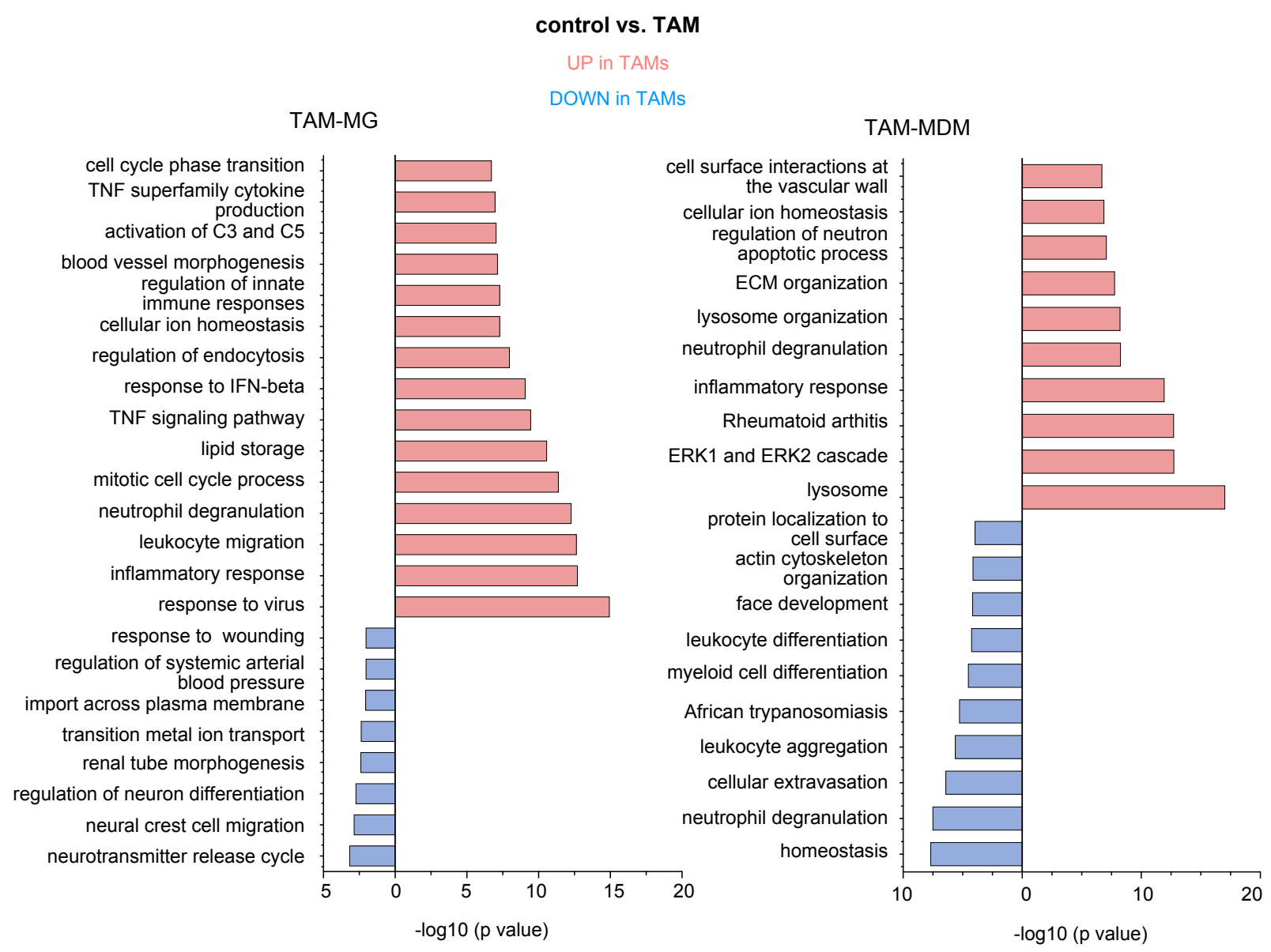

Figure 22: Functional annotation of commonly regulated genes in TAMs. Functional annotation and pathway analyses of commonly up- or downregulated genes in TAMMG and TAM-MDMs. Overlapping common genes were subjected to Metascape analyses and results plotted via Prism. Note that data are partially published and were derived from H2030-BrM (Schulz et al., 2020), MDA-BrM (Klemm et al., in revision), and 99LN (Niesel et al., 2021). 


\section{TAM-MG and TAM-MDM represent two distinct cell types in BrM}

While the comparison of TAMs with their respective normal cellular counterparts impressively demonstrated the differences in their transcriptomes upon BrM education, a direct comparison between both cell types was performed in order to clearly delineate each other's putative functions (figure 23). Grouping all TAM-MG derived from H2030, MDA, and 99LN, and comparing them with all TAM-MDM revealed a total of 1197 genes upregulated in TAM-MG, while the number in TAMMDM was slightly higher, i.e. 1465 (figure 23a). Interestingly among the Top 100 genes, the majority was upregulated in TAM-MDMs (figure 23b). Among the few DEGs higher expressed in TAM-MG was Adgrg1. The other Top regulated genes higher expressed in TAM-MDM contained the C-type lectin-like receptor Clec9a, which is implicated to be responsible in cytokine production (Huysamen et al., 2008). In addition, genes related to cell-cell, and cell-environment interaction (Adam19, Itga4, Mmp13, P2ry10, Shtn1), the aryl hydrocarbon receptor Ahr, the interferon induced protein Ifitm1, and the complement regulator Cfp were much higher expressed in TAM-MDM (figure 23b).

Using all identified genes as input for functional annotation resulted in clearly distinct putative functions of both TAM populations within the TME of different BrM models. In this context the involvement of genes within TAM-MG developing inflammatory phenotypes, including "lipid and glycoprotein processes", "cell-cell adhesion", "cell migration", and "cell morphogenesis" was very striking (figure 23c). Notable, the term "Arachidonate production from DAG" deserves attention, since arachidonate is a fatty acid metabolite implicated in neurodegeneration and -toxicity (Brown and Neher, 2010). On the other side, TAM-MDMs commonly upregulated genes related to "migration", "induction of inflammatory and defense responses", "regulation of cytokine production", and "responses towards interferon gamma". Most importantly, in this gene list several terms appeared which were associated to interaction with the lymphocyte compartment ("T cell activation", and "positive regulation of immune responses"), again arguing for the importance of this TAM population for interacting with the adaptive immune system (figure 23c). 
TAM-MG vs. TAM-MDM
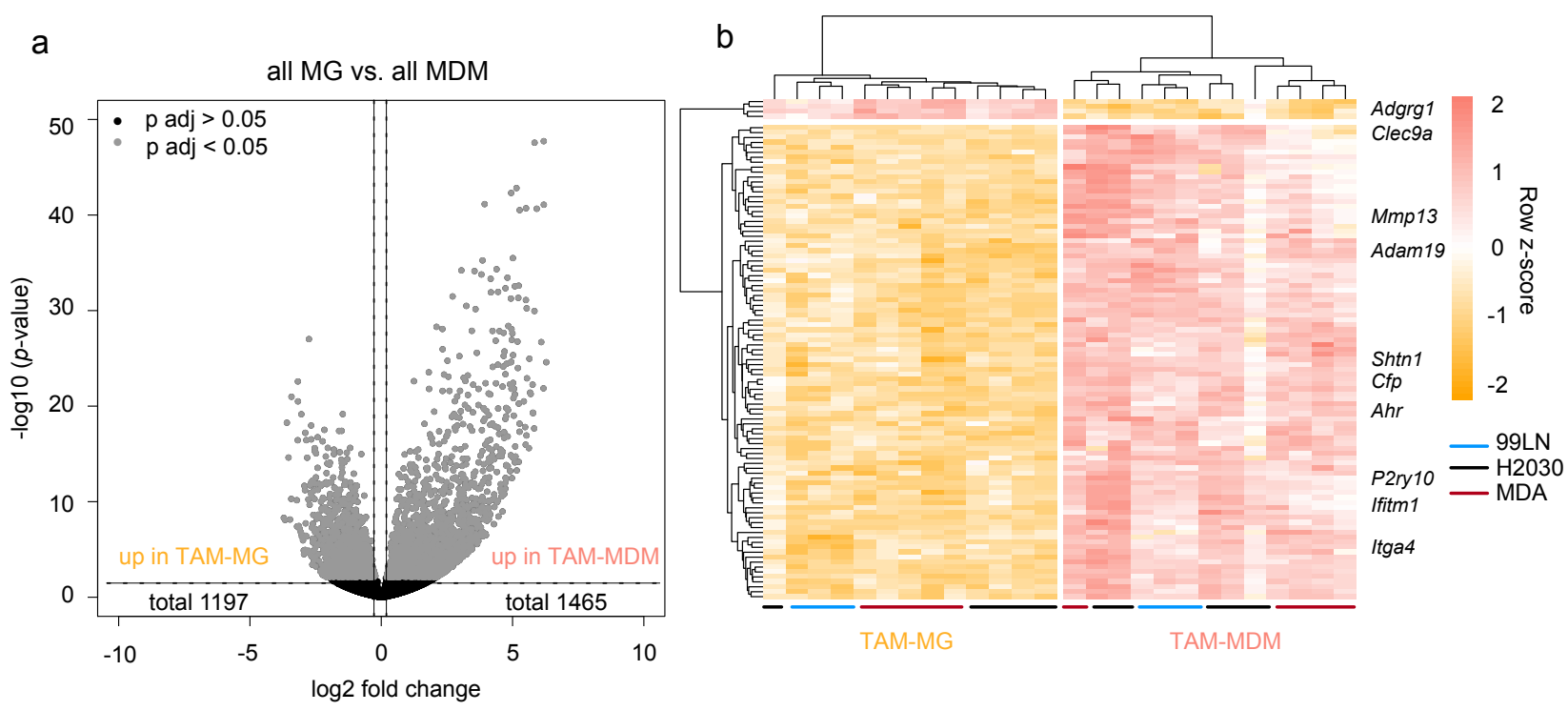

C

regulation of cell morphogenesis

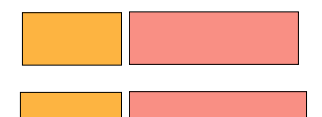

TAM-MDM

Arachidonate production from DAG

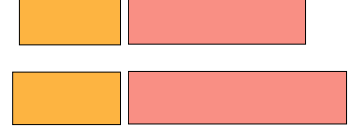

second messenger-mediated signaling

glial cell migration cell morphogenesis involved in
Neuron differentiation

lipid biosynthetic process

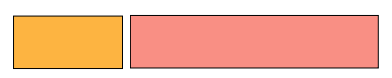

regulation of cytokine production

response to IFN gamma

ameboidal-type cell migration

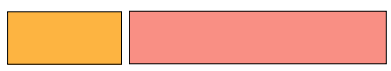

regulation of peptide

positive regulation of immune response

pos. regulation of cell death

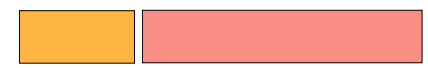

regulation of defense response

neutrophil degranulation

cell-cell adhesion

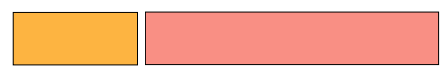

inflammatory response

glycoprotein biosynthetic process

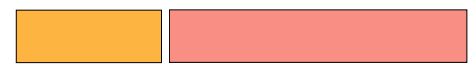

T cell activation

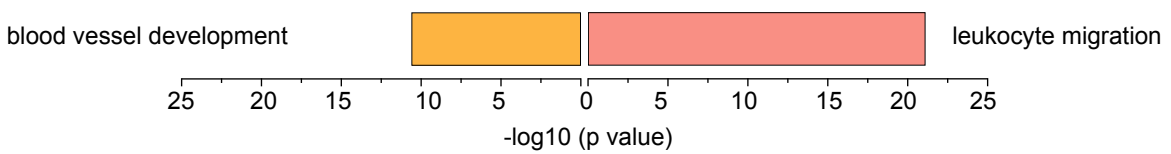

Figure 23: TAM-MGs and TAM-MDMs represent two distinct macrophage populations in BrM. a) Volcano plot of the comparison from all TAM-MGs vs. all TAMMDMs derived from the three different models. Each dot represents one gene, either significantly regulated between both cell types (grey), or not (black). b) Unsupervised clustering heatmap of the Top 100 DEGs between all TAM-MGs vs. all TAM-MDMs derived from the three models. c) Functional annotation and pathway analyses of all DEGs upregulated in TAM-MGs (left) or TAM-MDMs (right). Note, that some or all of the data belonging to H2030-BrM (Schulz et al., 2020), MDA-BrM (Klemm et al., in revision), and 99LN (Niesel et al., 2021) have been published before and are partially modified. 
Because macrophages are traditionally classified into M1 (pro-inflammatory) or M2 (anti-inflammatory and pro-tumorigenic) polarization states, a panel of M1 and M2 markers across both TAM populations in each model was examined (figure 24a,b). RNA-Seq data indicated a rather mixed polarized phenotype of both TAM-MG and TAM-MDM, since several M1 but also M2 markers were similarly up- or downregulated across all models (figure 24a,b). The majority of examined M1 marker was upregulated, and partially highly significant in both TAM populations. Among them were distinct chemokines (e.g. Cc/2, Cc/3, Cc/4, Cc/12), interleukins (II1b, I/12b), Socs3, and Tnf. Adversely regulated (down in TAM-MG, up in TAM-MDM) were $C D 80$, II6, and TIr4. Several M2 markers showed a higher diversity in regulation, both within and between cell types (figure 24a,b). Most apparent was the fact that some traditional M2 markers were rather upregulated in TAM-MDM, and only slightly regulated in TAM-MG (e.g. Arg1, Cc/17, Cc/22, Ccl24, T/r1). Other genes were only slightly, or apparently downregulated like Tgfb1. In addition, there was a relatively unique upregulation of Igf1, $/ 110$, and Mrc1 in both TAM populations, whereas the latter one was surprisingly only downregulated in 99LN-TAM-MG, hence most likely also useful as a marker for IF as shown before (figure 16). Chil3, another classical M2 marker in murine macrophage biology, was or tended to be rather downregulated in both TAM populations (figure 24b). In summary the data suggest that both, TAM-MG and TAM-MDMs possessed mixed polarization phenotypes on the population-wide level across different BrM models. This finding was further mimicked by in vitro stimulation experiments with cancer cell-derived supernatant applied to EOC2 microglia, or primary BMDMs. As an example a set of two genes for every polarization state of EOC2 microglia and BMDMs stimulated with 99LN supernatant is shown (figure 24c). While Tnf was similarly regulated in vitro and in vivo in MGs, Ifnb1 was not and showed lower expression levels in vitro upon stimulation with 99LN supernatant (figure 24c). Two M2-associated markers (Cc/17, Cc/22) were only slightly de-regulated compared to controls (figure 24c), similar to the in vivo TAM-MG profile (figure 24b). BMDM expression profiles were similar in vitro. While Ifnb1 was slightly higher in vivo, BMDMs showed apparent lower expression upon stimulation in vitro. Comparable to the in vivo situation was the dramatic upregulation of the M1 marker $1112 b$. More surprisingly, both M2 markers showed no expression changes in the in vitro setting, contradictory to the highly significant upregulation in vivo. 
Together the data clearly indicate a certain masking of individual cell profiles, since previous immuno-histologic, live imaging, and single-cell approaches suggest a very dynamic, heterogeneous TME, especially in the TAM population. Comparison of in vitro with in vivo data further reveals the complexity of the TME in which different stimuli modulate gene expression of TAMs. This complexity cannot be fully recapitulated in vitro.

a

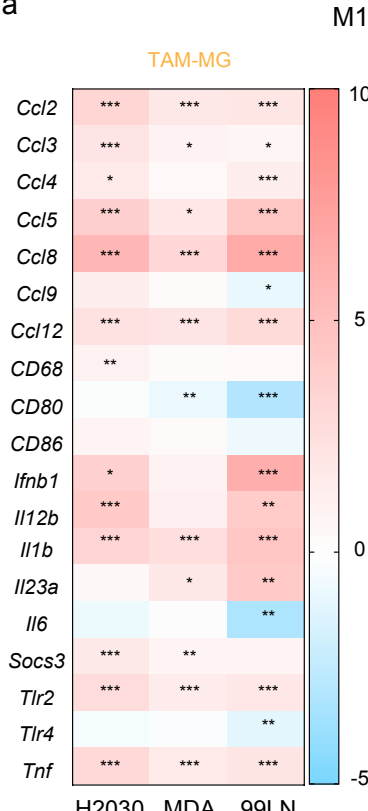

H2030 MDA 99LN

C

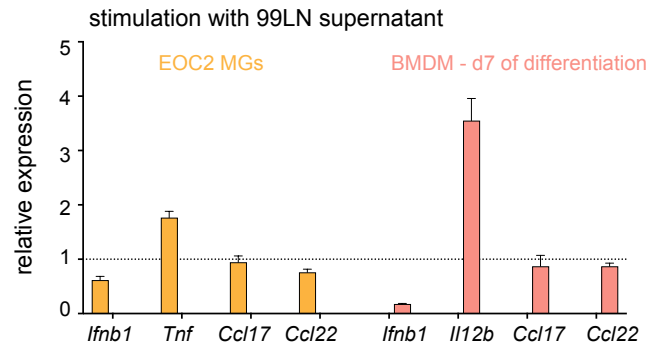

b
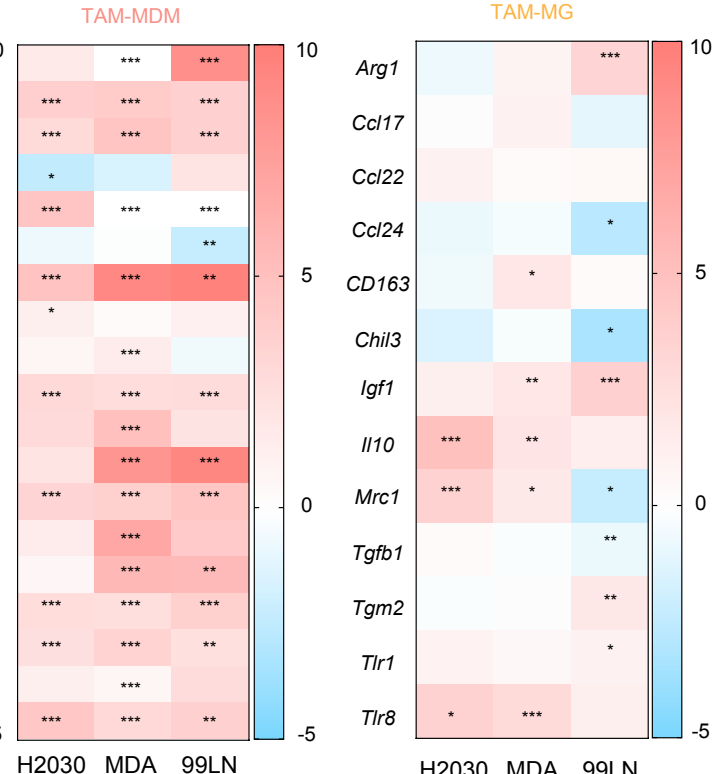

H2030 MDA 99LN
M2

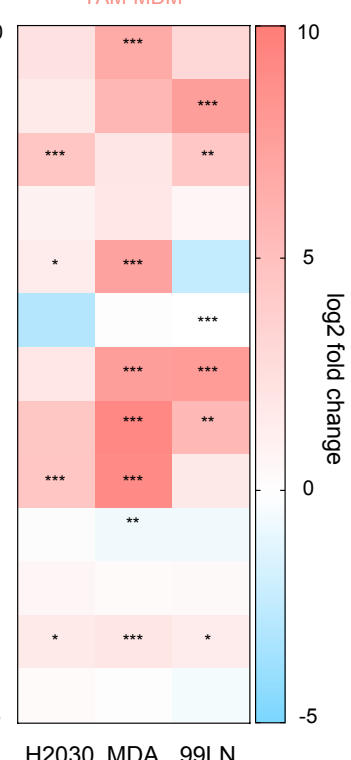

Figure 24: M1 and M2 polarization marker across both TAM populations in different BrM models. a) Heatmap depicting log2 fold change of indicated genes associated to M1 polarization; represented is the BrM situation (TAM) as compared to the normal cellular counterpart. Significance derived from padj. values with $p<0.05\left(^{*}\right), p<0.01$ $\left.\left({ }^{* *}\right), p<0.001{ }^{* * *}\right)$. Empty cells were not significant or no statistical data were available. b) Heatmap depicting log2 fold change of indicated genes associated to M2 polarization; represented is the BrM situation (TAM) as compared to the normal cellular counterpart. Significance derived from padj. values with $p<0.05\left(^{*}\right), p<0.01$ $\left({ }^{* *}\right), \mathrm{p}<0.001{ }^{\left({ }^{* *}\right)}$. Empty cells are not significant or no statistical data were available. c) Relative expression of indicated genes from stimulation experiments of EOC2 microglia or BMDMs at d7 with supernatant from 99LN cells in vitro. Line at 1 represents the control condition of unstimulated cells, of which values were normalized to EOC2: all genes from $n=3$ biological replicates, BMDM: $n=3$ biological replicates, apart from Cc/17 $(n=2)$. Note that some or all of the data belonging to H2030-BrM (Schulz et al., 2020), MDA-BrM (Klemm et al., in revision), and 99LN (Niesel et al., 2021) have been published before and are partially modified. 


\section{E) The Complement system in BrM}

1. The complement system as a central mediator of BrM-associated inflammation The majority of previous analysis revealed members of the complement system being present among the top upregulated genes. In preclinical and clinical data of neurodegenerative and -inflammatory diseases, complement activation has been shown to exert central functions with the involvement of brain-resident cells like microglia and astrocytes (Reis et al., 2019). However little is known in the BrM situation, hence RNA-Seq data of all TAMs and TILs were queried for the expression changes of certain complement members within all models (figure 25).

Interestingly, there were several complement components and respective receptors consistently similarly regulated in both TAM populations. $C 1 q b$ and $C 1 q c$ for example were significantly upregulated in TAM-MG of H2030 (Schulz et al., 2020) and MDA, but not in 99LN, indicating a background-specific response. On the other hand, C2 was significantly upregulated in TAM-MG of MDA and 99LN, but not H2030, arguing for a specific type of response towards B2B metastasis. Consistently and highly significant was the upregulation of $C 3$ and $C 4 b$ in TAM-MG derived from each of the three models (figure 25a). C5ar1, a C5 receptor, was significantly upregulated in TAM-MG from H2030, MDA, and 99LN, as well (figure 25a). Several other members and receptors were similarly upregulated in TAM-MDM derived from all models (figure 25b). Among them were C1qa, C1qb, C1qc, C4b, and the receptors C3ar1, and C5ar1. Interestingly, B2B-specifically upregulated were C5ar2, and similar to TAM-MGs, C2. In order to prove the gene expression results independently from RNA-Seq, the upregulation of $\mathrm{C} 3$ and C3ar1 expression was validated in sorted TAMs from $\mathrm{H} 2030$ via qPCR compared to their normal cellular counterparts (figure 25c) (Schulz et al., 2020). In addition, enhanced protein abundance of $C 1 Q, C 3$ and C3AR1 was confirmed in H2030-associated TAMs via IF (figure 25d). Multi-factor staining furthermore revealed the abundance of $\mathrm{C} 3 \mathrm{AR} 1^{+} / \mathrm{IBA} 1^{+}$as well as $\mathrm{C} 3^{+} / \mathrm{IBA} 1^{+}$ cells within H2030-BrM. Several IBA $1^{+}$cells however showed no signal of C3AR1, and inversely cells positive for C3AR1, but without IBA1 signal populated BrM (figure 25e).

Looking beyond the myeloid compartment of BrM, TILs from the 99LN model showed only low expression of complement members, with the exception of three C1q members, namely C1qa, C1qb, and C1qc (figure 25f). While they showed a high but 
stable expression among control or TIL B cells, most of them were absent in both control T cell populations, but significantly elevated their expression in the context of BrM (figure 25f).

Because distinct complement members were similarly regulated within TAMs of different models, the influence of WBRT on the expression levels of these genes was further examined. Hence, H2030-associated TAM data were queried for some of the above-mentioned complement members (figure $25 \mathrm{~g}$ ). Surprisingly, the overall trend showed no or only slight alterations among the expression levels of genes of interest in TAM-MG under treatment-naïve or treated conditions. Most obvious alterations included samples within the expression of $C 3, C 4 b$, and $C 5$. Samples derived at d10 following $5 \times 2 \mathrm{~Gy}$, and a single high dosage used for WBRT (d3 upon $1 \times 10 \mathrm{~Gy}$ ) resulted in slightly higher expression levels of $C 3$, and $C 4 b$ in TAM-MG (figure 25g). Very apparent was the massive downregulation of $C 5$ in TAM-MG irradiated once with $10 \mathrm{~Gy}$, which its biological relevance requires further examination. The TAMMDM compartment showed similar consistency across different conditions. Only C3 and C5 showed greater alteration, and an overall lower expression in TAM-MDM of $\mathrm{H} 2030$-bearing mice irradiated once with $10 \mathrm{~Gy}$ (figure 25g).

In summary the data show that complement expression of $\mathrm{H} 2030$-associated TAMs lacked changes in response to different regimens of WBRT. Hence the instruction of TAMs via tumor cells is sufficient for the expression of distinct complement members, regardless of the applied treatment modalities. 
VI Results

a

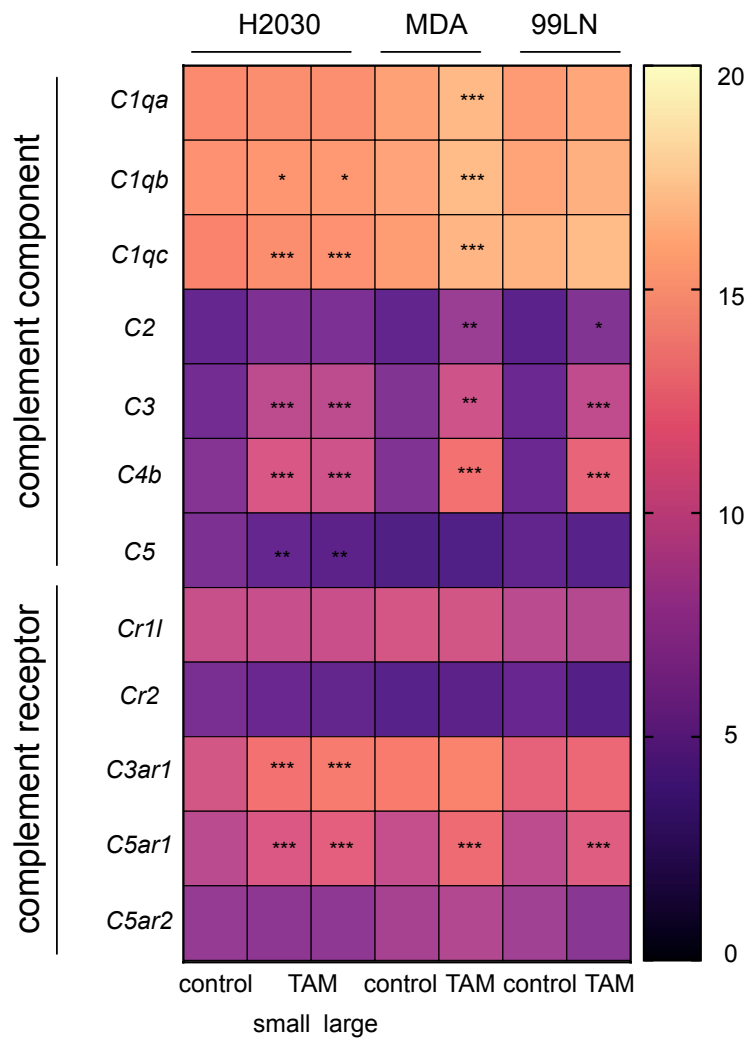

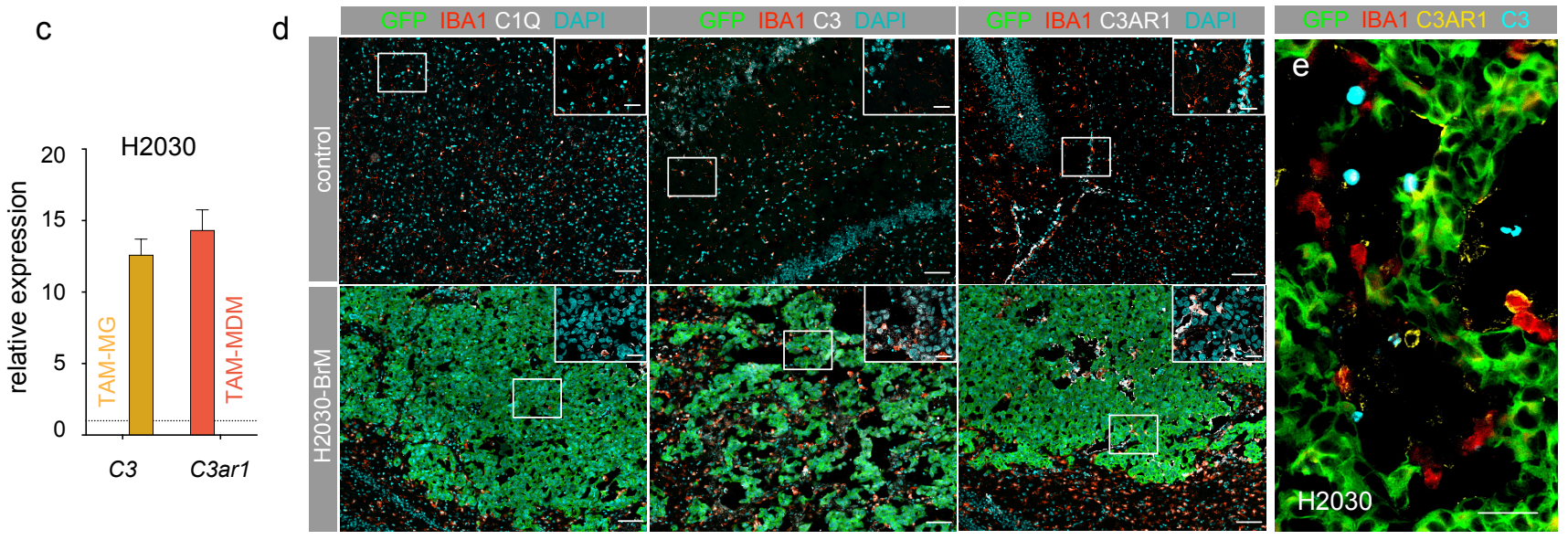

f

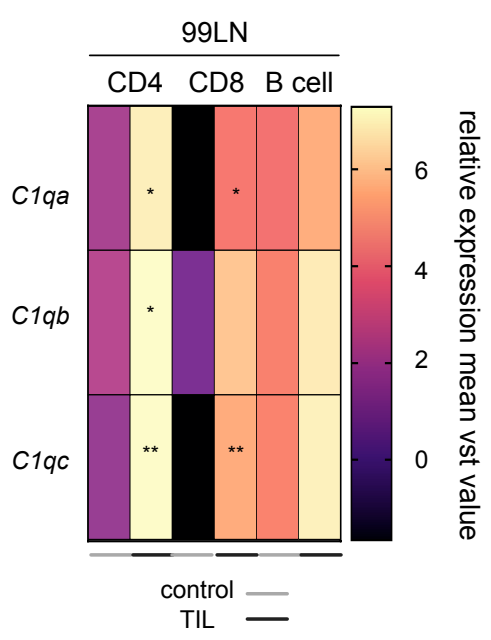

b

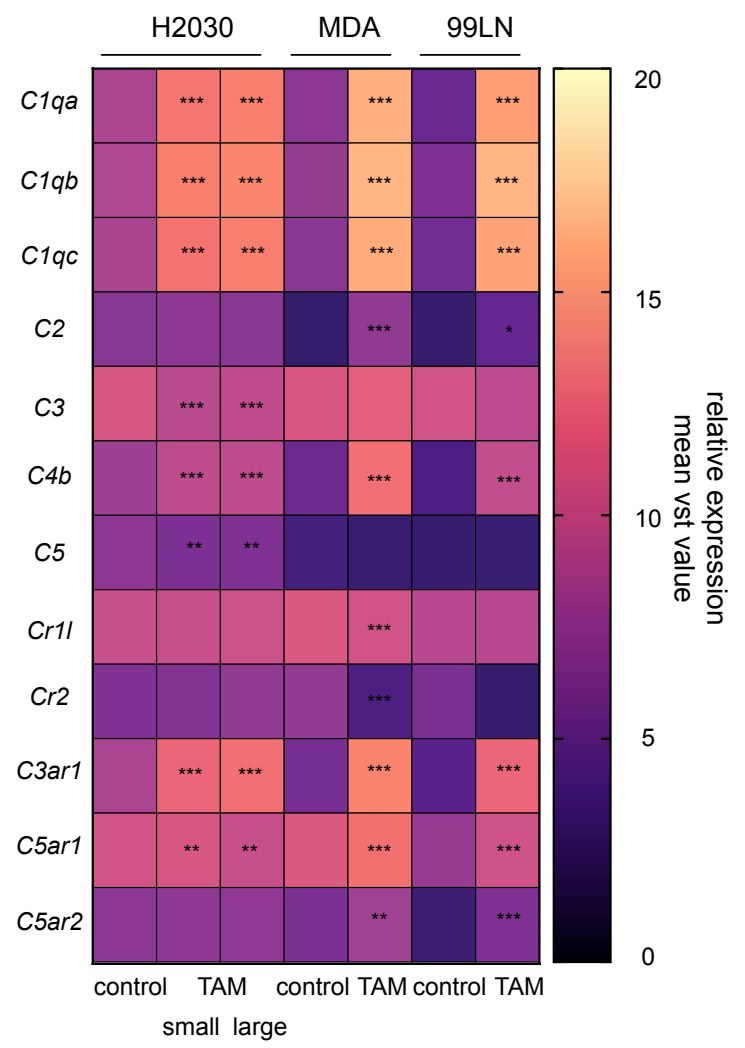

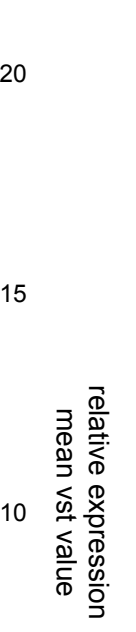

g $20, \mathrm{H} 2030$

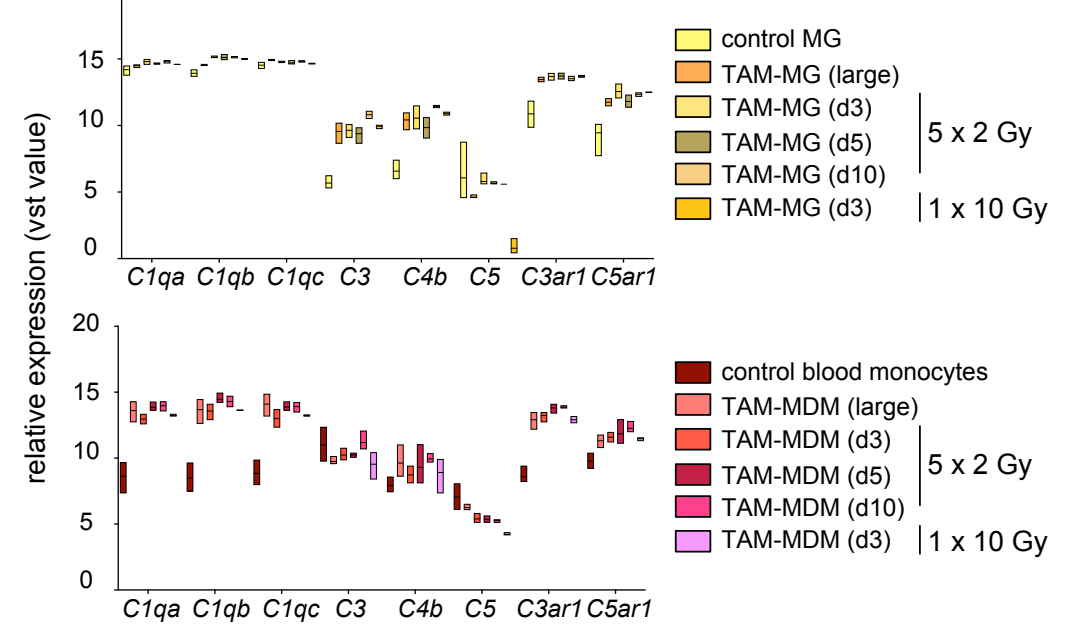


Figure 25: The complement system in murine BrM-associated immune cells.

a) Heatmap depicting mean vst value as relative expression levels per condition for some indicated genes belonging to members of the complement system in TAM-MG. Data derived from control vs. TAM comparison with $\mathrm{n}=3-5$ per condition and model. Significance derived from padj. values with $p<0.05\left({ }^{*}\right), p<0.01\left({ }^{* *}\right), p<$ $\left.0.001{ }^{* * *}\right)$. Empty cells were not significant or no statistical data were available. b) Heatmap depicting mean vst value as relative expression levels per condition for some, indicated genes belonging to members of the complement system in TAMMDM. Data derived from control vs. TAM comparison with $n=3-5$ per condition and model. Significance derived from padj. values with $p<0.05\left(^{*}\right), p<0.01\left(^{* *}\right), p<$ $\left.0.001{ }^{* * *}\right)$. Empty cells were not significant or no statistical data were available. c) Validation of two complement member genes by qRT-PCR of sorted TAM-MG or TAM-MDM samples out of H2030-BrM. Data are represented as relative expression to 1 (dotted line), which was set for control cells (normal microglia or blood monocytes). Data are shown as mean of $R Q$ values $+R Q$ max, and data are derived from $n=2$ independent biological replicates. d) Representative IF stainings of some members of the complement system (white) in control slides (upper row) vs. treatment-naïve H2030-BrM slides (lower row). GFP (green) was used for tumor cell staining, and IBA1 (red) for TAM staining. DAPI (blue) was used as nuclear counterstain. Scale bars $=100 \mu \mathrm{m}$ and inlays $=25 \mu \mathrm{m}$. e) Representative IF staining of several complement members within a close-up of $\mathrm{H} 2030-\mathrm{BrM}$. DAPI could not be used as nuclear counterstain since max. colors for image acquisition was reached. Scale bar $=200 \mu \mathrm{m}$. f) Heatmap depicting mean vst value as relative expression levels per condition for some, indicated genes belonging to members of the complement system in TILs from 99LN-BrM. Data derived from control vs. TIL comparison with $n=3$ per condition. Significance derived from padj. values with $p<$ $0.05\left({ }^{*}\right)$, or $p<0.01\left({ }^{* *}\right)$. Empty cells were not significant or no statistical data were available. g) Relative expression level (vst values) of certain complement components and receptors from $\mathrm{H} 2030-\mathrm{BrM}$ associated microglia and macrophages within distinct control and irradiated conditions as indicated. Data include $n=3$ for $d 3$ ( $1 \times 10 \mathrm{~Gy})$ and d10 (5 x 2 Gy), n = 4 for control MG, and TAM-MG d3 and d5 (5 x 2 Gy), $n=5$ for TAM-MG (large, no IR). Note that some or all of the data belonging to H2030-BrM (Schulz et al., 2020), MDA-BrM (Klemm et al., in revision), and 99LN (Niesel et al., 2021) have been published before and are partially modified. 
2. The complement system in human BrM-associated microglia and macrophages

In order to translate the findings to the clinical situation, expression changes of complement members in TAMs of human BrMs were analyzed based on a previously published data set (Klemm et al., 2020). Initially, patients were stratified according to the primary tumor their BrMs originate from and the following samples of bulk RNASeq data were combined and relative expression changes were plotted (figure $26 a, b)$. Both control conditions included seven samples, derived from blood of healthy donors or MG from epilepsy brains. The breast-to-brain (B2B) cohort included patients \# $3,7,10$, and 20 , and most of the samples were derived from recurrent BrMs. The lung-to-brain (L2B) cohort included patients \# 11, 13, 39, and 50. All samples are derived from initial BrMs of NSCLC (non-small cell lung cancer, adenocarcinoma). Additionally, samples from recurrent NSCLC BrMs were available (patient \# 2, 8, 32, 47) and included (figure 26b). Of note, samples from the recurrent cohort were derived from patients that underwent any kind of therapy before (figure 26b). Interestingly, all genes examined were not or only very slightly regulated compared to the normal control situation in the MG compartment derived from B2B patients (figure 26a). C1QA, C1QB, C1QC, C3, C5, C5AR1 showed no apparent differences in expression levels, whereas $C 4 B$ was slightly up-, and $C 3 A R 1$ slightly downregulated (figure 26a). In the TAM-MDM population however, several genes showed a similar regulation to MDMs within the murine BrM models. Clearly upregulated were $C 1 Q A, C 1 Q B, C 1 Q C, C 3$, and $C 3 A R 1$. All of them apart from $C 3$ (figure 26a) were upregulated in murine B2B TAM-MDM as well (figure 25b). Contrary, TAM samples from L2B metastases partially showed different expression pattern to the human B2B, and murine H2030 samples. Interestingly, TAM-MG of initial $L 2 B$ tended to downregulate all markers of interest, including $C 1 Q A, C 1 Q B$, $C 1 Q C, C 3$, and C3AR1. Contrary, TAM-MDM of initial L2B upregulated C1QA, $C 1 Q B, C 1 Q C$, and C3AR1, whereas $C 3, C 4 B, C 5$, and C5AR1 did not show any expression changes (figure 26a). Making use of the available data from recurrent L2B metastases, these samples were separately included as well. Genes analyzed in both, TAM-MG and TAM-MDM, showed similar expression levels in recurrent samples than initial ones, apart from C4B in TAM-MG. This was the only condition different to the initial L2B group, and expression changes revealed a higher mean expression level (figure 26a). 
Collectively, the snapshot of human data shows differences but also similarities between mouse and human complement gene expression levels in TAMs. Interestingly, there were also several differences in BrMs derived from various primary tumor types, i.e. lung vs. breast-to-brain.

human brain TAM data

a

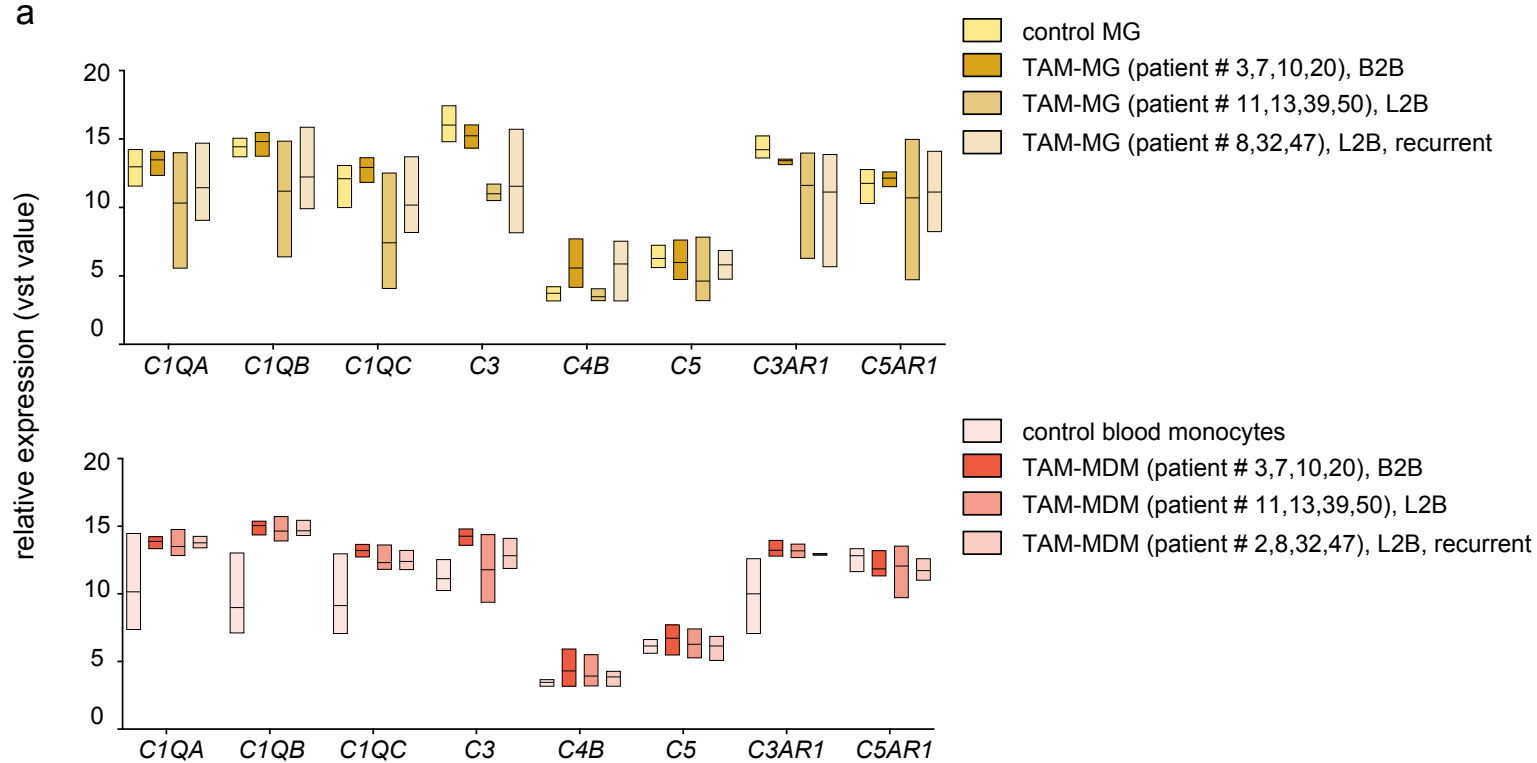

b

\begin{tabular}{|c|c|c|c|c|c|}
\hline atient & primary & status & radiotherapy ch & emotherap & therap \\
\hline$\# 3$ & breast & recurrent & no & no & no \\
\hline \#7 & breast & recurrent & $>6$ month & $>6$ month & no \\
\hline$\# 10$ & breast & recurrent & no & NA & no \\
\hline \#20 & breast & NA & NA & NA & no \\
\hline$\# 11$ & $\begin{array}{l}\text { lung, NSCLC } \\
\text { adenocarcinoma }\end{array}$ & initial & no & no & no \\
\hline$\# 13$ & $\begin{array}{l}\text { lung, NSCLC } \\
\text { adenocarcinoma }\end{array}$ & initial & no & no & no \\
\hline$\# 39$ & $\begin{array}{l}\text { lung, NSCLC } \\
\text { adenocarcinoma }\end{array}$ & initial & no & no & no \\
\hline$\# 50$ & $\begin{array}{c}\text { lung, NSCLC } \\
\text { adenocarcinoma }\end{array}$ & initial & no & no & no \\
\hline \#2 & $\begin{array}{l}\text { lung, NSCLC } \\
\text { adenocarcinoma }\end{array}$ & recurrent & $\begin{array}{c}>6 \text { month } \\
\text { (SRS other lesion) }\end{array}$ & NA & no \\
\hline \#8 & $\begin{array}{l}\text { lung, NSCLC } \\
\text { adenocarcinoma }\end{array}$ & recurrent & no & $>6$ month & no \\
\hline$\# 32$ & $\begin{array}{l}\text { lung, NSCLC } \\
\text { adenocarcinoma }\end{array}$ & recurrent & $>6$ month & $<3$ month & no \\
\hline$\# 47$ & $\begin{array}{l}\text { lung, NSCLC } \\
\text { adenocarcinoma }\end{array}$ & recurrent & no & $>6$ month & no \\
\hline
\end{tabular}

Figure 26: The complement system in human BrM-associated macrophages and microglia. a) Relative expression level of some selected complement members in TAMs from human patient BrM. $\mathrm{N}=7$ for controls, MG (upper) and blood monocytes (lower). b) Clinical characteristica of human samples. TAM data derived from different patients and stratified according to primary tumor type, both as indicated. Data derived from Klemm et al. (2020), and accessed via https://joycelab.shinyapps.io/braintime/. 


\section{Discussion}

A) Different types of brain metastasis vary in their immune cell infiltration pattern

The cellular landscape is the basis of organ integrity, hence indispensable for its homeostatic functions. While the brain traditionally has always been regarded as immune-privileged, recent studies provided accumulating insight into its complex cellular immune environment under homeostatic conditions (e.g Korin et al., 2017, Mrdjen et al., 2018). In addition, several recent studies characterized the immune landscape in the context of different neuro-degenerative, neuro-inflammatory, and malignant diseases, including brain metastasis.

In order to evaluate the impact of BrM induction and progression on its TME, different cellular approaches including histology and flow cytometry were employed within this thesis. While responses of non-immune, brain-resident cell types towards BrM were not further examined herein, their similarity between preclinical models and the clinical situation suggests conservative mechanisms relevant for the induction and maintenance of reactive phenotypes, e.g. astrogliosis. A major contributor for assembling pro-tumorigenic milieus are astrocytes (Wasilewski et al., 2017), which even recognize a few single tumor cells and become reactive, as observed in live cell imaging experiments during this work. However, since they are an integral part of the BBB their contribution might even start before tumor cells extravasate into the parenchyma, thereby also regulating interaction with other cells from the periphery, like monocyte-derived macrophages (MDMs). Previous studies reported a substantial contribution of MDMs to the local TAM pool in BrM, overall representing one of the main stromal cell types within the whole tumor mass (Sevenich et al., 2014, Sevenich 2018). However in order to elucidate BrM stage- and background-specific states, results of this work suggest analogies of TAM-MDM recruitment. On one hand, all models examined here constantly recruit TAM-MDMs during tumor progression, but on the other hand to a very different extent. Likewise recent analyses of human BrM samples unraveled substantial differences of TAM-MDM recruitment, based on the primary origin (Friebel et al., 2020, Klemm et al., 2020). Despite the fact that different treatment modalities of BrM patients examined in the human studies might influence the recruitment behavior of peripheral immune cells into BrM, L2B metastases showed the highest amounts of TAM-MDMs as seen in the L2B model H2030-BrM (Schulz et al., 2020). Moreover, the monocyte-derived cell infiltration in different 
backgrounds of glioma models, in which TAM-MDMs contributed between $10-40 \%$ to the whole TAM population (Bowman et al., 2016, Akkari et al., 2020), argues for background-specific responses. Future work needs to elucidate the functional contribution and trafficking routes of granulocytes/neutrophils into BrM. The entirety of immune cells might even infiltrate through different ways, whereas routes via blood and/or lymphatic vessels seem more traditional. In addition, the CSF might act as a fast way of recruitment, as it has been shown to harbor basically every immune cell type in patients with MS (Schafflick et al., 2020). In addition another very recent study showed that CSF analyses recapitulated brain inflammation. Moreover, TCR genotyping revealed overlapping sequences between T cells from either CSF or BrM, indicating cellular exchange between both compartments (Rubio-Perez et al., 2021). Although there were hardly any granulocytes detected in the melanoma model (H1_DL2), L2B (H2030-BrM) and B2B (99LN-BrM) metastasis induced the infiltration of a substantial amount of granulocytes especially at late stages. However this effect might also be driven by the strong bleeding and hemorrhage frequently seen in large H2030 BrM, and L2B metastases in general. Neutrophils represent one of the most abundant non-TAM populations in human BrM, as well (Klemm et al., 2020). Given the fact that they might also infiltrate directly from skull bone marrow via connecting channels to the parenchymal surface (Herisson et al., 2018), their role in outgrowing lesions seems a feasible subject to investigate in future studies since they probably belong to the first responders upon BrM initiation. Transcriptomic profiles of neutrophils infiltrating human BrM suggest their participation in regulating immunesuppression and recruitment of other immune cells (Klemm et al., 2020), including T cells. As in the human situation (Harter et al., 2015, Friebel et al., 2020, Klemm et al., 2020), the lymphoid compartment in B2B metastasis (99LN-BrM) is mainly composed of T-lymphocytes, which relative numbers negatively correlate with tumor size. Contrary, B cells and NK cells are less abundant (this work, and Niesel et al., 2021). Interestingly, the major subsets of $\mathrm{T}$ cells $\left(\mathrm{CD} 4^{+}\right.$and $\left.\mathrm{CD} 8^{+}\right)$do not change their relative contribution to the T cell population during BrM progression as seen in 99LN$\mathrm{BrM}$, indicating BrM- or BrM-TME-dependent regulatory mechanisms of cell infiltration. However the depletion of $\mathrm{CD}^{+}$and $\mathrm{CD}^{+} \mathrm{T}$ cells does not influence $\mathrm{BrM}$ onset or progression within 99LN-BrM (Niesel et al., 2021). Human samples of primary and metastatic brain tumors also showed similar relative frequencies among different TIL subsets, regardless of the primary origin, assuming a mixed bulk 
population of patient samples and frequencies observed in TILs in BrM were negatively correlated with the number of TAMs (Friebel et al., 2020). Additionally involved within this interplay are most likely not only tumor cells themselves, but rather every other cell type in the BrM TME. Consistent with these assumptions are further previous publications highlighting the involvement of astrocytes (Priego et al., 2018) and MDMs, together all contributing to the development of an immunosuppressive environment (Quail and Joyce, 2017, Schulz et al., 2019, Friebel et al., 2020, Klemm et al., 2020, Schulz et al., 2020, Niesel et al., 2021). Localization of macrophages in close proximity to TILs however further shows potential for the induction of adaptive immune responses towards BrM, which evidently are not sufficiently utilized (Klemm et al., 2020).

Not only do different primary tumor entities induce their own specific immune cell landscape, but also show distinct anatomical preferences to grow out. Melanoma BrM were shown to significantly accumulate rather in frontal locations of patients' brains, whereas B2B metastasis showed preferences for the establishment in the cerebellum (Schroeder et al., 2020). Cellular (e.g. distinct types of neurons, specific subsets of astrocytes) and molecular (e.g. cytokines) differences in the microenvironment might be responsible for that. The excrescence of BrM itself varies between angiogenic (lung-to-brain) or co-optive growth pattern (melanoma-to-brain) (Kienast et al., 2010). In addition this most likely plays an essential role and recent findings underlining early observations (Stevens et al., 1988) showed that various primaries also induce variable pattern of infiltrative immune cells (e.g. MDMs) (Friebel et al., 2020, Klemm et al., 2020). The same is true for the growing body of knowledge on immune infiltrates under neuro-inflammatory conditions like Multiple Sclerosis (MS), or its pre-clinical equivalent EAE (Jordao et al., 2019, Schirmer et al., 2019). Important to note here are lymphatic vessels which just recently have been rediscovered in the brain and have been shown to drain brain tumor antigens, thereby being involved in adaptive immunity of malignancies to the brain (Song et al., 2020). Hence it is likely that tumor cells themselves already pave the way to their specific future TME by intrinsic traits, like the secretion of high amounts of distinct myeloid cell-attracting chemokines in L2B metastasis for instance (Schulz et al., 2020). Moreover variable growth pattern additionally harbor the possibility to influence the recruitment of immune cells via a non-intact BBB/BTB with heterogeneous permeability (Arvanitis et al., 2019). 
Due to the abovementioned facts it is becoming evident that the induction and maintenance of BrM-TMEs is a very complex, multi-variate process, involving plastic interactions of/with: different primary tumor types, their pattern of outgrowth, the location they grow in, the specific environment of brain-resident cells, the molecular environment (e.g. cytokines), and probably even, specific individual traits (e.g. other molecular pattern or mutations in certain genes of TME-associated cells) and habits (e.g. physical activity (Lowe et al., 2014)). Hence evaluation of immune infiltrates always has to be performed in a context-dependent manner. Apart from analyzing the cellular changes in the BrM TME a major focus within this thesis was the examination of molecular alterations related to BrM-associated immune cells upon instruction by tumor cells of different origin.

B) Transcriptomic profiles of the tumor microenvironment in brain metastasis

\section{Lymphocytes become exhausted within the BrM TME}

While fully immuno-competent mouse models of BrM are rare, the 99LN-BrM represents a very robust model, recapitulating traits of human B2B metastasis (Bowman et al., 2016, Chae et al., 2019). Although there are differences in recruitment behavior of $\mathrm{T}$ cells during metastatic outgrowth, tumor progression itself has been shown to have very little influence on BrM-associated inflammatory cell transcriptomes, at least in the myeloid compartment (Schulz et al., 2020). Hence, regardless of the BrM stage, RNA-Seq of TILs isolated from 99LN-BrM with an average size of about $50 \mathrm{~mm}^{3}$ (not shown here) has been performed (Niesel et al., 2021). Although bulk sequencing most likely fails to detect specific subpopulations of lymphoid effector cells, the overall trend of expression changes is consistent with recent data from human BrM in both, bulk RNA-Seq data (Klemm et al., 2020) and protein abundance (Friebel et al., 2020). Most notably is the upregulation of markers associated to $\mathrm{T}$ cell activation and exhaustion, which represents a typical phenomenon in cancer (Davoodzadeh Gholami et al., 2017). CD4 ${ }^{+}$and CD8 ${ }^{+}$TILs of human BrM were found to be rather anergic, showing exhausted phenotypes (Friebel et al., 2020, Klemm et al., 2020). As example, 99LN-associated TILs upregulated typical marker like Ctla4, Granzyme K, Helios, Ifng, Lag3, PD-1, and Tnf. Although only slightly and especially in $\mathrm{CD}^{+} \mathrm{T}$ cells, the upregulation of the major transcription factor driving $\mathrm{T}$ cell activation suggests a Tox-driven regulatory network resulting in $\mathrm{T}$ 
cell exhaustion, as previously shown for TILs (Scott et al., 2019). Together, these data argue for the suitability of the 99LN-BrM model in order to study different aspects of BrM-associated inflammatory responses on the molecular level. Since there is a massive decline of TIL numbers during B2B metastases progression, in future studies it might still be relevant to identify molecular profiles which are rather associated to very early stages of this subset of BrM. In case of fundamental differences between early and late stage TIL transcriptomes, this identification would allow the design of early interventional targeted therapies, like immune checkpoint blockade (ICB) as adjuvant therapy option to IR. Recent insight revealed that checkpoint inhibition in combination with radiation has the potential to attenuate BrM progression within the 99LN model (Niesel et al., 2021), which clearly suggests the critical need of evaluation of targeted-therapies simultaneously applied with standard of care. In addition, this study further suggests to focus on lifting immunesuppression coming from the myeloid compartment.

\section{Microglia and macrophages represent two distinct populations in BrM}

Within the brain cancer setting, several studies in the past defined the TAM population as one homogeneous cell population without further distinguishing them based on their ontological origin. The lineage-tracing-based identification of CD49d, which delineates brain-resident microglia from monocyte-derived macrophages (MDM) (Bowman et al., 2016) however has tremendously shifted our view on the diverse pool of TAMs in brain cancer. Since TAMs in various tumor settings increasingly gained attention for the development of targeted therapies, TAMs in brain tumors have become of particular interest due to their high abundance, but also great plasticity in promoting tumor biology (Quail and Joyce, 2017, Sevenich, 2018, Schulz et al., 2019). Upon more detailed investigation and description of dichotomy of both major TAM populations in primary brain tumors (i.e. glioma) of preclinical models (Bowman et al., 2016, Chen et al., 2017), the publication derived during this thesis (Schulz et al., 2020) was the first one to show the transcriptional heterogeneity of TAMs in BrM. These findings were underlined by much deeper molecular profiling of TAMs from human BrMs shortly after (Friebel et al., 2020, Klemm et al., 2020). In sum, all of these data show great phenotypic heterogeneity among brain TAMs. Very interestingly, pseudotime trajectory analysis of different human brain TAM-MDMs revealed that each tumor entity, both primary and secondary, induces the 
development of its own heterogeneous MDM population with distinct tumor typespecific signatures (Friebel et al., 2020). These data argue for the great conditiondependent plasticity TAMs exhibit in the brain tumor setting. Similarly, cross model comparison of mouse TAM signatures (figure 21) showed specific set of genes related to different conditions (e.g. background or primary tumor entity), and great plasticity of polarization phenotypes in TAMs (figure 24), similar to the human situation (Klemm et al., 2020). It seems contradictory that on the other hand sets of genes appear equally regulated in the BrM situation, indicative of a core BrM-specific signature. Together these sets of similarly regulated genes will be of critical importance for further examination in order to identify specific BrM-driven molecular pattern. Microglia associated to any kind of BrM for example showed distinct pattern related to TNF or IFN signaling, which however seem to be "common" genes enriched in diseased microglia (Friedman et al., 2018). Hence in order to identify "real" BrM-specific pattern, additional in silico analyses and comparison on the single cell level are required.

Since another recent study even suggested BAMs to contribute to the macrophage pool in BrM (Guldner et al., 2020), it remains to be shown which molecular and consequently functional differences evolve due to the different origins of macrophages contributing to the local TAM-MDM pool. In contrast to the infiltration from the periphery, local brain-resident TAM-MG clearly segregate from the TAMMDM population on the molecular level (figure 21, 22). Although infiltrating MDMs under neurodegenerative conditions do not acquire MG signatures (Butovsky et al., 2014), MDMs following BrM education slightly upregulated some of their markers (e.g. P2ry12, Tmem119), whereas TAM-MG in mouse (shown here) and human BrM (Klemm et al., 2020) downregulate homeostatic markers. However, this does not seem to affect the overall differences on the functional level. The annotation of gene sets from both TAM populations remarkably uncovers conservative (across models and species) variations in the BrM situation: while macrophage functions seem to be associated to wound healing responses, ECM modulation, antigen presentation and interaction with the T cell compartment, the brain TAM-MG core gene set is rather related to pro-inflammatory functions (Bowman et al., 2016, Klemm et al., 2020, Schulz et al., 2020, Niesel et al., 2021) and likely involves the interaction with other resident (Priego et al., 2018) or recruited cell types. The exact function and potential other mediators influencing the TME with regards to the lymphoid compartment need 
further examination. Since the increase of TAM-MDM population seems to correlate with the decrease of TILs at least in 99LN-BrM, it remains to be shown which consequences are emerging upon upregulation of markers related to antigenprocessing and -presentation, as commonly seen across TAMs, and especially TAMMDMs (e.g. CD74, H2-Aa, H2-Eb1; figure 21). CD74 is one of these markers related to $\mathrm{MHC}$ class II antigen presentation and an upregulation of CD74 on tumor cells of BrM was a strong positive prognostic marker and positively associated with TILs (Zeiner et al., 2018). As for the potential role of CD74 expression within the TAM compartment this effect was not investigated so far. Dichotomy of resident vs. recruited macrophages has also been observed in other neuropathological situations including an ischemic rat model (Rajan et al., 2018), or virus-induced neuroinflammation (DePaula-Silva et al., 2019). Looking beyond, yet similar to the tumor situation, disease-associated microglia (DAM) in mice and humans similarly downregulate a set of genes (e.g. Cx3cr1, P2ry12, Tmem119) related to the unique "sensome of microglia" (Hickman et al., 2013, Butovsky et al., 2014) and their homeostatic functions (Keren-Shaul et al., 2017, Mathys et al., 2017, Masuda et al., 2019; Schirmer et al., 2019). Notably however, regulation on the transcriptional level does not necessarily translate into variation on the protein level, at least shown in DAMs of some neurodegenerative models (Kraseman et al., 2017). The remarkable set of MG-specific molecular traits and phenotypic features conserved between species (Geirsdottir et al., 2019), however offers the possibility to discover certain drugs in the BrM situation, but originating from other contexts. Meta-analyses of gene expression levels across a variety of neuropathologies including ischemia, infection, inflammation, demyelination, malignancies, and neurodegeneration impressively showed similarities and differences of DAM signatures (Friedman et al., 2018). Three modules emerged as significantly important: genes belonging to proliferation, inflammation, or neurodegeneration. The later module consisted of 134 genes, and showed a stunning upregulation in almost all datasets analyzed (Friedman et al., 2018, Salamero-Boix et al., 2021). In contrast, different insults also result in diseasecontext specific MG subsets in mice and humans (Masuda et al., 2020), clearly arguing for the urgent need of a deeper characterization of cell type-specific, disease-associated molecular pattern, especially under the highly diverse brain tumor conditions. Future work additionally needs to include spatial aspects of the TME to exactly delineate BrM-associated cells from healthy ones. Particularly interesting for 
example is the MG compartment, since TAM-MG are thought to reside close to normal MG. Hence, upon removal of BrM and/or single tumor cell clusters, certain targeted approaches might help the remaining MG pool to successfully repopulate the injured area. Very recent work exploring MGs associated to Alzheimer's disease could show an in situ upregulation of certain complement members within MGs very close to $A ß$ plaques, hence arguing for locally restricted responses (Chen et al., 2020).

C) The complement system as central player in instigating inflammation in brain metastasis?

In order to identify molecular mediators of BrM-associated inflammation across various models, transcriptomic data of different TAM comparisons revealed alterations of certain members of the complement pathway. Some of them were upregulated on the protein level in H2030-BrM as well (Schulz et al., 2020), underlining their importance not only on the transcriptional level. The fundamental outcome of this innate immune pathway is the establishment of a membrane attack complex (MAC) and the release of anaphylatoxins (e.g. C3a and C5a), leading to cytolysis of target cells (MAC), and recruitment of other immune cells, respectively. Besides, it regulates a variety of complex cell-cell and cell-environment interactions and has been described as playing pivotal roles in the CNS, during development, homeostasis, and disease (e.g. Tenner et al., 2018).

Particularly interesting was the common upregulation of $C 1 q, C 3$ and $C 4 b$ in murine TAM-MGs. Although high $C 1 q$ levels belong to a conserved microglia profile across species (Geirsdottir et al., 2019), an even more enhanced expression seems to be connected to BrM-induced phenotypes in mouse, but not human BrM-MGs (Klemm et al., 2020, figure 26). Noteworthy, dramatic upregulation of C1q in MG belongs to their activation profile (Färber et al., 2009). Patient-derived TAM-MDMs however revealed higher expression levels of $C 1 Q$ members (Klemm et al., 2020, figure 26), which were also upregulated in murine TAM-MDMs. Following BrM education, it was mainly the $\mathrm{T}$ cell compartment which upregulated $\mathrm{C} 1 q$ members among TILs. Interestingly, this might be linked to variances in cellular metabolism, of especially CD8 $^{+}$T cells (Ling et al., 2018).

Another central player of the system, C3, was highly significantly upregulated in TAM-MGs of all models, but showed no apparent upregulation and partial 
downregulation in both human TAM populations associated to either B2B or L2B metastasis. This however might be because healthy human MG per se already exhibit higher levels of $C 3$ expression (Geirsdottir et al., 2019). Together the function of the major complement components are implicated to be crucial for neurological insults and its outcome. The activation most likely affects BBB integrity (Lynch et al., 2004, Jacob and Alexander, 2014), for instance, thereby enhancing inflammatory processes and mediating permeability at the BrM-periphery interface. Particularly important for that are the C3-C3aR1 and C5-C5aR1 axes, and both of the receptors were tremendously upregulated on TAM-MDMs of murine BrM models (figure 26). Moreover $\mathrm{C} 3 \mathrm{a} R 1$ expression was elevated in human MDMs of B2B and L2B metastases, as well (Klemm et al., 2020). Together the data suggest that either both or at least the C5-C5aR1 axis is critically for MDM recruitment, which consequently affects the establishment of an immuno-suppressive environment. The complement system has been extensively discussed in the context of primary brain tumors where it appears to affect maintenance of glioma stem cells (GSC), and their interaction with other cell types in the TME (reviewed in Bouwens van der Vlies et al., 2018). Since the manifold functions of complement in the context of CNS pathologies, another interesting study highlighted the C3-C3aR1 axis important for development and maintenance of leptomeningeal metastases (LM) (Boire et al., 2017). Tumor cellsecreted C3 was shown to be enriched in the CSF, and high levels were associated to poor survival rates in mouse and human. This was in part due to the upregulation of C3aR1 on the choroid plexus epithelium, which resulted in higher permeability, mediating the influx of pro-tumoral growth factors thereby fostering tumor growth. Indirectly triggered by macrophages, human LM cancer cells have been shown to upregulate specific iron-binding proteins, which supports their survival within the CSF (Chi et al., 2020). Since primary brain tumors are also frequently metastasizing to form LM, it is further tempting to speculate if the complement system may act on cellintrinsic homing mechanisms mediating this action, in a similar way like maintaining glioma stem-like cells (Bouwens van der Vlis et al., 2018). In sum, C3 seems to play a central role in BrM either in a tumor cell intrinsic and/or extrinsic fashion. The latter one might be more relevant, since it includes the interaction with other cell types of the BrM TME. Interestingly, this was shown for the crosstalk between microglia and astrocytes in the diseased brain parenchyma, which would elicit or enhance a cancer permissive environment. An integrative analysis of in vitro and in vivo experiments 
revealed a subset of neurotoxic astrocytes, which are induced via microglia-derived C1Q, TNF, and II1a. These cells termed as A1 astrocytes were present in samples of different human neuropathological conditions as well, further underlining their importance in the interplay of non-immune and immune cells upon different CNS insults (Liddelow et al., 2017). Microglia-secreted TNF however might additionally act paracine in inducing the upregulation of the "Don't-eat-me" signal CD47 within cancer cells (Betancur et al., 2017), thereby preventing phagocytosis-mediated tumor cell removal as suggested in the H2030-BrM TME (Schulz et al., 2020). This feedback-loop most likely contributes to the establishment of a pro-tumorigenic TME. Especially the interaction of astrocytes and microglia at the BrM periphery will be crucial to evaluate in more detail, since their reciprocal nature might offer the possibility to be efficiently targeted. Litvinchuk and colleagues identified STAT3 as a downstream key player of C3-C3aR1 within the astrocytes-microglia crosstalk in various neurodegenerative models, which controls a conserved network of downstream events (Litvinchuk et al., 2018). A STAT3-positive subpopulation of astrocytes has been described BrM-promoting (Priego et al., 2018). Since this crosstalk most likely appears early during BrM, rapid detection might be further exploited in order to identify molecular targets for a sustainable disruption of cancerpromoting cell-cell interaction. However technically this will be challenging, since it would imply to detect even single tumor cells prior to extravasation or shortly after.

Because expression levels of different complement members in TAMs did not change dramatically upon irradiation, the mediated effects might be independent from ionizing radiation. Hence, this offers the possibility to approach these interactions in combination with standard therapies like irradiation (e.g. WBRT), in order to interfere with both, tumor-intrinsic and -extrinsic traits fostering BrM progression. 


\section{D) WBRT influences the dynamic TME and only moderately changes TAM transcriptomes}

One major aim of this thesis was to explore the effects of WBRT on the TME in various BrM models. Quantitatively the data suggest that WBRT has the potential to influence the population size of distinct immune cells by exerting radiotoxic effects on one hand, but also enhancing the recruitment of peripheral tumor-and treatmentnaïve cells on the other hand, which is in line with previous findings (Ahn et al., 2010, Stafford et al., 2016). This effect however was model-specific and was more pronounced in xenograft models lacking the lymphoid compartment. In addition, the origin of the primary tumor plays a key role in dictating the development of its corresponding TME within the brain (Klemm et al., 2020). Hence, the tumor type itself orchestrates the effects of WBRT, as seen in the differences between the melanoma BrM (H1_DL2) and L2B metastasis (H2030) models upon IR. Interestingly, fractionated IR however similar induces constant recruitment of MDMs from the periphery in both models, although to a different extend. This is in line with a recent study from Akkari et al. (2020) showing that fractionated WBRT (5 x 2 Gy) induced an increase of the whole TAM population in glioma models, however without changing the relative contribution of each TAM type. Despite several contradictory studies regarding the effects of radiation on the BBB, it is very likely that certain radiation schemes increase the permeability of the BBB, which in addition is already disturbed in BrM (van Vulpen et al., 2002, Arvanitis et al., 2019), hence promoting the influx of cells from the periphery. This is indeed interesting since BrM-educated inflammatory cells like MDMs show higher susceptibility to undergo radiation-induced cell death, the reason radiotherapy is an interesting approach to exert immune modulatory functions within the brain TME (Sevenich, 2019). Inhibiting myeloid cell recruitment has been shown to enhance tumor responses towards radiation by eliminating pro-tumorigenic functions (Ahn et al., 2010). However, upon IR, basically two major events are likely to happen within the brain TME: 1.) induction of cell death in radio-sensitive cell types, and 2.) promotion of an enhanced recruitment due to increased BBB permeability. Both effects were seen in the H2030-BrM model upon hypo-fractionated WBRT ( $1 \times 10 \mathrm{~Gy})$. Depletion of the TAM-MDM pool was less pronounced upon $5 \times 2$ Gy at d3, whereas hypo-fractionated WBRT dramatically reduced the cell population at this time point. This can at least in part be explained by higher levels of toxicity of peripheral-derived myeloid cells. Data derived five days 
upon 1 × 10 Gy further suggest a strong re-infiltration, most likely due to an increased BBB/BTB permeability and/or changes in the secretome of tumor cells (Schulz et al., 2020). The same trend was found in the H1_DL2 model. Application of hypofractionated IR resulted in an enhanced infiltration of TAMs in a mouse model of glioma (Stafford et al., 2016). With respect to molecular changes in BrM-associated immune cells, this underlines the importance to define optimal radiation schemata including dosis and fractionation (Hellevik and Martinez-Zubiaurre, 2014, Sevenich 2019). Interestingly, single high dose fractionated RTH (1 x $10 \mathrm{~Gy})$ induced more pronounced effects on the transcriptomes of TAM-MG, TA-Monos, and TA-Granu populations, whereas the TAM-MDM population showed only a few DEGs in H2030BrM. Together, these results indicate different population turnover and/or heterogeneity in TAMs upon WBRT, a hypothesis which was proven by scRNA-Seq in H2030-BrM (Schulz et al., 2020). Pathway analyses of combined DEGs of both different treatment regimens suggested that hypo-fractionated WBRT induced proinflammatory host defense responses more efficiently compared to normal fractionation (Schulz et al., 2020).

To elucidate possible different effector functions of both major TAM populations specifically induced via IR, detailed comparative analyses between both cell types within treatment-naïve as well as treated conditions were performed. These bioinformatic comparisons underlined distinct transcriptomes segregating both cell types from each other in the BrM situation, regardless of the underlying condition. Furthermore, each condition was shaped by distinct transcription factor networks. Such ontogeny-associated gene signature panels were observed for both TAM populations in preclinical glioma models with and without fractionated IR, as well (Akkari et al., 2020). A certain overlap of similarly regulated genes in TAM-MG and TAM-MDM in either condition of BrM (H2030, not shown), or glioma (Akkari et al., 2020) reveals that in combination with standard of care, targeting total TAM populations might become a certain strategy to overcome tumor-promoting functions, and/or lifting immuno-suppressive effects related to both, TAM-MG and TAM-MDM (Akkari et al., 2020, Dumas et al., 2020, Guldner et al., 2020). In addition, both cell types were shown to limit efficacy of RTH and consequently supported recurrence of preclinical models of glioma following IR (Akkari et al., 2020). In contrast to primary brain cancers, TILs overall infiltrate at much higher levels within BrMs (Klemm et al., 2020). Hence, future studies need to focus on elucidating molecular changes of BrM- 
associated immune cells in immune-competent models to fully understand the impact of each compartment under therapeutic conditions such as WBRT.

While tumor growth rate in both xenograft models was not influenced in response to WBRT (not shown), both fractionated and hypo-fractionated WBRT induced BrM stasis in early timepoints following radiation in the 99LN model (Chae et al., 2019, Niesel et al., 2021). This in turn most likely prevented additional MDM influx at early time points in 99LN, as seen five days following $5 \times 2$ Gy. Even later (14 d), WBRTtreated 99LN-BrM did not show any differences in major myeloid immune compartments, compared to untreated tumors (Niesel et al., 2021). Contrary, within the lymphoid compartment especially $\mathrm{CD}^{+}$TILs showed reinforced infiltration into 99LN-BrM. Furthermore, the CD4:CD8 ratio was changed upon WBRT, with a more favorable environment for $\mathrm{CD}^{+}$TILs upon IR (shown here, and in Niesel et al., 2021). Taking the immuno-suppressive functions of TAM-MDMs into account, an enhanced infiltration of TILs most likely is due to an efficient, yet transient depletion of TAM-MDMs. Interestingly, total CD3 numbers were not affected in a preclinical glioma model upon WBRT when analyzed at d5 (Akkari et al., 2020). The most likely reason is a diametral population turnover between MDMs and T cells, which at one point restores the pre-WBRT situation. Despite the lack of sufficient molecular data from treated TILs of 99LN-BrM and because lymphocytes are highly sensitive towards IR (Heylmann et al., 2014), re-infiltrated TILs might show similar transcriptomic pattern as treatment-naïve TILs, since they will be treatment-naïve as well, but become BrM-educated. This is particularly important for the development of novel targeted approaches, like immune checkpoint blockade (ICB). The most efficient response to control disease progression was shown to result from a combination trial of WBRT plus ICB in the 99LN-BrM model. Nonetheless, tumor regrowth appeared after an initial stage of tumor stasis, which in part was related to the induction of compensatory immuno-suppressive mechanisms, which blunted the efficacy of radio-immunotherapy. This acquired resistance was in part mediated via re-recruitment of immuno-suppressive, treatment-naïve myeloid immune cells from the periphery (Niesel et al., 2021). Hence, approaching several targets within the TME might become relevant in the future to more efficiently prevent tumor (re-) growth in combination with WBRT as one therapeutic standard option. 


\section{E) Outlook}

In summary, within this thesis I explored the dynamical changes of the immune TME during tumor progression and upon WBRT treatment within preclinical models of $\mathrm{BrM}$, with a special focus on the two major myeloid cell populations in brain cancer: the resident microglia (TAM-MG), and monocyte-derived macrophages (TAM-MDM) infiltrating from the periphery. Interestingly, both cell types most likely differentially shape their environment and fulfill distinct functions in the crosstalk with outgrowing lesions. This is supported by spatially different pattern, as well as transcriptomic and proteomic data (Friebel et al., 2020, Klemm et al., 2020). However in the human patient scenario molecular traits underlying different outcomes are just at the beginning to be understood and need to be critically evaluated in a more differentiated way. This applies to several properties including: e.g. different primaries, different age/sex/race, or different treatment-mediated changes within the TME. Due to the complexity, preclinical approaches will be indispensable to model distinct scenarios with the overall aim to elucidate immune cell-mediated $\mathrm{BrM}$ promoting mechanisms under certain conditions. In addition to the listed modalities, spatial "omics" approaches will become more and more important in order to catch the status quo of the highly dynamic TME from a certain condition to more precisely develop novel strategies, which enhance anti-BrM immunity without exerting harmful effects on homeostatic cell functions (e.g. in microglia). The data presented in here further reveal that elucidating cellular and molecular mechanisms among distinct models has the potential to explore BrM-conservative signatures and pathways, like the proposed involvement of the complement system. Relative expression levels of certain complement members upon two distinct WBRT approaches further showed that fractionated and hypo-fractionated IR lacks the potential to influence gene expression. This implicates that the complement system might be an attractive target for future investigation in the context of adjuvant therapeutic options without becoming influenced by WBRT. Since the expression of anaphylatoxin receptors (e.g. C3AR1) was higher within the myeloid compartment, this one might become interesting to target in order to minimize or prevent the recruitment of immunesuppressive monocyte-derived cell populations (e.g. TAM-MDMs). Targeting TAMs, either MG+MDM, or MDMs alone has been promising in several preclinical studies of 
brain cancer, with and without further therapeutic intervention (Pyonteck et al., 2013, Quail et al., 2016, Sevenich 2018, Akkari et al., 2020, Klemm et al., in revision).

To expand the focus beyond immune cells within the TME, other brain-resident cells (e.g. astrocytes, neurons) or endothelial cells of the BrM-associated vasculature require additional examination to fully understand their impact on BrM biology. Because the vasculature has been proposed to be one key player in regulating BrM infiltration, examining their cellular composition upon BrM on the molecular level will furthermore shape our knowledge and will in combination with targeted approaches (e.g. complement) open new avenues to tackle this deadly disease. One recent study for example finds pericytes, which are associated to the vasculature, as contributing to immune cell infiltration into the parenchyma since their absence facilitates neuroinflammation (Török et al., 2021). Moreover, pericytes have been shown to promote BrM formation (Molnar et al., 2020), but their roles related to immune cell trafficking in the BrM situation remain to be elucidated.

In summary, further in vivo analyzes will be crucial to understand the BrM-promoting environment. State-of-the-art techniques will open unprecedented insight into molecular traits of BrM-associated inflammation. Nevertheless, while targeting certain cell types or pathways it will be challenging to minimize side effects or to prevent interference with homeostatic functions of other resident cell types (e.g. MG), which are not associated to any lesions in brains affected by metastases. 


\section{References}

Abbott, N. J. (2013). Blood-brain barrier structure and function and the challenges for CNS drug delivery. J Inherit Metab Dis. 36: 437 - 449.

Adler, D., Murdoch, D. and others (2020). rgl: 3D Visualization Using OpenGL. R package version 0.100.54. https://CRAN.R-project.org/package=rgl.

Ahn, G., Tseng, D., Liao, C.-H., Dorie, M. J., Czechowicz, A. and Brown, J. M. (2010). Inhibition of Mac-1 (CD11b/CD18) enhances tumor response to radiation by reducing myeloid cell recruitment. Proc Natl Acad Sci. 107(18): 8363 - 8368.

Ajami, B., Samusik, N., Wieghofer, P., Ho, P. P., Crotti, A., Bjornson, Z., Prinz, M., Fantl, W. J., Nolan, G. P. and Steinman, L. (2018). Single-cell mass cytometry reveals distinct populations of brain myeloid cells in mouse neuroinflammation and neurodegeneration models. Nat Neurosci. 21: 541-551.

Akkari, L., Bowman, R. L., Tessier, J., Klemm, F., Handgraaf, S. M., de Groot, M., Quail, D. F., Tillard, L., Gadiot, J., Huse, J. T., Brandsma, D., Westerga, J., Watts, C. and Joyce, J. A. (2020). Dynamic changes in glioma macrophage populations after radiotherapy reveal CSF-1R inhibition as a strategy to overcome resistance. Sci Transl Med. 12: eaaw7843.

Anders, S., Pyl, P. T. and Huber, W. (2015) HTSeq - a python framework to work with high-throughput sequencing data. Bioinformatics. 31(2): 166 - 169.

Arvanitis C. D., Ferraro, G. B. and Jain, R. K. (2019). The blood-brain barrier and bloodtumour barrier in brain tumours and metastasis. Nat Rev Cancer. 20: 26-41.

Aspelund, A., Antila, S., Proulx, S. T., Karlsen, T. V., Karaman, S., Detmer, M., Wiig, H. and Alitalo, K. (2015). A dural lymphatic vascular systesm that drains brain interstitial fluid and macromolecules. J Exp Med. 212(7): $991-999$.

Babicki, S., Arndt, D., Marcu, A., Liang, Y., Grant, J. R., Maciejewski, A. and Wishart, D. S. (2016). Heatmapper: web-enabled heat mapping for all. Nucleic Acids Res. doi: 10.1093/nar/gkw419.

Berghoff, A. S., Ricken, G., Widhalm, G., Rajky, O., Dieckmann, K., Birner, P., Bartsch, R., Höller, C. and Preusser, M. (2014). Tumour-infiltrating lymphocytes and expression of programmed death ligand 1 (PD-L1) in melanoma brain metastases. Histopathology. 66(2): $289-299$.

Berghoff, A. S., Fuchs, E., Ricken, G., Mlecnik, B., Bindea, G., Spanberger, T., Hackl, M., Widhalm, G., Dieckmann, K., Prayer, D., Bilocq, A., Heinzl., H., Zielinski, C., Bartsch, R., Birner, P., Galon, J. and Preusser, M. (2016). Density of tumor-infiltrating lymphocytes correlates with extent of brain edema and overall survival time in patients with brain metastases. Oncolmmunology. 5(1): e1057388, DOI: 10.1080/2162402X.2015.1057388.

Betancur, P. A., Abraham, B. J., Yiu, Y. Y., Willingham, S. B., Khameneh, F., Zarnegar, M., Kuo, A. H., McKenna, K., Kojima, Y., Leeper, N. J., Ho, P., Gip, P., Swigut, T., Sherwood, R. I., Clarke, M. F., Somlo, G., Young, R. A. and Weissman, I. L. (2017). A CD47-associated super-enhancer links pro-inflammatory signalling to CD47 upregulation in breast cancer. Nat Commun. 14802.

Boire, A., Zou, Y., Shieh, J., Macalinao, D. G., Pentsova, E. and Massague, J. (2017). Complement Component 3 Adapts the Cerebrospinal Fluid for Leptomeningeal Metastasis. Cell. 168: $1101-1113$.

Bos, P. D., Zhang, X. H.-F., Nadal, C., Shu, W., Gomis, R. R., Nguyen, D. X., Minn, A. J., van de Vijver, M. J., Gerald, W. L., Foekens, J. A. and Massague, J. (2009). Genes that mediate breast cancer metastasis to the brain. Nature. 458: $1005-1009$.

Bouwens van der Vlies, T. A. M., Kros, J. M., Mustafa, D. A. M., van Wijck, R. T. A., Ackermans, L., van Hagen, P. M. and van der Spek, P. J. (2018). The complement system in glioblastoma multiforme. Acta Neuropathol Commun. 6: 91. 
Bowman, R. L., Klemm, F., Akkari, L., Pyonteck, S. M., Sevenich, L., Quail, D. F., Dhara, S., Simpson, K., Gardner, E. E., lacobuzio-Donahue, C. A., Brennan, C. W., Tabar, V., Gutin, P. H. and Joyce, J. A. (2016). Macrophage Ontogeny Underlies Differences in Tumor-Specific Education in Brain Malignancies. Cell Rep. 17(9): 2445 - 2459.

Böttcher, C. and Priller, J. (2016). Myeloid cell-based therapies in neurological disorders: How far have we come?. Biochim et Biophys Acta. 1862: 323 - 328.

Brastianos, P. K., Carter, S. L., Santagata, S., Cahill, D. P., Taylor-Weiner, A., Jones, R. T., Van Allen, E. M., Lawrence, M. S., Horowitz, P. M., Cibulskis, K., Ligon, K. L., Tabernero, J., Seoane, J., Martinez-Saez, E., Curry, W. T., Dunn, I. F., Ha Paek, S., Park, S. H., McKenna, A., Chevalier, A., Rosenberg, M., Barker II., F. G., Gill., C. M., Van Hummelen, P., Thorner, A. R., Johnson, B. E., Hoang, M. P., Choueiri, T. K., Signoretti, S., Sougnez, C., Rabin, M. S., Lin, N. U., Winer, E. P., Stemmer-Rachamimov, A., Meyerson, M., Garraway, L., Gabriel, S., Lander, E. S., Beroukhim, R., Batchelor, T. T., Baselga, J., Louis, D. N., Getz, G. and Hahn, W. C. (2015). Genomic Characterization of Brain Metastases Reveals Branched Evolution and Potential Therapeutic Targets. Cancer Discov. 5(11): 1164 - 1177.

Brendel, C., Goebel, B., Daniela, A., Brugman, M., Kneissl, S., Schwäble, J., Kaufmann, K. B., Müller-Kuller, U., Kunkel, H., Chen-Wichmann, L., Abel, T., Serve, H., Bystrykh, L., Buchholz, C. J. and Grez, M. (2014). CD133-targeted Gene Transfer Into Long-term Repopulating Hematopoietic Stem Cells. Mol Ther. 23(1): 63 - 70.

Brown, G. C. and Neher, J. J. (2010). Inflammatory Neurodegeneration and Mechanisms of Microglial Killing of Neurons. Mol. Neurobiol. 41: $242-247$.

Butovsky, O., Jedrychowski, M. P., Moore, C. S., Cialic, R., Lanser, A. J., Gabriely, G., Koeglsperger, T., Dake, B., Wu, P. M., Doykan, C. E., Fanek, Z., Liu, L., Chen, Z., Rothstein, J. D., Ransohoff, R. M., Gygi, S. P., Antel, J. P. and Weiner, H. L. (2014). Identification of a unique TGF-beta-dependent molecular and functional signature in microglia. Nat Neurosci 17: 131-143.

Chae, W. H., Niesel, K., Schulz, M., Klemm, F., Joyce, J. A., Prümmer,M.,

Brill, B., Bergs, J., Rödel, F., Pilatus, U. and Sevenich, L. (2019). Evaluating Magnetic Resonance Spectroscopy as a Tool for Monitoring Therapeutic Response of Whole Brain Radiotherapy in a Mouse Model for Breast-to-Brain Metastasis. Front Oncol. 9: 1324. doi: 10.3389/fonc.2019.01324.

Chen, Q., Boire, A., Jin, X., Valiente, M., Er, E. E., Lopez-Soto, A., Jacob, L.

S., Patwa, R., Shah, H., Xu, K., Cross, J. R. and Massagué, J. (2016). Carcinomaastrocyte gap junctions promote brain metastasis by cGAMP transfer. Nature. 533: $493-497$.

Chen, Z., Feng, X., Herting, C. J., Garcia, V. A., Nie, K., Pong, W. W., Rasmussen, R., Dwivedi, B., Seby, S., Wolf, S. A., Gutmann, D. H. and Hambardzumyan, D. (2017). Cellular and molecular identity of tumor-associated macrophages in glioblastoma. Cancer Res. 77(9): 2266 - 2278.

Chen, W.-T., Lu, A., Craessaerts, K., Pavie, B., Frigerio, C. S., Corthout, N., Q., X.,

Lalakova, J., Kühnemund, M., Voytyuk, I., Wolfs, L., Mancuso, R., Salta, E., Balusu, S., Snellinx, A., Munck, S., Jurek, A., Fernandez Navarro, J., Saido, T. C., Huitinga, I., Lundeberg, J., Fiers, M. and De Strooper, B. (2020). Spatial Transcriptomics and In Situ Sequencing to Study Alzheimer's Disease. Cell. 182: 976 - 991.

Chi, Y., Remsik, J., Kiseliovas, V., Derderian, C., Sener, U., Alghader, M., Saadeh, F., Nikishina, K., Bale, T., lacobuzio-Donahue, C., Thomas, T., Pe'er, D., Mazutis, L. and Boire, A. (2020). Cancer cells deploy lipocalin-2 to collect limiting iron in leptomeningeal metastasis. Science. 369: $276-282$.

Chuang, H. N., van Rossum, D., Sieger, D., Siam, L., Klemm, F., Bleckmann, A., Bayerlova, M., Farhat, K., Scheffel, J., Schulz, M., Dehghani, F., Stadelmann, C., Hanisch, U.-K., Binder, C. and Pukrop. T. (2013). Carcinoma cells misuse the host tissue damage response to invade the brain. Glia. 61(8): 1331 - 1346.

Colombo, E. and Farina, C. (2016) Astrocytes: Key Regulators of Neuroinflammation. Trends Immunol. 37(9): 608-620. 
Darmanis, S., Sloan, S. A., Croote, D., Mignardi, M., Chernikova, S., Samghababi, P., Zhang, Y., Neff, N., Kowarsky, M., Caneda, C., Li, G., Chang, S. D., Connolly, I. D., Li, Y., Barres, B. A., Gephart, M. H. and Quake, S. R. (2017). Single-Cell RNA-Seq Analysis of Infiltrating Neoplastic Cells at the Migrating Front of Human Glioblastoma. Cell Rep. 21(5): 1399 - 1410.

Davoodzhadeh Gholami, M., kardar, G., Saeedi, Y., Heydari, S., Garssen, J. and Falak, R. (2017). Exhaustion of T lymphocytes in the tumor microenvironment: significance and effective mechanisms. Cell Immunol. 322: 1 - 14.

DePaula-Silva, A. B., Gorbea, C., Doty, D. J., Libbey, J. E., Sanchez, J. M. S., Hanak, T. J., Cazalla, D. and Fujinami, R. S. (2019). Differential transcriptional profiles identify microglial- and macrophage-specific gene markers expressed during virus-induced neuroinflammation. J Neuroinflammation. 16: 152.

Doron, H., Amer, M., Ershaid, N., Blazquez, R., Shani, O., Lahav, T, G., Cohen, N., Adler, O., Hakim, Z., Pozzi, S., Scomparin, A., Cohen, J., Yassin, M., Monteran, K., Grossman, R., Tsarfaty, G., Luxenburg, C., Satchi-Fainaro, R., Pukrop, T. and Erez, N. (2019). Inflammatory Actiation of Astrocytes Facilitates Melanoma Brain Tropism via the CXCL10-CXCR3 Signaling Axis. Cell Rep. 28: 1785 - 1798.

Dragulescu, A. and Arendt, C. (2020). xlsx: Read, Write, Format Excel 2007 and Excel 97/2000/XP/2003 Files. R package version 0.6.4.2. https://CRAN.Rproject.org/package $=x \mathrm{xls}$.

Dumas, A. A., Pomella, N., Rosser, G., Guglielmi, L., Vinel, C., Millner, T. O., Rees, J., Aley, N., Sheer, D., Wei, J., Marisetty, A., Heimberger, A. B., Bowman, R. L., Brandner, S., Joyce, J. A., Marino, S. (2020). Microglia promote glioblastoma via mTOR-mediated immunosuppression of the tumour microenvironment. EMBO J. e103790. Doi: 10.15252/embj.2019103790.

Durinck, S., Spellman, P. T., Birney, E. and Huber, W. (2009). Mapping identifiers for the integration of genomic datasets with the R/Bioconductor package biomaRt. Nat Protoc. 4(8): $1184-1191$.

Färber, K., Cheung, G., Mitchell, D., Wallis, R., Weihe, E., Schwaeble, W. and Kettenmann, H. (2009). C1q, the Recognition Subcomponent of the Classical Pathway of Complement, Drives Microglial Activation. J Neurosci Res. 87: 644 - 652.

Flemming, A., Huang, Q.-Q., Jin, J.-P., Jumaa, H. and Herzog, S. (2015). A Conditional Knockout Mouse Model Reveals That Calponin-3 Is Dispensable for Early B Cell Development. PLoS One. 10(6): e0128385.

Fokas, E., Steinbach, J. P. and Rödel, C. (2013). Biology of brain metastases and novel targeted therapies: Time to translate the research. Biochim et Biophys Acta. 1835(1): 61-75.

Friebel, E., Kapolou, K., Unger, S., Nunez, N. G., Utz, S., Rushing, E. J., Regli, L., Weller, M., Greter, M., Tugues, S., Neidert, M. C. and Becher, B. (2020). Single-Cell Mapping of Human Brain Cancer Reveals Tumor-Specific Instruction of Tissue-Invading Leukocytes. Cell. 181: 1-17.

Friedman, B. A., Srinivasan, K., Ayalon, G., Meilandt, W. J., Lin, H., Huntley, M. A., Cao, Y., Lee, S.-H., Haddick, P. C. G., Ngu, H., Modrusan, Z., Larson, J. L., Kaminker, J. S., van der Brug, M. P. and Hansen, D. V. (2018). Diverse Brain Myeloid Expression Profiles Reveal Distinct Microglial Activation States and Aspects of Alzheimer's Disease Not Evident in Mouse Models. Cell Rep. 22: $832-847$.

Garcia, A. D. R., Doan, N. B., Imura, T., Bush, T. G. and Sofroniew, M. V. (2004). GFAP-expressing progenitors are the principal source of constitutive neurogenesis in adult mouse forebrain. Nat Neurosci. 7(11): 1233 - 1241.

Gavrilovic, I. T. and Posner, J. B. (2005). Brain metastases: epidemiology and pathophysiology. J Neuro-Oncol. 75: 5 - 14.

Geirsdottir, L., David, E., Keren-Shaul, H., Weiner, A., Bohlen, S. C., Neuber, J., Balic, A., Giladi, A., Sheban, F., Dutertre, C.-A., Pfeifle, C., Peri, F., Raffo-Romero, A., Vizioli, J., Matiasek, K., Scheiwe, C., Meckel, C., Mätz-Rensing, K., van der Meer, F., Thormodsson, F. R., Stadelmann, C., Zilkha, N., Kimchi, T., Ginhoux, F., Ulitsky, 
I., Erny, D., Amit, I. and Prinz, M. (2019). Cross-Species Single-Cell Analysis Reveals Divergence of the Primate Microglia Program. Cell. 179: 1609 - 1622.

Ginhoux, F., Greter, M., Leboeuf, M., Nandi, S., See, P., Gokhan, S., Mehler,

M. F., Conway, S. J., Ng, L. G., Stanley, E. R., Samokhvalov, I. M. and Merad, M. (2010). Fate Mapping Analysis Reveals That Adult Microglia Derive from Primitive Macrophages. Science. 330: $841-845$.

Goldmann, T., Wieghofer, P., Jordao, M. J. C., Prutek, F., Hagemeyer, N., Frenzel, K., Amann, L., Staszewski, O., Kierdorf, K., Krueger, M., Locatelli, G., Hochgerner, H., Zeiser, R., Epelman, S., Geissmann, F., Priller, J., Rossi, F. M. V., Bechmann, I., Kerschensteiner, M., Linnarsson, S., Jung, S. and Prinz, M. (2016). Origin, fate and dynamics of macrophages at central nervous system interfaces. Nat Immunol.17(7): $797-805$.

Grün, D., Muraro, M. J., Boisset, J. C., Wiebrands, K., Lyubimova, A., Dharmadhikari, G., van den Born, M., van Es, J., Jansen, E., Clevers, H., de Koning, E. J. P. and van Oudenaarden, A. (2016). De Novo Prediction of Stem Cell Identity using Single-Cell Transcriptome Data. Cell Stem Cell. 19: 266 - 277.

Guldner, I. H., Wang, Q., Yang, L., Golomb, S. M., Zhao, Z., Lopez, J. A., Brunory, A., Howe, E. N., Zhang, Y., Palakurthi, B., Barron, M., Gao, H., Xuei, X., Liu, Y., Li, J., Chen, D. Z., Landreth, G. E. and Zhang, S. (2020). CNS-Native Myeloid Cells Drive Immune Suppression in the Brain Metastatic Niche through Cxcl10. Cell. 183: 1-15.

Gutmann, D. H. and Kettenmann, H. (2019). Microglia/Brain Macrophages as Central Drivers of Brain Tumor Pathobiology. Neuron. 104: $442-449$.

Han, H., Cho, J.-W., Lee, S., Yun, A., Kim, H., Bae, D., Yang, S., Kim, C. Y., Lee, M., Kim, E., Lee, S., Kang, B., Jeong, D., Kim, Y., Jeon, H.-N., Jung, H., Nam, S., Chung, M., Kim, J.-H. and Lee, I. (2017). TRRUST v2: an expanded reference database of human and mouse transcriptional regulatory interactions. Nuc Acid Res. 46: D380 D386.

Hanahan, D. and Weinberg, R. A. (2000). The Hallmarks of Cancer. Cell. 100: 57 -70.

Harter, P. N., Bernatz, S., Scholz, A., Zeiner, P. S., Zinke, J., Kiyose, M., Blasel, S., Beschorner, R., Senft, C., Bender, B., Ronellenfitsch, M. W., Wikman, H., Glatzel, M., Meinhardt, M., Juratli, T. A., Steinbach, J. P., Plate, K. H., Wischhusen, J., Weide, B. and Mittelbronn, M. (2015). Distribution and prognostic relevance of tumor-infiltrating lymphocytes (TILs) and PD-1/PD-L1 immune checkpoints in human brain metastasis. Oncotarget. 6(38): $40836-40849$.

Hellevik, T. and Martinez-Zubiaurre, I. M. (2014). Radiotherapy and the tumor stroma: the importance of dose and fractionation. Front Oncol. 4(1): 1.

Herisson, F., Frodermann, V., Courties, G., Rohde, D., Sun, Y., Vandoorne, K., Wojtkiewicz, G. R., Santos Masson, G., Vinegoni, C., Kim, J., Kim, D.-E., Weissleder, R., Swirski, F. K., Moskowitz, M. A. and Nahrendorf, M. (2018). Direct vascular channels connect skull bone marrow and the brain surface enabling myeloid cell migration. Nat Neurosci. 21: $1209-1217$.

Heylmann, D., Rödel, F., Kindler, T. and Kaina, B. (2014). Radiation sensitivity of human and murine peripheral blood lymphocytes, stem and progenitor cells. Biochim Biophys Acta. 1846: 121 - 129.

Hickman, S. E., Kingery, N. D., Ohsumi, T. K. Borowsky, M. L., Wang, L. C., Means, T. K. and El Khoury, J. (2013). The Microglial Sensome Revealed by Direct RNA Sequencing. Nat Neurosci. 16: $1896-1905$.

Hide, T., Komohara, Y., Miyasato, Y., Nakamura, H., Makino, K., Takeya, M., Kuratsu, J.-i., Mukasa, A. and Yano, S. (2018). Oligodendrocyte Progenitor Cells and Macrophages/Microglia Produce Glioma Stem Cell Niches at the Tumor Border. EbioMedicine. 30: 94 -104.

Hofmarcher, T., Bradvik, G., Svedman, C., Lindgren, P., Jönsson, B. and Wilking, N. (2019). Comparator Report on Cancer in Europe 2019 - Disease Burden, Costs and Access to Medicines. IHE Report 2019: 7. IHE: Lund, Sweden. 
Huang, Y., Hoffman, C., Rajappa, P. Kim, J.-H., Hu, W., Huse, J., Tang, Z., Li,

X., Weksler, B., Bromberg, J., Lyden, D. C. and Greenfield, J. P. (2014). Oligodendrocyte progenitor cells promote neovascularization in glioma by disrupting the blood-brain barrier. Cancer Res. 74(4): 1011 - 1021.

Huysamen, C., Willment, J. A., Dennehy, K. M. and Brown, G. D. (2008). CLEC9A Is a Novel Activation C-type Lectin-like Receptor Expressed on $\mathrm{BDCA}^{+}$Dendritic Cells and a Subset of Monocytes. J Biol Chem. 283(24): 16693 - 16701.

Jacob, A. and Alexander, J. J. (2014). Complement and blood-brain barrier integrity. Mol Immunol. 61: 149 - 152.

Jäkel, S., Agirre, E., Mendanha Falcao, A., van Bruggen, D., Lee, K. W., Knuesel, I., Malhotra, D., ffrench-Constant, C., Williams, A. and Castelo-Branco, G. (2019). Altered human oligodendrocyte heterogeneity in multiple sclerosis. Nature. 566: $543-547$.

Jordao, M. J. C., Sankowski, R., Brendecke, S. M., Sagar, Locatelli, G., Tai, Y. H., Tay, T. L., Schramm, E., Armbruster, S., Hagemeyer, N., Gross, O., Mai, D., Cicek, O., Falk, T., Kerschensteiner, M., Grün, D. and Prinz, M. (2019). Single-cell profiling identifies myeloid cell subsets with distinct fates during neuroinflammation. Science. 363: eaat7554.

Jung, S., Aliberti, J., Graemmel, P., Sunshine, M. J., Kreutzberg, G. W., Sher, A. and Littman, D. R. (2000). Analysis of Fractalkine Receptor CX3CR1 Function by Targeted Deletion and Green Fluorescent Protein Reporter Gene Insertion. Mol Cell Biol. 20(11): 4106 - 4114.

Keren-Shaul., H., Spinrad, A., Weiner, A., Matcovitch-Natan, O., Dvir-Szternfeld, R., Ulland, T. K., David, E., Baruch, K., Lara-Astaiso, D., Toth, B., Itzkovitz, S., Colonna, M., Schwartz, M. and Amit, I. (2017). A Unique Microglia Type Associated with Restricting Development of Alzheimer's Disease. Cell. 169: 1276 - 1290.

Khakh, B. S. and Sofroniew, M. V. (2015). Diversity of astrocyte functions and phenotypes in neural circuits. Nature Neuroscience. 18 (7): 942 - 952.

Kienast, Y., von Baumgarten, L., Fuhrmann, M., Klinkert, W. E. F., Goldbrunner, R., Herms, J. and Winkler, F. (2010). Real-time imaging reveals the single steps of brain metastasis formation. Nat Med. 16(1): 116- 122.

Kim, S.-J., Kim, J.-S., Park, E. S., Lee, J.-S., Lin, Q., Langley, R. R., Maya,

M., He, J., Kim, S.-W., Weihua, Z., Balasubramanian, K., Fan, D., Mills, G. B., Hung, M.-C. and Fidler, I. J. (2011). Astrocytes Upregulate Survival Genes in Tumor Cells and Induce Protection from Chemotherapy. Neoplasia. 13(3): $286-298$.

Klemm, F., Maas, R. R., Bowman, R. L., Kornete, M., Soukup, K., Nassiri, S., Brouland, J.-P., lacobuzio-Donahue, C. A., Brennan, C., Tabar, V., Gutin, P. H., Daniel, R. T., Hegi, M. E. and Joyce, J. A. (2020). Interrogation of the microenvironmental Landscape in Brain Tumors Reveals Disease-Specific Alterations of Immune Cells. Cell. 181: 1-18.

Klemm, F., Moeckl* A., Salamero-Boix*, A., Alekseeva, T., Schaeffer, A., Schulz, M., Niesel, K., Maas, R. R., Groth, M., Elie, B. T., Bowman, R. L., Hegi, M. E., Daniel, R. T., Zeiner, P. S., Zinke, J., Harter, P. N., Plate, K. H., Joyce, J. A. and Sevenich, L. (2021). Compensatory CSF-2-driven macrophage activation promotes adaptive resistance to CSF1R inhibition in breast-to-brain metastasis. Nat Cancer. accepted.

Kolde, R. (2019). pheatmap: Pretty Heatmaps. https://CRAN.R-project.org/package=pheatmap.

Koo, C. Z., Harrison, N., Noy, P. J., Szyroka, J., Matthews, A. L., Hsia, H.-E., Müller, S. A., Tüshaus, J., Goulding, J., Willis, K., Apicella, C. Cragoe, B., Davis, E., Keles, M., Malinova, A., McFarlane, T. A., Morrison, P. R., Nguyen, H. T. H., Sykes, M., Ahmed, H., Di Maio, A., Seipold, L., Saftig, P., Cull, E., Pliotas, C., Rubinstein, E., Poulter, N. S., Briddon, S. J., Holliday, N. D., Lichtenthaler, S. F. and Tomlinson, M. G. (2020). The tetraspanin Tspan15 is an essential subunit of an ADAM10 scissor complex. J Biol Chem. 295(36): 12822 - 12839. 
Korin, B., Ben-Shaanan, T. L., Schiller, M., Dubovik, T., Azulay-Debby, H., Boshnak, N. T., Koren, T. and Rolls, A. (2017). High-dimensional, single-cell characterization of the brain's immune compartment. Nat Neurosci. 20: $1300-1309$.

Kraseman, S., Madore, C., Cialic, R., Baufeld, C., Calcagno, N., El Fatimy, R., Beckers, L., O'Loughlin, E., Xu, Y., Fanek, Z., Greco, D. J., Smith, S. T., Tweet, G., Humulock, Z., Zrzavy, T., Conde-Sanroman, P., Gacias, M., Weng, Z., Chen, H., Tjon, E., Mazaheri, F., Hartmann, K., Madi, A., Ulrich, J. D., Glatzel, M., Worthmann, A., Heeren, J., Budnik, B., Lemere, C., Ikezu, T., Heppner, F. L., Litvak, V., Holtzman, D. M., Lassmann, H., Weiner, H. L., Ochando, J., Haass, C. and Butovsky, O. (2017). The TREM2-APOE Pathway Drives the Transcriptional Phenotype of Dysfunctional Microglia in Neurodegenerative Diseases. Immunity. 47: 566 - 581.

Larsson J (2020). eulerr: Area-Proportional Euler and Venn Diagrams with Ellipses. https://cran.r-project.org/package=eulerr.

Lehrer, E. J., McGee, H. M., Sheehan, J. P. and Trifiletti, D. M. (2020). Integration of immune-oncology with stereotactic radiosurgery in the management of brain metastases. J Neuro-Oncol. doi: 10.1007/s11060-020-03427-6.

Liddelow, S. A. and Barres, B. A. (2017). Reactive Astrocytes: Production, Function, and Therapeutic Potential. Immunity. 46: 957 - 967.

Liddelow, S. A., Guttenplan, K. A., Clarke, L. E., Bennett, F. C., Bohlen, C. J., Schirmer, L., Bennett, M. L., Münch, A. E., Chung, W.-S., Peterson, T. C., Wilton, D. K., Frouin, A., Napier, B. A., Panicker, N., Kumar, M., Buckwalter, M. S., Rowitch, D. H., Dawson, V. L., Dawson, T. M., Stevens, B. and Barres, B. (2017). Neurotoxic reactive astrocytes are induced by activated microglia. Nature. 541: $481-487$.

Lin, Q., Balasubramanian, K., Fan, D., Kim, S.-J., Guo, L., Wang, H., Bar-Eli, M., Aldape, K. D. and Fidler, I. J. (2010). Reactive Astrocytes Protect Melanoma Cells from Chemotherapy by Sequestering Intracellular Calcium through Gap Junction Communication Channels. Neoplasia. 12(9): 748 - 754.

Lin, X. and DeAngelis, L. M. (2015). Treatment of Brain Metastases. J Clin Oncol. 33(30): $3475-3484$.

Ling, G. S., Crawford, G., Buang, N., Bartok, I., Tian, K., Thielens, N. M., Bally, I., Harker, J. A., Ashton-Rickardt, P. G., Rutschmann, S., Strid, J. and Botto, M. (2018). C1q restrains autoimmunity and viral infection by regulating CD8+ $\mathrm{T}$ cell metabolism. Science. 360: $558-563$.

Litvinchuk, A., Wan, Y.-W., Swartzlander, D. B., Chen, F., Cole, A., Propson, N. E., Wang, Q., Zhang, B., Liu, Z. and Zheng, H. (2018). Complement C3aR Inactivation Attenuates Tau Pathology and Reverses an Immune Network Deregulated in Tauopathy Models and Alzheimer's Disease. Neuron. 100: 1337 - 1353.

Lorger, M. and Feldling-Habermann, B. (2010). Capturing changes in the brain microenvironment during initial steps of breast cancer brain metastasis. Am J Pathol. 176(6): 2958 - 2971.

Louveau, A., Smirnov, I., Keyes, T. J., Eccles, J. D., Rouhani, S. J., Peske, J. D., Derecki, N. C., Castle, D., Mandell, J. W., Lee, K. S., Harris, T. H. and Kipnis, J. (2015). Structural and functional features of central nervous system lymphatic vessels. Nature. 523: 337 - 341.

Louveau, A., Herz, J., Alme, M. N., Salvador, A. F., Dong, M. Q., Viar, K. E., Herod, S. G., Knopp, J., Setliff, J. C., Lupi, A. L., Da Mesquita, S., Frost, E. L., Gaultier, A., Harris, T. H., Cao, R., Hu, S., Lukens, J. R., Smirnov, I., Overall, C. C., Oliver, G. and Kipnis, J. (2018). CNS lymphatic drainage and neuroinflammation are regulated by meningeal lymphatic vasculature. Nat Neurosci. 21: 1380 - 1391.

Love, M. I., Huber, W. and Anders, S. (2014). Moderated estimation of fold change and dispersion for RNA-Seq data with DESeq2. Genome Biol. 15: 550.

Lowe, S. S., Danielson, B., Beaumont, C.,Watanabe, S. M., Baracos, V. E. and Courneya, K. S. (2014). Association Between Objectively Measured Physical Activity and Quality of Life in Cancer Patients With Brain Metastases. J Pain Symptom Manag. 48(3): $322-332$. 
Lynch, N. J., Willis, C. L., Nolan, C. C., Roscher, S., Fowler, M. J., Weihe, E., Ray, D. E. and Schwaeble W. J. (2004). Microglial activation and increased synthesis of complement component C1q precedes blood-brain barrier dysfunction in rats. Mol Immunol. 40: $709-716$.

Madisen, L., Zwingman, T. A., Sunkin, S. M., Wook Oh., S., Zariwala, H. A., Gu, H., Ng, L. L., Palmiter, R. D., Hawrylycz, M. J., Jones, A. R., Lein, E. S. and Zeng, H. (2010). A robust and high-throughput Cre reporting and characterization system for the whole mouse brain. Nat Neurosci. 13: 133 - 144.

Mantovani, A., Marchesi, F., Malesci, A., Laghi, L. and Allavena, P. (2017).

Tumour-associated macrophages as treatment targets in oncology. Nat Rev Clin Oncol. 14: $399-416$.

Margolin, K., Ernstoff, M. S., Hamid, O., Lawrence, D., McDermott, D., Puzanov, I., Wolchok, J. D., Clark, J. I., Sznol, M., Logan, T. F., Richards, J., Michener, T., Balogh, A., Heller, K. N. and Hodi, F. S. (2012). Ipilimumab in patinets with melanoma and brain metastases: an open-label, phase 2 trial. Lancet Oncol. 13: $459-465$.

Massague, J. and Obenauf, A. C. (2016). Metastatic colonization by circulating tumour cells. Nature. 529: 298-306.

Masuda, T., Sankowski, R., Staszewski, O., Böttcher, C., Amann, L., Sagar, Scheiwe, C., Nessler, S., Kunz, P., van Loo, G., Coenen, V. A., Reinacher, P. C., Michel, A., Sure, U., Gold, R., Grün, D., Priller, J., Stadelmann, C. and Prinz, M. (2019). Spatial and temporal heterogeneity of mouse and human microglia at single-cell resolution. Nature. 566: $388-392$.

Masuda, T., Sankowski, R., Staszewski, O. and Prinz, M. (2020). Microglia Heterogeneity in the Single-Cell Era. Cell Rep. 30(5): $1271-1281$.

Mathys, H., Adaikkan, C., Gao, F., Young, J. Z., Manet, E., Hemberg, M., De Jager, P. L., Ransohoff, R. M., Regev, A. and Tsai, L.-H. (2017). Temporal Tracking of Microglia Activation in Neurodegeneration at Single-Cell Resolution. Cell Rep. 21: $366-380$.

McTyre, E., Scott, J. and Chinnaiyan, P. (2013). Whole brain radiotherapy for brain metastasis. Surg Neurol Int. 4: 236 - 244.

Molnar, K., Meszaros, A., Fazakas, C., Kozma, M., Györi, F., Reisz, Z., Tiszlavicz, L., Farkas, A. E., Nyul-Toth, A., Hasko, J., Krizbai, I. A. and Wilhelm, I. (2020). Pericytesecreted IGF2 promotes breast cancer brain metastasis. Mol Oncol. 14(9): 2040 2057.

Moyers, J. T., Chong, E. G., Peng, J., Tsai, H. H. C., Sufficool, D., Shavlik, D. and Nagaraj, G. (2021). Real world outcomes of combination and timing of immunotherapy with radiotherapy for melanoma brain metastases. Cancer Med. doi:10.1002/cam4.3716. online ahead of print.

Mrdjen, D., Pavlovic, A., Hartmann, F. J., Schreiner, B., Utz, S. G., Leung, B. P., Lelios, I., Heppner, F. L., Kipnis, J., Merkler, D., Greter, M. and Becher, B. (2018). HighDimensional Single-Cell Mapping of Central Nervous System Immune Cells Reveals Distinct Myeloid Subsets in Health, Aging, and Disease. Immunity. 48: 380 - 395.

Muller, A., Brandenburg, S., Turkowski, K., Muller, S. and Vajkoczy, P. (2015). Resident microglia, and not peripheral macrophages, are the main source of brain tumor mononuclear cells. Int J Cancer. 137(2): 278 - 88.

Muraro, M. J., Dharmadhikari, G., Grün, D., Groen, N., Dielen, T., Jansen, E., van Gurp, L., Engelse, M. A., Carlotti, F., de Koning, E. J. and van Oudenaarden, A. (2016). A Single-Cell Transcriptome Atlas of the Human Pancreas. Cell Syst. 3: 385394 e383.

Murray, P.J. (2017). Macrophage Polarization. Annu Rev Physiol. 79: 541 - 566.

Nayak, L., Quant Lee, E. and Wen, P. Y. (2012). Epidemiology of Brain Metastases. Curr Oncol Rep. 14: 48-54. 
Neman, J., Termini, J., Wilczynski, S., Vaidehi, N., Choy, C., Kowolik, C. M., Li, H., Hambrecht, A. C., Roberts, E. and Jandial, R. (2014). Human breast cancer metastases to the brain display GABAergic properties in the neural niche. Proc Natl Acad Sci. 111(3): $984-989$.

Neuwirth, E. (2014). RColorBrewer: ColorBrewer Palettes. https://cran.r-project.org/web/packages/RColorBrewer/index.html.

Nguyen, D. X., Chiang, A. C., Zhang, X. H. F., Kim, J. Y., Kris, M. G., Ladanyi, M., Gerald, W. L. and Massague, J. (2009). WNT/TCF signaling though LEF1 and HOXB9 mediates lung adenocarcinoma metastasis. Cell. 138(1): 51-62.

Niesel, K., Schulz, M., Anthes, J., Alekseeva, T., Macas, J., Salamero-Boix, A., Möckl, A., Oberwahrenbrock, T., Lolies, M., Stein, S., Plate, K., Reiss, Y., Rödel, F. and Sevenich, L. (2021). The immune suppressive microenvironment affects efficacy of radio-immunotherapy in breast-to-brain metastasis. EMBO Mol Med. doi: 10.15252/emmm.202013412.

Noy, R. and Pollard, J. W. (2014). Tumor-associated macrophages: from mechanisms to therapy. Immunity. 41: $49-61$.

Olah, M., Biber, K., Vinet, J. and Boddeke, H. W. G. M. (2011). Microglia Phenotype Diversity. CNS Neurol Disord Drug targets. 10: 108 - 118.

Oliveros, J. C. (2007-2015). Venny. An interactive tool for comparing lists with Venn's diagrams.

Pasterkamp, R. J. and Giger, R. J. (2009). Semaphorin Function in Neural Plasticity and Disease. Curr Opin Neurobiol. 19(3): 263 - 274.

Plate, K. H., Scholz, A. and Dumont, D. J. (2012). Tumor angiogenesis and anti-angiogenic therapy in malignant gliomas revisited. Acta Neuropathol. 124: $763-775$.

Priego, N., Zhu, L., Monteiro, K., Mulders, M., Wasilewski, D., Bindeman, W., Doglio, L., Martinez, L., Martinez-Saez, E., Ramon y Cajal, S., Megias, D., Hernandez-Encinas, E., Blanco-Aparicio, C., Martinez, L., Zarzuela, E., Munoz, J., Fustero-Torre, C., Pineiro-Yanez, E., Hernandez-Lain, A., Bertero, L., Poli, V., Sanchez-Martinez, M., Menendez, J. A., Soffietti, R., Bosch-Barrera, J. and Valiente, M. (2018). STAT3 labels a subpopulation of reactive astrocytes required for brain metastasis. Nat Med. 24: 1024-1035.

Pyonteck, S. M., Akkari, L., Schuhmacher, A. J., Bowman, R. L., Sevenich, L., Quail, D. F., Olson, O. C., Quick, M. L., Huse, J. T., Teijeiro, V., Setty, M., Leslie, C. S., Oei, Y., Pedraza, A., Zhang, J., Brennan, C. W., Sutton, J. C., Holland, E. C., Daniel, D. and Joyce, J. A. (2013) CSF-1R inhibition alters macrophage polarization and blocks glioma progression. Nat Med. 19(10): 1264-1272.

Qiao, S., Qian, Y., Xu, G., Luo, Q. and Zhang, Z. (2019). Long-term characterization of activated microglia/macrophages facilitating the development of experimental brain metastasis through intravital microscopic imaging. $J$ Neuroinf. 16:4. doi: 10.1186/s12974-018-1389-9.

Quail, D. F., Bowman, R. L., Akkari, L., Quick, M. L., Schuhmacher, A. J., Huse, J. T., Holland, E. C., Sutton, J. C., and Joyce, J. A. (2016). The tumor microenvironment underlies acquired resistance to CSF-1R inhibition in gliomas. Science. 352 (6288): aad3018.

Quail, D. F. and Joyce, J. A. (2017). The Microenvironmental Landscape of Brain Tumors. Cancer Cell. 31(3): $326-341$.

R Core Team. (2014-2019). R: A language and environment for statistical computing. R Foundation for Statistical Computing, Vienna, Austria. URL. http://www.R-project.org/.

Rajan, W. D., Wojtas, B., Gielniewski, B., Gieryng, A., Zawadzka, M. and Kaminska, B. (2018). Dissecting functional phenotypes of microglia and macrophages in the rat brain after transient cerebral ischemia. Glia. 67: $232-245$.

Reis, E. S., Mastellos, D. C., Hajishengallis, G. and Lambris, J. D. (2019). New insights into the immune functions of complement. Nat Rev Immunol. 19: 503-516. 
Rubio-Perez, C., Planas-Rigol, E., Trincado, J. L., Bonfill-Teixidor, E., Arias, A., Marchese, D., Moutinho, C., Serna, G., Pedrosa, L., lurlaro, R., Martinez-Ricarte, F., Escudero, L., Cordero, E., Cicuendez, M., Ruiz, S., Parra, G., Nuciforo, P., Gonzalez, J., Pineda, E., Sahuquillo, J., Tabernero, J., Heyn, H. and Seoane, J. (2021). Immune cell profiling of the cerebrospinal fluid enables the characterization of the brain metastasis microenvironment. Nat Commun. 12: 1503.

Ruffell, B. and Coussens, L. M. (2015). Macrophages and Therapeutic Resistance in Cancer. Cancer Cell. 27: $462-472$.

Salamero-Boix, A., Schulz, M. and Sevenich, L. (2021). Activation of microglia and macrophages in neurodegenerative diseases. In: Towards Translating Biomarker Research of Neurodegenerative Diseases to Clinical Practice.

Springer - Neuromethods. in press.

Sankowski, R., Böttcher, C., Masuda, T., Geirsdottir, L., Sagar, Sindram, E., Seredenina, T:, Muhs, A., Scheiwe, C., Shah, M. J., Heiland, D. H., Schnell, O., Grün, D., Priller, J. and Prinz, M. (2019). Mapping microglia states in the human brain through the integration of high-dimensional techniques. Nat Neurosci. 22: $2098-2110$.

Schafflick, D., Xu, C. A., Hartlehnert, M., Cole, M., Schulte-Mecklenbeck, A., Lautwein, T., Wolbert, J., Heming, M., Meuth, S. G., Kuhlmann, T., Gross, C. C., Wiendl, H., Yosef, N. and Meyer zu Horste, G. (2020). Integrated single cell analysis of blood and cerebrospinal fluid leukocytes in multiple sclerosis. Nat Commun. 11: 247.

Schirmer, L., Velmeshev, D., Holmqvist, S., Kaufmann, M., Werneburg, S., Jung, D., Vistnes, S., Stockley, J. H., Young, A., Steindel, M., Tung, B., Goyal, N., Bhaduri, A., Mayer, S., Broder Engler, J., Bayraktar, O. A., Franklin, R. J. M., Haussler, M., Reynolds, R., Schafer, D. P., Friese, M. A., Shiow, L. R., Kriegstein, A. R. and Rowitch, D. H. (2019). Neuronal vulnerability and multilineage diversity in multiple sclerosis. Nature.

573: 75- 82.

Schroeder, T., Bittrich, P., Kuhne, J. F., Noebel, C., Leischner, H., Fiehler, J., Schroeder, J., Schoen, G. and Gellißen, S. (2020). Mapping distribution of brain metastases: does the primary tumor matter? J Neuro-Oncol. 147: 229 - 235.

Schulz, M., Salamero-Boix, A., Niesel, K., Alekseeva, T. and Sevenich, L. (2019). Microenvironmental Regulation of Tumor Progression and Therapeutic Response in Brain Metastasis. Front. Immunol. 10:1713. doi: 10.3389/fimmu.2019.01713.

Schulz, M., Michels, B., Niesel, K., Stein, S., Farin, H., Rödel, F. and Sevenich, L. (2020). Cellular and Molecular Changes of Brain Metastases-Associated Myeloid Cells during Disease Progression and Therapeutic Response. iScience. 23: 101178.

Scott, A. C., Dündar, F:, Zumbo, P., Chandran, S. S., Klebanoff, C. A., Shakiba, M., Trivedi, P., Menocal, L., Appleby, H., Camara, S., Zamarin, D., Walther, T., Snyder, A., Femia, M. R., Comen, E. A., Wen, H. Y., Hellmann, M. D., Anandasabapathy, N., Liu, Y., Altorki, N. K., Lauer, P., Levy, O., Glickman, M. S., Kaye, J., Betel, D., Philip, M. and Schietinger, A. (2019). TOX is a critical regulator of tumour-specific T cell differentiation. Nature. 571: $270-274$.

Seano, G., Nia, H. T., Emblem, K. E., Datta, M., Ren, J., Krishnan, S., Kloepper, J., Pinho, M. C., Ho, W. W., Ghosh, M., Askoxylakis, V., Ferraro, G. B., Riedemann, L., Gerstner, E. R., Batchelor, T. T., Wen, P. Y., Lin, N. U., Grodzinsky, A. J., Fukumura, D., Huang, P., Baish, J. W., Padera, T. P., Munn, L. L. and Jain, R. K. (2019). Solid stress in brain tumours causes neuronal loss and neurological dysfunction and can be reversed by lithium. Nat Biomed Eng. 3: $230-245$.

Seano, G. and Jain, R. K. (2020). Vessel co-option in glioblastoma: emerging insights and opportunities. Angiogenesis. 23: $9-16$.

Segarra, M., Aburto, M. R. and Acker-Palmer, A. (2021). Blood-Brain Barrier Dynamics to Maintain Brain Homeostasis. Trends Neurosci. doi: 10.1016/j.tins.2020.12.002. 
Sevenich, L., Bowman, R. L, Mason, S. D., Quail, D. F., Rapaport, F., Elie, B. T., Brogi, E., Brastianos, P. K., Hahn, W. C., Holsinger, L. J., Massague, J., Leslie, C. S. and Joyce, J. A. (2014). Analysis of tumour-and stroma-supplied proteolytic networks reveals a brain-metastasis-promoting role for cathepsin S. Nat Cell Biol. 16(9): 876888.

Sevenich, L. (2018). Brain-Resident Microglia and Blood-Borne Macrophages Orchestrate Central Nervous System Inflammation in Neurodegenerative Disorders and Brain Cancer. Front Immunol. 9: 697.

Sevenich, L. (2019). Turning "Cold" Into "Hot" Tumors - Opportunities and Challenges for Radio-Immunotherapy Against Primary and Metastatic Brain Cancers. Front Oncol. 9: 163.

Sharma, P., Hu-Lieskovan, S., Wargo, J. A. and Ribas, A. (2017). Primary, Adaptive, and Acquired Resistance to Cancer Immunotherapy. Cell. 168: 707 - 723.

Shi, W., Liao, Y., Willis, S. N., Taubenheim, N., Inouye, M., Tarlinton, D. M., Smyth, G. K., Hodgkin, P. D., Nutt, S. L. and Corcoran, L. M. (2015). Transcriptional profiling of mouse $B$ cell terminal differentiation defines a signature for antibody-secreting plasma cells. Nat Immunol. 16(6): 663 - 673.

Shih, D. J. H., Nayyar, N., Bihun, I., Dagogo-Jack, I., Gill, C. M., Aquilanti, E., Bertalan, M., Kaplan, A., D’Ándrea, M. R., Chukwueke, U., Ippen, F. M., AlvarezBreckenridge, C., Camarda, N. C., Lastrapes, M., McCabe, D., Kuter, B., Kaufmann, B., Strickland, M. R., Martinez-Gutierrez, J. C., Nagabhushan, D., De Sauvage, M., White, M. D., Castro, B. A., Hoang, K., Kaneb, A., Batchelor, E. D., Ha Paek, S., Park, S. H., Martinez-Lage, M., Berghoff, A. S., Merrill, P., Gerstner, E. R., Batchelor, T. T., Frosch, M. P., Frazier, R. P., Borger, D. R., lafrate, A. J., Johnson, B. E., Santagate, S., Preusser, M., Cahill, D. P., Carter, S. L. and Brastianos, P. K. (2020). Genomic characterization of human brain metastases identifies drivers of metastatic lung adenocarcinoma. Nat Genet. 52: $371-377$.

Slowikowski, K. (2020). ggrepel: Automatically Position Non-Overlapping Text Labels with 'ggplot2'. https://CRAN.R-project.org/package=ggrepel.

Soffietti, R., Abacioglu, U., Baumert, B., Combs, S. E., Kinhult, S., Kros, J. M., Marosi, C., Metellus, P., Radbruch, A., Villa Freixa, S. S., Brada, M., Carapella, C. M., Preusser, M., Le Rhun, E., Ruda, R., Tonn, J. C., Weber, D. C. and Weller, M. (2017). Diagnosis and treatment of brain metastases from solid tumors: guidelines from the European Association of Neuro-Oncology (EANO). Neuro-Oncol. 19(2): 162 - 174.

Sofroniew, M. V. (2009) Molecular dissection of reactive astrogliosis and glial scar formation. Trends Neurosci. 32(12): $638-647$.

Sofroniew, M. V. and Vinters, H. V. (2010). Astrocytes: biology and pathology. Acta Neuropathol. 119: $7-35$.

Song, E., Mao, T., Dong, H., Boisserand, L. S. B., Antila, S., Bosenberg, M., Alitalo, K., Thomas, J. L., and Iwasaki, A. (2020). VEGF-C-driven lymphatic drainage enables immunosurveillance of brain tumours. Nature. 577: 689 - 694.

Sperduto, P.W., Mesko, S., Li, J., Cagney, D., Aizer, A., Lin, N. U., Nesbit, E., Kruser, T. J., Chan, J., Braunstein, S., Lee, J., Kirkpatrick, J. P., Breen, W., Brown, P. D., Shi, D., Shih, H. A., Soliman, H., Sahgal, A., Shanley, R., Sperduto, W. A., Lou, E., Everett, A., Boggs, D. H., Masucci, L., Roberge, D., Remick, J., Plichta, K., Buatti, J. M., Jain, S., Gaspar, L. E., Wu, C.-C., Wang, T. J. C., Bryant, J., Chuong, M., An, Y., Chiang, V., Nakano, T., Aoyama, H. and Mehta, M. P. (2020). Survival in Patients With Brain Metastases: Summary Report on the Updated Diagnosis-Specific Graded Prognostic Assessment and Definition of the Eligibility Quotient. J Clin Oncol. 38(32): 1 - 12.

Sporn, M. B. (1996). The war on cancer. Lancet. 347: 1377-1381.

Stafford, J. H., Hirai, T., Deng, L., Chernikova, S. B., Urata, K., West, B. L. and Brown, J. M. (2016). Colony stimulating factor 1 receptor inhibition delays recurrence of glioblastoma after radiation by altering myeloid cell recruitment and polarization. Neuro-Oncol. 18(6): $797-806$. 
Stevens, A., Klöter, I. and Roggendorf, W. (1988). Inflammatory Infiltrates and Natural Killer Cell Presence in Human Brain Tumors. Cancer. 61: 738 - 743.

Stoletov, K., Strnadel, J., Zardouzian, E., Momiyama, M., Park, F. D., Kelber, J. A., Pizzo, D. P., Hoffman, R., VandenBerg, S. R. and Klemke, R. L. (2013). Role of connexins in metastatic breast cancer and melanoma brain colonization. J Cell Sci. 126: $904-913$.

Suh, J. H., Kotecha, R., Chao, S. T., Ahluwalia, M. S., Sahgal, A. and Chang, E. L. (2020). Current approaches to the management of brain metastases. Nat Rev Clin Oncol. 17: $279-299$.

Sundstrom, T., Daphu, I., Wendelbo, I., Hodneland, E., Lundervold, A., Immervoll, H., Skaftnesmo, K. O., Babic, M., Jendelova, P., Sykova, E., LundJohansen, M., Bjerkvig, W. and Thorsen. F. (2013). Automated Tracking of Nanoparticle-labeled Melanoma Cells Improves the Predictive Power of a Brain Metastasis Model. Cancer Res. 73: 2445 - 2456.

Tang, Y., Horikoshi, M. and Li, W. (2016). ggfortify: Unified Interface to Visualize Statistical Result of Popular R Packages. $R$ J. 8.2: 478-489.

Tenner, A. J., Stevens, B. and Woodruff, T. M. (2018). New tricks for an ancient system: Physiological and pathological roles of complement in the CNS. Mol Immunol. 102: 3 $-13$.

Tsao, M. N., Xu, W. and Sahgal, A. (2012a). A Meta-Analysis Evaluating Stereotactic Radiosurgery, Whole-Brain Radiotherapy, or Both for Patients Presenting with a Limited Number of Brain Metastases. Cancer. 118: 2486 - 2493.

Tsao, M. N., Xu, W., Wong, R. K. S., Lloyd, N., Laperriere, N., Sahgal, A., Rakovitch, E. and Chow, E. (2012b). Whole brain radiotherapy for the treatment of newly diagnosed multiple brain metastases. Cochrane Database Syst Rev. 4(6): 349 - 355.

Török, O., Schreiner, B., Schaffenrath, J., Tsai, H.-C., Maheshwari, U., Stifter, S. A., Welsh, C., Amorim, A., Sridhar, S., Utz, S. G., Mildenberger, W., Nassiri, S., Delorenzi, M., Aguzzi, A., Han, M. H., Greter, M., Becher, B. and Keller, A. (2021). Pericytes regulate vascular immune homeostasis in the CNS. Proc Natl Acad Sci. 118(10): e2016587118.

Utz, S. G., See, P., Mildenberger, W., Thion, M. S., Silvin, A., Lutz, M., Ingelfinger, F., Rayan, N. A., Lelios, I., Buttgereit, A., Asano, K., Prabhaker, S., Garel, S., Becher, B., Ginhoux, F. and Greter, M. (2020). Early Fate Defines Microglia and Nonparenchymal Brain Macrophage Development. Cell. 181: 1-17.

Valiente, M., Obenauf, A. C., Jin, X., Chen, Q., Zhang, X. H.-F., Lee, D. J., Chaft, J. E., Kris, M. G., Huse, J. T., Brogi, E. and Massague, J. (2014). Serpins Promote Cancer Cell Survival and Vascular Co-Option in Brain Metastasis. Cell. 156: $1002-106$.

Valiente, M., Ahluwalia, M. S., Boire, A., Brastianos, P. K., Goldberg, S. B., Lee, E. Q., Le Rhun, E., Preusser, M., Winkler, F. and Soffietti, R. (2018). The Evolving Landscape of Brain Metastasis. Trends Cancer. 4(3): 176 - 196.

van Vulpen, M., Kal, H. B., Taphoorn, M. J. B. and El Sharouni, S. Y. (2002) Changes in blood-brain barrier permeability induced by radiotherapy: Implications for timing of chemotherapy? (Review). Oncol Rep. 9: 683 - 688.

Venkataramani, V., Tanev, D. I., Strahle, C., Studier-Fischer, A., Frankhauser, L., Kessler, T., Körber, C., Kardorff, M., Ratliff, M., Xie, R., Horstmann, H., Messer, M., Paik, S. P., Knabbe, J., Sahm, F., Kurz, F. T., Acikgöz, A. A., Hermannsdörfer, F., Agarwal, A., Bergles, D. E., Chalmers, A., Miletic, H., Turcan, S., Mawrin, C., Hänggi, D., Liu, H.-K., Wick, W., Winkler, F. and Kuner, T. (2019). Glutamatergic synaptic input to glioma cells drives brain tumour progression. Nature. 573: $532-538$.

Venkatesh, H. S., Morishita, W., Geraghty, A. C., Silverbush, D., Gillespie, S. M., Arzt, M., Tam, L. T., Espenel, C., Ponnuswami, A., Ni, L., Woo, P. J., Taylor, K. R., Agarwal, A., Regev, A., Brang D., Vogel, H., Hervey-Jumper, S., Bergles, D. E.., Suva, M. L., Malenka, R. C. and Monje, M. (2019). Electrical and synaptic integration of glioma into neural circuits. Nature. 573: $539-545$. 
Warnes, G. R., Bolker, B., Bonebakker, L., Gentleman, R., Huber, W., Liaw, A., Lumley, T., Maechler, M., Magnusson, A., Moeller, S., Schwartz, M. and Venables, B. (2020). gplots: Various $\mathrm{R}$ Programming Tools for Plotting Data.https://CRAN.Rproject.org/package=gplots.

Wasilewski, D., Priego, N., Fustero-Torre, C. and Valiente, M. (2017). Reactive Astrocytes in Brain Metastasis. Front Oncol. 7: 298. doi: 103389/fonc.2017.00298.

Wickham, H. (2007). Reshaping Data with the reshape Package. J Stat Software. 21(12): $1-20$.

Wickham, H. (2016). Ggplot2: Elegant Graphics for Data Analysis. SpringerVerlag, New York.

Wickham, H., Francois, R., Henry, L. and Müller, K. (2017). dplyr: A Grammar of Data Manipulation. https://CRAN.R-project.org/package=dplyr.

Wculek, S. K., Cueto, F. J., Mujal, A. M., Melero, I., Krummel, M. F. and Sancho, D. (2019). Dendritic cells in cancer immunology and immunotherapy. Nat Rev Immunol. 20: $7-24$

Yu, G., Wang, L.-G., Han, Y. and He, Q.-Y. (2012). clusterProfiler: an R Package for Comparing Biological Themes Among Gene Clusters. OMICS. 16(5): 284 - 287.

Yu, G. (2020). enrichplot: Visualization of Functional Enrichment Result. https://yulab-smu.top/biomedical-knowledge-mining-book/.

Yushkevich, P. A., Pashinskiy, A., Oguz, I., Mohan, S., Schmitt, J. E., Stein, J M., Zukic, D., Vicory, J., McCornick, M., Yushkevich, N., Schwartz, N., Gao, Y. and Gerig, G. (2019). User-Guided Segmentation of Multi-modality Medical Imaging Datasets with ITK-SNAP. Neuroinformatics. 17: 83 -102.

Zeiner, P. S., Zinke, J., Kowalewski, D. J., Bernatz, S., Tichy, J., Ronellenfitsch, M. W., Thorsen, F., Berger, A., Forster, M. T., Muller, A., Steinbach, J. P., Beschorner, R., Wischhusen, J., Kvasnicka, H. M., Plate, K. H., Stefanovic, S., Weide, B., Mittelbronn, M. and Harter, P. N. (2018). CD74 regulates complexity of tumor cell HLA class II peptidome in brain metastasis and is a positive prognostic marker for patient survival. Acta Neuropathol Commun. 6: 18.

Zeng, Q., Michael, I. P., Zhang, P., Saghafinia, S., Knott, G., Jiao, W., McCabe, B. D., Galvan, J. A., Robinson, H. P. C., Zlobec, I., Ciriello, G. and Hanahan, D. (2019). Synaptic proximity enables NMDAR signalling to promote brain metastasis. Nature. 573: 526-531.

Zhou, Y., Zhou, B., Pache, L., Chang, M., Khodabakhshi, A. H., Tanaseichuk, O., Benner, C. and Chanda, S. K. (2019). Metascape provides a biologist-oriented resource for the analysis of systems-level datasets. Nat Commun. 10: 1523.

Zhou, Y., Song, W. M., Andhey, P. S., Swain, A., Levy, T., Miller, K. R., Poliani, P. L., Cominelli, M., Grover, S., Gilfillan, S., Cella, M., Ulland, T. K., Zaitsev, K., Miyashita, A., Ikeuchi, T., Sainouchi, M., Kakita, A., Bennett, D. A., Schneider, J. A., Nichols, M. R., Beausoleil, S. A., Ulrich, J. D., Holtzman, D. M., Artyomov, M. N. and Colonna, M. (2020). Human and mouse single-nucleus transcriptomics reveal TREM2-dependent and TREM2-independent cellular responses in Alzheimer's disease. Nat Med. 26: $131-142$. 


\section{List of Publications and Contributions}

\section{Publications}

\section{published}

Schulz, M., Michels, B., Niesel, K., Stein, S., Farin, H., Rödel, F. and Sevenich, L. (2020). Cellular and Molecular Changes of Brain Metastases-Associated Myeloid Cells during Disease Progression and Therapeutic Response. iScience 23, 101178.

research article

Schulz, M.* , Salamero-Boix, A. ${ }^{*}$, Niesel, K. ${ }^{*}$ Alekseeva, T. ${ }^{*}$ and Sevenich, L. (2019). Microenvironmental Regulation of Tumor Progression and Therapeutic Response in Brain Metastasis. Front. Immunol. 10:1713. doi: 10.3389/fimmu.2019.01713.

*shared co-first author, review article

Salamero-Boix, A. ${ }^{*}$, Schulz, M.*, and Sevenich, L. (2021). Activation of microglia and macrophages in neurodegenerative diseases. In: Towards Translating Biomarker Research of Neurodegenerative Diseases to Clinical Practice. Springer Neuromethods.

*shared co-first author, book chapter, in press

in preparation

Schulz, M. and Sevenich, L. A protocol for live cell imaging of an artificial brain metastases microenvironment.

research method article

Schulz, M. and Sevenich, L. TAMs in Brain Metastasis: Molecular signatures in mouse and man. Front Oncol.

review article 


\section{co-Author Contributions}

\section{published}

Chae, W. H., Niesel, K., Schulz, M., Klemm, F., Joyce, J. A., Prümmer, M., Brill, B., Bergs, J., Rödel, F., Pilatus, U. and Sevenich, L. (2019). Evaluating Magnetic Resonance Spectroscopy as a Tool for Monitoring Therapeutic Response of Whole Brain Radiotherapy in a Mouse Model for Breast-to-Brain Metastasis. Front. Oncol. 9:1324. doi: 10.3389/fonc.2019.01324.

research article

Niesel, K., Schulz, M., Anthes, J., Alekseeva, T., Macas, J., Salamero-Boix, A., Möckl, A., Oberwahrenbrock, T., Lolies, M., Stein, S., Plate, K. H., Reiss, Y., Rödel, F. and Sevenich, L. (2021). The immune suppressive tumor microenvironment affects efficacy of radio-immunotherapy in breast-to-brain metastasis. EMBO Mol Med. doi: 10.15252/emmm.202013412.

research article

\section{submitted/in revision}

Klemm, F., Moeckl*, A., Salamero-Boix*, A., Alekseeva, T., Schaeffer, A., Schulz, M., Niesel, K., Maas, R. R., Groth, M., Elie, B. T., Bowman, R. L., Hegi, M. E., Daniel, R. T., Zeiner, P. S., Zinke, J., Harter, P. N., Plate, K. H., Joyce, J. A. and Sevenich, L. (2021). Compensatory CSF-2-driven macrophage activation promotes adaptive resistance to CSF1R inhibition in breast-to-brain metastasis. Nat Cancer. in revision.

research article

\section{in preparation}

Rosigkeit, S., Röder, J., Kruchem, M., Thies, D., Siegl, D., Kreft, A., Schulz, M., Pickert, G., Hayduk, N., Heck, R., Kaps, L., Foerster, F., Hähnel, P., Kindler, T., Hartwig, U., Guerra, C., Barbacid, M., Wirth, D., Sevenich, L., Schuppan, D. and Bockamp, E. A reporter gene-equipped murine lung cancer model resembling human non-small cell lung cancer.

research article 


\section{Conference Contributions}

>>Parts of this thesis have been published as oral or poster presentations. $<<$

\section{Oral contribution}

"UCT Science Day's", Frankfurt, Germany 2019

"The Myeloid Immune Cell Landscape in Lung-to-Brain Metastasis"

Schulz, M., Niesel, K., Michels, B., Stein, S., Farin, H., Rödel, F. and Sevenich, L.

\section{Poster contribution}

"UCT Science Day's", Frankfurt, Germany

- 2016

"Targeting Cancer-Associated Inflammation in Brain Metastasis"

Schulz, M., Niesel, K., Stein, S., Rödel, F. and Sevenich, L.

- 2017

"Lymphocytes Contribute to a Highly Dynamic

Microenvironment in Breast-to-Brain Metastasis"

Schulz, M., Chae, W. H., Niesel, K., Stein, S., Rödel, F. and Sevenich, L.

"Frankfurt Cancer Conference", Frankfurt, Germany

- 2018

"Effects of lonizing Irradiation on Brain Metastasis-Associated

Inflammation and its Implication for Immuno-Therapy"

Schulz $^{*}$, M., Niesel ${ }^{*}$, K., Salamero-Boix, A., Chae, W. H., Alekseeva, T., Michels, B., Farin, H., Rödel, F., Harter, P., Plate, K.-H. and Sevenich, L.

"EMBO Workshop Microglia 2018", Heidelberg, Germany

- 2018

"Irradiated Murine Microglia Upregulate Neuro-Inflammation and Inflammasome-Related Pathways in a Xenograft Lung-to-Brain Metastasis Model"

Schulz, M., Michels, B., Stein, S., Rödel, F. and Sevenich, L.

"EACR-AACR Basic and Translational Research Conference - TUMOR MICROENVIRONMENT", Lisbon, Portugal

- 2020

"Cellular and Molecular Changes of Brain Metastases-Associated Myeloid Cells during Disease Progression and Therapeutic Response"

Schulz, M., Michels, B., Niesel, K., Stein, S., Farin, H., Rödel, F. and Sevenich, L. 